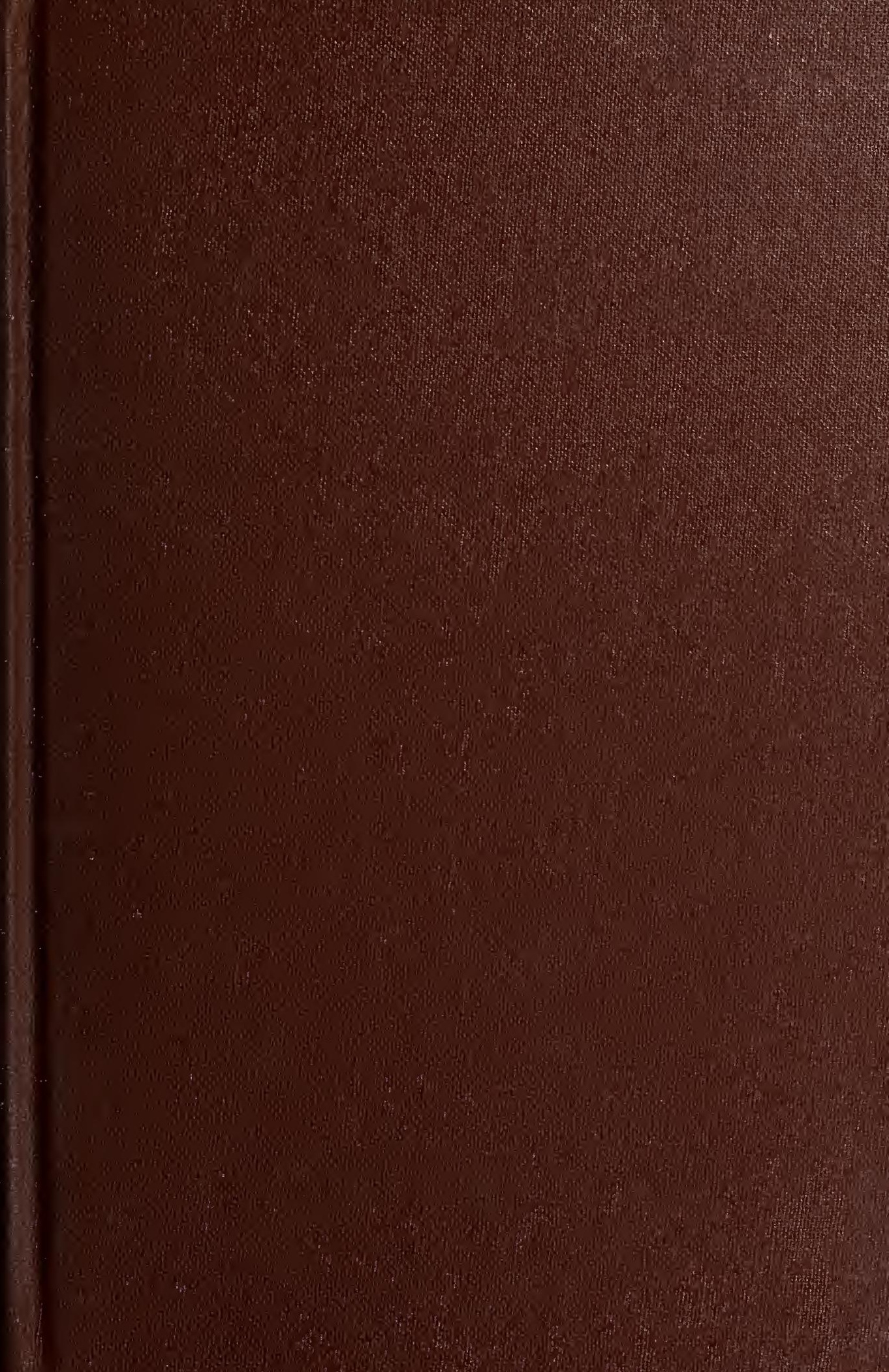


OP

$\mathrm{Ab}$

c

BRI

Natural History Miuseum Library

- IIIIIIIIIIIIIIII

000159382 




Bele. 



\section{CATALOGUE}

OF THE

\section{$\mathrm{BOOKS}$}

IN THE

\section{DEPARTMENT OF ZOOLOGY}

(BRITISH IIUSEUM, NATURAL HISTORY).

$$
\text { s sh }
$$

THIRD EDITION.

PRINTED BY ORDER OF THE TRUSTEES.

1884 . 


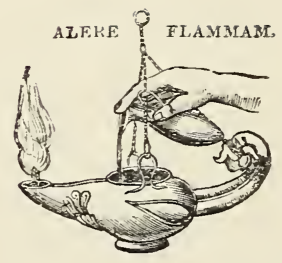

PRINTED RY TAYLOR AND FRANCIS,

RED LIOX COURT, FLEET STRFET. 


\section{(TO FIRST EDITION.)}

The Library of the Zoological Department consists, 1st, of works acquired from the small annual grants or by means of exchange for the publications of the Department; 2nd, of books received as donations; and, 3rd, of the purely zoological works which were purchased out of the extraordinary grant made in the last financial year (1879-80). All these works are enumerated in the present List.

Separate copies of memoirs and papers published in periodicals or other mixed publications have only been admitted under distinct titles when they have been brought. into the book-market, many Academies and Societies publishing and selling their memoirs separately; but the separate copies of papers, of which a small number has been printed for the authors for private distribution, are not included.

The Zoological Library is especially poor in old works ; it contains only 70 of a date earlier than the year 1801 .

As this List has been printed chiefly with the view of serving as a temporary guide in making additions to the Zoological Library, and of preventing the acquisition of duplicates for the General Natural History Library, no bibliographical completeness of the titles has been attempted; on the contrary, the titles have been as much abridged as seemed to be consistent with the recognition of the works catalogued.

British Museum,

ALBERT GÜNTHER.

Nov. 26, 1880.

\section{(TO SECOND EDITION.)}

Is the present second issue of the 'Catalogue of Books in the Department of Zoology, the works acquired between November 26, 1850, and May 1, 1882, have been inserted. It contains 2638 titles as against 1700 of the first issue, beside 92 titles of works which belonged to the Zoological Department and have been transferred to the General Library: these are enumerated at the end of this Catalogue.

British Museum,

ALBERT GÜNTHER.

May 1882. 
Since the issue of the second edition of this Catalogue the number of works in the Department has been nearly doubled. This part of the Natural History Library contains now 5309 works, represented by 8586 volumes. Beside the main titles of these works, 823 cross-references have been inserted in the present edition.

Care having been taken to collect in the first instance those works which are most frequently required for the arrangement and study of the collections, the Departmental Library, combined with the General Library, fairly fulfils its primary object. But it is still far from being complete, and, as many of the works which are out of print, are very scarce, some difficulty is experienced in obtaining suitable copies. It is hoped, however, that in the course of two or three years, the library will have sufficiently advanced towards completeness to allow of the Alphabetical List, which is the most convenient during the progress of the formation of a Library, being superseded by a systematically-arranged Catalogue.

This third edition has been prepared by Mr. J. E. Harting, F.L.S.

\section{ALBERT GÜNTHER.}

Department of Zoology,

March 17, 1884. 


\title{
DEPARTMENT OF ZOOLOGY.
}

\author{
CATA LOG UE \\ OF \\ L I B R A R Y.
}

A.

ABBOT (John). Drawings of the Insects of Georgia, in America. [Original MS.] 17 vols. $4^{\circ}$. 1792-1804.

ABBOT (J.) and SMITH (J.E.). The Natural History of the rarer Lepidopterous Insects of Georgia.

2 vols. fol. London, 1797.

ABD-EL-KADER (The Emir). See Daduas (E.).

ABEL (J. C. A. M.). Die Conchylien in dem NaturalienCabinette des Bischofs von Konstanz.

12. Bregenz, 1787.

ABICH (H.). Vergleichende chemische Untersuchungen der Wasser des Caspischen Meeres, Urmia- und Van-See's.

$4^{\circ}$. St. Petersburg, 1856.

ACHILle (G.). See Periodicals : Milan.

ADAMS (A.). Travels of a Naturalist in Japan and Manchuria. $\quad 8^{\circ}$. London, 1870.

ADAMS (A.). See 'Samarang.'

ADAMS (C. B.). Catalogue of the Genera and Species of recent Shells.

8. . Middleburg, 1847.

ADAMS (C. B.). Monograph of Stoastoma.

4\%. Amherst, 1849. 
ADAMS (C. B.). Monograph of Vitinella.

4\%. Amherst, 1850 .

ADAMS (C. B.). Contributions to Conchology.

$8^{\circ}$. New York, 1849-52.

ADAMS (C. B.), Catalogue of Shells collected at Panama.

$4^{\circ}$. New York, 1852.

ADAMS (H. and A.). The Genera of recent Mollusca.

3 vols. $8^{\circ} . \quad$ London, 1858.

ADAMS (H. G.). Our Feathered Families. The Birds of Song.

$8^{\circ}$. London, n. d.

ADAMS (H. G.). Our Feathered Families. The Birds of Prey.

8. London, n. d.

ADAMS (H. G.). Our Feathered Families. Game and Water Birds.

8. London, n. d.

ADAMS (H. G.). Beautiful Butterflies.

$8^{\circ}$. London, 1871.

ADAMS (W.H. D.). The Mysteries of the Ocean. From the French of Arthur Mangin. New edition.

$8^{\circ}$. London, 1870.

ADAMS (W. M.). See Fisherins : London.

ADAMSON (C. M.). Another book of Scraps, principally relating to Natural History.

obl. $4^{\circ}$. London, 1882 .

ADANSON (M.). Histoire Naturelle du Sénégal (Coquitlages).

$4^{\circ}$. Paris, 1757.

L'ADMIRAL (J.). Naauwkeurige waarnemingen omtrent de Veranderingen van veele Insekten of gekorvene diertjes.

$4^{\circ}$. Amsterdam, 1774.

AEBY (C.). Eine neue Methode zur Bestimmung der Schädelform von Menschen und Säugethieren.

$4^{\circ}$. Braunschweig, 1862. 
AEBY (C.). Die Schädelformen des Menschen und der Affen.

AEBY (C.). Der Bronchialbaum der Säugethiere und des Menschen.

8. Leipzig, 1880.

ELIANUS. De Natura Animalium.

$4^{\circ} . \quad$ Londini, 1744.

(Ed. Gronovius.) $4^{\circ}$. Basilea, 1774.

(Ed. Schneider.) $8^{\circ}$. Lipsice, 1784.

AGASSIZ (A.). Embryology of the Starfish.

$4^{\circ}$. Cambridge, Mass., 1864.

See Musedm: Harvard College. Memoirs.

AGASSIZ (A.). North-American Acalephce.

$4^{\circ}$. Cambridge, Mass., 1865.

See Museum : Harvard Collegr. Memoirs.

AGASSIZ (A.). Revision of the Echini.

2 vols. $4^{\circ}$. Cambridge, Mass., 1872-73.

See Musedm: Harvard College. Memoirs.

AGASSIZ (A.). Embryology of the Ctenophorce.

$4^{\circ}$. Cambridge, Mass., 1874.

See Museum : Harvard College. Memoirs.

AGASSIZ (A.) and POURTALES (L. F. de). Zoological Results of the Hassler Expedition. I. Echini, Crinoids, and Corals.

$4^{\circ}$. Cambridge, Mass., 1874.

See Museum : Harvard College. Memoirs.

AGASSIZ (A.). North-American Starfishes.

$4^{\circ}$. Cambridge, Mass., 1877.

See Musedu : Harvard College. Memoirs.

AGASSIZ (A.). Embryological Monographs. II. Echinodermata. $\quad 4^{\circ}$. Cambiidge, Mass., 1883.

See Musedu : Harvard College. "Memoirs. 
AGASSIZ (A.). Exploration of the Surface Fauna of the Gulf-Stream. Porpitidce and Velellidae.

$4^{\circ}$. Cambridge, Mass., 1883.

See Musedm : Harvard College. Memoirs.

AGASSIZ (A.). See 'Challenger.'

AGASSIZ (L.). Recherches sur les Poissons fossiles.

Text, 5 vols. in $2.4^{\circ}$.

Atlas, 5 vols. in 2. obl. fol. Paris, 1833-43.

AGASSIZ (L.). Rapport sur les Poissons fossiles déconverts en Angleterre.

8․ Neuchatel, 1835.

AGASSTZ (L.). Monographies d'Échinodermes vivants et fossiles.

$4^{\circ}$. Neuchátel, 1838.

AGASSIZ (L.). Mémoire sur les moules de Mollusques vivants et fossiles.

4. Neuchâtel, 1839.

AGASSIZ (L.). Catalogus Echinodermatum fossilium Musei Neocomensis. $\quad 4^{\circ}$. Néocomi Helveticorum, 1840.

AGASSIZ (L.). Histoire naturelle des Poissons d'eau douce de l'Europe centrale.

Texte, $4^{\circ}$. Planches, obl. fol. Neuchátel, 1839-42.

AGASSIZ (L.). Monographie des Poissons fossiles du vieux grès rouge ou système Déronien (Old Red Sandstone) des îles Britanniques et de Russie.

Texte, $4^{\circ}$. Atlas, obl. fol. Neuchâtel, 1844-45.

AGASSIZ (L.). Nomenclator Zoologicus.

4 vols. $4^{\circ}$. Soloduri, 1842-46.

AGASSIZ (L.). Nomenclatoris Zoologici Index Universalis.

$8^{\circ}$. Soloduri, 1848.

AGASSIZ (L.). Bibliographia Zoologiæ et Geologiæ. Ed. H. E. Strickland.

4 vols. $8^{\circ}$. $1848-54$.

See RAY Society.

AGASSIZ (L.). Twelve Lectures on Comparative Embryology.

$8^{\circ}$. Boston, 1849. 
AGASSIZ (L.). The Classification of Insects from embryological data.

$4^{\circ}$. Washington, 1850.

AGASSIZ (L.). Die Zoologie.

2 vols. in $1.8^{\circ}$. Stuttgart, 1855.

AGASSIZ (L.). Contributions to the Natural History of the United States of America. 4 vols. $4^{\circ}$. Boston, 1857.

AGASSIZ (L.). An Essay on Classification.

8. $\quad$ London, 1859

AGASSIZ (L.). The Structure of Animal Life.

8. London, 1866.

AGASSIZ (L.). Pisces Celebes.

fol. London, 1874 .

AGASSIZ (L.). Report on the Florida Reefs.

$4^{\circ}$. Cambridge, Mass., 1880.

See Museum: Harvard College. Memoirs.

AGASSIZ (L.). Seo Spix (J. B. de) et Agassiz (L.).

AGNELY (Dr.), LALLEMANT (C.), et DARRU (A.). Le Criquet Pèlerin (Acridium peregrinum). Sauterelle.

8. Alger et Paris, 1866.

AHLGREN (J.). See Dahrbom (G.).

AIGNER (J.). Salzburg's Fische. $\quad 8^{\circ}$. Salzburg, 1859. See Periodicals: Salzburg.

ALBERS (J. A.). Icones ad illustrandam Anatomen Comparatam. fol. Lipsice, 1818-22.

ALBERS (J. C.). Die Heliceen.

1st edition. $8^{\circ}$. Berlin, 1850. 2nd edition. $8^{\circ}$. Leipzig, 1860.

ALBERS (J. C.). Malacographia Madeirensis.

$4^{\circ}$. Berolini, 1854.

ALBERTUS MagnUS. See Friderici IT. 
ALBIN (E.). 'A Natural History of Birds.

3 vols. $4^{\circ}$. London, 1731-1738.

ALBIN (E.). A Natural History of English Insects.

$4^{\circ}$. London, 1720.

ALBIN (E.). Insectorum Angliæ Naturalis Historia.

$4^{\circ}$. Londini, 1731.

ALBIN (E.). A Natural History of Spiders, and other curious Insects.

$4^{\circ}$. London, 1736.

ALDER (J.). A Catalogue of the Land and Freshwater Testaceous Mollusca. With Supplement.

$4^{\circ}$. Newcastle, 1830.

ALDER (J.). Catalogue of the Mollusca of Northumberland and Durham.

8. . London, 1848.

ALDER (J.). Catalogue of the Zoophytes of Northumberland and Durham.

8. Newcastle-upon-Tyne, 1857. Supplement. $8^{\circ} .1860$.

ALDER (J.) and HANCOCK (A.). A Monograph of the British Nudibranchiate Mollusca. fol. London, 1845-55. See RAY SocIETY.

ALDROVANDUS (U.). Opera omnia.

13 vols. fol. Bononicue, 1623-1642.

ALDROVANDUS (U.). De Quadrupedibus digitatis viviparis (Libri tres) et de Quadrupedibus digitatis oviparis (Libri duo).

4\%. Bononice, 1637.

ALDROVANDUS (U.). Ornithologiæ, hoc est de avibus Historiæ Libri XII. fol. Francofurti, 1610.

'ALERT.' Cruise of H.M.S. 'Alert' in Patagonian, Polynesian and Muscarene Waters, 1878-82. By R. W. Coppinger.

$4^{\circ}$. London, 1883.

ALESSANDRI (I.). See Scattaglia (P.).

A LEXANDER (J. E.). Salmon-Fishing in Canada by a Resident. Edited by Col. Sir J. E. A. $8^{\circ}$. London, 1860. 
ALIX (E.). Essai sur l'Appareil Locomoteur des Oiseaux. 8. Paris, 1874.

ALIX (E.). See Gratiolet (L. P.).

ALLEN (C. A.). Fauna Lusitanica. [Annulosa.] 8. Porto d'Outubro, 1857.

ALLEN (H.). The Bats of North America.

$8^{\circ}$. Washington, 1864.

See Suithonian Miscell. Collection.

ILLEN (J. A.). The Mammals of Massachusetts.

See Museum : Harvard College. Bulletin.

8. Cambridge, 1869.

ALLEN (J. A.). On the Eared Seals, Otariidce.

80. Cambridge, 1870.

See Museum: Harvard College. Bulletin.

ALIEN (J. A.). An Ornithological Reconnoissance of Kansas, Colorado, Wyoming, and Utah.

See Museum : Harvard College. Bulletin.

$8^{\circ}$. Cambriclge, 1872.

ALLEN (J. A.). The American Bisons : living and extinct. $4^{\circ}$. Cambridge, 1876.

See Museum : Harvard College. Memoirs.

ALLEN (J. A.). A History of North-American Pinnipeds : a Monograph of the Walruses and Seals of North America. $8^{\circ}$. Washington, 1880.

See Surveys and Explorations: U. S., Washington.

ALLEN (J. A.). See Coues (E.) and Allen (J. A.).

allen (J. A.). See Museum : Harvard College.

ALLMAN (G. J.). A Monograph of the Freshwater Polyzoa. fol. London, 1856.

See RAY SocIETr.

ALLMAN (G. J.). A Monograph of the Gymnoblastic or Tubularian Hydroids.

fol. London, 1871-72.

See Ray Societr. 
ALLMAN (G. J.). Report on the Hydroida collected during the Exploration of the Gulf Stream. By L. F. de Pourtales.

See Mcsfem : Harvard College. Memoirs.

$4^{\circ}$. Cambridge, 1877.

ALLMAN (G. J.). See 'Challenger.'

ALSTON (E. R.). See BELL (T.).

ALston (E. R.). See Biologia Centrali-Anericana.

ALTENA (H. L. van). Species indigenæ Reptilium ex ordine Batrachiorum, \&c.

4. Lugduni Batavorum, 1829.

ALTON (E. D'). See Pander (C. H.).

ALTUM (B.). Der Togel und sein Leben geschildert.

8․ Münster, 1875.

ALTUM (B.). Die Geweihbildung bei Rothhirsch, Rehbock, Damhirsch : Ein Beitrag zur Jagdzoologie.

8. Berlin, 1874.

ALTUMI (B.). Die Geweihbildung des Elchhirsches.

8․ Berlin, 1875.

ALTUM (B.). Unsere Spechte und ihre forstliche Bedeutung.

80. Berlin, 1878.

ALTUM (B.). Forstzoologie.

3 vols. $8^{\circ}$. Bertin, 1876-82.

AMOREUX (M.). Notice des Insectes de la France.

8. Paris, 1789.

AMYOT (C. J. B.). Entomologie Française. Rhynchotes.

8. Paris, 1848.

AMYOT (C. J. B.) et SERVILLE (A.). Histoire naturelle des Insectes (Hémiptères).

so. Paris, 1843. 
ANDERSON (J.). The Organization and Progress of the Anderson School of Natural History at Penikese Island.

$8^{\circ}$. Cambridge, 1874.

ANDERSON (J.). Anatomical and Zoological Researches. 2 vols. $4^{\circ}$. London, 1878.

Seo Surveys and Explorations: Yunnan.

ANDERSON (J.). Catalogue of Mammalia in the Indian Museum. 80. Calcutta, 1881.

See Musedm (Calcutta).

ANDERSSON (C. J.). A Journey to Lake 'Ngami. 12․ Cape Town, 1854.

ANDERSSON (C. J.). Lake'Ngami. $\quad 8^{\circ}$. London, 1856.

ANDERSSON (C. J.). The Okavango River.

8. London, 1861.

ANDERSSON (C. J.). The Birds of Damaraland. Edited by John Henry Gurner.

8․ London, 1872.

ANDERSSON (C. J.). The Lion and the Elephant. Edited by L. LLoyd.

8. . London, 1873.

ANDERSSON (C. J.). Notes of Travel in South Africa. Edited by L. LLoYd.

8. London, 1875.

ANDRÉ (E.). Species des Hyménoptères d'Europe et d'Algérie.

8.. Paris, $1879-83$. [In progress.]

ANDRÉ (E.). La Structure et la Biologie des Insectes ; particulièrement des Hyménoptères. $\quad 8^{\circ}$. Beaune, 1882.

ANDRÉ (E.). See Brongmiart' (C. J. E.).

ANGAS (G. F.). South Australia Illustrated.

fol. London, 1847.

ANGAS (G. F.). The Kafirs illustrated in a series of Drawings.

fol. London, n. d. 
ANGELI (B.). De admirabili Viperæ natura, et de mirificis ejusdem facultatibus Liber. $8^{\circ}$. Noribergce, 1603.

ANIMALS : Memoirs for a Natural History of Animals. Published by the Royal Society.

$4^{\circ}$. London, 1701.

ANSELL (A. W.). See Fisheries : London.

ANTELME (A.). Histoire Naturelle des Insectes et.des Mollusques, sous la Direction de I. Geoffroy St.-Hilaire. 2 vols. $12^{\circ}$. Paris, 1841.

ANTINORI (O.). Catalogo descrittivo di una Collezione di Uccelli d' Affrica.

8. Milano, 1864.

APOSTOLIDES (N. C.). La pêche en Grèce.

8. Athènes, 1883.

ARADAS (A.) and BENOIT (L.). Conchigliologia Vivente Marina della Sicilia. Parts I.-III. $4^{\circ}$. Catania, 1870.

ARAGONA (A.). De quibusdam Coleopteris Italiæ novis aut rarioribus Tentamen.

8. Ticini Regii, n. d.

ARCAS (L. P.). Insectos nuevos de la Fauna Española.

Parts I.-III. 8. $\quad$ Madrid, 1865.

ARCTIC MANUAL.

8. London, 1875.

ARENDT (E.). De capitis ossei Esocis lucii structura singulari. $\quad 4^{\circ}$. Regiomonti, 1822.

ARGYLL (G. D. Campbell, Duke of). Primeral Man. 8. London, 1869.

ARISTOTELES. De Animalium partibus \&c.

2 vols. $4^{\circ} . \quad$ Francofurti, 1585-87.

ARISTOTELES. De Animalibus Historiæ.

(Ed. SchNeIder.) 4 vols. $8^{\circ}$. Lipsice, 1811.

ARISTOTELES. Aristoteles Thierkunde kritischberichtigter Text mit Deutscher Uebersetzung ron H. AठBBERT und F. Wimmer. 2 vols. $8^{\circ}$. Leipzig, 1868. 
ARISTOTELES. Histoire des Animaux d'Aristote, traduite en Français par J. BarthéLeuy St.-Hilaire.

3 vols. $8^{\circ}$. Paris, 1883.

ARISTOTELES. See Köhler (H. J.), Langkavel (B.), Philebert (H.), Sundevall (C. J.), Wiegmann (A. F. A.).

ARLONG (S.). See Chaudeau (A.).

ARSAKY (A.). Commentatio de Piscium cerebro et medulla spinali.

$4^{\circ}$. Lipsice, 1836.

ARTAUD (S.). See Blumenbach (J. F.).

ARTEDI (P.). Ichthyologia. $\quad 8^{\circ} . \quad$ Lugd. Batav., 1738.

ARTEDI (P.). Bibliotheca Ichthyologica (ed. J. J. WALBAOM).

2 vols. $8^{\circ}$. Grypeswaldice, 1788-89

ASMUS (I.). See Fisherres: BerLin. Internationale Fischerei-Ausstellung.

ASMUSS (H. M.). Monstrositates Coleopterorum.

$8^{\circ}$. Riga et Dorpati, 1835.

ATKINSON (J.). A Compendium of the Ornithology of Great Britain.

8. London, 1820.

ATKinson (W. S.). See Hewrtson (W. C.), Moore (F.), and Grote (A.).

AUBÉ (C.). See Dejean (Comte).

AUBERT (H.) and WIMMER (F.) See Aristoteles.

AUDEBERT (J. B.). Histoire Naturelle des Singes et des Makis.

fol. Paris. 1800.

AUDEBERT (J. B.) et VIEILLOT (L, P.). Histoire naturelle et générale des Grimpereaux et des Oiseaux de Paradis.

$4^{\circ}$. Paris, 1802. 
AUDEBERT (J. B.) et VIEILLOT (L. P.). Histoire naturelle et générale des Colibris, Oiseaux-Mouches, Jacamars et Promérops.

$4^{\circ} . \quad$ Paris, 1802.

AUDINET-SERVILLE (J. G.). Histoire Naturelle des Insectes Orthoptères.

8. Paris, 1839.

aUdinet-SERVILLE (J. G.). See Fadne Française.

AUDOUIN (J.'V.). Prodrome d'une Histoire naturelle chimique, pharmaceutique, et médicale des Cantharides.

$4^{\circ}$. Paris, 1826.

AUDOUIN (J. V.) et MILNE EDWARDS (H.). Recherches pour servir à l'histoire naturelle du littoral de la France.

2 vols. $8^{\circ}$. Paris, 1832-34.

AUDOUIN (J. V.). Recherches.... sur la Maladie contagieuse qui attaque les Vers à Soie. $\quad$ 8०. Paris, 1838.

AUDOUIN (J. V.). Histoire des Insectes nuisibles à la Vigne.

4. Paris, 1842.

AUdoUin (J.V.). See Periodicals : London. Willughby Society; and ParIs. Annales.

AUDUBON (J. J.). Ornithological Biography.

5 vols. $8^{\circ}$. Edinburgh, 1831-39.

AUDUBON (J. J.). A Synopsis of the Birds of North America. 8․ Edinburgh and London, 1839.

AUDUBON (J. J.). The Birds of America: from drawings made in the United States and their territories.

7 vols. roj. $8^{\circ}$. New York, 1840-44.

AUDUBON (J. J.) and BACHMAN (J.). The Viviparous Quadrupeds of North America.

Text, 3 vols. roy. $8^{\circ}$. 1846-54. Atlas, 3 vols. fol. 1845-48.

AUDUBON (J. J.) and BACHMAN (J.). The Quadrupeds of North America. $\quad 3$ vols. $8^{\circ}$. New York, 1854. 
AUTENRIETH (H. F.). Ueber das Gift der Fische.

8. Tübingen, 1833.

AZARA (F. d'). Histoire Naturelle des Quadrupedes de Paraguay. 2 vols. $8^{\circ}$. Paris, 1801.

AZARA (F. d'). Selections from the Natural History of Quadrupeds of Paraguay and the River la Plata. From the Spanish by W. P. Hunter.

8. London, 1837.

AZARA (F. d'). See Hartlaub (G.).

B.

B. (H.). Die Fischereiindustrie Norwegens von H. B. [BAARS]

8. Bergen, 1880.

BACCIO (A.). De Venenis, et antidotis prolegomena. De Canis rabiosi morsu.

$4^{\circ}$. Romce, 1586.

BACH (M.). Käferfauna für Nord- und Mitteldeutschland. 4 vols. $8^{\circ}$. Coblenz, 1851-67.

BACH (M.). Systematisches Verzeichniss der Käfer Deutschlands, gehörend zur Käferfauna.

[From vol. iv., last title.] $\quad 8^{\circ}$. Coblenz, 1866.

BACHMAN (J.). See Audubon (J. J.).

BACHMANN (F.). Nicolaus Georg Gerens ConchylienCabinet. $4^{\circ}$. Lïneburg, 1830.

BÄCHTOLD (J. J.). Untersuchungen über die Giftwerkzeuge der Schlangen.

$4^{\mathrm{o}}$. Tübingen, 1843.

BADHAM (D.). Insect Life.

12․ Edinburgh \& London, 1845.

BAEDEKER (F. W. J.). Die Eier der Europäischen Vögel. $\quad 4$ vols. in 2. fol. Leipzig \& Iserlohn, 1855.

BAER (A. von). Kaspische Studien.

8. St. Petershurg, 1859. 
BAER (K. E. v.). Berichte über die Zoographia RossoAsiatica von Pallas.

$4^{\circ}$. Königsberg, 1832.

BAER (K. E. v.). Anatomische und zoologische Untersuchungen über das Wallross (Trichechus rosmarus).

$4^{\circ}$. St. Petersburg, 1836.

BAER (K. E. v.). Ueber doppelleibige Missgeburten oder organische Verdoppelungen in Wirbelthieren.

$4^{\circ}$. St. Petersburg, 1845.

BAER (K. E. v.). Materialien zu einer Geschichte des Fischfanges in Russland und den angränzenden Meeren.

8.. St. Petersburg, 1853.

BAER (K. E. v.). Ueber Papuas und Alfuren.

$4^{\circ}$. St. Petersburg, 1859.

BAER (K. E. v.). Crania selecta ex Thesauris Anthropologicis. 4\%. Petropoli, 1859.

BAER (K. E. v.). Die Makrokephalen im Boden der Krym und Österreichs.

$4^{\circ}$. St. Petersburg, 1860.

BAER (K. E. v.). Das Fünfzigjährige Doctor-Jubiläum des Geheimraths K. E. v. BAER. $4^{\circ}$. St. Petersburg, 1865.

BAER (K. E. v.). Nachrichten über Leben und Schriften des K. E. v. BaEr. $\quad 8^{\circ}$. St. Petersburg, 1866.

BAER (K. E. v.). Reden gehalten in wissenschaftlichen Versammlungen. Theil III. Historische Fragen.

8. St. Petersburg, 1873.

BAER (K. E. v.). Entwickelt sich die Larve der einfachen Ascidien nach dem Typus der Wirbelthiere?

$4^{\circ}$. St. Petersburg, 1873.

BAER (K. E. v.). See Stieda (L.).

BAIKIE (W. B.) and HEDDLE (R.). Historia Naturalis Orcadensis : A Catalogue of the Mammalia and Birds observed in the Orkney Islands. $\quad 8^{\circ}$. Edinburgh, 1848. 
BAILEY (J.S.). The Natural History of AEgeria pictipes. 8. Albany, n. d.

BAILLY (J. B.). Observations sur les mœurs et les habitudes des Oiseaux de la Savoie.

8. Chambéry, 1847.

BAILLY (J. B.). Ornithologie de la Savoie.

4 vols. in 2. $8^{\circ}$. Paris, 1853-54.

BAIRD (S. F.). On the Serpents of New York.

8. Albany, 1854.

BAIRD (S. F.). Catalogue of North-American Mammals, chiefly in the Museum of the Smithsonian Institution.

$4^{\text {o. }}$ Washington, 1857.

See Musedm : Suithsonian Institution.

BAIRD (S. F.). Catalogue of North-American Birds, chiefly in the Museum of the Smithsonian Institution.

See Museum : Suithsonian Institution.

$4^{\circ}$. Washington, 1858-59.

BAIRD (S. F.). Mammals of North America.

4. Philadelphia, 1859.

BAIRD (S. F.). Reptiles from the Exploring Expedition for a Railroad-route from the Mississippi River to the Pacific Ocean.

See Survers and Explorations: Washington.

BAIRD (S. F.). Review of American Birds.

8. Washington, 1864.

BAIRD (S. F.). Report on the Condition of the Sea Fisheries of the South Coast of New England in 1871 and 1872.

See Fisheries : Washington.

8. Washington, 1873.

BAIRD (S. F.), CASSIN (J.), and LAWRENCE (G. N.). The Birds of North America.

2 vols. $4^{\circ}$. Philadelphia, 1860. 
BAIRD (S. F.), BREWER (T. M.), and RIDGWAY (R.). A History of North-American Birds.

3 vols. $8^{\circ}$. Boston, 1874 .

BAIRD (S. F.) and GIRARD (C.). Catalogue of North American Reptiles.

See Museum : Sumthomiay Irstitutiox.

BAIRD (W.). The Natural History of the British Entomostraca, $8^{\circ}$. London, 1850.

See Ray Society.

BAIRD (W.). Catalogue of the Species of Entozoa in the Collection of the British Museum. 12 . London, 1853. See Museum (British).

BAKER (S. W.). The Rifle and the Hound in Ceylon. $8^{\circ}$. London, 1854.

BAKER (S. W.). Eight Years' Wanderings in Ceylon.

8. London, 1855.

BAKKER (G.). Osteographia Piscium.

Text, $8^{\circ}$. Icones, $4^{\circ}$. Groninga, 1822.

BALBIANI (G.). Leçons sur la Génération des Tertébrés. Ed. HeNTegur.

8. Paris, 1879.

BALBIAXI (G.). Leçons sur les Sporozoaires.

8. Paris, 1881.

BALDWIN (J. H.). The Large and Small Game of Bengal, and the North-western Provinces of India.

8. London, 1877.

BALFOUR ( ? ). Birds of Eastern and Southern Asia. Privately printed. $8^{\circ}$. Calcutta, n. d.

BALFOUR ( ? ). Fisheries and Eishes of Eastern and Southern Asia. $\quad$ Prirately printed. $8^{\circ}$. Calcutta, n. d.

BALFOUR (F. M.). Studies from the Morphological Laboratory in the University of Cambridge.

8. London, 1880. 
BALFOUR (F. M.). A Treatise on Comparative Embryo$\log \mathrm{y}$. 2 vols. $8^{\circ}$. London, 1880-81.

BALL (R.). Natural History of the Seals (Phocidce) of the British Islands. 4\%. London, 1829.

BALL (R.). On the Phocida inhabiting the Irish Seas. $4^{\circ}$. Dublin, 1838.

BALL (R.). On Phoca vitulina. $4^{\text {o. }}$ Ipswich, 1847.

BALY (J. S.). Catalogue of Hispidce in the Collection of the British Museum.

8. London, 1858.

See Museum (British).

BAMBEKE (C. van). See Periodicals : Ghent.

BARBUT (J.). The Genera Insectorum of Linnœus exemplified....

(Additional title in French.) $\quad 4^{\circ}$. London, 1781.

BARBUT (J.). The Genera Vermium of Linnæus exemplified.... $4^{\circ}$. London, 1788.

BargEO (P. A.). See Tuano (J. A.).

BARKOW (H.). Syndesmologie der Vögel.

fol. Breslau, 1856.

BARKOW (H.). Anatomisch-physiologische Untersuchungen vorzüglich über das Schlagadersystem der Vögel.

8. Leipzig, 1830.

BARKOW (H. C. L.). Das Leben der Walle in seiner Beziehung zum Athmen und zum Blutlauf.

$4^{\circ}$. Breslau, 1862.

BARKOW (H. C. L.). Comparative Morphologie des Menschen und der menschenähnlichen Thiere.

Vols. I.-VI. fol. Greifswald \& Brestcu, 1862-75.

BARKOW (J. C. L.). Disquisitiones circa originem et decursum arteriarum Mammalium.

$4^{\circ}$. Lipsice, 1829 .

ZOOL. CAT. 
BARLOW.(F.). Avium Tabulæ Celeberrimæ (13).

obl. $4^{\circ}$. London, n. d.

BARRINGTON (R. M.). See Brown (J. A. H.) and others.

BARROIS (J.). Recherches sur l'Embryologie des Bryozoaires. $4^{\circ}$. Lille, 1877.

BARROIS (J.). Mémoire sur l'Embryologie des Némertes. $4^{\circ}$. Paris, 1877.

BARROW (J. H.). See Landseer (T.).

BARTHELEMY-SAINT-HILAIRE(J.). See ARIstoteles.

BARTON (B. S.). Fragments of the Natural History of Pennsylvania.

Part I., all published. $\quad 4^{\circ}$. Philadelphia, 1799.

BArton (B. S.). See Periodicals: London. Willughby Society.

BASCHIERI (A.). See Gazzadi (D.).

BASTER (J.). Opuscula Subseciva, Observationes miscellaneas de Animalculis et Plantis....

2 vols. $4^{\circ}$. Haarlemi, 1759-65.

BASTER (J.). Natuurkundige Uitspanningen, ... . over sommige Zee-planten en Zee-insecten....

2 vols. in 1. $4^{\circ}$. Haarlem, 1762-65.

BASTIAN (A.). Das Beständige in den Menschenrassen und die Spielweite ihrer Veränderlichkeit.

8. Berlin, 1868.

BATE (C. S.). Catalogue of Amphipodous Crustacea in the Collection of the British Museum. 80. London, 1862.

See Musedm (British).

BATE (C. S.) and WESTWOOD (J. O.). A History of the British Sessile-eyed Crustacea. 2 vols. $8^{\circ}$. London, 1868. 
BATES (H. W.). The Naturalist on the River A mazons. 2 vols. $8^{\circ}$. London, 1863.

BATES (H. W.) and CLARK (H.). A Catalogue of Phytophaga, with an Appendix. 8. . London, 1866.

Bates (H. W.). See Biologia Centrali-Americana.

BATTARA (J. A.). See Bonanni (P. P.).

BAUDELOT (E.). Recherches sur le Système nerveux des Poissons. fol. Paris, 1883.

BaUdi (T.). See Periodicals: Turin.

BAUDRIMONT (A.) et MARTIN-SAINT-ANGE (G. J.). Du Déreloppement du Fœtus. $4^{\circ}$. Paris, 1850.

BAUME (R.). Odontologische Forschungen. Theil I. 8. Leipzig, 1882.

BAUMERT (F. M.). Chemische Untersuchungen über die Respiration des Schlammpeizgers (Cobitis fossilis).

$4^{\circ}$. Breslau, 1855.

BAUR (H.). De Mammalibus Mergentibus.

$4^{\circ}$. Tubingce, 1832 .

BAVAY (A.). Catalogue des Reptiles de la Nouvelle Caledonie. $4^{\circ}$. Caen, 1872.

'BEAGLE.' The Zoology of the Vojage of H.M.S. 'Beagle' under the command of Capt. Fitzror, 1832-36.

Fossil Mammalia. By R. Owen.

$4^{\circ}$. London, 1840-42.

Mammalia. By G. O. Waterhouse.

Birds. By J. Gould and C. Darwin.

Fishes. By L. Jenyns.

Reptiles. By T. BeLL.

BEAN (T. H.). See 'Corwin.'

BEAUMONT (E. H. de). Études sur La Pisciculture.

8.. Paris, 1868. 
BEALE (T.). Natural History of the Sperm Whale.

$8^{\circ}$. London, 1839.

BEAVAN (R.). Handbook of the Freshwater Fishes of India. $8^{\circ}$. London, 1877.

BECHE (H. de la). The Geological Observer.

8. London, 1851.

BECHSTEIN (J. M.). Gemeinniitzige Naturgeschichte Deutschlands. 4 vols. $8^{\circ}$. Leipzig, 1789-95.

BECHSTEIN (J. M.). Naturgeschichte der Stubenthiere. 12.. Gotha, 1797.

BECHSTEIN (J. M.). Naturgeschichte der Stubenvögel. $8^{\circ}$. Halle, 1840.

BECHSTEIN (J. M.) und SCHARFENBERG (G. L.). Vollständige Naturgeschichte der schädlichen Forstinsekten. $4^{\circ}$. Leipzig, 1804-05.

BECK (G.). Ueber die Haftscheibe der Echeneis remora. $8^{\circ}$. Schaffhausen, 1879 .

BECK (H.). Index Molluscorum Musei Prince Christiani Frederici.

$4^{\circ}$. Hafnice, 1837.

BEDRIAGA (J. ₹.). Ueber die Entstehung der Farben bei den Eidechsen.

$8^{\circ}$. Jena, 1874.

BEECHEY (F. W.). See ' Blossom.'

BEHN (W. F. G.). See Cuvier (G.).

BEKKER (C. W.). See Tedtsche Ornithologie.

BELANGER (C.). Voyage aux Indes Orientales par le nord de l'Europe (Zoologie).

Text, $8^{\circ}$. Atlas, $4^{\circ}$. Paris, 1834.

Mammifères. Par I. Geoffroy St. Hilaire.

Oiseaux, Reptiles et Zoophytes. Par R. P. Lesson.

Poissons. Par A. Valenciennes.

Mollusques. Par G. P. Deshayes.

Insectes. Par F. E. Guérin. 
BELANY (J. C.). A Treatise on Falconry.

8. Berwick-on-Tweed, 1841.

BELCHER (Sir E.). See 'SAMaRarg.'

BELL (F. J.) and LANKESTER (E. Ray).

See Gegenbadr (C.).

BELL (T.). A History of British Quadrupeds, including the Cetacea. $\quad 8^{\circ}$. London, 1837.

Second Edition by R. F. Tomes and E. R. Alston.

8. London, 1874.

BELL (T.). A History of British Reptiles.

8.. London, 1839.

BELL (T.). A History of the British Stalk-eyed Crustacea.

$8^{\circ}$. London, 1853.

BELL (T.). Catalogue of Crustacea in the Collection of the British Museum. Leucosiadce. 12 . London, 1855. See Museum (British).

BELL (T.). A MIonograph of the Testudinata, with text by Dr. J. E. GraT.

$4^{\circ}$. London, 1872.

BELL (T.). See 'Beagle.'

BELL (T.). See Sotwerby (G.) and Lear (E.).

BELLARDI (L.). Saggio di Ditterologia Messicana.

Parts I.-III. 4\%. Torino, 1859-62.

BELLARS (H. J.). Illustrated Catalogue of British Land and Freshwater Shells. $\quad 8^{\circ}$. Chester, 1858.

BELLERMANN (J. J.). Ceber das bisher bezweifelte Daseyn des Rattenköniges. $\quad 12^{\circ}$. Berlin, 1820.

BELLESME (J. de). Recherches expérimentales sur les fonctions du balancier chez les Insectes Diptères.

8.. Paris, 1878. 
BELLONIUS (P.). De Aquatilibus, Libri duo.

obl. $8^{\circ}$. Parisiis, 1553.

BENECKE (B.). Fische, Fischerei, und Fischzucht in Ost- und Westpreussen.

8․ Königsberg, 1881.

BENEDEN (Е. van). See Periodicals : Ghext.

BENEDEN (P. J. ran). Exercices Zootomiques.

$4^{\circ} . \quad$ Biruxelles, 1839.

BENEDEN (P. J. $\mathrm{van})$. Recherches des Bryozoaires qui habitent la côte d'Ostende. $\quad 4^{\circ}$. Bruxelles, 1845.

BENEDEN (P. J. van). Les Vers cestoïdes ou Acotyles. $4^{\circ}$. Bruxelles, 1850 .

BENEDEN (P. J. ran). Iconographie des Helminthes ou des Vers Parasites de l'Homme. Vers Cestoïdes.

$4^{\circ}$. Louvain et Paris, 1859.

BENEDEN (P. J. ran). Recherches sur les Squalodons. $4^{\circ}$. Bruxelles, 1865.

BENEDEN (P. J. van) and GERTAIS (P.). Ostéographio des Cétacés.

Parts I.-VIII. Texte, $4^{\circ}$. Planches, fol. Bruxelles, n.d. [In progress.]

BENGEL (E.). See RApp (T. L.).

BENNETT (E. T.). See 'Blossour.'

BENNETT (E. T.). The Tower Menagerie.

$8^{\circ}$. London, 1829.

BENNETT (E. T.). The Gardens and Menagerie of the Zoological Society delineated.

2 vols. $8^{\circ}$. London, 1830-31.

BENNeTT (G.). See Mrsedx (Adstraliax).

BENNETT (J. W.). Fishes of Ceylon. 4. London, 1830. 
BENOIT (L.). Ornitologia Siciliana. $\quad 8^{\circ}$. Messina, 1840.

BENOIT (L.). See Aradus (A.).

BENOIT (L.) \& TIBEKI (N.). Illustrazione de' Testacei Estramarini. Testacei marini del Iediterraneo di N. Tiberi. $4^{\circ}$. Napoli, 1857-62.

BERCE (E.). Faune Entomologique Française. Lépidoptères.

6 vols. $12^{\circ}$. Paris, 1867-78.

BERCHON (P. de F.). Les Fonds de la Mer.

$8^{\circ}$. Bordeaux, 1867.

BERENDT (G. C.). Die im Bernstein befindlichen Organischen Reste der Vorwelt.

fol. Berlin, 1845-56.

BERG (C.). Hemiptera Argentina.

8.. Bonarice \& Hamburgo, 1879.

BERGEN (C. A. de). Classes Conchyliorum.

$4^{\circ}$. Norimbergce, 1760.

BERGH(R.). Contributions to a Monograph of the genus Fiona. $8^{\circ}$. Copenhagen, 1859.

BERGH (R.). Phidiana lynceus og Ismaila monstrosa. 8․ Kjöbenthavn, 1867.

BERGH (R.). Anatomische Untersuchung der Pleurophyllidia formosa.

8०. Wien, 1869.

BERGH (R.). Svar og Gjensvar paa Etatsr. I. Steenstrups. $8^{\circ}$. Kjöbentavn, 1871.

BERGH (R.). Beiträge zur Kenntniss der Mollusken des Sargassomeeres.

$8^{\circ}$. Wien, 1871 .

BERGH (R.). Ueber eine grönländische Aplysie.

$8^{\circ}$. Wien, 1872.

BERGH (R.). Beiträge zur Kenntniss der Aeolidiaden.

80. Wien, 1874. 
BERGH (R.). Tilfælde af Skorpefnat.

8․ Kjöbenhavn, 1874.

BERGH (R.). See Surveys and Explorations : Germany.

BERGMANN (E.) \& LEUCKART (R.). Anatomisch-physiologische Uebersicht des Thierreichs. Vergleichende Anatomie und Physiologie. $\quad 8^{\circ}$. Stuttgart, 1852.

BERGMANN (J.), Inauguralfrage: Was die Thiere gewiss nicht, \&c. sm. $8^{\circ}$. Mainz, 1784.

BERGSOE (V.). Philichthys Xiphice.

$8^{\circ}$. Kjöbenhavn, 1864.

BERGSTRÄSSER (J. A. B.). Nomenclatur und Beschreibung der Insecten in der Grafschaft Hanau-Münzenberg.

$4^{\circ}$. Hanau, 1778-80.

BERGSTRAESSER (J. A. B.). Icones Papilionum Diurnorum.

$4^{\circ}$. Hanovice, 1781.

BERGSTRAESSER (H. W.). Sphingum Europaearum Larvae. 4․ Hanau, 1782.

BERKELEY (G. F.). The English Sportsman in the Western Prairies. 8. London, 1861.

BERKENHOUT (J.). Synopsis of the Natural History of Great Britain and Ireland. 2 vols. $8^{\circ}$. London, 1789.

BERLAK (S.). Symbola ad Anatomiam Vesicæ Natatoriæ Piscium. $8^{\circ}$. Regimontii, 1834.

BERLESE (A.). Acari, Miriapodi, et Scorpioni. Fasc. I.-IX. $8^{\circ}$. Padova, 1882-83. [In progress.]

BERNARDI (A.). Monographie du Genre Conus. 8. Paris et Leipzig. 
BERNARDI (A. C.). Monographie des genres Galatea et Fischeria. $4^{\circ}$. Puris, 1860.

BERNSTEIN (H. A.). De Anatomia Corvorum. Pars I. Osteologia.

$8^{\circ}$. Vratistavice, 1853.

BERTHELOT (S.). Do la Pêche sur la côte Occidentale d'Afrique.

$8^{\circ}$. Paris, 1840.

BERTHELOT (S.). Études sur les Pêches Maritimes dans la Méditerranée et l'Océan.

$8^{\circ}$. Paris, 1869.

BERTHELOT (S.). Titalité des Mers.

8. . Paris \& Marseille, 1878.

BERTHOLD (A. A.). Ueber verschiedene neue oder seltene Amphibienarten. $\quad 4^{\circ}$. Göttingen, 1842.

BERTHOLD (A. A.). Über verschiedene neue oder seltene Reptilien aus Neu Granada und Crustaceen aus China.

$4^{\circ}$. Gottingen, 1846.

BERTHOLD (G.). See Fadra dxd Flora des Golfes von Neapel.

BERTOLONI (G.). Historia Lepidopterorum Agri Bononiensis.

$4^{\circ}$. Bononice, 1844.

BERTRAM (J. C.). See Fisheries : Londox.

BESEKE (J. M. G.). Beytrag zur Naturgeschichte der Tögel Kurlands. sm. $8^{\circ}$. Berlin, 1792.

BETTA (E. de). Di una Nuova Conchiglia virente indigena della provincia Veronese (Helix Pollinii).

8. Verona, 1849.

BETTA (E. de). Erpetologia delle provincie Venete e del Tirolo meridionale.

8.. Verona, $185 \%$.

BETTA (E. de). Catalogus systematicus rerum Naturalium in Museo extantium. Sectio I. Reptilia Europæ.

See Mosedu (Verona).

8. Verona, 1857. 
BETTA (E. de). Sulla Piscicultura. 8º . Verona, 1861.

BETTA (E. de). Ittiologia Veronese. $\quad 8^{\circ}$. Verona, 1862.

BETTA (E. de). Monografia degli Amfibi urodeli Italiani. $4^{\circ}$. Venеzia, 1864.

BETTA (E. de). Rettili ed Anfibi del regno della Grecia. 8. Venezia, 1868.

BETTA (E. de). Materiali per una Fauna Veronese.

$8^{\circ}$. Verona, 1863-70.

BETTA (E. de). Nuova serie di Note Erpetologiche per servire allo studio dei Rettili ed Anfibi d' Italia.

$8^{\circ}$. Tenezia, 1879.

BETTA (E. de). See Fauna d' Italia.

BetTany (G. T.). See Parker (W. K.).

BETTONI (E.). Storia Naturale degli Uccelli che nidificano in Lombardia. 2 vols. fol. Milano, 1865-68.

BEVAN (E.). The Honey Bee.

8․ London, 1838.

BEVAN (G. P.). British Industries. See Holdsworth (E. W. H.) and Young (A.).

BEWICK (T.). A General History of Quadrupeds.

$8^{\circ}$. Newcastle, 1790.

3rd edition. $8^{\circ}$. Newcastle, 1792.

7 th edition. $8^{\circ}$. Newcastle, 1820.

BEWICK (T.). Figures from Bewick's Quadrupeds, \&c.

4 vols. $4^{\circ}$. Newcastle, 1824.

BEWICK (T.). A History of British Birds.

2 vols. $8^{\circ}$. Newcastle, 1805. Another edition. 2 vols. $8^{\circ}$. Newcastle, 1821.

BEYRICH (H. E.). De Goniatitis in montibus Rhenanis occurrentibus. $4^{\circ}$. Berolini, 1837. 
BIANCONI (J. J.). Specimina Zoologica Mosambicana.

4\%. Bononice, 1850.

BIANCONI (J. J.). La Théorie Darwinienne. Lettre à M. C. Darwin. $8^{\circ}$. Bologne, 1874.

BIBLIOGRAPHIES.

AgAssiz. Bibliographia Zoologica.

4 vols. $8^{\circ}$. London, 1848.

Brnner (W. G.). Bibliography of N. American Conchology.

8. Washington, 1863-64.

BosGued. Bibliotheca Ichthyologica et Piscatoria.

8. Haarlem, 1874.

Carus and Exgelmany. Bibliotheca Zoologica.

2 rols. $8^{\circ}$. Leipzig, 1861.

Cavanna. Bibliografia Italiana. 8º. Firenze, 1880.

Dryander. Catalogus Bibliothecæ Historico-Naturalis Josephi Banks.

5 vols. $8^{\circ}$. London, 1798-1800.

Ebert. Bibliographical Dictionary.

4 vols. $8^{\circ}$. Oxford, 1837.

Engelmann. Bibliotheca Historico-Naturalis.

8. Leipzig, 1846.

Geographical Societr, Catalogue of the Library with 2 Supplements.

$8^{\circ}$. London, 1865-80.

$\mathrm{H}_{A G E N}$. Bibliotheca Entomologica.

2 vols. in $1.8^{\circ}$. Leipzig, 1862-63.

Hoppe. Katalog der Deutscher Literatur, 1801-68.

8. St. Petersburg, 1871.

JANSE. Catalogus der Bibliotheek te Amsterdam.

roy. $8^{\circ}$. Amsterdam, 1881.

Linnean Societr, Catalogue of the Library. Parts I., II., III.

$8^{\circ}$. London, 1866-77.

Lowndes. Bibliographer's Manual.

4 vols. $8^{\circ}$. London, 1834.

Poole. Index to Periodical Literature.

$4^{\circ}$. Boston, 1882.

Quaritch. General Catalogue of Books.

Parts I.-III. $8^{\circ}$. Lonclon, 18ع0-82. 
BIBLIOGRAPHIES (continued).

Scudder. Catalogue of Scientific Serials.

roy. $8^{\circ}$. Cambrilge, U.S., 1879.

Westwood and Satchedr. Bibliotheca Piscatoria.

8. London, 1883.

Zoological Societr, Catalogue of the Library and Supplement.

$8^{\circ}$. Lonlon, 1880-83.

BLDDER (T. H.). Vergleichend-anatomische und histologische Untersuchungen über die männlichen Geschlechtsund Harnwerkzeuge der nackten Amphibien.

$4^{\circ}$. Dorpat, 1846.

BIELZ (E. A.). Fauna der Wirbelthiere Siebenbürgens.

8o. Hermannstadt, 1856.

BIELZ (E. A.). Verzeichniss der Mollusken- und Conchylien-Sammlung. $\quad 8^{\circ}$. Hermannstadt, 1865.

BIENERT (Th.). Lepidopterologische Ergebnisse einer Reise in Persien in 1858 und 1869. 8 $^{\circ}$ Leipzig, 1869.

BILHARZ (T.). Das electrische Organ des Zitterwelses. $4^{\circ}$. Leipzig, 1857.

BILLBERG (G. J.). Monographia Mylabridum.

8.. Holmice, 1813.

BILLbERG (G. J.). See Svensi Zoologi.

BINGLEY (W.). Animal Biography.

2nd edition. 3 vols. $8^{\circ}$. London, 180 t.

BINNEY (A. \& W. G.). The Terrestrial Air-breathing Mollusks of the United States.

Edited by A. A. Gould. Supplement by W. G. Binney. 4 vols. $8^{\circ}$. Boston, 1851-59.

BINNEY (W. G.). Bibliography of North-American Conchology, previous to the year 1860 .

$8^{\circ}$. Washington, 1863-64. 
BINNEY (W. G.). See SAY (T.).

BINNEY (W. G.), BLAND (T.), and TRYON (G. W.)

Land and Fresh-water Shells of North America.

$8^{\circ}$. Washington, 1865-73.

See Suithsonian Irstitution : Miscell. Coll.

BIOLOGIA CENTRALI-AIIERICANA. Edited by F. DoCane Godman and Osbert Salvin. [Zoology.j

[In progress.] $4^{\circ}$. London, 1879-83.

Mammalia. By Edward R. Alston.

Aves. By Osbert Salvin and F. DoCane Goduran.

Reptilia, Amphibia, Pisces. By A. Günther.

Mollusca (Land and Freshmater). By E. ron Martens.

Crustacea (Freshwater Malacostraca). By T. H. HUXLEY.

Arachnida. By O. Pickard Cambridge.

Coleoptera. By H. W. Bates, H. S. Gorhan, Martin JACOBX, E. W. JANSON, D. Sharp, and C. O. WATERHOUSE.

Lepidoptera. By F. DuCane Godman, Osbert Salvin, and Herbert Druce.

Neuroptera. By R. M['Lachlan.

Orthoptera. By J. Wood-Mason.

Rhynchota. By W. L. Distant.

BIRDS. Tabular view of the Orders and Families of Birds. [Printed for the Society for Promoting Christian Knowledge, n. d.]

BISCHOFF (T. L. W.). Lepidosiren paradoxa.

$4^{\circ}$. Leipzig, 1840.

BISCHOFF (T. L. W.). Entwicklungsgeschichte des Kaninchen-Eies. $\quad 4^{\circ}$. Braunschweig, 1842.

BISCHOFF ('T. L. W.). Beweis der von Begattung unabhängigen periodischen Reifung und Loslösung der Eier der Säugethiere und des Menschen.

$4^{\circ}$. Giessen, 1844.

BISCHOFF (T. L. W.). Entwicklungsgeschichte des Hunde-Eies. $4^{\circ}$. Braunschweig, 1845.

BISCHOFF (T. L. W.). Historisch-Kritische Bemerkungen zu den neuesten Mittheilungen über die erste Entwicklung der Säugethiereier.

8. München, 187\%. 
BLACKWALL (J.). Researches in Zoology.

2nd edition. $8^{\circ}$. London, 1873.

BLACKWALL (J.). Britain and Ireland.

See Ray Soctety.
A History of the Spiders of Great fol. London, 1860-64.

BLAINTILLE (H. M. D. de). De l'Organisation des Animaux, ou Principes d'Anatomie comparée. 8․ Paris, 1822.

BLAITVILLE (H. M. D. de), Manuel de Malacologie et de Conchyliologie.

Texte et Planches. $8^{\circ}$. Paris, 1825-27.

BLAINVILLE (H. M. D. de). Manuel d'Actinologie ou de Zoophytologie. Texte et Atlas. 80. Paris, 1834.

BLAINVILLE (H. M. D. de). Ostéographie, ou description iconographique comparée du squelette et du système dentaire des Mammifères.

Text, 4 vols. $4^{\circ}$. Atlas, 4 vols. fol. Paris, 1839-64.

BLainville (H. M. D. de). See Faune Française.

BLANCHARD (E.). Histoire des Insectes, métamorphoses en général.

2 vols. $8^{\circ}$. Paris, 1845.

BLANCHARD (E.). Catalogue des Coléoptères du Muséum d'Histoire naturelle de Paris. See Museum (Paris).

BLANCHARD (E.). La Zoologie Agricole. 4º Paris, 1854.

BLATCHARD (E.). Les Poissons des Eaux douces de la France. 8. Paris, 1880.

BLANCHARD (E.). See Sodance (C, de).

BLANCK (A.). Die Fische der Seen und Flüsse Mecklenburgs.

80. Schwerin, 1881.

BLAND (T.). See Birvex (W. G.). 
BLANFORD (W. T.). See Surveys and Explorations : YARKAND.

BLASIUS (G.). Zootomiæ seu Anatomes variorum animalium. 12. Amstelodami, 1676-77.

BLASIUS (G.). Anatome Animalium.

4․ Amstelodami, 1681.

BLASIUS (J. H.). Naturgeschichte der Säugethiere Deutschlands und der angrenzenden Länder von Mitteleuropa.

$8^{\circ}$. Braunschweig, 1857.

BLASIUS (J. H.). A List of the Birds of Europe. Reprinted from the German, with the author's corrections.

8. London, 1862.

BLEEKER (P.). Atlas Ichthyologique.

7 vols. fol. Amsterdam, 1862-77.

BLEEKER (P.), SCHLEGEL (H.), \& WESTERMAN (G. F.). Nederlandsch Tijdschrift voor de Dierkunde.

4 vols. roy. $8^{\circ}$. Amsterdam, 1863-73.

BLEekER (P.). See Periodicals : Amsterday.

BLEEKER (P.). See Pollen et Van Dam.

BLOCH (M. E.). Abhandlung von der Erzeugung der Eingeweidew ïrmer. $4^{\circ}$. Berlin, 1782.

BLOCH (M. E.). Oeconomische Naturgeschichte der Fische Deutschlands.

Text, $4^{\circ}$. Atlas, fol. Berlin, 1782-85.

BLOCH (M. E.). Naturgeschichte der Ausländischen Fische.

Text, 3 vols. $4^{\circ}$. Atlas, 3 vols. fol. Berlin, 1785-95.

BLOCH (M. E.). Systema Ichthyologiæ iconibus CX. illustratum. Ed. J. G. Schneider.

2 vols. $8^{\circ}$. Berolini, 1801. 
BLOCH (M. E.). Von den rermeinten doppelten Zeugungsgliedern der Rochen und Haye.

sm. $8^{\circ}$. [Berlin, 1785.]

BLOOMFIELD (J. C.). See Fisheries : London.

'BLOSSOM.' The Zoology of Captain Beechey's Voyage in H.M.S. 'Blossom' in the years 1825-28.

Mammalia. By J. Richardson.

Ornithology. By N. A. Vigors.

Fishes. By G. T. Lay and E. T. Bennetr.

Crustacea. By R. Owen.

Reptiles. By J. E. Gray.

Batrachians. By E. E. GraY.

Mollusca. By J. E. Grar.

Geology. By A. Coldie.

BLUMBERG (C.). Ueber den Bau des Amphistoma conicum.

$4^{\circ}$. Dorpat, 1871.

BLUMENBACH (J. F.). Specimen physiologiæ comparatæ inter animantia calidi sanguinis vivipara et oripara. $4^{\circ}$. Gottingce, 1789 .

BLUMENBACH (J. F.). Abbildungen naturhistorischer Gegenstände. 8. Göttingen, 1810.

BLUMENBACH (J. F.). De Generis humani varietate nativa liber.

8. Göettingae, 1776.

BLUMENBACH (J. F.). Decas $\left(1^{\mathrm{a}}, 2^{\mathrm{da}}, 3^{\mathrm{a}}, 4^{\mathrm{ta}}, 5^{\text {ta }}, 6^{\text {ta }}\right)$ Collectionis suæ Craniorum. $\quad 4^{\circ}$. Gottinge, 1790-1820.

BLUMENBACH (J. F.). Manuel d'Histoire Naturelle, traduit de l'Allemand par S. ARTACD.

2 vols. $8^{\circ}$. Metz, 1803.

BLUMENBACH (J. F.). Beyträge zur Naturgeschichte. 12․ Göttingen, 1806.

BLUMENACH (J. F.). Nora Pentas Collectionis sur Craniorum.

See Blumenbach (J. F.). Decas, \&c.

BLUMBENACH (J. F.). See Neergaard (J. W.). 
BLYTH (E.). Zoology of the Tenasserim Provinces.

8. Calcutta, 1843.

BLYTH (E.). Catalogue of the Birds in the Museum of the Asiatic Society. $\quad 2$ vols. $\quad 8^{\circ}$. Calcutta, 1849.

BLYTH (E.). Catalogue of the Mammalia in the Museum of the Asiatic Society.

8․ Calcutta, 1863.

BLYTH (E.). Catalogue of the Mammals and Birds of Burma.

80. Hertford, 1875.

BLYTH (E.). The Natural History of the Cranes. Reprinted by W. B. Tegetmeier. $\quad 8^{\circ}$. London, 1881.

BÖBER (J. de). Description de quelques nouvelles espèces de Papillons découverts en Sibérie. $\quad 4^{\circ}$. Moscou, 1809.

BOCAGE (J. V. B. du). Noticia de um novo genero de Mammiferos Insectivoros da Africa occidental.

$4^{\circ}$. Lisboa, 1865.

BOCAGE (J. V. B. du). Ornithologie d'Angola.

$8^{\circ}$. Lisbonne, 1881.

BOCAGE (J. V. B. du) et CAPELLO (F. de B.). Notes pour servir à l'Ichthyologie du Portugal. Poissons Plagiostomes. (Second title in Portuguese.) $4^{\circ}$. Lisbonne, 1866.

BOCCIUS (G.). A treatise on the managoment of Freshwater Fish.

8. London, 1841.

BOCCIUS (G.). Fish in Rivers and Streams.

8. London, 1848.

BOCHART (S.). Hierozoicon, sive bipartitum opus de Animalibus S. Scripturæ.

fol. Francofurti, 1675.

Another edition. fol. Lugduni Batavorum, 1712.

BOCKING (A.). De Rhea americana. $8^{\circ}$. Bonnce, 1863.

BOCOURT. See Surveys and Explorations : France.

BODDAERT (P.). De Testudine cartilaginea. Over de Kraakbeenige Schildpad. $\quad 4^{\circ}$. Amstelodami, 1770. 
BODDAERT (P.). De Rana bicolore. Over den Tweekoleurigen Kikvorsch.

$4^{\circ}$. Amstelodami, 1772.

BODDAERT (P.). Natuurkundige Beschouwing der Dieren. $8^{\circ}$. Utrecht, 1778.

BODDAERT (P.). Tables des Planches enluminéez d'histoire naturelle. (Reprinted by E. B. Tegetmeier.) roy. $8^{\circ}$. London, 1874.

BOECK (A.). De Skandinaviske og arktiske Amphipoder. 4\%. Christiania, 1872-76.

BOETTCHER (A.). Neue Untersuchungen iiber die Rothen Blutkörperchen. $4^{\circ}$. St. Petersburg, 1876.

BOETTGER (O.). Beitrag zur Kenntniss der Reptilien Spaniens und Portugals. $\quad 8^{\circ}$. Offenbach am M., 1869.

BOETTGER (0.). Calamaria iris, n. sp.

8․ Offenbach am M., 1873.

BOETTGER (0.). Die Reptilien und Amphibien von Madagascar. $\quad 4^{\circ}$. Frankfurt am Main, 1877.

BOETTGER (0.). Clausilienstudien. $\quad 4^{\circ}$. Cassel, 1877.

BOETTGER(0.). Mittheilungen. Studien über neue oder wenig bekannte Eidechsen I. $\quad 8^{\circ}$. Frankfurt, 1879.

BOETTGER (0.). See Novitates Conchologicz.

BOHADSCH (J. B.). De quibusdam Animalibus Marinis eorumque proprietatibus.

40. Dresdce, 1761.

BOHADSCH (J. B.). Beschreibung einiger minderbekannten Seethiere und ihrer Eigenschaften.

4. Dresden, 1776.

BOHEMAN (C. H.). Monographia Cassididarum.

4 vols. $8^{\circ}$. Holmice, 1850-62.

BOHEMAN (C. H.). Insecta Caffrariæ.

2 vols. $8^{\circ}$. Holmice, 1848-5\%. Supplement by F̊̊nR巴Us, $1870-72$. 
BOHEMAN (C. H.). Catalogue of Coleopterous Insects in the Collection of the British Museum. Cassididae.

See Musedm (British).

$12^{\circ}$. London, 1856.

BOHEMAN (C. H.). See ' Eugenie.'

BOISDUVAL (J. A.). Monographie des Zygénides.

8. . Paris, 1829.

BOISDUVAL (J. A.). Faune Entomologique de Madagascar, Bourbon et Maurice. Lépidoptères.

8. Paris, 1834.

BOISDUVAL (J. A.). Genera et Index Methodicus Europæorum Lepidopterorum.

8. Paris, 1840.

BOISDUVAL (J. A.). Lépidoptères de la Californie.

$8^{\circ}$. Paris, 1852.

BOISDUVAL (J. A.). Essai sur l'Entomologie Horticole. 8. Paris, 1867.

BOISDUVAL (J. A.). Species général des Lépidoptères.

Vol. I. $8^{\circ}$. Paris, 1836.

BOISDUVAL (J. A.) \& GUENÉE (A.). Species général des Lépidoptères (Noctuelites). 3 vols. $\quad 8^{\circ} . \quad$ Paris, 1852.

Deltoüdes et Pyralites.

Uranides et Phalenites. 2 vols.

8.. Paris, 1854.

Hétérocères.

8. Paris, 1857.

8.. Paris, 1874.

BOISDUVAL (J. A.) \& LACORDAIRE (T.). Fauno Entomologique des environs de Paris. $12^{\circ}$. Paris, 1835.

BOISDUVAL (J. A.) \& LECONTE (J.). Histoire générale et Iconographie des Lépidoptères et des Chenilles de l'Amérique septentrionale.

$8^{\circ}$. Paris, 1833.

BOISDUVAL (J. A.), RAMBUR (P.), \& GRASLIN (A.). Collection Iconographique et Historique des Chenilles.

$8^{\circ}$. Paris, 1832 .

BOIS-REYMOND (E. du). See Sachs (C.). 
BOITARD (M. P.). Lө Jardin des Plantes. Introduction par J. JANIN.

8. Paris, 1842.

BOITARD et CORBIE. Les Pigeons.

8.. Puris, 1824.

BOJANUS (L. H.). Anatome Testudinis Europææ.

fol. Vilnce, 1819.

BOJANUS (L. H.). De Uro nostrate ejusque sceleto Commentatio. $4^{\circ}$. Bonnce, 1827.

BOLIVAR ('I.). Sinópsis de los Ortopteros de España y Portugal.

$8^{\circ}$. Madrid. 1878.

BOLL (F.). Beiträge zur vergleichenden Histiologie des Molluskentypus.

$8^{\circ}$. Bonn, 1869.

BOLTEN (J. F.). Nachricht von einer neuen Thierpflanze. 4. Hamburg, 1770.

BOLTON (J.). Harmonia Ruralis ; or, an Essay towards a natural history of British Song Birds.

(A new edition.) 2 vols. $4^{\circ}$. London, 1845.

BONAFOUS (M.). Traité de l'Éducation des Vers à Soie. 4th edition. $8^{\circ}$. Paris, 1840.

BONANNI (P. P.). Rerum Naturalium Historia..... exsistentium in Museo Kircheriano. Ed. J. A. Batrakra.

2 vols. fol. Romce, 1773 .

BONAPARTE (C. L.). American Ornithology.

4 vols. $4^{\circ}$. Philadelphia, 1825-33.

BONAPARTE (C. L.). Specchio Comparativo delle Ornithologie di Roma e di Filadelfia. $\quad 8^{\circ}$. Pisa, 1827.

BONAPARTE (C. L.). Osserrazioni sulla seconda edizione del "Regno Animale." $\quad 8^{\circ} . \quad B o l o g n a, 1830$.

BONAPARTE (C. L.). Saggio d'una distribuzione metodica degli Animali Vertebrati a sangue Freddo.

8․ Roma, 1832. 
BONAPARTE (C. L.). Iconografia della Fauna Italica. fol. Roma, 1832-41.

BONAPARTE (C. L.). Cheloniarum tabula analytica.

8. Roma, 1836.

BONAPARTE (C. L.). Synopsis Vertebratorum Systematis.

8. Romo, 1837.

BONAPARTE (C. L.). A Geographical and Comparative List of the Birds of Europe and North America.

8.. London, 1838.

BONAPARTE (C. L.). Amphibia Europaea ad systema nostrum vertebratorum ordinata.

$4^{\circ}$. Turin, 1839.

BONAPARTE (C. L.). Osservazioni sullo stato della Zoologia nel anno 1840-1841.

8. Firenze, 1842.

BONAPARTE (C. L.). Reports on the progress of Zoology and Botany, 1841-42.

$8^{\circ}$. Edinburgh, 1845.

Seo Ray Society.

BONAPARTE (C. L.). Catologo Metodico dei Mammiferi Europei. $4^{\circ}$. Milano, 1845.

BONAPARTE (C. L.). Spechio generale dei sistemi Erpetologico, Anfibiologico ed Ittiologico.

$4^{\circ}$. Milano, 1845.

BONAPARTE (C. L.). Catalogo Metodico dei Ciprinidi d'Europa. $4^{\circ}$. Milano, 1845.

BONAPARTE (C. L.). Catalogo Metodico dei Pesci Europei. $4^{\circ}$. Napoli, 1846.

BONAPARTE (C. L.). Revue Critique de l'Ornithologie Européenne. $12^{\circ}$. Bruxelles, 1850.

BONAPARTE (C. L.). Conspectus generum Avium. 8․ Lugduni Batavorum, 1850-57.

BONAPARTE (C. L.). Iconographie des Pigeons non figurés par Mde. KNIP dans l'ouvrage de Temminck.

fol. Paris, 1857. 
BONAPARTE (C. L.). See Sodance (C. de).

BONAPARTE (C. L.) \& SCHLEGEL (H.). Monographie des Loxiens.

4\%. Leiden, 1850.

BONAPARTE (C. L.). See Wilson (A.).

BONAVERI (G. F.). Della citta Comacchio delle sue lagune e pesche descrizione.

$4^{\circ}$. Incessena, 1761 .

- BONITE,' La. Voyage autour du Monde 1836-37. Commandée par Capt. L. VAILLANT.

Zoologie par Erdoux et SodLeyet.

Text, 2 vols. $8^{\circ}$. Atlas, fol. Paris, 1841-52.

BONIZZI (P.). I Colombi di Modena. 8. Modena, 1876.

BONNATERRE (l'Abbé). Encyclopédie Méthodique.

Poissons.

$4^{\circ}$. Paris, 1787.

BONOLA (G. B.). Della Bibliografia Malacologica Italiana. $8^{\circ}$. Milano, 1839.

BONSDORFF (E. J.). Finlands Tvåvingade Insekter (Diptera). 2 vols. $8^{\circ}$. Helsingfors, 1861-66.

BONVOULOIR (H. de). Essai Monographique des Throscides.

8.. Paris, 1859.

BOOTH (E. T.). Catalogue of the cases of Birds in the Dyke Road Museum, Brighton. $\quad 8^{\circ}$. Brighton, 1876.

BOOTH (E. T.). Rough Notes on the Birds obserred during twenty years in the British Islands.

Parts I.-IV. imp. 4\%. London, 1881. [In progress.]

BORELLI (J. A.). De Motu Animalium.

$4^{\circ}$. Lugduni Batavorum, 1685.

BORGGREVE (B.). Die Vogel-Fauna von Nord Deutschland.

$8^{\circ}$. Berlin, 1869 .

BORKHAUSEN (M. B.). Naturgeschichte der Europäischen Schmetterlinge. 5 vols. $8^{\circ}$. Frankfurt, 1788-94. 
BORKHAUSEN (M. B.). Deutsche Ornithologie.

Text and Atlas. fol. Darmstadt, 1837-41. [See Tedtsche Ornithologie.]

BORN (I. A.). Index Rerum Naturalium Musei Cæsarei Vindobonensis. Part I. Testacea. (Second title in German.)

$8^{\circ}$. Vindobonce, 1778.

BORN (I. A.). Testacea Musei Cæsarei Vindobonensis. fol. Vindobonce, 1780.

BORNE (M. von dem). See Frsheries : BerLin.

BOS (J. R.). Bijdrage tot de Kennis van de Crustacea hedriophthalmata van Nederland en zijne Kusten.

8. Groningen, 1874.

BOSC (L. A. G.). Histoire Naturelle des Vers.

2nd edition. 3 vols. $16^{\circ}$. Paris, 1830.

BOSC (L. A. G.). Manuel de l'Histoire Naturelle des Crustacés. Edit. par A. G. Deswarest.

2 vols. $16^{\circ}$. Paris, 1830 .

BOSCA (E.). Catalogo de los Reptiles y Anfibios observados en España, Portugal, é Islas Baleares.

8.. Madrid, 1877.

BOSE (F. C.). See Gutfleisch (V.).

BOSGOED (D. MI.). Bibliotheca Ichthyologica et Piscatoria.

$8^{\circ}$. Haarlem, 1874.

BOSMIAN (W.). Reise nach Guinea.

12. Hamburg, 1708.

BOUCHÉ (P. F.). Naturgeschichte der Insekten.

8. Berlin, 1834.

BOUCHER (C. F.). Recherches sur la Structure des Organes de l'Homme et des Animaux les plus connus. 8. Paris, 1848.

Boulart (R.). See Gervats (H.) et Boulart (R.). 
BOULENGER (G. A.). Catalogue of the Batrachia Salientia s. Ecaudata in the Collection of the British MIuseum. 2nd edition. $8^{\circ}$. London, 1882. See Musetm (British).

BOULENGER (G. A.). Catalogue of the Batrachia Gradientia s. Caudata and Batrachia Apoda.

See Moseum (British).

2nd edition. $8^{\circ}$. London, 1882.

BOULLENOIS (F. de). Conseils aux noureaux éducateurs de Vers à Soie. $8^{\circ}$. Paris, 1875.

BOURGUIGNAT (J. R.). Testacea novissima quæ F. de Saulcy in Itinere per Orientem annis 1850 et 1851 collegit.

$8^{\circ}$. Lutetice, 1852.

BOURGUIGNAT (J. R.). Catalogue raisonné des Mollusques terrestres et fluviatiles, recueillis par F. de Saulcy pendant son Voyage en Orient.

$4^{\circ}$. Paris, 1853.

BOCRGUIGNAT (J. R.). Aménités Malacologiques. 2 vols. $8^{\circ}$. Paris, $1856-60$.

BOURGUIGNAT (J. R.). Malacologie terrestre et fluviatile de la Bretagne. $8^{\circ}$. Paris, 1860.

BOURGUIGNAT (J. R.). Malacologie terrestre de l'ile du Chateau d'If, près de Marseille. $\quad 8^{\circ}$. Paris, 1860.

BOCRGUIGNAT (J. R.). Filum Ariadneum: Methodus Conchyliologicus Denominationis sine qua Chaos.

$8^{\circ}$. Paris, 1860.

BOLRGUIGNAT (J. R.). Etude synonymique sur les Mollusques des Alpes Maritimes publiés par A. Risso en 1826.

$8^{\circ}$. Paris, 1861.

BOURGUIGNAT (J. R.). Malacologie du Lac des QuatreCantons et de ses environs. $8^{\circ}$. Paris, 1862.

BOURGUIGNAT (J. R.). Les Spicilèges Malacologiques. 8. Paris, 1862.

BOURGUIGNAT (J. R.). Monographie du noureau genre Français Moitessieria. 8. Paris, 1863. 
BoURguignat (J. R.). Mollusques de San-Julia de Loria. 8. Paris, 1863

BoURGUignat (J. R.). Malacologie de la GrandeChartreuse. 8. . I'aris, 1864.

BOURGUIGNAT (J. R.). Malacologie d'Aix-les-Bains.

8. Paris, 1864.

BOURGUIGNAT (J. R.). Malacologie de l’Algérie.

2 vols. $4^{\circ}$. Paris, 1864.

BOURGUIGNAT (J. R.). Mollusques noureaux, litigieux ou peu connus.

8. Paris, 1863-68-70.

BOURGUIGNAT (J. R.). Mollusques terrestres et fluviatiles recueillis en Afrique dans le pays des Comalis Medjourtin.

8. Saint-Germain, 1881.

BOURGUIGNAT (J. R.). Miscellanées Italo-malacologiques.

8. Palermo, 1882.

BOURGUIGNAT (J. R.). Lettres malacologiques.

$8^{\circ}$. Paris, 1882.

BOURJOT (A.). Liste des Poissons que l'on rencontre le plus souvent au marché d'Alger.

8. Alger, 1870.

BOUSSUET (F.). De natura aquatilium carmen, in alteram partem universæ GULIELMI RoNDELeTII de aquatilibus historiæ.

$4^{\circ}$. Lugduni, 1558.

BOVI (R.). Dissertazione Italiana e Francese sopra la produzione de' Coralli. $8^{\circ}$. Firenze, 1769.

BOWDICH ('I. E.). An Analysis of the Natural Classification of Mammalia, for the use of Students and Travellers. Text and Atlas. $8^{\circ}$. Paris, 1821.

BOWDICH (T. E.). Elements of Conchology.

$8^{\circ}$. Paris and London, 1822.

BOWERBANK (J. S.). Observations on the Structure of Shell.

8. London, 1844. 
BOWERBANK (J. S.). A Monograph of the British Spongida. 4 rols. $8^{\circ}$. $1864-82$.

See $R_{A y}$ Societr.

BRABAZON (W.). The Deep-Sea and Coast Fisheries of Ireland.

BRACKEL (G. a). De cutis organo.

8. Dorpati Livonorum, 1858.

BRADY (G. S.). A Monograph of the British Copepoda.

See Ray Societr.

3 vols. $8^{\circ} .1878$.

BRADY (G. S.). See 'Challexger.'

BRADY (H. B.). Ueber einige arktische Tiefsee-Foraminiferen ..... $\quad 4^{\circ}$. Wien, 1881.

BRADY (T. F.). See Fisheries: Irelaxd.

BRAHM (N. J.). Insektenkalender für Sammler und Oekonomen. 3 vols. 12\%. Mainz, 1790-91.

BRANCSIK (C.). Die Käfer der Steiermark.

$8^{\circ} . \quad$ Graz, 1871.

BRaNdeN (C. van den). See Pertodicals: Brossels.

BRANDT (A.). Beiträge zur Entwicklungsgeschichte der Libelluliden und Hemipteren. $4^{\circ}$. St. Petersburg, 1869.

BRANDT (A.). Anatomisch-histologische Untersuchungen über den Sipunculus nudus L. $4^{\circ}$. St. Petersburg, 1870.

BRANDT (A.). Ceber Rhizostoma Cuvieri, Lamarck. 4\%. St. Petersburg, 1870.

BRANDT (A.). Ueber fossile Medusen.

40. St. Petersburg, 1871.

BRANDT (A.). Ueber die Haut der nordischen Seekuh (Rhytina borealis, Illig.). $\quad 4^{\circ}$. St. Petersburg, 1871. 
BRANDT (A.). Ueber die Eiröhren der Blatta (Periplaneta) orientalis. $4^{\circ}$. St. Petersburg, 1874.

BRANDT (A.). Ueber das Ei und seine Bildungsstätte.

8. Leipzig, 1878.

BRANDT (F.). See Surveys and Explorations: Russia.

BRANDT (J. F.). Ueber den Zahnbau der Steller'schen Seekuh (Rytina Stelleri). $\quad 4^{\circ}$. St. Petersburg, 1832.

BRANDT (J. F.). Naturhistorische Bemerkungen über Wurzelcochenille. $\quad 4^{\circ}$. St. Petersburg, 1834.

BRANDT (J. F.). Mammalium Rodentium exoticorum novorum descriptiones. $\quad 4^{\circ}$. St. Petersburg, 1835.

BRANDT (J. F.). Descriptiones et Icones Animalium Rossicorum. $\quad 4^{\circ}$. Petropoli, 1836.

BRANDT (J. F.). Ausführliche Beschreibung der von C. H. Mertens beobachteten Schirmquallen.

$4^{\circ}$. St. Petersburg, 1838.

BRANDT (J. F.). Spicilegia Ornithologica Exotica.

$4^{\circ}$. Petropoli, 1839.

BRANDT (J. F.). Beiträge zur Kenntniss der Naturgeschichte der Vögel. $\quad 4^{\circ}$. St. Petersburg, 1839.

BRANDT (J. F.). Symbolæ Sirenologicæ.

3 parts. $4^{\circ}$. Petropoli, 1846-68.

BRANDT (J. F.). Beiträge zur Kenntniss des Baues der innern Weichtheile des Lama (Auchenia lama).

4\%. St. Petersburg, 1847.

BRANDT (J. F.). Fuligulam (Lampronettam) fischeri descripsit. $4^{\circ}$. Petropoli, 1847.

BRANDT (J. F.). Collectanea Palæontologica Rossiæ Fasciculus I. Observationes ad Rhinocerotis tichorhini historiam spectantes. $4^{\circ}$. Petropoli, 1847. 
BRANDT (J. F.), Monograph of Acipenser ruthenus. $4^{\circ}$. St. Petersburg, 1852.

BRANDT (J.F.). Ueber die Wirbelthiere des nördlichen europäischen Russlands.

$4^{\circ}$. St. Petersburg, 1853.

BRANDT (J. F.). Untersuchungen über die Verbreitung des Tigers (Fetis tigris). $\quad 4^{\circ}$. St. Petersburg̈, 1856.

BRANDT (J. F.). Symbolæ ad Polypos Hyalochætides spectantes. fol. Petropoli, 1859.

BRANDT (J. F.). Observationes de Elasmotherii reliquiis.

$4^{\circ}$. St. Petersburg, 1864.

BRANDT (J. F.). Ueber die Classification der kaltblütigen Rückenmarkthiere. $4^{\circ}$. St. Petersburg, 1865.

BRANDT (J. F.). Ueber die Gattung der Klippschliefer (Hyrax, Herm.). $\quad 4^{\circ}$. St. Petersburg, 1869.

BRANDT (J. F.). De Dinotheriorum genere Elephantidorum.

$4^{\circ}$. St. Petersburg, 1869.

BRANDT (J. F.). Beiträge zur Naturgeschichte des Elens. $4^{\circ}$. St. Petersburg, 1870.

BRANDT (J. F.). Untersuchungen über die fossilen und subfossilen Cetaceen Europa's. $4^{\circ}$. St. Petersburg, 1873.

BRANDT (J. F.). Ergänzungen zu den fossilen Cetaceen Europa's.

$4^{\circ}$. St. P'etersburg, 1874.

BRANDT (J. F.). Versuch einer Monographie der Tichorhinen Nashörner nebst Bemerkungen über Rhinoceros leptorhinus, Cuv.

$4^{\circ}$. St. Petersburg, 1877.

BRANDT (J. F.). Mittheilungen über die Gattung Elasmotherium.

$4^{\circ}$. St. Petersburg, 1878.

BRANDT (J. F.). Tentamen synopseos Rhinocerotidum viventium et fossilium. $\quad 4^{\circ}$. St. Petersburg, 1878. 
BRANDT (J. F.) \& RATZEBURG (J. T. C.). Getreue Darstellung und Beschreibung der Thiere die in der Arzneimittellehre in Betracht Kommen.

40. Berlin, 1829-33.

BRANTS (A.). De Tardigradis.

$4^{\circ}$. Lugduni Batavorum, 1828.

BRASS (A.). Biologische Studien. I. Theil. Die Organisation der thierischen Zelle.

8. Halle, 1883.

BRACER (F.). Beschreibung und Beobachtung der österreichischen Arten der Gattung Chrysopa.

$4^{\circ}$. Wien, 1850.

BRAUER (F.). See 'Notara.'

BRAUER (F.) \& LOEW (F.). Neuroptera Austriaca.

8․ Wien, 1857.

BRAUN (Max). Dio thierischen Parasiten des Menschen. $8^{\circ}$. Würtzburg, 1883.

BRAUN (Max). Zur Entwicklungsgeschichte des breiten Bandwurmes.

8. . Wïrtzburg, 1883.

BRAdNe (W.). See Pertodicals : Leipzig.

BREE (C. R.). A History of the Birds of Europe not observed in the British Isles.

4 vols. $8^{\circ}$. London, 1863-67. 2nd edition. 5 vols. $8^{\circ}$. London, 1875-76.

BREHM (A. E.). Ergebnisse einer Reise nach Habesch. 8. Hamburg, 1863.

BREHM (A. E.) \& ROSSMÄSSLER (E. A.). Die Thiere des Waldes. 2 vols. $8^{\circ}$. Leipzig und Heidelberg, 1864-67.

BREHMI (A. E.) \& ZIMMERMANN (T. F.). Bilder und Skizzen aus dem zoologischen Garten zu Hamburg.

8. Hamburg, 1865.

BREHII (C. L..). Handbuch der Naturgeschichte aller Vögel Deutschlands.

8.. Ilmenau, 1831. 
BREHM (C. L.). Der vollständige Vögelfang.

8․ Weimar, 1855.

BREHM (C. L.). See Jones (T. R.). Book of Birds.

BREHII (J. E.). Thierleben. I.-X. 8o. Leipzig, 1880.

BREHM (L.) \& PAESSLER (W.). Die Eier der Europäischen Vögel gemalt von T. W. J. BAEDEKER.

4 vols. in 2. fol. Leipzig \& Iserlohn, 1855.

BRÊME (Le Mr. F. de). Essai monographique des Cossyphides.

8o. Paris, 1842.

BREMER (O.). Lepidopteren Ost-Sibiriens, insbesondere des Amur-Landes.

$4^{\circ}$. St. Petersburg, 1864.

BREMER (O.) \& GREY (W.). Beiträge zur Schmetterlings-Fauna des Noerdlichen China's.

8. St. Petersburg \& Helsingfors, 1853.

BREMI-WOLF (J. J.). Catalog der Schweizerischen Coleopteren. 8. . Zurich, 18506.

BREMSER (J. G.). Ueber lebende Würmer im lebenden Menschen. Nebst einem Anhange über Pseudo-Helminthen. $4^{\circ}$. Wien, 1819.

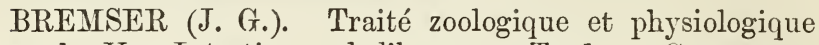
sur les Vers Intestinaux de l'homme. Trad. par Grundeter ; revu par De Blainville.

Texte, $8^{\circ}$. Planches, 4\%. Paris, 1828-37.

BRENCHLEY (J. L.). See ' Curaçoa.'

BRESCHET (G.). Histoire anatomique et physiologique d'un organe vasculaire dans les Cétacés. $4^{\circ}$. Paris, 1836.

BRESCHET (G.). Recherches anatomiques et physiologiques sur l'organe de l'Ouie et l'Audition.

2nd edition. $4^{\circ}$. Paris, 1836. 
BRESCHET (G.). Recherches anatomiques et physiologiques sur l'Organe de l'Ouie des Poissons.

$4^{\circ}$. Paris, 1838.

BRETON (W. H.). Scandinavian Sketches.

8. London, 1835.

BREWER (T. M.). See Baird (S. F.).

BRISCHKE (C. G. A.). Abbildungen und Beschreibungen der Blattwespen-Larven. Vorwort von J. T. C. RatzeBURg. $4^{\circ}$. Berlin, 185 อ̃.

BRISOUT DE BARNETILLE (C.). See WeNcker (J.).

BRISSON (A. D.). Regnum Animale in Classes IX. distributum sive Synopsis Methodica.

8. Lugduni Batavorum, 1762.

BRISSON (A. D.). Ornithologia, sive Synopsis methodica Avium.

6 vols. $4^{\circ}$. Paris, 1760.

Another edition.

2 vols. in 1. 80. Lugduni Batavorum, 1763.

BRITISH ORNITHOLOGISTS' UNION. A List of British Birds compiled by a Committee of the B. O. U.

8. London, 1883.

BROCA (P.). Mémoires d'Anthropologie.

Vols. I.-IV. $8^{\circ}$. Paris, 1871-83.

BROCKMANN (H.). De Pancreate Piscium.

$4^{\circ}$. Rostochii, 1846.

BRODERIP (W. J.). Zoological Recreations.

8. London, 1847.

BRODERIP (W. J.). Leaves from the Note-book of a Naturalist.

8. London, 1852.

BRODIE (P. B.). A History of the Fossil Insects in the Secondary Rocks of England.

8. London, 1845.

BROGIANI (D.). De Veneno Animantium naturali et adquisito tractatus.

$8^{\circ}$. Florentice, 1755. 
BRONGNIART (C. J. E.). Annexe au "Species des Hyménoptères d'Europe" par M. E. André. Les Hyménoptères Fossiles.

8.. Paris, 1881.

BRONN (H. G.). Der Schädel des Hatitherium schinzi, Kaup. $8^{\circ}$. Stuttgart, 1862.

BRONN (H. G.). Die Klassen und Ordnungen des Thier-Reichs. roy. $8^{\circ}$. Leipzig und Heidelberg, 1859-83. [In progress.]

BRONN (H. G.). Index Palæontologicus.

3 vols. $8^{\circ}$. Stuttgart, 1848-49.

BROOKES (S.). An Introduction to the Study of Conchology.

$4^{\circ}$. London, 1815.

BROOKS (W. K.). Chesapeake Zoological Laboratory. Scientinic Results, 1878.

$8^{\circ}$. Baltimore, 1879.

BROOKS (W. K.). Handbook of Invertebrate Zoology.

8. Boston, 1882.

BROT (A.). Étude sur la Famille des Naiades.

8\%. Bâle et Genève, 1867.

BROT (A.). Matériaux pour servir à l'Étude de la famille des Mélaniens. Parts I.-III. 8o. Paris, 1862-72.

BROUSSONET (P. M. A.). Ichthyologia sistens Piscium descriptiones et icones.

$4^{\circ}$. Londini, 1782.

BROWN (J. A. H.), CORDEAUX (J.), KERMODE (P. M. C.), BARRINGTON (R. M.), and MORE (A. G.). Reports on the Migration of Birds. $8^{\circ}$. London, 1880-83.

BROWN (P.). New Illustrations of Zoology.

$4^{\circ}$. London, 1776.

BROWN (R.). The Races of Mankind. 4 vols. in $2.4^{\circ} . \quad$ London, n. d.

BROWN (T.). The Book of Butterflies, Sphinxes, and Moths. [Vols. 75 and 76 of Constable's Miscellany.]

2 vols. $16^{\circ}$. London, 1832. 
BROWN (T.). Illustrations of the Recent Conchology of Great Britain and Ireland. 2nd edit. 4\%. London, 1844.

BROWN (W.). The Natural History of the Salmon.

$12^{\circ}$. Glasgow, 1862.

BROWNE (P. A.). Trichologia Mammalium.

$4^{\circ}$. Philadelphia, 1853.

BRUAND (Th.). Catalogue systématique et synonymique des Lépidoptères du département du Doubs.

roy. $8^{\circ}$. Besançon, 1845.

BRUCH (C.). Vergleichende Osteologie des Rheinlachses. fol. Mainz, 1861.

BRUCH (C.). Vergleichende Osteologie des Rheinlachses. fol. Mainz, 1875.

BRUCH (C.). See Periodicals : Frankfurt a. Main.

BRÜCKMANN (F. E.). Bibliotheca Animalis : oder Verzeichniss der meisten Schriften so von Thieren und deren Theilen handeln.

$12^{\circ}$. Wolffenbüttel, 1743 .

BRÜHL (C. B.). Anfangsgründe der vergleichenden Anatomie aller Thierklassen.

Text, $8^{\circ}$. Atlas, $4^{\circ}$. Wien, 1847.

BRÜHL (C. B.). Kleine Beiträge zur Anatomie der Haussäugethiere.

fol. Wien, 1850.

BRÜHL (C. B.). Osteologisches aus dem Pariser Pflanzengarten.

$4^{\circ}$. Wien \& Leipzig, 1856.

BRÜHL (C. B.). Zur Kenntniss des Orangkopfes und der Orangarten.

$4^{\circ}$. Wien, 1856.

BRÜHL (C. B.). Das Skelet der Krokodilinen.

$4^{\circ}$. Wien, 1862.

BRÜHL (C. B.). Zootomie aller Thierklassen.

Band I. Lief. 1-30. $4^{\circ}$. Wien, 1877-83. 
BRULle (A.). See De Laporte, F. L. Comte de Castelinad.

BRÜMMER (J.). Anatomische und histologische Untersuchungen iiber den zusammengesetzten Magen verschiedener Säugethiere.

8. Leipzig, 1876.

BRUNNER (C. von W.). Monographie der Phaneropteriden.

$8^{\circ}$. Wien, 1878.

BRUNNER (C. von W.). Nonveau Système des Blattaires. 8. Vienna, 1865.

BRUNNER (C. von W.). Prodromus der Europäischen Orthopteren.

8․ Leipzig, 1882.

BRÜNNICH (M. T.). Eder Fuglens Beskrivelse.

12. Kiobenhavn, 1763 .

BRÜNNICH (M. T.). Die natürliche Historie des EiderVogels.

$12^{\circ}$. Kopenhagen, $\mathbf{I 7 6 3 .}$

BRÜNNICH (M. T.). Ornithologia Borealis.

8. Hafinice, 1764.

BRÜNNICH (M. T.). Entomologia sistens Insectorum tabulas systematicas.

8. Kioberhavn, 1764.

BRÜNNICH (M. T.). Spicilegia Zoologica. MS. 1765.

BRÜNNICH (M. T.). Ichthyologia Massiliensis.

8. Hafnice \& Lipsice, 1768.

BRÜNNICH (M. T.). Zoologiæ fundamenta.

8. Hafnice \& Lipsice, 1772.

BRUNS (V.). De Nervis Cetaceorum Cerebralibus.

8․ Tubingoe, 1836.

BRUNTON (T. L.). See Krein (E.).

BRUSINA (S.). Ipsa Chiereghinii Conchylia.

8. Pisct, 1870. 
BRU'TZER (G. G.). De Scaphirhyncho Rafinescii disquisitiones anatomicæ.

8. Dorpati Livono'um, 1859.

BUCH (L.). Ueber Productus oder Leptcena.

$4^{\circ}$. Berlin, 1842.

BUCH (L.). Ueber Cystideen.

4\%. Berlin, $18 \pm 5$.

BUCH (L.). Ueber Ceratiten.

4\%. Berlin, 1849.

BUCHANAN (F. H.). An account of the Fishes found in the River Ganges and its Branches.

$4^{\circ}$. Edinburgh, 1822.

BUCHECKER (H.). Systema Entomologiæ.

8. München, 1876.

BUCKMAN (J.). See Cambridge (O. P.).

BUCKTON (G. B.). A Monograph of the British Aphides.

See RAY Soctety.

3 vols. $8^{\circ}$. London, 1876-81.

BUFFON (G. L. Le Clerc, Comte de). Histoire naturelle. 33 vols. $4^{\circ}$. Paris, 1749-89.

BUFFON (G. L. Le Clerc, Comte de). Histoire Naturelle des Oiseaux. Planches enluminées.

10 vols. fol. Paris, 1770-86.

BUFFON (G. L. Le Clerc, Comte de). CEurres Complètes. Par M. Le CoMte de Lacepk̀de.

31 vols. $8^{\circ}$. Paris, 1825.

BUFFON (G. L. Le Clerc, Comte de). CEurres complètes. Annotées par M. Flodrens. 12 vols. 8․ Paris, 1852-54.

BUFFON (G. L. Le Clere, Comte de). See Lesson (R. P.).

BUfFON (G. L. Le Clere, Comte de). See Trgny (F. M. G. T. de).

BUFFON (H. N. de). Buffon, sa famille, ses collaborateurs, et ses familiers.

$8^{\circ}$. Paris, 1863. 
BUHLE (C. A.). Die Tag- und Abend-Schmetterlinge Europa's. 4. Leipzig, 1837.

BUHLE (C. A.). See Nadmann (J. F.).

'BULLDOG.' The North Atlantic Sea-bed: a Diary of the Toyage of H.M.S. 'Bulldog' in 1860. By G. C. Wallich. Part I. (all published). $4^{\circ}$. London, 1862.

BULLER (W. L.). Essay on the Ornithology of New Zealand. 8०. Dunedin, 1865.

BULLER (W. L.). History of the Birds of New Zealand. $4^{\circ}$. London, 1873.

BULLER (W. L.). Manual of the Birds of New Zealand. $8^{\circ}$. Wellington, N. Z., 1882.

BUNGE (A.). Untersuchungen zur Entwickelungsgesthichte des Beckengürtels der Amphibien, Reptilien und Vögel.

$8^{\circ}$. Dorpat, 1880.

BURCHELL (J.). A List of Quadrupeds from Southern Africa. 8.. London, 1817.

BURDACH (K. F.). Der Mensch nach den verschiedenen Seiten seiner Natur.

$8^{\circ}$. Stuttgart, 1837.

BURDON-SANDERSON (J.). See KLeIN (E.).

BURGESS (E.). See Henz (N. M.).

BURMEISTER (H.). De Insectorum systemate naturali. 80. Halis Saxonum, 1829.

BURMEISTER (H.). Lehrbuch der Naturgeschichte.

80. Halle, 1830.

BURMEISTER (H.). Handbuch der Entomologie. 5 vols. $8^{\circ}$. Berlin, 1832-47.

BURMEISTER (H.). Beiträge zur Naturgeschichte der Rankenfüsser (Cirripectia). 4. Berlin, 1834. 
BURMEISTER (H.). Zur Naturgeschichte der Gattung Calandra. $4^{\circ}$. Berlin, 1837.

BURMEISTER (H.). Handbuch der Naturgeschichte.

8. Berlin, 1837.

BURMEISTER (H.). Genera quædam Insectorum.

8. Berolini, 1838-46.

BURMEISTER (H.). Beiträge zur näheren Kenntniss der Gattung Tarsius.

4\%. Berlin, 1846.

BURJEISTER (H.). Athlophorus Klugii.

$4^{\circ}$. Halle, 1847.

BURMEISTER (H.). Verzeichniss der im zoologischen Museum der Universität Halle-Wittenberg aufgestellten Säugethiere, Vögel, und Amphibien. $\quad 8^{\circ}$. Halle, 1850.

BURMEISTER (H.). Systematische Uebersicht der Thiere Brasiliens. 8.. Berlin, 1854-56.

BURMEISTER (H.). Erläuterungen zur Fauna Brasiliens. fol. Berlin, 1856.

BURMEISTER (H.). Zoonomische Briefe. Allgemeine Darstellung der thierischen Organisation.

2 vols. in $1.8^{\circ}$. Leipzig, 1856.

BURMEISTER (H.). Systematische Uebersicht der Sphingidæ Brasiliens.

$4^{\circ}$. Halle, 1856.

BURMEISTER (HI.)。 Reise durch die La Plata-Staaten. 2 vols. 8o. Halle, 1861.

BURMEISTER (H.). Ein neuer Chlamyphorus. $4^{\circ}$. Halle, 1863.

BURMEISTER (H.). Description physique de la République Argentine. Vols. I., II., III., \& V.

Text, roy. 8\%. Paris \& Buenos Ayres, 1876-79. Atlas. fol. \& $4^{\circ}$. Buenos Ayres, 1879-81.

BURMEISTER (H.). Bericht über diø Feier des 50 jährigen Doctor Jubilaeums \&c. $\quad 8^{\circ}$. Buenos Ayres, 1880. 
BURMeisteR (H.). See Nitzsch (C. L.).

BURjeister (H.). See Periodicals: Buexos Arres.

BURROW (E. J.). Elements of Conchology, according to the Linnæan System.

8. London, 1815. New edition. $8^{\circ}$. London, 1825.

BURTON (R. F.). Falconry in the Valley of the Indus. 8.. London, 1852.

BURY (Mrs.). Figures of remarkable Forms of Polycystins, or allied organisms, in the Barbados Chalk Deposit.

$4^{\circ}$. London, 1860-61.

BUSCH (F. G. J.). Ad Anatomiam nonnullorum Heliceorum Agri Bonnensis Symbolæ.

$8^{\circ}$. Bonnce, 1855.

BCSCH (G.). De Selachiorum et Ganoideorum encephalo. $4^{\circ}$. Berolini, 1848.

BUSHMAN (An Old). See WhEelwright (H.).

BUSK (G.). On the Alternation of Generations.

8.. London, 1845.

BUSK (G.). See Musedm (British).

BUSK (G.), 'IULK (A.), and HALIIDAY (A. H.). Reports on Zoology for 1843-44. Translated from the German.

See Ray Societr.

$8^{\circ}$. London, 1847.

BUSQUET (L.). Manuel de Hirudiculture, ou de L'Elève des Sangsues.

$8^{\circ}$. Bordecuux, 1854.

BUTLER (A. G.). Catalogue of Diurnal Lepidoptera of the family Satyridae in the Collection of the British Museum.

See Museum (British).

8. . London, 1868.

BUTLER (A. G.). Catalogue of Diurnal Lepidoptera described by Fabricius in the Collection of the British Museum.

See Musedul (British).

8․ London, 1869. 
BUTLER (A. G.). Lepidoptera Exotica.

$4^{\circ}$. London, 1869-74.

BUTLER (A. G.). Illustrations of Typical Specimens of Lepidoptera Heterocera in the Collection of the British Museum. Parts 1, 2, 3, 5. 4\% . Lonlon, 1877-78-79-81.

See Walsingham.

[In progress.]

BUTLER (A. G.). On the Butterflies of New Zealand. With Preface by J. D. Evrs. $\quad 8^{\circ}$. Christchurch, 1880.

BUtler (A. G.). See 'Erebts' asd 'Terror.'

BÜTSCHLI (O.). See Bronn (H. G.).

C.

CABANIS (J.). Museum Heineanum.

2 vols. $8^{\circ}$. Halberstadt, 1850-63.

CABANis (J.). See Periodicals : Berlin, Cassel, and LEIPZIG.

CABANIS (J.). See Von Der Decken's Reisen.

CABANIS (J.) und REICHENOW (A.). See Periodicals : BerLin. Ornithologisches Centralblatt.

CABOT (L.). The Immature State of the Odonata. Part I. Gomphina. Part II. Asschina.

40. Cambridge, Mass., 1872-81.

See Museum: Harvard College. Memoirs.

CAILLIAUD (F.). Catalogue des Radiaires, des Annélides, des Cirrhipèdes et des Mollusques de la Loire-Inférieure.

8. Nantes, 1865.

CALISCH (J. M.). See Dictionaries: Dutch-Evglish.

CALONNE (De). Museum Calonnianum.

8. London, 1797. 
CAIORI (L.). Sull' Anatomia dell' Axolotl.

$4^{\circ}$. Bologna, 1852.

CALORI (L.). Della Stirpe che ha popolata l'antica necropoli alla certosa di Bologna. $\quad 4^{0} . \quad B o l o g n a, 1873$.

CALVI (G.). Catalogo d' Ornitologia di Genova.

8.. Genova, 1828.

CALWER (C. G.). Naturgeschichte der Käfer Europa's.

$8^{\circ}$. Stuttgart, 1858.

CAMBRIDGE (O. P.). The Spiders of Dorset. Edited by J. BUCKMAN.

2 vols. $8^{\circ}$. Sherboine, 1879.

CAMERANO (L.). Gli Insetti ; introduzione allo studio dell' Entomologia.

8. Torino e Roma, 1879.

CAMERANO (L.) \& LESSONA (M.). Biblioteca della Zoologia e Anatomia comparata ín Italia.

8. Torino, 1878-80.

CAMPBELL (J.). A treatise of modern Faulconry.

8. Edinburgh, 1773.

CAMPER (P.). Différence des traits du Visage. Traduit par D. B. Q. D'IsJonval.

$4^{\circ}$. Autrecht, 1791.

CAMPER (P.). Naturgeschichte des Orang-Utang und einiger andern Affenarten. Des Africanischen Nashorns und des Rennthiers. Ed. J. F. Herbelz.

$4^{\circ}$. Dusseldorf, 1791.

CAMPER (P.). Description anatomique d'un Eléphant mâle.

fol. Paris, 1802.

CAMIPER (P.). Euvres, qui ont pour objet l'histoire naturelle, la physiologie et l'anatomie comparée.

3 vols. $8^{\circ} \& 1$ vol. fol. Paris, 1803.

CAMPER (P.). Observations anatomiques sur la structure intérieure et le squelette des plusieurs espèces de Cétacées. $4^{\circ} \&$ fol. Paris, 1820. 
CAMPER (P. \& A. G.). Recueil de Planches pour servir aux observations anatomiques sur la structure intérieure et le squelette de plusieurs espèces de Cétacés.

fol. Paris, 1820.

CANDĖZE (E.). Monographie des Elatérides.

4 vols. $8^{\circ}$. Liège, 1857-63.

CANDEZE (E.). Révision de la Monographio des Elatérides. 8.. Bruxelles, 1874.

CANDOLLE (A. de). Histoire des Sciences.

8. Genève, Bâle, \& Lyon, 1873.

CANEFRI (C. T.). Glanures dans la Faune Malacologique de l'Ile Maurice. 80. Bruxelles, 1881.

CANESTRINI (G.). See Periodicals: Genoa and Modena. Archivio per la Zoologia.

CANESTRINI (G.). See Fauna d' Italia.

CANESTRINI (G. \& R.). I Gamasi Italiani. Monografia.

8. Padova, 1882.

CANTENER (L. P.). Catalogue des Lépidoptères du Département du Var. 80. Paris, 1833.

CANTOR (T.). Zoology of Chusan. $\quad 4^{\circ} . \quad$ Chusan, 1840.

CANTOR (T.). Catalogue of Reptiles and Fishes of the Malayan Peninsula. 8. Calcutta, 1847-50.

CANTRATNE (F.). Malacol ogie Méditerranéenne. 4. Bruxelles, 1841.

CAPEL (C. C.). Trout Culture.

8. London, 1877.

CAPELLINI (G.). Della Balena di Taranto.

$4^{\circ}$. Bologna, 1877.

CAPELLINI (G.). Sulla Balenothera di Mondini.

$4^{\circ}$. Bologna, 1877. 
CAPPER (J.). Journal of the Ceylon Branch of the Royal Asiatic Society.

$8^{\circ}$. Colombo, 1856-58.

CARA (G.). Ornitologia Sarda.

8. Torino, 1842.

CARBONNIER (P.). Guide pratique du Pisciculteur.

8. Paris, 1864.

CARBONNIER (P.). L'Ecrevisse.

8. $\quad$ Paris, ${ }^{-1869 .}$

CARCASSONNE (MI.). See Farines (MI.).

CARLEER (I. H. M ). Examen des principales Classifieations adoptées par les zoologistes. $8^{\circ}$. Bruxelles, 1861.

CARLIER (A. A.). Anatomie Philosophique.

8.. Puris, 1883.

CARMINATI (B.). De animalium ex mephitibus et noxiis halitibus interitu, ejusque propioribus causis.

$4^{\circ}$. Laude Pompeja, 1777.

Carpenter (P. H.). See Musedm: Harvard College.

CARPENTER (P. P.). Report on the Mollusca of the West Coast of North America. $\quad 8^{\circ}$. London, 1857.

CARPENTER (P. P.). Catalogue of Mazatlan Shells.

120. London, 1857.

CARPENTER (P. P.). Lectures on Mollusca.

8. Washington, 1861.

CARPENTER (P. P.). The Mollusks of Western North America.

$8^{\circ}$. Washington, 1872 .

CARPENTER (P. P.). See Suithsonian Miscellaneods Collections.

CARPENTER (W. B.). Shell.

(Ex. Cycl. Anat. \& Phys.) $\quad 8^{\circ}$. London, n. d.

CARPENTER (W. B.). Physiological Inferences deduced from the Structure of the Nerrous System in the Invertebrata.

8. . Edinburgh, 1839. 
Carpenter (W. B.). See 'Challenger.'

CARPENTER (W. B.), PARKER (W. K.), and JONES (T. R.). Introduction to the Study of the Foraminifera.

See $\mathrm{R}_{\mathrm{A}} \mathrm{Y}$ Sochetr. fol. London, 1862.

CARPENTER (W. L.). See Survers and Explorations: WASHINGTON.

CARRIERE (J.). Studien über die Regenerations-Erscheinungen bei den Wirbellosen. I. Pulmonaten.

$4^{\circ}$. Würzburg, 1880.

CARringtoN (J. T.). See Periodicals: London.

CARRUCCIO (A.). Catalogo Metodico degli Animali riportati dalle escursioni nelle Provincie meridionali, in Sicilia e in Sardegna dal Prof. A. Targioni-Tozzetti. Part 1. Vertebrati.

8. Milano, 1870.

CARTER (H. J.). Geology of the Island of Bombay. roy. $8^{\circ}$. Calcutta, 1850 .

CARUS (C. G.). Lehrbuch der Zootomio.

Text, 8‥ Atlas, $4^{\circ}$. Leipzig, 1818.

CARUS (C. G.). Erläuterungstafeln zur vergleichenden Anatomie.

fol. Leipzig, 1826.

CARUS (C. G.). Lehrbuch der rergleichenden Zootomie. Text \& Atlas. 8. Leipzig, 1834.

CARUS (C. G.). Neuer Atlas der Cranioskopie.

$4^{\circ}$. Leipzig, 1864.

CARUS (J. V.). Zur Kenntniss des Generationswechsels.

8. Leipzig, 1849.

CARUS (J. V.). Icones Zootomicæ. fol. Leipzig, 1857.

CARUS (J. V.). Ueber die Werthbestimmung zoologischer Merkmale.

4. Leipzig, 1854. 
CARUS (J. V.). Ueber die Leptocephaliden.

$4^{\circ}$. Leipzig, 1861.

CARUS (J.V.). See Periodicals : Leipzig. Zoologischer Anzeiger.

CARUS (J. V.) et ENGELMANN (W.). Bibliotheca Zoologica. 2 vols. $8^{\circ}$. Leipzig, 1861.

CARUS (J. V.) und GERSTAECKER (C. E. A.). Handbuch der Zoologie.

80. Leipzig, 1868-75.

CASsElL. See Duncan (P. M.).

CASSELL. See Jones (T. R.). Book of Birds.

CASSERIUS (J.). De Vocis Auditusque Organis Historia Anatomica.

fol. Ferrarice, 1601.

CASSIN (J.). Illustrations of the Birds of California, Texas, \&c. roy. $8^{\circ}$. Philadelphia, 1862.

CASSIN (J.). See Surveys and Explorations: U.S., Philladelphia.

CASTELLI (P.). Hyana odorifera. $\quad 4^{\circ} . \quad$ Messance, 1638.

CASTELNAU (F. L. Comte de). Mémoire sur les Poissons de l'Afrique Australe. $\quad$ 8. Paris, 1861.

Castelnau (F. L. Comte de). See De Laporte, F. L. Comte de Castelajad.

CATALOGUS Coleopterorum Europæ. 8 $^{\circ}$. Stettin, 1855.

CATESBY (M.) and EDWARDS (G.). See Seligmany (J. M.).

CATLIN (G.). Letters and Notes on the Manners, Customs, and Condition of the North American Indians. 3rd edition. 2 vols. $8^{\circ}$. London, 1842. 
CATLOW (A.) and REEVE (L.). The Conchologist's Nomenclator. $\quad 8^{\circ}$. London, 1845.

CATLOW (M. E.) Popular Scripture Zoology.

sm. $4^{\circ}$. London, 1852.

CATLOW (M. E.). Popular British Entomology. 2nd edition. sm. $4^{\circ}$. London, 1852.

CATON (J. D.). American Cervus. $\quad 8^{\circ}$. Ottawa, 1868.

CATON (J. D.). The Antelope and Deer of America.

8. New York, 1877.

CA'TTANEO (G.). Le Colonie Lineari e la Morfologia dei Molluschi.

$8^{\circ}$. Milano, 1883.

CATTIE (J. T.). Vergelijkend-Anatomische en Histologische Onderzoekingen van de Epiphysis Cerebri der Plagiostomi, Ganoidei en Teleostei. $\quad 8^{\circ}$. Leiden, 1881.

CAUVIN (C.). Mémoire sur les Races de l'Océanie.

8. Paris, 1882.

CAVANNA (G.). Elementi per una Bibliografia Italiana, intorno all' idrofauna agli allevamenti degli Animali Acquatici $\theta$ alla Pesca. $\quad 8^{\circ}$. Firenze, 1880.

CAVOLINI (P.). Abhandlungen ïber Pflanzen-Thiere des Mittelmeers. Translated from the Italian by WILHELM Sprengel. Edited by Kurt Sprengel.

4․ Nürnberg, 1813.

CEDERHIELM (J.). Faunæ Ingricæ Prodromus. 8. Lipsia, 1798

CETTI (F.). Storia naturale di Sardegna.

3 vols. $8^{\circ}$. Sassari, 1774-77.

CHABRIER (J.). Essai sur le Vol des Insectes. 40. Paris, 1822. 
CHALLENGER. Reports on the Scientific Results of the Voyage of H.M.S. 'Challenger' during the years 1873-76, under the command of Capt. Nares and Capt. Thomson. Prepared under the superintendence of Sir C. WrviLte Thouson, Knt., F.R.S. $\quad 4^{\circ}$. London, 1880-83.

Brachiopoda. By T. Davidson.

Pennatulida. By A. V. KöLliker.

Ostracoda. By G. S. Brady.

Bones of Cetacea. By W. Turner.

Development of the Turtle. By W. K. Parker.

Shore Fishes. By A. Günther.

Corals. By H. N. MoseLey.

Birds. By the Marquis of Tweeddale, P. L. Sclater, O. Finsch, T. Saltadori, W. A. Forbes, H. Saunders, O. Salvin, and A. H. Garrod.

Echinoidea. By A. Agassiz.

Pycnogonida. By P. P. C. Hoek.

Anatomy of the Petrels. By W. A. Forbes.

Deep-Sea Medusæ. By E. HaEckeL.

Holothuroidea. By H. TвÉEL.

Ophiuroidea. By T. LyMan.

Marsupialia. By D. J. Cunningham.

Actiniaria. By R. Hertwig.

Tunicata. By W. A. Herdman.

Spheniscidæ. By M. Watson.

Pelagic Hemiptera. By T. B. Whire.

Orbitolites. By W. B. Carpenter.

Hydroida. By G. J. Allman.

[In progress.]

CHAMBERS (C. H.). See Gastaldi (B.).

CHAPUIS (F.). Monographie des Platypèdes.

8. Liége, 1865.

CHAPUIS (F.). Le Pigeon Voyageur.

$12^{\circ}$. Verviers, 1876.

CHARAS (M.). Nouvelles Expériences sur la Vipere. 2nd edition. $8^{\circ}$. Paris, 1694.

CHARLES IX. La Chasse Royale. (Ed. Chevrect.) $12^{\circ}$. Paris, 1858.

CHARLESWORTH (E). See Periodicals: London. 
CHARLETON (G.). Onomasticon zoicon.

$4^{\circ}$. Londini, 1668.

2nd edition. $4^{\circ}$. Londini, 1671.

CHARLETON (G.). Exercitationes de differentiis et nominibus Animalium. $\quad 4^{\circ}$. Oxonice, 1677.

CHARPENTIER (A.). Monographie des Sangsues médicinales et officinales.

80. Paris, 1838.

CHARPENTIER (T.). Horæ entomologicæ.

$4^{\circ}$. Wratislavice, 1825.

CHARPENTIER (T.). Libellulinæ Europææ.

$4^{\circ}$. Lipsice, 1840.

CHARPENTIER (T.). Orthoptera descripta et depicta.

$4^{\circ}$. Lipsice, 1841-45.

CHARPENTIER (T.). Die Zinsler, Wickler, Schaben und Geistchen des systematischen Verzeichnisses der Schmetterlinge der Wiener Gegend.

$8^{\circ}$. Braunschweig, 1821.

CHARPENTIER (T.). Verzeichniss der Schmetterlinge der Wiener Gegend, mit Anmerkungen von J. ZINCKEN.

$8^{\circ}$. Braunschweig, 1821.

CHATIN (J.). La Trichine et la Trichinose.

8․ Paris, 1883.

CHAUDOIR (M. de) \& HOCHHU'TH(H.). Enumeration des Carabiques et Hydrocanthares du Caucase.

8.. Kiew, 1846.

CHAUVEAU (A.). Traité d'Anatomie Comparée des Animaux domestiques. (Ed. Arlong.) $8^{\circ}$. Paris, 1879.

CHEMNITZ (J. H.). See Martini and Chemnitz.

CHENU (J.C.). Leçons élémentaires d'Histoire Naturelle. 8. Paris, 1847.

CHENU (J. C.). Encyclopédie d'histoire naturelle.

4.. Paris, 1855-80. 
CHENU (J. C.). Manuel de Conchyliologie et de Paléontologie Conchyliologique. 2 vols. in 1. $8^{\circ}$. Paris, 1859-62.

CHESAPEAKE 7oological Laboratory. See BrooKs (W.K.).

CHESELDEN (W.). Osteographia, or the Anatomy of the Bones. fol. London, 1733.

CHEVRIER (F.). Description des Chrysides du Bassin du Léman. 8.. Paris, 1862.

CHEVRIER (F.). Essai Monographique sur les Nysson du Bassin du Léman (Insectes Hyménoptères).

$8^{\circ}$ Bale \& Genève, 1867.

CHEvrolat (A.). Coléoptères du Mexique.

$12^{\circ}$. Strasbourg, 1834.

CHIAJE (S. delle). Memorie sulla storia e anotomia degli Animali senza rertebre del regno di Napoli.

5 vols. $4^{\circ}$. Napoli, 1823-29.

CHIAJE (S. delle). Compendio di Elmintografia Umana. 80. Napoli, 1833.

CHIAJE (S. delle). Descrizione e anotomia degli Animali Invertebrati della Sicilia Citeriore.

8 vols. in 2. fol. N'apoli, 1841-44.

CHIEREGHINI (S.). See Brtsina (S.).

CHILDREN (J. G.). Translation of Lamarck's ' Genera of Shells.' See LaMarcK (J. B.).

CHRISTEN (C.). De Lama Observationes nonnullas Anatomicas.

$8^{\circ}$. Tüibingo, 1827.

CHUN (C.). See FaUNa UNd Flora des Golfes von Neapel.

CHURCH (J.). Cabinet of Quadrupeds.

2 vols. $4^{\circ}$. London, 1805.

CLAIRVILLE (J. de). Entomologie Helretique.

8․ Zurich, 1798. 
CLAPAREDE (A. R. E.). Beobachtungen über Anatomio und Entwicklungsgeschichte Wirbelloser Thiere.

$4^{\circ}$. Leipzig, 1863.

CLAPAREDE (E.). De la Formation et de la Fécondation des Eufs chez les Vers Nématodes.

4. Genève, 1859.

CLAPAREDE (E.). Recherches anatomiques sur les Oligochètes.

$4^{\circ}$. Genève, 1862.

CLAPAREDE (E.). Glanures zootomiques parmi les Annélides de Port-Vendres (Pyrénées). $\quad 4^{\circ}$. Genève, 1864.

CLAPAREDE (E.). Les Annélides Chétopodes du Golfe de Naples.

4. Genève et Bâle, 1868.

CLAPAREDE (E.). Recherches sur la structure des Anvélides sédentaires.

$4^{\circ}$. Genève, 1873.

CLARK (H.). Catalogue of Halticidae. 80. London, 1860. See Museum (British).

CLARK (H.). A Catalogue of Phytophaga. [Coleoptera, Pseudotetramera.]

8.. London, 1866.

CLARK (H.). Letters Home.

8. London, 1867.

CLARK (W.). A History of the British Marine Testaceous Mollusca.

8. London, 1855.

CLARKE (R.). Sierra Leone.

8. London, 1843.

CLARKE (W. B.). Notice of a Species of Seal apparently new to the British Shores.

$4^{\circ}$. Ipswich, 1847.

CLARKE (W. B.). See NUNN (J.).

CLARKE (W. E.) \& ROEBUCK (W. D.). Handbook of the Yorkshire Vertebrata. 8. London, 1881.

CLAUDIUS (M.). Physiologische Bemerkungen über das Gehörorgan dor Cetaceen und das Labyrinth der Säugethiere.

$8^{\circ}$. Kiel, 1858. 
CLAUDIUS (M.). Das Gehörorgan von Rhytina Stelleri. $4^{\circ}$. St. Petersburg, 1867.

CLAUS (C.). Die freilebenden Copepoden der Fauna Deutschlands. 4º. Leipzig, 1863.

CLACS (C.). Ueber die Grenze des Thierischen und Pflanzlichen Lebens. 4. Leipzig, 1863.

CLAUS (C.). Untersuchungen zur Erforschung der genealogischen Grundlage des Crustaceen-Systems.

4.. Wien, 1876.

CLAUS (C.). Grundzüge der Zoologie.

2 vols. $88^{\circ}$. Marburg, 1880-82.

CLAUS (C.). Untersuchungen über die Organisation und Entwicklung der Medusen. $4^{\circ}$. Prag und Leipzig, 1883.

CLAUS (C.). See Periodicals: Vienna.

CLEMENS (B.). The Tineina of North America. With notes by H. T. Statnton. 8. London, 1872.

CLERCK (C.). Srenska Spindlar. Aranei Suecici. $4^{\circ}$. Stockholmice, 1757.

CLERMONT (Thos. Fortescue, Lord). A Guide to the Quadrupeds and Reptiles of Europe. $8^{\circ}$. London, 1859.

CLESSIN (S.). Deutsche Excursions-Mollusken-Fauna. 8. Nürnberg, 1876-77.

CLOQUET (J.). Anatomie des Vers Intestinaux.

$4^{\circ}$. Paris, 1824.

COBBOLI) (T. Spencer). Entozoa. $\quad 8^{\circ}$. London, 1864. Supplement, 1869.

COBBOLD (T. Spencer). Parasites. A Treatise on the Entozoa of Man and Animals, including some account of the Ectozoa.

8. London, 1879.

COBBOLD (T. Spencer). See Mrsedm: College of SurGEONS. 
COBBOLD (T. Spencer). See Fisheries : London.

COCCHI (J.). Nuova Famiglia di Pesci Labroidi. Monografia dei Pharyngodopilidce.

$4^{\circ}$. Firenze, 1864.

COINDE (J. P.). Description Scientifique de la vie du Myrméléon, sous ses trois états de larve, de nymphe et d'insecto parfait. $16^{\circ}$. Lyon, 1855.

COLlETT (R.). See Surveys \& Exploratrons : Norway.

COLLIE (A.). See 'Blossour.'

COLLIN (J.). See KJ̈̈RböLLING (N.).

COMOLLI (A.). De Coleopteris novis ac rarioribus minusve cognitis provinciae Novocomi.

8.. Ticini-Regii, 1837.

COMSTOCK (J. H.). Report on Insects injurious to Sugar Cane.

$8^{\circ}$. Washington, 1881.

COMSTOCK (J. H.). See REPorts : Washrngton, U.S.

COMTE (A.). Règne Animal disposé en tableaux méthodiques. fol. Paris, 1840. See Cuvier (G.).

CONFIGLIACHI (P.). Sull' Analisi dell' Aria contenuta nella Vesica natatoria dei Pesci.

4. Pavia, 1809.

CONFIGLIACHI (P.) \& RUSCONI (M.). Del Proteo Anguino di Laurenti Monografia.

4\%. Pavia, 1819.

CONRAD (T. A.). Monograph of the Family Unionida, or Naiades of Lamarck. $\quad 8^{\circ}$. Philadelphia, 1835-36.

CONRINGIUS (H.). See Dubravius (J.) \& Heresbach (C.).

CONSTABLE (H.). Miscellany. See Brown (T.).

$67 \quad$ F 2


CONTARINI (N.). Trattato delle Attinie.

$4^{\circ}$. Venezia, 1844.

COOK (A. J.). Manual of the Apiary.

4th edition. $8^{\circ}$. Chicago, 1879.

COOKE (MI.). A treatise on the Insects injurious to fruit and fruit-trees of the State of California.

$8^{\circ}$. Sacramento, 1881.

COOKE (M. C.). Our Reptiles.

$12^{\circ}$. London, 1865 .

COOPER (J. G.). See Strteys and Explorations: U.S., NEw YoRK.

COOTE (H.). The Homologies of the Human Skeleton.

8.. London, 1849.

COPE (E. D.). Check-List of North-American Batrachia and Reptilia.

$8^{\circ}$. Washington, 1875.

COPE (E. D.). See Periodicals : Washivgton.

COPE (E. D.). See Reports : Washington.

COPPINGER (R. W.). See 'AlERT.'

COQUEBERT (A. J.). Illustratio iconographica Insectorum.

4\%. Paris, 1799.

CORBIE. See Boitard (P.).

CORDEAUX (J.). Birds of the Humber District. post 80. London, 1872.

CORDEAUX (J.). See Browr. (J. A. H.) and others.

CORNALIA (E.). Tertebratorum Synopsis in Museo Mediolanense. 4\%. Modoetice, 1849.

CORNALIA (E.). Sulle Branchie transitorie dei Feti Plagiostomi. $4^{\circ}$. Milano, 1857. 
CORNALIA (E.). See Fatna d' Italia.

CORNELIUS (C.). Die Zug- und Wander-Thiere aller Thierklassen.

8. Berlin, 1865.

CORNISH (T.). See FIsHERIES: LoNdoN. International Fisheries Exhibition, 1883.

'CORWIN.' Cruise of the Revenue-Steamer 'Corwin' in Alaska and the N.W. Arctic Ocean in 1881.

Anthropology. By J. C. Rosse.

$4^{\circ}$. Washington, 1883.

Botany. Вy ЈонN MUIR.

Birds. By E. W. NeLson.

Fishes. By T. H. Bear.

CORY (C. B.). A Naturalist in the Magdalen Islands.

$4^{\circ}$. Boston, 1878.

CORY (C. B.). Birds of the Bahama Islands.

4\%. Boston, 1880.

COSTA (A.). Ricerche Entomologiche sopra i Monti Partenii.

8. Napoli, 1858.

COSTA (A.). Degl' Insetti che attaccano l'albero ed il frutto dell' Olivo, del Ciliegio, del Pero, \&c. $\quad 4^{\circ}$. Napoli, 1857. 2nd edition. $4^{\circ}$. Napoli, 1877.

COSTA (A.). Annuario del Museo Zoologico di Napoli. 8. Napoli, 1862-67.

COSTA (E. M. da). Elements of Conchology, or an Introduction to the Knowledge of Shells. $\quad 8^{\circ}$. London, 1776.

COSTA (G.). Fauna Salentina.

8.. Lecce, 1874.

COSTA (0. G.). Fauna di Aspromonte e sue Adiacenze.

$4^{\circ}$. Napoli, 1828.

COSTA (0. G.). Catalogo de Testacei delle due Sicilie.

$4^{\circ}$. Napoli, 1829.

COSTA (0. G.). Cenni Zoologici ossia Descrizione sommaria delle specie nuove di Animali. 12 . Napoli, 1834. 
COSTA (O. G.). Esercitazioni Accademiche degli aspirante Naturalisti. 8. Napoli, 1839.

COSTA (O. G.). Monografia degl' insetti ospitanti sull' ulivo e nelle olive. 2nd edition. $8^{\circ}$. Napoli, 1840.

COSTA (O. G.). Fauna Siciliana. Vol. I. Animali Vertebrati [rectius Invertebrati]. $4^{\circ}$. Napoli, 1840.

COSTA (0. G.). Microdoride Mediterranea.

8. Napoli, 1861.

COSTA (0. G. \& A.). Fauna del Regno di Napoli. I.-XI. 4\%. Napoli, 1839-1881.

COSTE (M.). Nidification des Epinoches et des Epinochettes.

$4^{\circ}$. Paris, 1846.

COSTE (P.). See Strveys \& Exploratrons: France.

COUCH (J.). A Cornish Fauna ; being a Compendium of the Natural History of the County. $\quad 8^{\circ}$. Truro, 1838.

COUCH (J.). Illustrations of Instinct, deduced from the habits of British Animals.

8. London, 1847.

COUCH (J.). A History of the Fishes of the British Islands. 4 vols. $8^{\circ}$. London, 1862-65.

COCCH (J.). The History of Polperro, with additions by T. Q. Covch. $8^{\circ}$. Truro \& London, 1871.

COUES (E.). A Key to North-American Birds. roy. $8^{\circ}$. Salem, 1872.

COUES (E.). The Birds of the North-West.

8. Washington, 1874.

See Surteys \& Explorations: U.S., Washington.

COUES (E.). Abstract of Results of a study of the genera Geomys and Thomomys. $\quad 4^{\circ}$. Washington, 1875. 
COUES (E.). Furbearing Animals : A Monograph of the North-American Mustelidae. 8. Washington, 1877. See Surveys \& Explorations: U. S., Washington.

COUES (E.). New England Bird Life. From MS. of W. A. Stearns. 8. Boston, 1881. [In progress. $]$

COUES (E.) \& ALLEN (J. A.). Monographs of NorthAmerican Rodentia. $4^{\circ}$. Washington, 1877.

COUES (E.). Seo Surveys \& Explorations: U.S., WASHINGTON.

COWLEY (Capt.). See DaMprer (W.).

COX (H. E.). A Handbook of the Coleoptera, or Beetles, of Great Britain and Ireland. 2 vols. 8०. London, 1874.

CRAMER (P.). Papillons exotiques de l'Asie, de l'Afrique et de l'Amérique. 4 vols. $4^{\circ}$. Amst., Utrecht, 1779-83. Vol. 5. Supplement, par C. StoLL. 1787.

CRESPON (J.). Ornithologie du Gard et des pays circonvoisins. 8. Nismes, 1840.

CRESSON (E. T.). See Survers \& Explorations: U.S., WASHINGTON.

CREUTZER (C.). Entomologische Versuche.

8. Wien, 1799.

CROSSE (H.). See Survers \& Explorations : France.

CROSSE (H.) \& MARIE (E.). Catalogue des Cones de la Nouvelle-Calédonie et des Lles qui en dépendent.

$$
\text { 8. Paris, } 1874 .
$$

CROTCH (G. R.). Catalogue of British Coleoptera. 1st \& 2nd editions. $12^{\circ}$. Cambridge, $1863 \& 1871$.

CROTCH (G. R.). Synopsis Coleopterorum Europæ.

8. London, 1871. 
CROTCH (G. R.). Check List of the Coleoptera of America.

$8^{\circ}$. Salen, Mass., 1874.

CROTCH (G. R.). Revision of the Coccinellidae.

8. London, 1874.

CROUCH (E. A.). An illustrated Introduction to Lamarck's Conchology. $4^{\circ}$. London, 1826.

CUBIERS (S. L. P.). Histoire Abrégée des Coquillages de Mer, par S... L.... P... C... l'aîné.

4․ Versailles, 1798.

CUMBERLAND (G.). Remarkable fossil Encrinites and their connecting links.

8. Bristol, 1826.

CUNI Y MARTORELL (M.) \& MARTORELL Y PEÑA (MI.). Catálogo metódico y razonado de los Coleópteros observandos en Catalaña.

$8^{\circ}$. Barcelona, 1876.

CUNÍ Y MARTORELL (M.). Catálogo metódico y razonado de los Lepidópteros .... de Barcelona, .... y de Catalaña.

$8^{\circ}$. Barcelona, 1874.

CUNNiNghajI (D. J.). See ' Challenger.'

CUNNINGHAM (R. O.). Notes on the Natural History of the Strait of Magellan and west coast of Patagonia.

8. Edinburgh, 1871.

'CURACCOA.' Cruise of H.M.S. 'Curaçoa' among the South Islands in 1865. By J. L. Brenchley. roy. $8^{\circ}$. London, 1873.

CURTIS (J.). British Entomology, 1823-40.

Reissue. 8 vols. $8^{\circ}$. London, 1862.

CURTIS (J.). Guide to an arrangement of British Insects. 8. . London, 1829. 2nd edition. $8^{\circ}$. London, 1873.

CURTIS (J.). Guide to an arrangement of British Insects. With MS. annotations by J. C. DALE.

8. . London, 1829. 
CURTIS (J.). The Genera of British Lepidoptera.

$4^{\circ}$. London, 1858.

CURTIS (J.). Farm Insects.

8. London, 1883.

[CUR'TIS (W.).] Instructions for collecting and preserving Insects.

8. London, 1771.

CURTIS (W.). Fundamenta Entomologiæ: or an Introduction to the Knowledge of Insects. $8^{\circ}$. London, 1772.

CUVIER (F.). Les Dents des Mammifères.

80. Strasbourg \& Paris, 1825.

CUVIER (F.). De l'Histoire naturelle des Cétacés.

8.. Paris, 1836.

CUVIER (F.). Histoire Naturelle des Mammifères.

See Geoffroy-St.-Hilaire (I.).

CUVIER (G.). Tableau élémentaire de l'Histoire Naturelle des Animaux.

8. Paris, 1798.

CUVIER (G.). Leçons d'Anatomie comparée.

8. Paris, 1800.

CUVIER (G.). Le Règne Animal.

4 vols. $8^{\circ} . \quad$ Paris, 1817.

CUVIER (G.). Le Règne Animal. Vol. III. Crustacés, Arachnides, Insectes, par P. A. Latreille.

8.. Paris, 1817.

CUVIER (G.). Recherches sur les Ossemens fossiles.

7 vols. $4^{\circ}$. Paris, 1821-24.

CUVIER (G.). The Animal Kingdom, by Edward GriffitH. 16 vols. $8^{\circ}$. London, 1827-33.

CUVIER (G.). Le Règne Animal.

5 vols. $8^{\circ}$. Paris, 1829.

CUVIER (G.). Le Règne Animal.

20 vols. $8^{\circ}$. Paris, 1836-46. 
CUVIER (G.). The Animal Kingdom of the Baron Covier. Edited by W. MacGillivray.

[Forms vol. ii. of the Edinburgh Journal of Natural History.]

CUVIER (G.). See Comte (A.).

CUVIER (G.). Brief an C. H. Pfaff. (Ed. W. F. G. BeHn.) $8^{\circ}$. Kiel, 1845.

CUVIER (G.). See Gú́rin-MÉnéville (F. E.).

CUVIER (G. L.). Mémoires pour servir à l'histoire et à l'anatomie des Mollusques.

4. Paris, 1817.

CUVIER (G.) \& VALENCIENNES (A.). Histoire Naturelle des Poissons. 22 vols. $8^{\circ}$. Paris, 1828-49. Another copy with coloured plates.

22 vols. text. 4 vols. plates. $8^{\circ}$.

Also 1 vol. obl. fol. Paris, 1828-49.

CZENPINSKI (P.). Totius Regni Animalis Genera.

8. Viennoe, 1778.

CZERMAK (J.). Zur Orientirung im Gesammtgebiete der Zoologie.

8.. Leipzig, 1855.

DABRY DE THIERSANT (P.). La Pisciculture et la Pêche en Chine. Introduction par J. L. Soubeiran. $4^{\circ}$. Paris, 1872.

DAHL (G.). Coleoptera und Lepidoptera : ein systematisches Verzeichniss.

8. Wien, 1823.

DAHLBOM (A. G.). Monographia Chrysidum Sueciæ.

8. Londini Gothorum, 1829.

DAHLBOM (A. G.). Monographia Pompilorum Sueciæ.

8. Londini Gothorum, 1829. 
DAHLBOM (A.G.). Exercitationes Hymenopterologicæ.

8. Londini, 1831-33.

DAHLBOM (A. G.). Clavis novi Hymenopterorum Systematis. $4^{\circ}$. Lundce, 1835.

DAHLBOM (A. G.). Kort Underrättelse om scandinaviska Insekters. 8.. Lund, 1837.

DAHLBOM (A. G.). Synopsis Hymenopterologiæ scandinavicæ.

40. Lund, 1839-40.

DAHLBOM (A. G.). Dispositio Methodica Specierum Scandinavicarum ad Familias Hymenopterorum Naturales pertinentium. Pars I. Sphex.

8. Lundae, 1842.

DAHLBOM (A. G.). Onychia.

8.. Lund, 1842.

DAHLBOM (A. G.). Dispositio Methodica Specierum Hymenopterorum.

8.. Lundae, 1845.

DAHLBOM (A. G.). Hymenoptera Europæa.

2 vols. $8^{\circ} . \quad$ Lund, 1843-45, and Berolini, 1854.

DAHLBOM (A.G.). Zoologiska Studier. 8º Lund, 1856.

DALLAS (W. S.). List of Hemipterous Insects in the British Museum. $12^{\circ}$. London, 1851-52.

See Museum (British).

DALIAS (W. S.). Elements of Entomology.

8. London, 1857.

DALlas (W. S.). See Siebold (C. T. E. von).

DALMAN (J. W.). Analecta Entomologica.

$4^{\circ}$. Holmice, 1823.

DALMAN (J. W.). Ephemerides Entomologicæ.

8. Holmice, 1824.

DALYELL (J. G.). Rare and remarkable Animals of Scotland. 2 vols. $4^{\circ}$. London, 1847.

DALYELL (J. G.). The Powers of the Creator displayed in the Creation. 3 vols. $4^{\circ}$. London, 1851-58. 
DAMPIER (W.). A collection of Voyages. [DAMPIER, Wafer, Cowley, Sharp, Wood, and Roberts.]

4 vols. $8^{\circ}$. L.ondon, 1729.

DANA (J. D.). On Coral Reefs and Tslands.

8. ${ }^{\circ}$ New York, 1853.

DANA (J. D.). Corals and Coral Islands.

8. London, 1875.

DANA (J. D.). Zoophytes.

Text, $4^{\circ}$. Plates, fol. Philadelphia, 1848-49.

See Survers \& Explorations: U.S., Philadelphia.

DanCUS (le Roi). Le Livre du Roi Dances suivi d'un traité de Fauconnerie. [Reprint.] 12 . Paris, 1883.

DANDOLO (Count). The Art of Rearing Silk-Worms. Translated from the work of Count DandoLo.

8. London, 1825.

DANIELL (S.). African Scenery and Animals.

fol. London, 1804.

DANIELL (S.). A Picturesque Illustration of the scenery, animals, and native inhabitants of the Island of Ceylon.

fol. London, 1808.

DANIELL (W.). Sketches representing the Native Tribes, Animals, and Scenery of Southern Africa.

$4^{\circ}$. London, 1820.

DANIELL (W.). Elephant-hunting in the Island of Ceylon.

$8^{\circ}$. London, 1835.

DANIELSSEN (D. C.). See SARs (M.).

DANIELSSEN (D. C.). See Surveys \& Explorations: Norway.

DANSKY (J.) \& KOSTENITSCH (J.). Ueber die Entwickelungsgeschichte der Keimblätter und des Wolff'schen Ganges im Hühnerei. $\quad 4^{\circ}$. St. Pétersbourg, 1880.

DARRU (A.). See Agnély (Dr.). 
DARWIN (C.). A Monograph of the Cirripedia.

See Ray SocIETy.

2 vols. $8^{\circ}$. London, 1851-54.

DARWIN (C.). The Origin of Species by means of Natural Selection. $8^{\circ}$. London, 1866.

DARWIN (C.). The Descent of Man. $8^{\circ}$. London, 1871. 2nd edition. $8^{\circ}$. London, 1883.

DARWIN (C.). Expression of the Emotions in Man and Animals. 8. London, 1873.

DARWIN (C.). The Structure and Distribution of Coral Reefs. 2nd edition. $8^{\circ}$. London, 1874.

DARWIN (C.). The Formation of Vegetable Mould through the action of Earthworms. $\quad 8^{\circ}$. London, 1881.

DARWIN (C.). See 'Beagle.'

DARWIN (C.). See Weismann (A.).

DAUBENTON (L. J. M.). Les Animaux Quadrupèdes et Cétacés. $4^{\circ}$. Paris, 1782.

DAUBENTON (L. J. M.). Les Animaux Quadrupèdes Oripares et les Serpens. 4\%. Paris, 1784.

D’AUBUSSON (L. M.). Les Oiseaux de la France. Part I. $4^{\circ}$. Paris, 1883. [In progress.]

DAUDIN (F. M.). Recueil de Mémoires et de Notes sur des espèces inédites ou peu connues de Mollusques, de Vers et de Zoophytes.

$12^{\circ}$. Paris, 1800.

DAUDIN (F. M.). Histoire Naturelle génerale et particulière des Reptiles. $\quad 8$ vols. $8^{\circ}$. Paris, 1802-03. [Forming part of Euvres de Buffon by C. S. Sonnini.]

DAUMAS (E.). The Horses of the Sahara, and the Manners of the Desert. With commentaries by the Emir ABD-EL-KadER. Translated from the French by J. HutTon. 8. London, 1863. 
DAVAINE (C.). Recherches sur l'Anguillule du blé Niellé.

$8^{\circ}$. Paris, 1857.

DAVAINE (C.). Traité des Entozoaires.

8. $\quad$ Paris, 1877.

DAVENPORT (J.) \& COMELATI (G.). See Dictionaries.

DAVID (A.). Journal de mon troisième voyage d'exploration dans l'Empire Chinois. 2 vols. $8^{\circ}$. Paris, 1875.

DAVID (A.) \& OUSTALET (E.). Les Oiseaux de la Chine. 2 vols. $8^{\circ}$. Paris, 1877.

DAVidson (T.). See 'Challenger.'

DAVIES (J. B.). The Practical Naturalist's Guide.

$12^{\circ}$. Edinburgh, 1858.

DAVIS (J. B.). Thesaurus Craniorum. Catalogue of the Skulls of the various Races of Man. With Supplement.

2 vols. $8^{\circ}$. London, 1867-75.

DAVIS (J. B.) \& TURNHAM (J.). Crania Britannica. (Decade I.-VI.)

$4^{\circ}$. London, 1856-65.

DAVY (H.). Salmonia ; or Days of Fly-fishing.

4th edition. $12^{\circ}$. London, 1851.

DAVY (J.). The Angler in the Lake District.

8. London, 1857.

DAVY (J.). Physiological Researches.

8.. London, 1863.

DAWSON (J. F.). Carnivorous Ground-Beetles indigenous to the British Isles.

8. London, 1854.

DAY (F.). The Fishes of Malabar. $\quad 4^{\circ}$. London, 1865.

DAY (F.). Report on the Freshwater Fish and Fisheries of India and Burma. roy. $8^{\circ}$. Calcutta, 1873. 
DAY (F.). The Sea-Fishes of India and Burma.

$8^{\circ}$. Calcutta, 1873.

DAY (F.). The Fishes of India.

2 vols. $4^{\circ}$. London, 1875-78.

DAY (F.). The Fishes of Great Britain and Ireland.

Parts I.-VII. roy. $8^{\circ}$. London, 1880-83. [In progress.]

DAY (F.). See Fisheries: London. International Fisheries Exhibition, 1883.

DAY (F.). See SuRvers \& Explorations : Yarkand.

DEBEY (M.). Beiträge zur Lebens- und Entwickelungsgeschichte der Rüsselkäfer aus der Familie der Attelabiden. $4^{\circ}$. Bonn, 1846.

DEEN (J. A. Van). Anatomische Beschreibung eines monströsen, sechsfüssigen Wasser-Frosches (Rana esculenta).

$4^{\circ}$. Leiden, 1838.

DEGLAND (C. D.). Ornithologie Européenne.

2 vols. $8^{\circ}$. Paris et Lille, 1849.

DEGLAND (C. D.) \& GERBE (Z.). Ornithologie Européenne. Deuxième édition. 2 vols. $8^{\circ}$. Paris, 1867.

DEHNE (J. F. A.). Micromys agilis (Kleinmaus), ein neues Säugethier der Fauna von Dresden, aus der Ordnung der Nager.

$12^{\circ}$. Dresden, 1841.

DEJEAN (Le Comte). Spécies Général des Coléoptères. (Vol. 6 by C. Aube.) 6 vols. $8^{\circ}$. Paris, 1825-38.

DEJEAN (Le Comte) \& BOISDUVAL (J. A.). Iconographie et Historre naturelle des Coléoptères d'Europe.

5 vols. $8^{\circ}$. Paris, 1832-37.

DEJEAN (Le Comte). Catalogue des Coléoptères.

$1^{\text {re }}$ et $3^{\text {me }}$ éditions. $8^{\circ}$. Paris, 1821-37. 
DE LAPORTE (F. L. Comte de Castelnau) \& BRULLE (A.). Histoire naturelle des Insectes Coléoptères.

4 vols. $8^{\circ}$. Paris, 1840.

DE Laporte (F. L.). See Castelnat (F. L. Comte de).

DELESSERT (A.). Souvenirs d'un Voyage dans l'Inde exécuté de 1834 à 1839 .

8.. Paris, 1843.

DELESSERT (B.). Recueil de Coquilles décrites par Lamarck. fol. Paris, 1841.

DELEUZE (M.). Histoire et Description du Muséum Royal d'Histoire Naturelle. 2 vols. $8^{\circ}$. Paris, 1823.

DELPECH (A.). Les Trichines et la Trichinose chez l'homme et chez les animaux. 8. Paris, 1866.

DENNY (H.). Monographia Pselaphidarum et Scjdmænidarum Britanniæ.

8. Norwich, 1825.

DENNY (H.). Monographia Anoplurorum Britanniæ.

8. London, 1842.

DENNY (H.). List of the Anoplura or Parasitic Insects in the Collection of the British MIuseum.

$12^{\circ}$. London, 1852.

DENYS DE MONTFORT (P.). Conchyliologie systématique. 2 vols. $8^{\circ}$. Paris, 1808-10.

DERBY (Earl of). Catalogue of the Menagerie and Aviary at Knowsley. $\quad 4^{\circ}$. Liverpool, 1851.

DERBY (0. A.). See Musedm: Harvard College.

DERHEIMS (J. L.). Histoire naturelle médicale des Sangsues. $\quad 8^{\circ}$. Paris, 1825.

DESALLIER D'ARGENVILLE (A. J.). See Dezallier D'ARgENVILLE. 
DESBROCHERS DES LOGES (M.). Insectes Coléoptères du Nord de l'Afrique. Tenebrionides. $\quad 8^{\circ}$. Bone, 1881.

DESFONTAIN (R. L.). See Periodicals: London. Willughby Society.

DESHAYES (G. P.). Description des Coquilles fossiles des environs de Paris. 2 vols. $4^{\circ}$. Paris, 1824.

DESHAYES (G. P.). Traité élémentaire de Conchyliologies. Tom. I. Pts. I \&. II. Tom. II. Pt. I. \& Plates.

8. Paris, 1839-53.

[DESHAYES(G. P.).] Catalogus Concharum bivalvium in Museo Britannico.

12. London, 1854.

DESHAYES (G. P.). See Belanger (C.).

DESHAYES (G. P.). See Surveys \& Explorations : France.

DESMAREST (A. G.). Histoire naturelle des Tangaras, des Manakins et des Todiers.

fol. Paris, 1805.

DESMAREST (A. G.). Mammalogie : ou description des espèces de Mammifères. 2 vols. $4^{\circ}$. Paris, 1820-22.

DESMAREST (A. G.). Mémoire sur un noureau genre de Mammifères de l'ordre des Rongeurs, nommé Capromys.

4. Paris, 1822.

DESMAREST (A. G.). Considérations générales sur la classe des Crustacés.

$8^{\circ}$. Paris, 1825.

DESMAREST (A. G.). See Bosc (L. A. G.).

DESMaREST (A. G.). See Faune Française.

DESMOULINS (A.) \& MAGENDIE (F.). Anatomie des Systèmes Nerreux des Animaux à Vertèbres.

2 vols. and Atlas. $8^{\circ} \& 4^{\circ}$. Paris, 1825.

DESMOULINS (A.). Histoire Naturelle des Races Humaines.

8. Paris, 1826. 
DESMOULINS (C.). Essai sur les Sphérulites.

8. . Rordeaux, 1827.

DESMOULINS (C.). Ētudes sur les Ēchinides.

$8^{\circ}$. Bordeaux, 1835-37.

DESMOULINS (C.). Sur les Epines des Echinocidarites.

$8^{\circ}$. Bordeaux, 1869.

DES MURS (0.). Iconographie Ornithologique.

fol. Paris, 1849.

DES MURS (0.). Traité général d’Oologie.

8. Paris, 1860.

DES MURS (0.). See 'VENos.'

DESOR (E.). Die Pfahlbauten des Neuenburger Sees.

8. Frankfurt, 1866.

DESVIGNES (T.). British Ichneumonidoe in the British Museum.

12. London, 1856.

DEWHURST (H. W.). The Natural History of the order Cetacea.

8․ London, 1834.

DEWITZ (H.). Afrikanische Nachtschmetterlinge.

4․ - Halle, 1881.

DEYROLLE (H.). Description des Buprestides de la Malaisie recueillis par M. Wallace.

8.. Bruxelles \& Paris, 1864.

DEZALLIER D'ARGENVILLE (A. J.). L'Histoire Naturelle éclaircie. La Conchyliologie.

2nd edition. 4\%. Paris, 1757.

DEZALLIER D'ARGENVILLE (A. J.). La Conchyliologie, ou Histoire Naturelle des Coquilles.

2 vols. and Atlas. $4^{\circ}$. Paris, 1780.

DICKSON (0.). See FisherIes: London. International Fisheries Exhibition, 1883. 


\section{DICTIONARIES.}

English Language. By C. Richardson.

8. London, 1855.

English-Latin. By J. Roвson. sm. 4º. London, 1872.

Latin-English. By W. Surrer. $\quad 8^{\circ}$. London, 1855.

English-Greek, By C. D. Yonge. $4^{\circ}$. London, 1849.

Greek-English. By Liddeir and Scotr.

$4^{\circ}$. London, 1855.

English-French and French-English. By Fueming and Tibins. $\quad 2$ vols. $4^{\circ}$. Paris, 1875-77.

French-English and English-French. By L. ConTANSEAJ.

8. London, 1879.

English-German and German-English. By FLüGrL and Meissner. 2 vols. $8^{\circ}$. Leipzig, 1847.

English-Dutch and Dutch-English. By J. M. CALISCH. $8^{\circ}$. Tiel, 1875.

Italian-English and English-Italian. By Davenpor $\mathrm{r}$ and Comelati. $\quad 2$ vols. $8^{\circ}$. London, 1854.

Spanish-English and English-Spanish. By NeUxad and Baretti. Ed. Seoante.

2 vols. $8^{\circ}$. London, 1854.

Portuguese-English and English-Portuguese, abridged from VieYra.

2 vols. $12^{\circ}$. Paris, 1878.

English and Swedish. Ed. Tauchnitz.

12. Leipzig, n. d.

Engelsk-Svensk Ordbok af Nilsson, WidMark, och CoLtin.

Svensk-Engelsk Hand-Ordbok af Öran.

2 vols. $8^{\circ}$. Stockholm, 1875-81.

Dansk-norsk-engelsk Ordbog ved A. LARsEN.

8. Kjöbenhavn, 1880.

Russian, French, German, and English Languages. By C. P. Reiff.

2 vols. $8^{\circ}$. Carlsruhe, Leipzig, St. Petersburg, Paris, 1876-80.

Dictionnaire Classique d'Histoire Naturelle.

8․ Paris, 1823. 
DIESING (C. M.). Systema Helminthum.

2 vols. $8^{\circ}$. Vindobunce, 1850-51.

DIETRICH (K.). Beitrag zur Kenntniss der InsektenFauna des Kantons Zürich. (Käfer.)

$4^{\circ}$. Oberstrass, 1864.

DIGGLES (S.). Companion to Gould's Handbook; or Synopsis of the Birds of Australia.

2 vols. $4^{\circ} . \quad$ Brisbane, 1877.

DILLTYN (L. L.). See Motley (J.).

DILLWYN (L. W.). Descriptive Catalogue of recent Shells. 2 rols. 80. London, 1817.

DILLWYN (L. W.). Fauna and Flora of Swansea.

$8^{\circ}$. Swansea, 1848.

DIMMOCK (G.). The Anatumy of the Mouth-parts \&c. of Diptera.

$4^{\circ}$. Boston, 1881.

DISCONZI (F. de). Entomologia Vincentina.

8. Padova, 1865.

DISTANT (W. L.). Rhopalocera malayana : a description of the Butterflies of the Malay Peninsula.

Parts I.-V. $\quad 4^{\circ}$. London, 1882-83.

[In progress.]

Distant (W. L.). See Surteys axd Explorations: YARKAND.

DOBSON (G. E.). Monograph of the Asiatic Chiroptera. $8^{\circ}$. London, 1876.

DOBSON (G. E.). Catalogue of the Chiroptera in the Collection of the British Museum. $\quad 8^{\circ}$. Lonclon, 1878.

DOBSON (G. E.). A Monograph of the Insectivora.

Parts I. \& II. $4^{\circ}$. London, 1882-83.

[In progress.] 
DOBSON (G. E.). See Surveys \& Explorations: Yarkand.

DODERLEIN (P.). Prodromo della Fauna Ittiologica della Sicilia.

4.. Palermo, 1878-79.

DODERLEIN (P.). Prospetto metodico ... . di Pesci.... della Sicilia ; .... e Catalogo delle .... preparazioni tassidermiche ed anatomiche nel Museo .... di Palermo.

4. Palermo, 1878-79.

DODERLEIN (P.). Manuale Ittiologico del Mediterraneo. $4^{\circ}$. Palermo, 1879-81.

DOGIEL (J.). Anatomie und Physiologie des Herzens der Larve von Corethra plumicornis.

$4^{\circ}$. St. Petersburg, 1877.

DOHRN (A.). Der Ursprung der Wirbelthiere und das Princip des Functionwechsels. Genealogische Skizzen.

8.. Leipzig, 1875.

DOHRN (A.). See Fadna UNd Flora des Golfes von Neapel.

DOHRN (H.). See Fisherres : BerLin. Internationalo Fischerei-Ausstellung, 1880.

DOMBROWSKI (R. von). Der Fuchs. Monographischer Beitrag zur Jagd-Zoologie. $\quad$ 8. Wien, 1883.

DONATI (V.). Della Storia naturale marina dell' Adriatico.

4․ Venezia, 1750.

DONATI (V.). Essai sur l'Histoire Naturelle de la Mer Adriatique.

$4^{\circ}$. La Haye, 1758.

DONNDORFF (J. A.). Zoologische Beyträge. Die Säugethiere. 8. Leipzig, 1792.

DONOVAN (E.). Natural History of British Insects. 16 vols. $8^{\circ}$. London, 1792-1813.

DONOVAN (E.). Natural History of British Birds. 10 vols. $8^{\circ}$. London, 1794-1818. 
DONOVAN (E.). Natural History of British Shells. 5 rols. $8^{\circ}$. London, 1802-04.

DONOVAN (E.). Natural History of British Fishes.

5 vols. $8^{\circ}$. London, 1802-06.

DONOVAN (E.). The Naturalist's Repository.

5 vols. $8^{\circ}$. London, 1834.

DONOVAN (E.). Natural History of the Insects of China. Ed. J. O. WESTWOOD. 4. London, $18+2$.

DONOVAN (E.). Natural History of the Insects of India. Ed. J. 0. WestW0od. $4^{\circ}$. London, 1842.

Doran (A.). See Museum : Royal College of Surgeons.

D’ORBIGNY (A.). See Orbigny (A. d').

DORFMEISTER (G.). Ueber den Einfluss der Temperatur bei der Erzeugung der Schmetterlings-Varietäten.

8․ Graz, 1880.

DORIA (G.). See Prriodicals: Genoa and Modena, Archivio per la Zoologia.

DÖRING (R. von). Die Schlesischen Tagfalter.

$4^{\circ}$. Brieg, 1851.

DÖRING (R. von). See Periodicals : Brieg.

DOUBLEDAY (H.). A Synonymic List of British Lepidoptera. 1st and 2nd editions.

2 rols in $1.8^{\circ}$. London, 1850-59.

DOUBLEDAY (E.) \& WESTWOOD (J. 0.). The Genera of Diurnal Lepidoptera.

2 vols. fol. London, 1846-50.

DOUGLAS (J. W.). The World of Insects.

$8^{\circ}$. London, 1856 。

DOUGLAS (J. W.) \& SCOTT (J.). The British Hemiptera.

See Ray Socierr.

8. . London, 1865. 
DOUGLAS (J. W.) \& SCOTT (J.). A Catalugue of British Hemiptera. 8. . London, 1876.

DRAPARNAUD (J. P. R.). Histoire naturelle des Mollusques terrestres et fluviatiles de la France. 4\% . Paris, 1805.

DRESSER (H. E.). See Sharpe (R. B.).

DROSTE HÜLSHOFF (Baron F. von). Die Vogelwelt der Nordseeinsel Borkum. 8․ Münster, 1869.

DROUET (H.). Essai sur les Mollusques Terrestres et Fluviatiles de la Guyane Française. $8^{\circ}$. Paris, 1859.

DROUET (H.). Eléments de la Faune Açoréenne. $4^{\circ}$. Paris, 1861.

DRUMMOND (W. H.). The Large Game, and Natural History of South and South-East Africa.

$8^{\circ}$. Edinburgh, 1875.

DRURY (D.). Illustrations of Natural History.

3 vols. $4^{\circ}$. London, 1770-82.

DRURY (D.) \& WESTWOOD (J. O.). Illustrations of Exotic Entomology. Ed. J. O. Westwood.

3 vols. $4^{\circ}$. London, 1837.

DRYANDER (J.). Catalogus Bibliothecæ historico-naturalis Josephi Banks. 5 vols. $8^{\circ}$. London, 1798-1800.

DUBAR (J. V. P.). Ostéographie de la Baleine.

8. Bruxelles, 1828.

DUBOIS (A.). Conspectus systematicus et geographicus Avium Europæarum.

8. . Bruxellis, 1871.

DUBOIS (A.). Lépidoptères de l'Europe.

[In progress.]

roy. $8^{\circ}$. Bruxelles, 1881-83.

DUBOIS (C. F.), Planches Coloriées des Oiseaux de la Belgique et de leurs œufs.

3 vols. $8^{\circ}$. Bruxelles, 1854-60. 
DUBOIS (C. F.). Les Oiseaux de l'Europe et leurs œufs. 2 vols. $8^{\circ}$. Bruxelles, 1868-72.

DUBOIS (C. F. \& A.). Les Lépidoptères de la Belgique. roy. 8vo. Bruxelles, Leipzig, \& Paris, 1859-60. Continued as 'Les Lépidoptères de l'Europe.' roy. $8^{\circ}$. Bruxelles, 1877-83.

DU BOIS-REYMOND (E.). See Periodicals: Berlin and LeIPZig.

DUBRaviUS (J.). De Piscinis et Piscium qui in eis aluntur naturis libri quinque.

$12^{\circ}$. 1559 .

DUBRAVIUS (J.). De Piscinis. Lib. V. Cum præfationibus cura H. ConringIr. sm. $4^{\circ}$. Helmestadii, 1671.

DU BUS DE GISIGNIES (le Vte. B.). Catalogue des Livres.

8. Bruxelles, 1876.

DUCHAMP (G.). Recherches sur les Ligules.

8. Paris, 1876.

DUCHASSAING (P.). Animaux Radiaires des Antilles.

8. Paris, 1850.

DUCHASSAING (P.) \& MICHELOTTI (J.). Mémoire sur les Coralliaires des Antilles. $\quad 4^{\circ}$. Turin, 1860.

Supplement, $4^{\circ}$. Turin, 1864.

DUCLOS (P. L.). Histoire Naturelle de Coquilles Univalves Marines. Genre Olive.

fol. Paris, 1835 .

DUFF (R. W.). See Fishertes : London.

DUFTSCHMID (K.). Fauna Austriæ.

3 vols. $8^{\circ}$. Linz \& Leipzig, 1805-25.

DUGÈS (A.). Traité de Physiologie comparée de l'Homme et des Animaux. $\quad 3$ vols. $\quad 8^{\circ}$. Montpellier, 1838-39.

DUHAMEL DU MONCEAU (H. L.) \& DE LAMARRE (L. H.). Traité général des Pêches.

3 vols. fol. Puris, 1769-82. 
DUJARDIN (F.). Histoire Naturelle des Zoophytes. Infusoires. Texte et Atlas. $8^{\circ}$. Paris, 1841.

DUJARDIN (F.) \& HUPÉ (H.). Histoire naturelle des Zoophytes. Echinodermes.

8. Paris, 1862.

DUJARDIN (F.). Histoire naturelle des Helminthes ou Vers intestinaux.

8. Paris, 1845 .

DUMÉRIL (A.). Histoire naturelle des Poissons.

8. Paris, 1865.

DUMÉril (A.). See Survers \& Explorations: France.

DUMERIL (A.). See 'VENUs.'

DUMÉRIL (A. M. C.). Zoologie Analytique.

8.. Puris, 1806.

DUMÉRIL (A.M. C.). Yémoires de Zoologie et d'Anatomie Comparée.

8. Paris, 1807.

DUMÉRIL (A. M. C.). Dissertation sur les Poissons qui se rapprochent le plus des animaux sans vertèbres.

4\%. Paris, 1812.

DUMÉRIL (A. M. C.). Considérations Générales sur la Classe des Insectes. $8^{\circ}$. Paris, 1823.

DUMÉRIL (A. M. C.). Ichthyologie analytique.

4\%. Paris, 1856.

DUMÉRIL (A. M. C.) \& BIBRON (G.). Erpétologio générale.

9 vols. Paris, 1834-37.

DUMÉRIL (C. \& A.). Catalogue méthodique de la collection des Reptiles.

8.. Paris, 1851.

DUNCAN (P. M.). Cassell's Natural History. Edited by P. M. Duncan. $\quad 6$ vols. $4^{\circ}$. London, 1883.

DUNCAN (P. M.). See SuRters \& Explorations : YARKAND. 
DUNCAN (P. M.) \& SLADEN (W. P.). A Memoir on the Echinodermata of the Arctic Sea to the West of Greenland. $4^{\circ}$. London, 1881.

DUNKER (W.). See Novitates Conchologicz.

DUNN (R.). The Ornithologist's Guide to Orkney and Shetland.

8. London,-1837.

DU PETIT-THOUARS (A.). See 'Veñs.'

DUPONCHEL (P. A. J.). Catalogue Méthodique des Lépidoptères d'Europe. $8^{\circ}$. Paris, 1844.

DUPONCHEL (P.A.J.) \& GUENEE (A.). Iconographio et Histoire Naturelle des Chenilles.

2 vols. $8^{\circ}$. Paris, 1849.

DUPONCHEL (P. A. J.). See Godart (I. B.).

DUPONT (É.). Notice sur la vie et les travaux de PierreHenri Nyst. $12^{\circ}$. Bruxelles, 1882.

DUPUY (D.). Histoire Naturelle des Mollusques. Planches par J. Delarde. $\quad 4^{\circ}$. Paris, 1847-52.

DURAZZO (C.). Degli Uccelli Liguri.

8. Genova, 1810.

DURSY (E.). Zur Entwicklungsgeschichte des Kopfes des Menschen und der höheren Wirbelthiere.

Text and Atlas. 8 $^{\circ}$. Tübingen, 1869.

DUSEIGNEUR-KLEBER (MI.). Le Cocon de Soie.

8. Paris, 1875.

DUTTENHOFER (F. M.). Seo RÁpp (W. L.) \& DuttenHOFER (F. M.).

DUVERNOY (G. L.). De l'organisation des Echinodermes.

$4^{\circ}$. Paris, 1848.

DWIGH'T (T.). Description of Balcenoptera musculus.

$4^{\circ}$. Boston, 1872. 
D’YAUVILLE (M.). Traité de Venerie.

4. Paris, 1788.

DYBOWSKI (B. N.). Versuch einer Monographie der Cyprinoiden Livlands. $\quad 8^{\circ}$. Dorpat, 1862.

DYBOWSKI (B. N.). Beiträge zur näheren Kenntniss der Gammariden. $4^{\circ}$. St. Petersburg, 1874.

DYBOWSKI (W.). Dio Gastropoden-Fauna des BaikalSees. $4^{\circ}$. St. Petersburg, 1875.

DYBOWSKI (W.). Die Spongien des Russischen Reiches。 $4^{\circ}$. St. Petersburg, 1880.

DYBOWSKI (W.). Studien über die SüsswasserSchwämme des Russischen Reiches.

$4^{\circ}$. St. Petersburg, 1882.

$\mathbb{E}$.

EBERT (F. A.). Bibliographical Dictionary.

4 vols. $8^{\circ}$. Oxford, 1837.

EBERT (R.). Zur Systematisierung des Thierreichs.

8. Dresden, 1868.

EBERTH (C. J.). Untersuchungen über Nematoden.

$4^{\circ}$. Leipzig, 1863.

EBERTH (C. J.). Zur Kenntniss der bacteritischen Mykosen.

$4^{\circ}$. Leipzig, 1872 .

EBRARD (E.). Nouvello Monographie des Sangsues Médicinales.

8.. Paris, 1857.

ECKER (A.). Icones Physiologicæ. Erläuterungstafeln zur Physiologie.

4. Leipzig, 1851-59.

ECKER (A.). Die Anatomie des Frosches.

8o. Braunschweig, 1864-82. 
ECKER (A.). Crania Germaniæ meridionalis occidentalis. $4^{\circ}$. Freiburg, 1865.

ECKHARD (C.). Beiträge zur Anatomie und Physiologie. 10 vols. in 5. $4^{\circ}$. Giessen, 1858-83.

ECKHARD (C.). See Hermand (L.). Handbuch der Physiologie.

ECKHEL (G. $\nabla$.$) . Der Badeschwamm in Rücksicht auf$ die Art seiner Gewinnung, die geographische Verbreitung und locale Variation. $\quad 8^{\circ}$. Trieste, 1873.

EDINBURGH (H.R.H. the Duke of). See Fisherres: London. International Fisheries Exhibition, 1883.

EDWARDS (G.). A Natural History of uncommon Birds, and of some rare and undescribed Animals.

4 vols. $4^{\circ}$. London, 1743.

EDWARDS (G.) \& CATESBY (M.). See Seligmann (J.M.).

EDWARds (H.). See Periodicals: New York. Papilio.

EDWARDS (W. H.). The Butterflies of North America. $4^{\circ}$. Philadelphia, 1868-72. 2nd Series. $4^{\circ}$. New York, 1874-80. [In progress.]

EDWARDS (W. H.). See Surveys \& Exploratrons: U. S., Washington.

EGERTON (P. G.). A Systematic and Stratigraphical Catalogue of the Fossil Fish in the Cabinets of Lord Cole and Sir Philip Grey Egerton. $\quad 4^{\circ}$. London, 1837.

EHLERS (E.). Die Borstenwürmer (Annelida Chætopoda).

$4^{\circ}$. Leipzig, 1864-68.

EHLERS (E.). Die Esper'schen Spongien in der zoologischen Sammlung der k. Universität Erlangen.

$4^{\circ}$. Erlangen, 1870.

EHLERS (E.). See Keferstern (W.). 
EHRENBERG (C. G.). Weitere Entwicklung des Hyalonema \&c.

$8^{\circ}$. Berlin, 1867.

EHRENBERG (C. G.). Die Corallenthiere des Rothen Meeres. $4^{\circ}$. Berlin, 1834 .

EHRENBERG (C. G.). Die Infusionsthierchen als Vollkommene Organismen.

fol. Leipzig, 1838.

EHRENBERG (C. G.) \& HEMPRICH (F. G.). Symbolæ Physicæ. fol. Berolini, 1828.

EICHHOFF (W.). Die Europäischen Borkenkäfer.

8. Berlin, 1881.

EICHWALD (E.). De Selachis Aristotelis, zoologiæ geographicæ specimen.

$8^{\circ}$. Vilnae, 1819.

EICHWALD (E.). Zoologia specialis quam expositis animalibus tum vivis, tum fossilibus potissimum Rossiae in universum, et Poloniae in specie.

3 vols. in $2.8^{\circ}$. Vilnae, 1829 .

EICHWALD (E.). Catalogus Musæi Zootomici Imperatoriæ Academiæ Medico-Chirurgicæ Vilnensis.

$4^{\circ}$. Vilnae, 1835.

EICHWALD (E.). Fauna Caspio-Caucasia.

$4^{\circ}$. Petropoli, 1841.

EIMER (T.). Zoologischen Studien auf Capri.

Parts I. \& II. $4^{\circ}$. Leipzig, 1873-74.

EIMER (T.). Die Medusen, physiologisch und morphologisch auf ihr Nervensystem.

$4^{\circ}$. Tübingen, 1878.

EISELT (J. N.). Systematik und Literatur der Insectenkunde. 8. Leipzig, 1836.

EKHOFF (E.). Beskrifning öfver Benbyggnaden hos Glansfisken (Lampris guttatus). $\quad 8^{\circ}$. Stockholm, 1875.

EKSTRÖM (C. U.). Die Fische in den Scheeren von Mörkö.

8. Berlin, 1835. 
EKSTRÖMI (C. U.). See FrIes (B. F.).

ELDITT (H. L.). Die Metamorphose des Caryoborus (Bruchus) gonagra, Fabr. $\quad 4^{\circ}$. Königsberg, 1860.

ELLIOT (D. G.). A IIonograph of the Tetraoninae: fol. New York, 1865.

ELLIO'T (D. G.). A Lonograph of the Pittidae, or Family of Ant-Thrushes. fol. New York, 1867.

ELIIOT (D. G.). The Birds of North America.

fol. New York, 1869.

ELLIOT (D. G.). A Monograph of the Phasianidae.

2 vols. fol. NTew York, 1872.

ELIIOT (D. G.). A Monograph of the Paradiseidce.

fol. London, 1873.

ELLIOT (D. G.). Monograph of the Felidce.

fol. New York, 1878-83.

ELLIOT (D. G.). A Mronograph of the Bucerotidce, or Family of the Hornbills. fol. London, 1882.

ELLIOTT (H. W.). The Seal-Islands of Alaska. See Fisheries: Washington.

EILIS (J.). An Essay towards a Natural History of the Corallines. $4^{\circ}$. London, 1755.

ELLIS (J.). Essai sur l'histoire naturelle des Corallines. 4․ A la Haye, 1756.

ELLIS (J.). The Natural History of Zoophytes.

$4^{\circ}$. London, 1786.

ELLIS (W.). Polynesian Researches.

2nd edition. 4 vols. $16^{\circ}$. London, 1831-32. 
ELSAESSER (C. L.). See RAPP (W. L.) \& Elgaesser (C. L.).

EMERTON (J. H.). See HENz (N. M.).

EMERY (C.). Studii anatomici sulla Vipera Redii.

$4^{\circ}$. Milano, 1873.

EMERY (C.). See Fadna und Flora des Golfes von Neapel.

EMMONS (E.). Natural History of New York. (Agriculture, vol. $\nabla$.) Descriptions of the more common and injurious species of Insects. 4. Albany, 1854.

ENCYCLOPÉDIE MÉTHODIQUE. BoNNa TERRE (l'Abbé). Histoire naturelle des Poissons. Cétologio. Ophiologie. Ornithologie. Papilio. $4^{\circ} . \quad$ Paris, 1787. $4^{\circ}$. Paris, 1789. 4.. Paris, 1790. $4^{\circ}$. Paris, 1791. $4^{\circ}$. Paris, 1819.

ENGELMANN (W.). Bibliotheca Historico-Naturalis. 8. Leipzig, 1846.

ENGELMANN (W.). Bibliotheca Zoologica. See CARUs (J. V.) \& Engelmann (W.).

ENGELMANN (W.). See Hermand (L.). Handbuch der Physiologie.

ENGRAMELLE (D. J.). See Ernst (J. J.)。

ENYS (J. D.). See Butler (A. G.).

ERDL (M. P.). Die Entwickelung des Menschen und des Hühnchens im Eie. $4^{\circ}$. Leipzig, 1845-46.

ERDL (P.). Tafeln zur vergleichenden Anatomie des Schaedels. fol. München, 1841. 
'EREBUS' AND 'TERROR,' The Zoology of the Voyage of H.M.Ss., under the command of Captain Sir J. C. Ross, during the years 1839-43. $\quad 4^{\circ}$. London, 1844-75.

Mammalia. By J. E. Gray. 1844.

Birds. By G. R. Gray. 1844.

Appendix by R. B. Sharpe. 1875.

Reptiles. By J. E. Gray. 1845.

" Conclusion by A. GüNther. 1875.

Fishes. By Sir Jomn Richardson. 1844-48.

Crustacea. By E. J. Mrers. 1874.

Insects. By A. Whiте. 1844.

,$\quad$ By A. G. Butler. 1874.

Mollusca. By E. A. Surrtr. 1874.

ERHARD (Dr.). Fauna der Cykladen. 8․ Leipzig, 1858.

ERICHSON (G. F.). Genera et Species Staphylinorum Insectorum.

8. Berolini, 1840.

ERICHSON (W. F.). Die Käfer der Mark Brandenburg. 8. Berlin, 1837.

ERICHSON (W. F.). Entomographien.

$8^{\circ}$. Berlin, 1840.

ERICHSON (W. F.). Naturgeschichte der Insecten Deutschlands.

$8^{\circ}$. Berlin, 1848-68.

ERICHSON (W. F.). See Surteys \& Explorations: RUssia.

ERNST (J. J.). Papillons d'Europe, peints d'après nature par ERnst, . . . décrits par D. J. Engramelle.

8 vols. $4^{\circ}$. Paris, 1779-93.

ERXLEBEN (J. C. P.). Systema Regni Animalis.

8․ Lipsice, 1777.

ESCHRICHT (D.F.). De Organis quæ Respirationi et Nutritioni foetus Mammalium inserviunt.

$4^{\circ}$. Hafnice, 1837.

ESCHRICHT (D. F.). Anatomische Tntersuchungen über die Clione borealis.

$4^{\circ}$. Copenhagen, 1838. 
ESCHRICHT (D. F.). Chelyosoma macleayanum.

$4^{\circ}$. Kjöbenhavn, 1841.

ESCHRICHT (D. F.). Anatomisch-physiologische Untersuchung über die Bothryocephalen. $4^{\circ}$. Bonn, 1841.

ESCHRICHT (D. F.). Undersögelser over Hvaldyrene.

6 parts. $4^{\circ} . \quad$ Kö̈benhavn, 1844-48.

ESCHRICHT (D. F.). Ueber die nordischen Wallthiere. $4^{\text {. }}$ Leipzig, 1849.

ESCHRICHT (D. F.). See RAY Society.

ESCHRICHT (D. F.) \& REINHARDT (J.). Om Nordhralen (Baloena mysticetus). $\quad 4^{\circ}$. Kjöbenhavn, 1861.

ESCHSCHOLTZ (J. F.). Entomographien.

8.. Berlin, 1822.

ESCHSCHOLTZ (J. F.). System der Acalephen.

$4^{\circ}$. Berlin, 1829.

ESCHSCHOLTZ (J. F.). Zoologischer Atlas.

$4^{\circ}$. Berlin, 1829.

ESPER (E. J. C.). Die Schmetterlinge.

6 vols. $4^{\circ}$. Leipzig, 1829-39.

ESPER (E. J. C.). Die ausländischen Schmetterlinge.

$4^{\circ}$. Leipzig, 1830.

ESPER (J. F.). Ausführliche Nachricht von neuentdeckten Zoolithen unbekannter vierfüsiger Thiere, \&c.

$4^{\circ}$. Nürnberg, 1774.

ESPINAS (A.). Die thierischen Gesellschaften. Ed. W. Schloesser.

8. Braunschweig, 1879.

ETALLON (A.). Sur la Classification des Spongiaires du Haut-Jura, et leur distribution dans les étages.

8. Porrentruy, 1860.

EUDES-DESLONGCHAMPS (E.). Catalogue descriptif des Trochilidés.

8. Caen \& Paris, 1880. 
'EUGENIE.' Kongliga Srenska Fregatten 'Eugenies' Resa omkring Jorden under befäl af C. A. Virgin. Àren 1851-53.

Zoologi 1, Insecta. $4^{\circ}$. Stockholm, 1858-68. Annulata. C. H. Boheman. 1858.

Coleoptera. C. H. BoHeMran. 1859.

Hemiptera. C. STån. 1859.

Orthoptera. C. STÅr. 1860.

Lepidoptera. H. D. J. WALlengren. 1861.

Hymenoptera. A. E. Holmaren. 1868.

Diptera. C. G. Thомson, 1868.

EVANS (W. F.). British Libellulince; or, Dragon Flies.

8. London, 1845.

EVERSMANN (E.). Lacertæ imperii Rossici variis in itineribus meis observatæ. $4^{\circ}$. Moscow, 1834.

EVERSMANN (E.). Fauna Lepidopterologica VolgoUralensis. 8. Casani, 1844.

EWART (J.). The Poisonous Snakes of India.

$4^{\circ}$. London, 1878.

EXNER (S.). See Hermann (L.). Handbuch der Physiologie.

EYDOUX (F.) See 'FAVorite.'

EYDOUX \& SOULEYET, List of Mollusca collected by.

See MUseum (British).

$12^{\circ}$. London, 1855.

EYDOUX \& SOULEYET. See 'BonIte.'

EYFERTH (B.). Die Mikroskopischen Süsswasserbewohner in gedrängter Übersicht vorgeführt von B. E.

8. Braunschweig, 1877.

EYFERTH (B.). Die einfachsten Lebensformen.

$4^{\circ}$. Braunschweig, 1878.

EYTON (T. C.). A History of the Rarer British Birds.

$8^{\circ}$. London, 1836. 
EYTON (T. C.). Catalogue of Birds.

$12^{\circ}$. Wellington, Salop, 1856.

EYTON (T. C.). History of the Oyster and Oyster Fisheries.

8.. London, 1858.

EYTON (T. C.). Osteologia Avium.

4. Wellington, Salop, 1867.

EYTON (T. C.). Synopsis of the Anatida, or Duck tribe.

12․ Wellington, Salop, 1869.

EYTON (T. C.). Fishing Literature.

$12^{\circ}$. Wellington, Salop, 1871.

F.

FABER (C.). Der Bau der Iris des Menschen und der Wirbelthiere. 8. Leipzig, 1876.

FABER (F.). Prodromus der Isländischen Ornithologie oder Geschichte der Vögel Islands. $8^{\circ}$. Copenhagen, 1822.

FABER (F.). Ueber das Leben der hochnordischen Vögel. 8. Leipzig, 1825-26.

FABER (F.). Naturgeschichte der Fische Islands.

$4^{\circ}$. Frankfurt am M., 1829.

FABER (G. L.). The Fisheries of the Adriatic and the Fish thereof.

8.. London, 1883.

FABRE (J. H.). Faune Avignonaise. Insectes Coléoptères.

8. Avignon, 1870.

FABRE (J. H.). Souvenirs Entomologiques.

$12^{\circ}$. Paris, 1879.

FABRICIUS (J. C.). Systema Entomologiæ.

8. Flensburgi \& Lipsice, 1775.

FABRICIUS (J. C.). Genera Insectorum.

8․ Chilonii, 1776. 
FABRICIUS (J. C.). Philosophia entomologica.

8. Hamburgi, 1778.

FABRICICS (J. C.). Species Insectorum.

2 vols. $8^{\circ}$. Hamburgi, 1781.

FABRICIOS (J. C.). Mantissa Insectorum.

2 vols. $8^{\circ}$. Hafnice, 1787.

FABRICICS (J. C.). Entomologia systematica. 4 vols. and Index \& Supplement.

8. Hafnice, 1792-98.

FABRICIUS (J. C.). Systema Eleutheratorum.

2 vols. 80. Kitice, 1801.

FABRICICS (J. C.). Systema Rhynchotorum.

8․ Brunsvigce, 1803.

FABRICICS (J. C.). Systema Piezatorum.

80. Brunsviga, 1804.

FABRICIUS (J. C.). Systema Antliatorum.

$8^{\circ}$. Brunsvigoe, 1805 .

FABRICIUS (0.). Fauna Grœnlandica.

8. Hafrice \& Lipsice, 1780.

FÅHRaEUS (O. J.). See Bohemax (C. H.).

FAILLE (C. de la). Versuch über die Naturgeschichte des Maulwurfes. 12. Frankfurt \& Leipzig, 1778.

FAIRMAIRE (L.) \& LABOULBENE (A.). Faune Entomologique Française (Coléoptères). $\quad$ 120. Paris, 1854.

FALK (J. P.). Beyträge zur topographischen Kenntniss des Russischen Reichs. 3 vols. $4^{\circ}$. St. Petersburg, 1785.

FALLEN (C. F.). Observationes Entomologicæ.

$4^{\circ}$. Lundae, 1802-7.

FALLEN (C. F.). Monographia Cantharidum et Malachiorum Sueciæ.

$4^{\circ}$. Lunde, 1807. 
FALLEN (C. F.). Monographia Cimicum Sueciæ.

8.. Hafnice, 1818. Another edition. $8^{\circ}$. Hafnice, 1823.

FALLEN (C. F.). Hemiptera Sueciæ.

8. Londini Gothorum, 1829.

FALLÉN (C. F.). Monographia Tenthredinum Sueciæ. 8. Londini Gothorum, 1829.

FALLON (F.). Monographie des Oiseaux de la Belgique. 8. Namur \& Paris, 1875.

FARINES (M.) \& CARCASSONNE (M.). Mémoire sur un Cétacée. 8. Perpignan, 1829.

FATIO (V.). Les Campagnols du Bassin du Léman.

8. Bale et Genève, 1867.

FATIO (V.). Faune des Vertébrés de la Suisse.

Vols. I., III., IV. 8*. Genève \& Bale, 1869-72.

FAULKNER (H.). Elephant Haunts.

8. London, 1868.

FAUNE FRANCAISE ou histoire générale et particulière des Animaux qui se trouvent en France.

Texte, $8^{\circ}$. Planches, $4^{\circ}$. Paris, v. d.

Mammifères par A. G. Desmaregt.

Oiseaux par L. P. VieILLот.

Reptiles par A. G. Desmarest.

Poissons par H. M. De Blainville.

Insectes par A. Servielle, A. G. Desmarest, Lepeletier de Saint Fargead, A. G. De Blatnvilue et C. A. Walckenaer.

Vers, Mollusques et Zoophytes par H. M. De BraInVILLE.

FAUNA D' ITALIA. Edited by F. Vallardr.

Mammiferi per E. Cornalia.

roy. $8^{\circ}$. Milano, 1868-71.

Uccelli per T. SALVADoRI.

Pesci per G. Canestrini.

Rettili ed Amfibi per E. de Betra. 


\section{FAUNA JAPONICA.}

4 vols. fol. Lugduni Batavorum, 1838-50.

Mammifères par C. J. Tеммдаск. $18 \pm 7$.

Aves par C. J. Temminck et H. Schlegel. 1850.

Reptilia par C. J. Temminck et H. Schlegel. 1838.

Pisces par C. J. Temminck et H. Schlegel. 1850.

Crustacea par W. DE HAAN. 1850.

FAUNA UND FLORA DEs Golfes voN NeAPEL und der angrenzenden Meeres-Abschnitte, herausgegeben von der zoologischen Station zu Neapel. $\quad 4^{\circ}$. Leipzig, 1880-83.

I. Ctenophoræ von Dr. C. Сном. 1880.

II. Fierasfer von Dr. C. EMery. 1880.

III. Pantopoda von Dr. A. DoHRN. 1881.

IV. Corallina von Graf zu Solms-Laubach. 1881.

V. Chætognathen von Dr. B. Grassi. 1883.

VI. Caprelliden von Dr. P. Mayer. 1882.

VII. Cystoseiren von R. Valiante. 1883.

VIII. Bangiaceen von Dr. G. Berthold. 1882.

FAUVEL (A.). Lépidoptères du Calvados. $1^{\mathrm{e}}$ partie (Diurnes et Crépusculaires). $\quad 4^{\circ}$. Caen, 1863.

FAUVEL (A.). Notices Entomologiques.

Vol. 1 (pts. 1-5) and 2 pts. $(6,7)$. 80. Caen, 1862-78.

FAVANNE DE MONTCERVILLE. Dessins coloriés de Coquilles. 3 vols. $8^{\circ}$. Paris, n. d.

FAVANNE DE MONTCERVILLE. See DezalimR d'Argenville. La Conchyliologie.

'FAVORITE' (La). Voyage autour du Monde. Exécuté pendant les années 1830,1831,1832. Par M. Laplace. Atlas Hydrographique. fol. Paris, 1833. Atlas Historique. fol. Paris, 1835. Texte Historique. 4 vols. $8^{\circ}$. Paris, 1833.

Texte Zoologique. 1 vol. $8^{\circ}$. Paris, 1839. Mammifères. 1 Partie. Par F. Eydoux et Ladrent. 2 Partie. Par F. Eydodx et P. Gervais. Supplement. Lépidoptères Nouveaux. Par Baron Feisthamel.

FAXON (W.). Embryological Monographs. I. Crustacea. 4‥ Cambridge, Mass., 1882.

See Mureum: Hartard College. Memoirs. 
FAYRER (J.). The Thanatophidia of India; being a description of the Venomous Snakes of the Indian Peninsula. fol. London, 1874.

FAYRER (J.). The Royal Tiger of Bengal.

$8^{\circ}$. London, 1875.

FEHR (J. G.). Alphabetisches Verzeichniss aller in Boisduval's System der Europäischen Schmetterlinge.

8. Erlangen [1842].

FEISTHAMEL (Le Baron). See 'Favorite,

FELDER (C. \& R.). Lepidopterologische Fragmente.

$4^{\circ}$. Wien, 1859 .

FELDER (C. \& R.). See ' Novara.'

FENNELL (J. H.). A Natural History of British and Foreign Quadrupeds. $\quad 8^{\circ}$. London, 1841.

FENZL (J. G.). Conspectus Animalium Medicinalium.

8.. Patavii, 1834.

FERGUSON (D.). The Natural History of Redcar and its Neighbourhood. $12^{\circ}$. London, 1860.

FERMOND (C.). Mémoire sur la conservation et la reproduction des Sangsues.

$8^{\circ}$. Paris, 1851.

FERRARI (J. A. G.). Die Forst- und Baumzucht-schädlichen Borkenkäfer (Tomicides, Lac.) aus der Familie der Holzverderber (Scolytides, Lac.). $\quad 8^{\circ}$. Wien, 1867.

FERRARI (P. M.). See Periodiúals : Geneva and MoDeNa. Archivio per la Zoologica.

FÉRUSSAC (A. E. J. de). Tableaux systématiques des animaux mollusques.

fol. Paris, 1821.

FÉRUSSAC (A. E. J. de) \& ORBIGNY (A. d'). Histoire des Céphalopodes.

Texte \& Atlas. 2 vols. fol. Paris, 1835-48. 
FÉRUSSAC (J. B. L. de). Essai d'une méthode Conchyliologique.

FÉRUSSAC (J. B. L. de) \& DESHAYES (G. P.). Histoire naturelle générale et particulière des Mollusques.

See SAJder-Rajg (A.).

4 vols. fol. Paris, 1820-51.

fettig (l'Abbé). See Pexertmhoff (H. de).

FICHTEL (L. ..) \& MOLL (J. P. C. จ.). Testacea microscopica aliaque minuta ex generibus Argonauta et Nautilus.

[Second Title in German.] $\quad 4^{\circ}$. Wien, 1803.

FICK (A.). See Hermanx (L.). Handbuch der Physiologie.

FICKERT (C.). Myriopoden und Araneiden rom Kamme des Riesengebirges.

8. Breslau, 1875.

FIEBER (F. X.). Synopsis der Europäischen Orthopteren.

8․ Prag, 1854.

FIEBER (F. X.). Die Europäischen Hemiptera (Rhynchota Heteroptera).

8․ Wien, 1861.

FIEBER (F. X.). Katalog der Europäischen Cicadinen. 8․ Wien, 1872.

FIGUIER (L.). Les Insectes. 8. Paris, 1867.

FILIPPI (F. de) \& VERANY (G. B.). Sopra alcuni Pesci nuori o poco noti del Mediterraneo. $4^{\circ}$. Torino, 1857.

FINK (T. F.). De Amphibiorum Systemate Uropoetico. 8. Halla, 1817.

FINSCH (O.). Die Papageien, monographisch bearbeitet. 2 rols. $8^{\circ}$. London, 1861-68.

FINSCH (O.). Reise nach West-Sibirien im Jahre 1876. 8․ Berlin, 1879.

FiNSCH (O.). See 'Challenger.' 
FINSCH (O.) \& HARTLAUB (G.). Beiträge zur Fauna Centralpolynesiens. Ornithologie. 8o. Halle, 1867.

FINSCH (O.) \& HARTLAUB (G.). Die Vögel OstAfrikas. See Von der Decken's Reisen.

FISCHER (C.). Brevis Entozoorum seu Vermium Intestinalium Expositio.

$8^{\circ}$. Viennce, 1822 .

FISCHER (E. v. R.). Abbildungen zur Berichtigung und Ergänzung der Schmetterlingskunde.

4.. Leipzig, 1834.

FISCHER (G.). Anatomie der Maki.

4\%. Frankfurt am M., 1804.

FISCHER (G.). Zoognosia tabulis synopticis illustrata.

3 vols. $4^{\circ} \& 8^{\circ}$. Mosqua, 1813-14.

FISCHER (J.). De Pelvi Mammalium.

12. Tubinga, 1798.

FISCHER (J. B.). Synopsis Mammalium.

8.. Stuttgardice, 1829.

FISCHER (J. G.). Amphibiorum nudorum Neurologiæ specimen. $4^{\circ}$. Berolini, 1843.

FISCHER (J. G.). Ueber die Familie der Seeschlangen. $4^{\circ}$. Hamburg, 1855. 2nd edition. $4^{\circ}$. Hamburg, n. d.

FISCHER (J. G.). Neue oder wenig bekannte Reptilien. 8. Hamburg, 1879.

FISCHER (L. H.). Enumeratio Coleopterorum circa Friburgum Brisgoviæ indigenarum.

8. Friburgi Brisgovia, 1843.

FISCHER (L. H.). Orthoptera Europæa.

$4^{\circ}$. Lipsice, 1843.

FISCHER (P.). Spécies général et Iconographie des Coquilles Vivantes.

See Kiener (L. C.). Paris, 1880. 
FischeR (P.). See Survers \& Explorations: France.

FISCHER (S.). Ueber dio in der Umgebung von St. Petersburg vorkommenden Crustaceen aus der Ordnung der Brachiopoden und Entomostraceen.

$4^{\circ}$. St. Petersburg, 1847.

FISCHER (S.). Ergänzungen, Berichtigungen und Fortsetzung zu der Abhandlung ueber die in der Umgebung von St. Petersburg vorkommenden Crustaceen.

40. St. Petersburg, 1850.

EISCHER (S.). Abhandlung über das Genus Cypris.

$4^{\circ}$. St. Petersburg, 1851.

FISCHER (S.). See Surveys \& Explorations: Ressia.

FISCHER DE WALDHEIM (G.). Adversaria Zoologica. $4^{\circ}$. Mosqua, 1819.

FISCHER DE WALDHEIM (G.). Entomographia imperii Rossici. $4^{\circ}$. Mosqua, 1820-22.

FISCHER DE WALDHEIM $\left(G_{2}\right)$. Entomographie de la Russie. $4^{\circ}$. Moscou, 1851.

FISHERIES. (Austerdar.) De Walvischvangst, 1784. De Haringvisschery, 1786.

In 1 rol. $4^{\circ}$. Amsterctam, 1784-86.

FISHERIES. (BERLIN.) Beiträge zur Fischerei Statistik des Deutschen Reichs : bearbeitet von L. Wтттмаск.

40. Berlin, 1870-75.

FISHERIES. (BERLIx.) Internationale Fischerei Ausstellung, 1880 .

Axow. Catalog der Norwegischen Abtheilung.

$8^{\circ}$. Bergen, 1880.

, Die Fischereindustrie Norwegens von H. B.

$8^{\circ}$. Bergen, 1880.

" Katalog der Schweizerischen Betheiligung und Ichthyologische Mittheilungen aus der Schweiz.

8.. Leipzig, 1880. 
Aron. Special Catalogue of the Ningpo collection of exhibits.

$4^{\circ}$. Shanghai, 1880.

„Catalogus van Ingezonden Voorwerpen.

8․ Leiden, 1880.

FrIEDEL (E.). Führer durch die Fischerei-Abtheilung des Märkischen Provinzial-Museums der Stadtgemeinde Berlin. $\quad 8^{\circ}$. Berlin, 1880.

Knader (G.). Procédé pour l'épuration des eaux d'égouts d'établissements industriels.

[With an English translation.]

8. Halle sur S., 1880.

LUNDberg (R.). Schweden. I. Notizen über die Schwedischen Fischereien. II. Die Ostsee und Süsswasserfischereien. $\quad 8^{\circ}$. Stockholm, 1880.

Matsubara (S.). Special-Katalog für die Japanische Abtheilung. $8^{\circ}$. Berlin, 1880.

Trors (E. F.). Catalogo delle Dimostrazioni anatomiche del Museo del R. Istituto Veneto di Scienze, \&c.

$8^{\circ}$. Venezia, 1880.

FISHERIES. (BerLIN.) Amtliche Berichte über die Internationale Fischerei-Ausstellung zu Berlin, 1880.

I. Fischzucht. M. von dem BorNe, H. HAACK, K. Michaelis.

II. Seefischerei. Von M. Livdexan.

III. Seefischerei. Von A. MIEtzger.

IV. Fischereiproducte und Wasserthiere. Ton $\mathbf{H}$. DoHri, S. Friedländer und H. Nitsche.

V. Wissenschaftliche Abtheilung. Von J. Asurs, E. Friedel, O. Hermes, T. Holdefleiss, J . Magnus, E. Thorner, L. Wittmack.

8. Berlin, 1881.

FISHERIES. (Boston, U. S.) Reports of the Commissioners of Fisheries.

Third, Fourth, and Fifth Reports of the Commissioners of Fisheries, 1869-71. $\quad 8^{\circ}$. Boston, 1869-71.

Thirteenth and Fourteenth Reports of the Commissioners on Inland Fisheries. $8^{\circ}$. Boston, 1879-80. 
FISHERIES. (CoLdribs, U. S.) First and Second Annual Reports of the Ohio State Fish Commission.

$8^{\circ}$. Columbus, 1877-78.

FISHERIES. (Deblir.) Digest of the Principal Sections in the Acts of Parliament relating to the Irish Fisheries. Compiled by T. F. Brady.

8. Dublin, 1876.

An account of the present state of the Fisheries on the different parts of the coast of Ireland, for the year 1820.

8. Dublin, 1820-23.

Digest of Acts of Parliament and By-laws in force in Ireland for the Regulation of the Oyster Fisheries. By T. F. Brady.

8.. Dublin, 1881.

FISHERIES. (HavRE.) Rapport sur les Expositions internationales de Pêche de Boulogne-sur-mer, Arcachon et du Havre, 1866-68. Par J. L. Soubeyran.

8.. Paris, 1871.

FISHERIES. (Londox.) Report of the Committee appointed to enquire into the state of the Pilchard Fisheries. 1785-86. fol. London, 1786.

Report on Newfoundland Trade. (Parliamentary.) fol. London, 1817.

Report of Committee on Salmon Fisheries. (Parliamentary.)

fol. London, 1825.

Report of the Commissioners on Herring Fisheries, 1828-29. (Parliamentary.) fol. London, 1830.

Report on British Channel Fisheries. (Parliamentary.)

fol. London, 1833.

Abstract of Reports from the Commissioner of the Southern Whale Fishery Company.

8. London, 1850.

FISHERIES. (Loxpox.) International Fisheries Exhibition, 1883.

I. Catalogues. $8^{\circ} \& 4^{\circ}$.

America. United States of. (A.) Preliminary Catalogue and Synopsis of the Collections exhibited by the U.S. Fish Commission and by Special Exhibitors.

8. Washington, 1883. 
FISHERIES. (London.) International Fisheries Exhibition, 1883, continued.

Auerica. United States of. (B.) Collection of Economic Crustaceans, Worms, Echinoderms, and Sponges. By R.RathbUN. $8^{\circ}$. Washington, 1883.

" (C.) Catalogue of Aquatic and Fish-eating Birds exhibited by the U.S. National Museum. By Robert Ringway.

80. Washington, 1883.

China. Special Catalogue of the Chinese Collections of Exhibits. By R. HART. $\quad 4^{\circ}$. Shanghai, 1883.

India. Catalogue of the Exhibits in the Indian Section. By F. DAY. $\quad 8^{\circ}$. London, 1883.

New South Wales. Catalogue of the Exhibits in the New South Wales Court. By E. P. Ravrsay.

8. London, 1883.

Norwicr. Catalogue of a Collection of British Sea and Freshwater Fish and Fish-eating Birds exhibited by T. E. Gonv. sm. 8 ${ }^{\circ}$. Norwich, 1883.

St. Petersburg. Fishing and Hunting on Russian Waters. By O. Grimu. $8^{\circ}$. St. Petersburg, 1883.

Sweden. Special Catalogue of the Swedish Collection of Exhibits.

$8^{\circ}$. Stockholm, 1883.

Catalogue of Mr. Oscar Dickson's Collections from the Gottenborg Museum. 8\%. London, 1883.

II. Conference Papers. $8^{\circ}$. London, 1883.

Ansell (A. W.). On Trawling.

Bloomfield (J. C.). The Fisheries of Ireland.

Coвboln (T. Spencer). The Destruction of Fish by internal Parasites.

Corrish (T.). Crustaceans.

„ Mackerel and Pilchard Fisheries.

DAY (F.). On the Food of Fishes.

Duff (R. W.). Herring Fisheries of Scotland.

Edinburgin (H.R.H. the Duke of). Notes on the Sea Fisheries and Fishing Population of the United Kingdom.

Fryer (C. E.). A National Fishery Society.

Goode (G. Brown). The Fishery Industries of the United States. 
FISHERIES. (London.) International Fisheries Exhibition, 1883, continued.

Harding (C.). Molluses, Mussels, Whelks, \&c. used for Food or Bait.

Hoмe (D. M.). Salmon and Salmon Fisheries.

Howrtz (D.). Forest Protection and Tree Culture on Water Frontages.

Hubrecht (Prof.). Oyster Culture.

Huxuey (T. H.). Inaugural Address.

, Fish Diseases.

Joncus (L. Z.). The Fisheries of Canada.

Kent (W. S.). Artificial Culture of Lobsters.

Lankester (E. Ray). Scientific Results of the Exhibition.

Levi (Leone). Economic Condition of Fishermen.

Mainwaring (F. B. Massey). The Preservation of Fishlife in Rivers by the exclusion of town sewage.

Maitland (Sir J. R. G.). On the Culture of Salmonidce and the Acclimatisation of Fish.

Marston (R. B.). Coarse Fish Culture.

Moloney (Capt. C. A.). West-African Fisheries with particular reference to the Gold Coast Colony.

Mundahl (C. H.). Line Fishing.

Оковнг (Narinori). A Sketch of the Fisheries of Japan. Scotr (R. H.). Storm Warnings.

Shaw-Lefevre (Rt. Hon. G.). Principles of Fishery Legislation.

Sмiтн (F. A.). The Swedish Fisheries.

Sola (Lieut.-Col. F. G.). The basis for Legislation on Fishery questions.

„The Fisheries of Spain.

Temple (Capt.). Seal Fisheries.

Thompson (Sir H.). Fish as Food.

Wallem (F.M.). Notes on the Fish Supply of Norway.

WALPoLE (Spencer). Fish Transport and Fish Markets. 
FISHERIES. (London.) International Fisheries Exhibition, 1883, continued.

WAJSH (R. F.). Improved Facilities for the Capture,

Economic Transmission, and Distribution of SeaFishes.

WheELdon (J. P.). Freshwater Fishing in Great Britain other than Trout or Salmon.

III. HandBooks. $8^{\circ}$. London, 1883.

Adaus (W. M.). Fishermen and Fisheries of all countries.

Bertram (J. C.). The Unappreciated Fisher Folk.

$D_{A Y}(F$.$) Indian Fish and Fishing.$

, Fish Culture.

Fryer (C. E.). The Salmon Fisheries.

Holdsworth (E. W. H.). Apparatus for Fishing.

Howes (G. B.). Zoology and Food Fishes.

Knnt (W.S.). British Marine and Fresh-water Fishes.

LEe (H.). Sea Monsters unmasked.

„Sea Fables explained.

Manley (J. J.). Literature of Sea and River Fishing.

Pollock (F.). The Fishery Laws.

Robinson (P.). Fishes of Fancy.

Senror (W.). Angling in Great Britain.

Walpole (Spencer). The British Fish Trade.

Wheeldon (J. P.). Angling Clubs and Preservation Societies.

FISHERIES. (New South WALEs.) Report of the Royal Commission on the State and Prospect of Fisheries in the Colony. (Parliamentary.)

fol. London, 1880.

The Fisheries Act 1881.

8. Sydney, 1881.

FISHERIES. (St. Petersburg.) Investigation of the Condition of the Fisheries of Russia.

9 vols. $4^{\circ}$. St. Petersburg, 1860-75.

4 vols. Atlas, obl. fol. St. Petersburg, 1861-63-71-76. 
FISHERIES. (Trenron, U.S.) Fourth Annual Report of the Commissioners of Fisheries. $\quad 8^{\circ}$. Trenton, 1873.

FISHERIES. (Washington, U. S.) Report on the Sea Fisheries of New England, by S. F. Barrd.

8. Washington, 1873.

The History and Present Condition of the Fishery Industry. Prepared under the direction of S. F. BAird by G. B. Goode.

The Oyster-Industry, by E. Ingersoll. $4^{\circ}$. Washington, 1881.

The Seal-Islands of Alaska, by H. W. Elutotт. $4^{\circ}$. Washinaton, 1881.

FITCH (A.). An Essay upon the Wheat-Fly.

8. Albany, 1846.

FITCH (A.). The Hessian-Fly. $\quad 8^{\circ} . \quad$ Albany, 1847.

FITCH (A.). The American Currant-Moth (Abraxas ribearia).

8. Albany, 1848.

FITCH (A.). See Reports : U.S., Albany.

FITZINGER (L. J.). Neue Classification der Reptilien. $4^{\circ}$. Wien, 1826.

FITZINGER (L. J.). Ueber das System und die Charakteristik der natürlichen Familien der Vögel. $8^{\circ}$. Wien, $1856^{\circ}$.

FITZINGER (L. J.). Geschichte des Kais. Kön. HofNaturalien-Cabinetes. Abth. I. 8․ Wien, 1856. (Abth. II., III. Zool. Papers. $8^{\circ}$. Vol. 2.)

FITZINGER (L. J.). Ueber die Racen des zahmen Schafes. $8^{\circ}$. Wien, 1859-60.

FITZINGER (L. J.). Der Hund und seine Racen.

8․ Tübingen, 1876.

FITZROY (Capt.). See 'Beagle.'

FLEMING (J.). A History of British Animals.

$8^{\circ}$. Edinburgh, 1828. 2nd edition. $8^{\circ}$. London, 1842. 
FLEMING (J.). The Philosophy of Zoology.

2 vols. $8^{\circ}$. Edinburgh, 1832.

FLEMING (J.). Molluscous Animals, including Shell Fish. 8०. Edinburgh, 1837.

FLEMING and TIBBINS. See Dictionaries.

FLETCHER (W. J.)。 See Poole (W. F.)。

FLOR (G.). Rhynchotorum Livonicorum descriptio. Familia Prima. $\quad 8^{\circ}$. Dorpati Livon., 1856.

FLOR (G.). Die Rhynchoten Livlands.

2 vols. $8^{\circ}$. Dorpat, 1860-61.

FLOURENS (P.). Mémoires d'Anatomie et de Physiologie comparées.

$4^{\circ}$. Paris, 1844.

FLOURENS (P.). Buffon : histoire de ses travaux et de ses idées.

8. Paris, 1844.

FLOURENS (P.). Cuvier : histoire de ses travaux.

2nd edition. $8^{\circ}$. Paris, 1845.

FLOURENS (P.). Théorie expérimentale de la Formation des Os.

8.. Paris, 1847.

FLOURENS (P.). De l'Instinct et de l'Intelligence des Animaux. 4th edition. $8^{\circ}$. Paris, 1861.

FLOURENS (P.). De l'Unité de Composition et du Débat entre Cuvier et Geoffrox Saint-Hilaire.

8. Paris, 1865.

FLOURENS (P.). See Bufron (G.).

FLOWER (W. H.). Recent Memoirs on the Cetacea.

See Riy Societr.

fol. London, 1866.

FLOWER (W. H.). An Introduction to the Osteology of the Mammalia. post $8^{\circ}$. London, 1870. 2nd edition. post $8^{\circ}$. London, 1876. 
FLOWER (W. H.). Diagrams of the Nerves of the Human Body. 3rd edition. $4^{\circ}$. London, 1881.

FLOWER (W. H.). Fashion in Deformity, as illustrated in the customs of barbarous and civilized races.

('Nature' series.) 12. London, 1881.

FLOWER (W. H.). See Mrtsedm : College of Surgeods.

FLÜGEL (J. G.). See Dictinnaries.

FOCILLON (A.). Etudes sur les Insectes qui nuisent aux Colzas. $4^{\circ}$. Paris, 1852.

FOERSTER (A.). Beiträge zur Monographie der Pteromalinen, Nees. 4.. Aachen, 1841.

FOERSTER (A.). Hymenopterologische Studien. Heft I. Formicariae. $4^{\circ}$. Aachen, 1850. Heft II. Chatcidice und Proctotrupii. $4^{\circ}$. Aachen, 1856.

FOERSTER (A.). Monographie der Gattung Pezomachus. 8. Berlin, 1851.

FOERSTER (A.). Synoptische Uebersicht der Chalcidiæ und Proctotrupii. $\quad 4^{\circ}$. Aachen, 1856.

FOERSTER. Ueber den systematischen Werth des Flügelgeäders bei den Hymenopteren. $\quad 4^{\circ}$. Aachen, 1877.

FOHMANN (V.). Das Saugadersystem der Wirbelthiere. Heft I. Fische. fol. Heidelberg \& Leipzig, 1827.

FOLKES (M.). See Parsons (J.).

FONDOUCE (P. Cazalis de). Les Temps Préhistoriques dans le Sud-est de la France. L'homme dans la vallée inférieure du Gardon. $\quad 4^{\circ}$. Montpellier \& Paris, 1872.

FONTAINE (A. de la). Faune du Pays de Luxembourg, ou Manuel de Zoologie.

Parts I.-IV. $\quad 8^{\circ}$. Luxembourg, 1865-66-69-72.

FONTANA (F.). Abhandlung über das Viperngift.

2 rols. in $1.44^{\circ}$. Berlin, 1787. 
FORBES (E.). Malacologia Monensis.

8. Edinburgh, 1838.

FORBES (E.). A History of British Starfishes.

$8^{\circ}$. London, 1841.

FORBES (E.). A Monograph of the British Naked-eyed Medusce.

$4^{\circ}$. London, 1848.

See Ray Soctety.

FORBES (E.). On the Tertiary Fluvio-Marine formation of the Isle of Wight.

8. London, 1856.

FORBES (E.). The Natural History of the European Seas.

Edited by R. Godwin-A Usten.

$12^{\circ}$. London, 1859.

FORBES (E.) \& HANLEY (S.). A History of British Mollusca. 4 vols. $8^{\circ}$. London, 1853.

FORBES (E.). See 'Herald.'

FORBES (W. A.). See 'Challenger.'

FORBEiS (W. A.). See Garrod (A. H.).

FORD (L.). The Butterfly Collector's Vade-Mecum.

See JeRMry (L.). 3rd edition. $12^{\circ}$. London, 1836.

FORSKAL (P.). Descriptiones Animalium.

Ed. C. Niebuhr. $4^{\circ}$. Haunice, 1775.

FORSKÅ (P.). Icones Rerum Naturalium.

4. Haunice, 1776.

FORSTER (J. R.). Catalogue of British Insects.

8. Warrington, 1770.

FORSTER (J. R.). Noræ species Insectorum. Centuria I. 8. London, 1771.

FORSTER (J. R.). Catalogue of the Animals of North America.

8. London, 1771. 
FORSTER (J. R.). Enchiridion Historiæ Naturali inserviens.

8. Halce, 1788.

FORSTER (J. R.). Zoologia Indica. fol. Halle, 1795.

FORSTER (J. R.). Descriptiones Animalium.

Ed. H. Lichtenstein. $8^{\circ}$. Berolini, 1844.

FORSTER (J. R.). See Periodtcals : Londun. Willughby Society.

FORSTER (J. R.). See Latham (J.) and Davies (H.).

FORSTER (T.). Observations of the Natural History of Swallows. To which is added a General Catalogue of British Birds.

8. London, 1817.

FORSTER (T.). A Synoptical Catalogue of British Birds. 8. London, 1817.

FORSYTH (T. D.). See Henderson (G.) \& Home (A. O.).

FOSTER (M.). See KLeIN (E.).

FOUCHER D'OBSONVILLE. Essais Philosophiques sur les Mours de divers animaux étrangers, avec des observations relatives aux principes usages de plusieurs peuples. 8. Paris, 1783.

FOURCROY (A. F. de). Entomologia Parisiensis.

2 vols. $16^{\circ}$. Paris, 1785.

FOWLER (W.W.). See Matthews (A.) \& Fowler(W.W.).

FRANCK (L.). Handbuch der Anatomie der Hausthiere. 8.. Stuttgart, 1871.

FRANQUE (H.). Amicue Calvae Anatomiam descripsit tabulaque illustravit. fol. Berolini, 1847.

FRANQUE (H.). Afferuntur nonnulla ad Amiam Calvam accuratius cognoscendam.

fol. Berolini, 1847. 
FRANZIUS (W.). Historia Animalium.

18.. Amstelodami, 1665.

Another edition. $18^{\circ}$. Francofurti a. M., 1671.

FRANZIUS (W.). Historia Animalium, .... cum commentario et supplemento..... O Opera JoHannis Crpriant. $12^{\circ}$. Dresdae, 1687. Another edition. 4 vols. $4^{\circ}$. Francofurti \& Lipsiae, 1712.

FRASER (L.). Zoologia typica. fol. London, 1849.

FRASER (L.). Catalogue of the Knowsley Collections, belonging to the Earl of Derby. $\quad 8^{\circ}$. Knowsley, 1850.

FRAUENFELD (G. R. von). Neu aufgefundene Abbildung des Dronte und eines zweiten kurzflügeligen Vogels, wahrscheinlich des Poule rouge au bec de Bécasse der Maskarenen.

fol. Wien, 1868.

FRAUENFELD (G. R. von). See ' Novara.'

FREDOL (A.). Le Monde de la Mer. $\quad 8^{\circ}$. Paris, 1866.

FREMERY (N. C. de). Specimen zoologicum, de Casuario Novo-Hollandice. $\quad 8^{\circ}$. Trajecti ad Rhenum, 1819.

FREULER (J. J.). Monographia Caviæ Porcelli Zoologica. $4^{\circ}$. Goettingoe, 1820.

FREY (H.). Die Tineen und Pterophoren der Schweiz.

8. Züirich, 1856.

FREY (H.). Die Lepidopteren der Schweiz.

8.. Leipzig, 1880.

FREY (H.) \& LEUCKART (R.). Beiträge zur Kenntniss Wirbelloser Thiere. 4. Braunschweig, 1847.

FREYCINET (L. de). See ' Uranie' et ' Physicienne.'

FREYER (C. F.). Beiträge zur Geschichte europäischer Schmetterlinge. 3 vols. $16^{\circ}$. Augsburg, 1828-30

FREYER (C. F.). Neuere Beiträge zur Schmetterlingsizunde.

$4^{\circ}$. Augsourg, 1833-58. 
FREYER (C. F.). Die schädlichsten Schmetterlinge Deutschlands. $\quad 8^{\circ}$. Augsburg, 1856.

FREYER (H.). Fauna der in Krain bekannten Säugethiere, Vögel, Reptilien und Fische. $8^{\circ}$. Laibach, 1842.

FRIČ (A.). Die Wirbelthiere Böhems. $8^{\circ}$. Prag, 1870.

FRIČ (A.). Die Fluss-Fischerei in Böhmen und ihre Beziehungen zur künstlichen Fischzucht und zur Industrie.

8. Prag, 1871.

FRIDERICH (C. B.). Vollständige Naturgeschichte der Deutschen Zimmer-, Haus- und Jagdrögel.

3rd edition. $8^{\circ}$. Stuttgart, 1876.

FRIDERICI II. (Imperatoris) Reliqua Librorum de Arte Venandi cum avibus, et Albertus Jiagnus de Falconibus, $\& c$.

$12^{\circ}$. Augustce Vindelicorum, 1596.

FrIEDEL (E.). See Fisheries : Berlin.

FRIEDERICH (A.). Crania Germanica Hartagowensia.

$4^{\circ}$. Wernigerode, 1865.

FRIEDLÄNDER (C.). Microscopische Technik zum Gebrauch bei medicinischer und pathologisch-anatomischer Untersuchung.

8. Kassel und Berlin, 1882.

FRIEDLÄINDER (S.). See Fisheries: Berlin.

FRIELLE (H.). See Surveys \& Explorations : Norway.

FRIES (B. F.), EKSTRÖM (C. U.), \& SUNDEVALL(C. J.). Skandinaviens Fiskar. $\quad 4^{\circ}$. Stockholm, 1836-48.

FRISCH (J. L.). Beschreibung von allerley Insecten in Teutsch-Land, \&c. $\quad 4^{\circ}$. Berlin, 1730-38.

FRITSCH (A.). Naturgeschichte der Vögel Europa's. Text, 8․ Atlas, fol. Prag, 1871.

FRITSCH (A.). Zur Anatomie der Elephanten-Schildkröte (Testudo elephrantina). $\quad 4^{\circ}$. Prag, 1870. 
FRITSCH (G.). Untersuchungen über den feineren Bau des Fischgehirns.

$4^{\circ}$. Berlin, 1878.

FRIVALDSZKY(E.). Monographia Serpentum Hungariæ.

8. Pestini, 1823.

FRÖLICH (F.). Enumeratio Tortricum L. regno Wür-

tembergico.

8. Tubingce, 1828.

FROMENTAL (E. de). Etudes sur les Microzoaires, ou Infusoires proprement dits.

$4^{\circ}$. Paris, 1876.

FRYER (C. E.). See Frsheries : London.

FUESSLY (J. C.). Verzeichnis der ihm bekannten Schweitzerischen Inseckten. $\quad 4^{\circ}$. Zürich \& Winterthur, 1775.

FUESSLY (J. C.). Archiv der Insectengeschichte.

$4^{\circ}$. Zürich, 1781.

FUEssly (J. C.). See Periodicals : Zurich.

FUGGER (A. G. F.). De singulari clitoridis in Simiis generis Atelis magnitudine et conformatione.

$4^{\circ}$. Berolini, 1835.

FUHLROTT (C.). Der fossile Mensch aus dem Neanderthal.

8. Duisburg, 1865.

FULTON (R.). The Illustrated Book of Pigeons. (Edited by E. WRIGHT.)

$4^{\circ}$. London, n. d.

FUMOUZE (A.). De la Cantharide Officinale.

$4^{\circ}$. Paris, 1867.

FUNK (A.F.). De Salamandræ terrestris vita, evolutione, formatione tractatus.

fol. Berolini, 1827.

FUNKE (0.). See Hermann (L.). Handbuch der Physiologie.

FÜRBRINGER (M.). Die Knochen und Muskeln der Extremitäten bei den schlangenähnlichen Sauriern.

4. Leipzig, 1870. 
FÜRSTENBERG (M. H. F.), Die Krätzmilben der Menschen und Thiere.

$4^{\circ}$. Leipzig, 1861.

G.

GÄDE (H. M.). Beyträge zur Anatomie der Inseckten. Vorworte von C. H. Pfaff. Altona, 1815.

G̈̈DE (H. M.). Anatomie und Physiologie der Medusen. $8^{\circ}$. Berlin, 1816.

GÄDE (H. M.). Observationes quaedam de Insectorum Vermiumque structura. $4^{\circ}$. Kilice, 1817 .

GADEAU DE KERVILLE (H.). Les Insectes Phosphorescents. 8.. Rouen, 1881.

Gaimard (J. P.). See 'Uranie' et 'Physicienne.'

GALL (F. J.) \& SPURZHEIM (G.). Anatomie et Physiologie du système nerveux.

4 vols. and Atlas. Paris, 1810-19.

Gallwey. See Payne-Gallwey (R.).

GANIN (M.). Ueber die Embryonalhülle der Hymenopteren- und Lepidopteren-Embryonen.

$4^{\circ}$. St. Petersburg, 1869.

Garman (S. W.). See Museum : Harvard College.

GARROD (A. H.). The collected Scientific Papers of the late Alfred Henry Garrod, by W. A. Forbes.

8. London, 1881.

GARROD (A. H.). See 'Challinger.'

GASCO (F.). Intorno alla Balena presa in Taranto.

$4^{\circ}$. Napoli, 1878.

GASSIES (J. B.). Essai sur le Bulime tronqué.

8. Bordeaux, 1847. 
GASTALDI (B.). Lake Habitations and Pre-historic Remains of Northern and Central Italy. Translated by C. H. Chambers. $8^{\circ}$. London, 1865.

GATTERER (C. W. J.). Breviarium Zoologiæ. Pars I. Mammalia.

8. . Gottinga, 1780.

GAUBIL (J.). Catalogue Synonymique des Coléoptères d'Europe et d'Algerie.

$8^{\circ}$. Paris, 1849.

GAUCKLER (Ph.). Les Poissons d'eau douce et la Pisciculture.

8. Paris, 1881.

GAUDRY (A.). Mémoire sur les Pièces Solides des Stellerides.

4. Paris, 1852.

GAUTARD (V.). See Stierlin (G.).

GAVARD (H.). Traité complet d'Ostéologie. 3rd edition. 2 vols. $8^{\circ}$. Paris, 1805.

GAZZAIDI (D.) \& BATCHIERI (A.). Zoologia Moralo esposta in cento venti discorsi.

2 vols. fol. Firenze, 1843.

GEER (C. de). Mémoires pour servir à l'Histoire des Insectes.

7 vols. $4^{\circ}$. Stockholm, 1752-78.

GEER (C. de). Abhandlungen zur Geschichte der Inseckten. Aus dem Französischen übersetzt von J. A. E. GöTzE.

7 rols. $4^{\circ} . \quad$ Nurnberg, 1778-83.

GEER (C. de). Genera et Species Insectorum. Ed. A. J. RetziUs. 8. Lipsice, 1783.

GEGENBAUR (C.). Beiträge zur näheren Kenntniss der Schwimmpolypen (Siphonophoren). $\quad 4^{\circ}$. Leipzig, 1854.

GEGENBAUR (C.). Untersuchungen über Pteropoden und Heteropoden.

4. Leipzig, 1855.

GEGENBAUR (C.). De Animalium Plantarumque Regni Terminis et Differentiis.

4. Leipzig, 1860. 
GEGENBAUR (C.). Untersuchungen zur vergleichenden Anatomio der Wirbelsäule bei Amphibien und Reptilien.

4. Leipzig, 1862.

GEGENBAUR (C.). Untersuchungen zur vergleichenden Anatomie der Wirbelthiere.

Parts I.-III. 4. Leipzig, 1864-72.

GEGENBAUR (C.). Grundriss der vergleichenden Anatomie.

8. Leipzig, 1878.

GEGENBAUR (C.). Elements of Comparative Anatomy Translated by F. JefFrey BeLL and E. RAY LANkester.

8. London, 1878.

GEgENBAUR (C.). See Periodicals: Lempzig. Morphologisches Jahrbuch.

GEGENBAUR (C.). Lehrbuch der Anatomie des Menschen. 8. Leipzig, 1883.

GEGENBAUR (C.). See Rathre (H.).

GEHIN (J. B.). Catalogue des Coléoptères.

$8^{\circ}$. Metz, 1851-52.

GEHIN (J. B.). Revision des Poissons qui virent dans le Département de la Moselle.

8. Metz, 1868.

GEMMINGER (M.). Systematische Uebersicht der Käfer um München.

8. Jena, 1851.

GEMMINGER (M.) \& HAROLD (B. de). Catalogus Coleopterorum.

8. Monachii, 1868-76.

GENE (J.). Description de quelques espèces de la collection Zoologique de Turin. $4^{\circ}$. Turin, 1834.

GENE (J.). Descrizione di una Pecora, Capra Nubiana.

$4^{\circ}$. Torino, 1834.

GENE (J.). Considerazioni generali sulle Pecore e sulle Capre, con alcune speciali riflessioni sul muffione africano (Ovis tragelaphus, Auct.).

$4^{\circ}$. Torino, 1834. 
GENE (J.). De quibusdam Insectis Sardiniæ. Fasciculus I., II. $\quad 4^{\circ}$. Turin, 1837-38.

GENE (J.). Synopsis Reptilium Sardiniæ Indigenorum. $4^{\circ}$. Torino, 1839.

GENE (J.). Descrizione di un Nuovo Falcone di Sardegna (Falco eleanorae).

4. Torino, 1840.

GENTRY (T. G.). Illustrations of Nests and Eggs of Birds of the United States. $\quad 4^{\circ}$. Philadelphia, 1880-81.

GEOFFROY (E. L.). Histoire abregée des Insectes qui se trouvent aux environs de Paris.

2 vols. $4^{\circ}$. Paris, 1762.

GEOFFROY (E. L.). Histoire abrégée des Insectes rangés suivant un ordre methodique.

2 vols. in $4.4^{\circ}$. Paris, 1799.

GEOFFROY (S. L.). Abhandlungen von dem Gehörwerkzeuge des Menschen, der Amphibien und Fische.

8. Leipzig, 1780.

GEOFFROY SAINT-HILAIRE (E.). Systèmes dentaires des Mammifères et des Oiseaux.

8. . Paris, 1824.

GEOFFROY SAINT-HILAIRE (E.). Cours de l'Histoire Naturelle des Mammifères.

$8^{\circ}$. Paris, 1829.

GEOFFROY SAINT-HILAIRE (E.). Études Progressives d'un Naturaliste. 4. Paris, 1835.

GEOFFROY SAINT-HILAIRE (E.). Essais de Zoologie Générale.

$8^{\circ}$. Paris, 1841.

GEOFFROY SAINT-HILAIRE (I.) \& CUVIER (F.). Histoire Naturelle des Mammifères.

4 vols. fol. Paris, 1824.

GEOFFROY SAINT-HILAIRE (T.). Des Anomalies de l'Organisation.

2 vols. and Atlas. $8^{\circ}$. Bruxelles, 1837-8. 
GEOFFROY SAINT-HILAIRE (I.). Description des collections de Victor Jacquemont. Mammifères.

$4^{\circ} . \quad 1842-43$.

GEOFFROY SAINT-HILAIRE (I.). Catalogue méthodique des Mammifères. 8. Paris, 1851.

GEOFFROY SAINT-HILAIKE (I.). Histoire Naturelle générale des Règnes Organiques.

3 vols. $8^{\circ}$. Paris, 1854-62.

GEOFFROY SAINT-HILAIRE (I.). Acclimatation et Domestication des Animaux utiles.

4th edition. $8^{\circ}$. Paris, 1861.

GEOFFROY SAINT-HILAIRE (I.). See Avtelare (A.).

GEOFFROY SAINT-HilaiRE (I.). See Belaiger (C.).

GEOFFROY SAINT-HILAIRE (I.). See 'Vends.'

GERARD (C.). Essai d'une Faune historique des Mammifères sauvages de l'Alsace. $\quad 8^{\circ}$. Colmar, 1871.

GERARD (C.). See Surters and Explorations: U.S., Philadelphia.

GERARDIN (S.). Tableau Élémentaire d'Ornithologie. Texte, 2 vols., 80. Atlas, $4^{\circ}$. Paris, 1806.

GERBI (R.). Storia Naturale di un nuovo Insetto.

8. Firenze, 1794.

GERHARD (B.). Versuch einer Monographie der Europäischen Schmetterlingsarten.

$4^{\circ}$. Hamburg, 1853.

GERHARD (B.). Systematisches Verzeichniss der MacroLepidopteren von Nord-Amerika. $\quad 8^{\circ}$. Leipzig, 1878.

GERINI (G.). Storia Naturale degli Ucelli.

5 vols. fol. Firenze, 1767-76.

GERMAR (E. F.). Systematis glossatorum Prodromus sistens Bombycum species.

4.. Lipsice, 1811. 
GERMAR (E. F.). Reise nach Dalmatien und in das Gebiet von Ragusa. Leipzig \& Altenbery, 1817.

GERMAR (E. F.). Magazin der Entomologie.

4 vols. $8^{\circ}$. Halle, 1813-21.

GERMAR (E. F.). Insectorum species novæ.

8. Halce, 1824.

GERMaR (E. F.). See Periodicals : Halle \& Leipzig.

[GERRARD (E.).] Catalogue of the Bones of Mammalia in the British Museum.

See Musedm (British).

GERSTAECKER (A.). Rhipiphoridum dispositio systematica.

4\%. Berolini, 1855.

GERSTAECKER (A.). Bericht über die wissenschaftlichen Leistungen im Gebiete der Entomologie.

2 vols. $8^{\circ}$. Berlin, 1856-63.

GERSTAECKER (A.). Monographie der Endomychiden. 8. Leipzig, 1858.

GERSTAECKER (A.). Zur Morphologie der Orthoptera Amphibiotica.

$4^{\circ}$. Berlin, 1873.

GERSTAECKER (A.). Handbuch der Zoologie. Seo Cards (J. V.).

GERSTAECKER (A.). See Von DER Decken's Reisen.

GERSTFELDT (G.). Ueber einige zum Theil neue Arten Platoden, Anneliden, Myriapoden und Crustaceen Sibiriens. $4^{\circ}$. St. Petersburg, 1858.

GERSTFELDT (G.). Ueber die Flusskrebse Europa's.

$4^{\circ}$. St. Petersburg, 1859.

GERSTFELDT (G.). Ueber Land- und Süsswasser-Mollusken Sibiriens und des Amur-Gebietes.

$4^{\circ}$. St. Petersburg, 1859. 
GERVAIS (H.) \& BOULART (R.). Les Poissons.

3 vols. $8^{\circ}$. Paris, 1877.

GERVAIS (P.). Histoire naturelle des Mammifères.

4. Paris, 1854.

GERVAIS (P.). Ostéographie des Monotrèmes vivants et fossiles. $\quad$ Text, $4^{\circ}$. Plates, fol. Paris, 1877-78.

GERVAIS (P.). Nouvelles Planches Murales d'Histoire Naturelle. $8^{\circ} \&$ fol. Paris, 1880.

GERVAIS (P.). See Beneden (P. J. van).

GERVAIS (P.). See ' Favorite.'

GERVAIS (P.). See Periodicals : Paris.

GESNER (C.). Historia Animalium.

4 vols. fol. Tiguri, 1551-85. 2nd edition. 3 vols. $4^{\circ}$. Francofurti, 1620-21.

GESNER (C.). Icones Animalium. $\quad 4^{\circ}$. Zurich, 1553.

GESNER (C.). Thierbuch.

4. Heidelberg, 1606.

GESNER (C.). See Dubratius (J.).

GEUBEL (H. K.). Neuere Beiträge zur Zoologie.

8. Frankfurt am Main, 1846.

GEVENS (N. G.). See Bachiant (F.).

GIBBES (L. R.). On the Carcinological Collections of Natural History in the United States.

80. Charleston, S. C., 1850-58.

GIBBES (R. W.). Monograph of the Fossil Squalida of the United States.

$4^{\circ}$. Philadelphia, 1848.

GIBBES (R, W.). Memoir on Mosasaurus.

40. Philadelphia, 1849.

GIEBEL (C. G.). Die Säugethiere. $\quad 8^{\circ}$. Leipzig, 1859. 
GIEBEL (C. G.). Thesaurus Ornithologiæ.

8.. Leipzig, 1872.

GIEBEL (C. G.). Insecta epizoa. fol. Leipzig, 1874.

GIEBEL (C. G.). Odontographie. $\quad 4^{\circ} . \quad$ Leipzig, 1855.

GIGLIOLI (E. H.). Ricerche intorno alla Distribuzione Geografica Generale Corologia degli Animali Vertebrati.

8. Roma, 1873.

GIGLIOLI (E. H.). Iconographia dell' Avifauna Italica. fol. Prato, 1879-83.

GIGLIOLI (E. H.). Elenco dei Mammiferi, degli Uccelli e dei Rettili.

$8^{\circ}$. Roma, 1881.

GILbERT (C. H.). See Periodicals : Washington.

GILIJ (P. A.). Agri Romani Historia Naturalis. Pars prima, Ornithologia.

$8^{\circ}$. Roma, 1781.

GILL (T.). See Suithisonian Miscellaneods Collections.

GILLMEISTER (C. J. F.). Trichopterygia.

8. Nürnberg, 1845.

GILTAY (C. MI.). Commentatio de Esoce Zucio.

$4^{\circ}$. Lugduni Batavorum, 1832.

GINANNI (G.). Delle uovi degli Ucelli, Libro primo.

$4^{\circ}$. Venezia, 1737.

GIOVANNI (Z.). See Periodicals : Milan.

GIOVIO (P.). De Pesci Romani.

sm. $4^{\circ}$. Venetia, 1560.

GIRARD (C.). See BaIrd (S. F.).

GIRAUD-TEULON (F.). Principes de Mécanique Animale.

$8^{\circ}$. Paris, 1858. 
GIRDWOYN (M.). Anatomie et Physiologie de l'Abeille. $4^{\circ}$. Paris, 1876.

GIRGENSOHN (O. G. L.). Anatomie und Physiologie des Fischnervensystems. $4^{\circ}$. St. Petersburg, 1846.

GIRTANNER (A.). Der Alpensteinbock (Capra Ibex, L.). $8^{\circ}$. Trier, 1878.

GISTL (J.). Enumeratio Coleopterorum Agri Monacensis. 8. . Augustoe Vindelicorum, 1831.

GISTL (J.). Faunus : Zeitschrift für Zoologie und vergleichende Anatomie.

2 vols. in 1. $8^{\circ}$. München, 1832-35.

GISTL (J.). Beschreibung des Skeletes des dreistreifigen Nachtäffers (Nyctipithecus trivirgatus).

8. Leipzig, 1836.

GISTL (J.). Systema Insectorum. 8º Berno, 1837-39.

GISTL (J.). Lexicon der Entomologischen Welt, der Carcinologischen und Arachnologischen.

8. Stuttgart, $18+6$.

GLIDDON (G. R.). See Notт (J. C.).

GLOGER (G. L.). Schlesiens Wirbelthier Fauna.

8. Breslau, 1833.

GLOGER (C. L.). Das Abändern der Vögel durch Einfluss des Klima's. $\quad 8^{\circ}$. Breslau, 1833.

GLOGER (C. W. L.). Gemeinnütziges Hand- u. Hilfsbuch der Naturgeschichte. $\quad 8^{\circ}$. Breslau, 1842.

GMELIN (J. F.). Systema Naturæ.

8 vols. $8^{\circ}$. Lugduni, 1788-96.

GMELIN (J. G.). J. G. G. . . . reliquias quæ supersunt commercii epistolici cum Carolo Linnaeo, Alberto Hallero, Guilielmo Stellero et al., publicandas curavit G. H. T. Pliminivger.

$8^{\circ}$. Stuttgartice, 1861. 
GMLLIN (G. A.). Ueber den Winter-Schlaf.

8'. '1 ̈̈bingen, 1839.

GODART (J. B.) \& DUPONCHEL (P. A. J.). Histoire naturelle des Lépidoptères ou Papillons de la France.

Vols. I.-X. Suppl., vol. I. $8^{\circ}$. Paris, 1821-32.

GODMAN (F. D.). Natural History of the Azores.

$8^{\circ}$. London, 1870.

GODMaN (F. D.). See Biologia Centrali-Americana.

GODMAN (J. D.). AAmerican Natural History.

2 vols. $8^{\circ}$. Philadelphia, 1826.

GODWLN (D. A.). Zoologie de la Lorraine.

8. Paris \& Nancy, 1863.

GODWIN-AUSTEN (H. H.). Land and Freshwater Mollusca of India.

Parts I.-III. Text, $8^{\circ}$. Plates, $4^{\circ}$. London, 1882-83. [In progress.]

GODWIN-AUSTEN (R.). See Forbes (E.).

GOEDARTIUS (J.). Metamorphosis et Historia naturalis Insectorum. 12. Medioburgi, 1662.

GOEDARTIUS (J.). De Insectis. (Appendix by M. LISTER.) $12^{\circ}$. Londini, 1685.

GOEDARTIUS (J.). Metamorphoses naturelles ou Histoire des Insectes.

12. Amsterdam, 1700.

GOETTE (A.). Die Entwickelungsgeschichte der Unke (Bombinator igneus).

Text, $8^{\circ}$. Atlas, fol. Leipzig, 1874-75.

GOETTE (A.). Ueber Entwickelung und Regeneration der Gliedmassenskelets der Molche. $\quad 4^{\circ}$. Leipzig, 1879.

GOETTE (A.). Abhandlungen zur Entwickelungsgeschichte der Thiere. Heft I. $8^{\circ}$. Leipziy, 1882. 
GOEZE (J. A. E.). Entomologische Beyträge.

4 vols. $8^{\circ}$. Leipzig, 1777.

GOEZE (J. A. E.). Versuch einer Naturgeschichte der Eingeweidewürmer thierischer Körper.

$4^{\circ}$. Blankenburg, 1782 .

GOEZE (J. A. E.). See Trembley (A.).

GOEZE (J. U. C.) \& DONNDORF (J. A.). Europäische Fauna, oder Naturgeschichte der Europäischen Thiere.

9 vols. in 10. Leipzig, 1791-1803.

GOLDBERG (O.T.). Helminthum dispositio systematica. $4^{\circ}$. Berolini, 1855.

GOLDFUSS (G. A.). Ueber die Entwickelungsstufen des Thieres. 8. Nürnberg, 1817.

GOLDFUSS (G. A.). Handbuch der Zoologie.

2 vols. $8^{\circ}$. Nürnberg, 1820.

GOLDFUSS (A.). Ausführliche Erläuterung des Naturhistorischen Atlasses.

3 vols. Text and Atlas. $4^{\circ}$. Düsseldorf, 1826.

GOLDSMITH (0.). History of the Earth and Animated Nature. 6 vols. $8^{\circ}$. London, 1816.

GOODE (G. B.). See Fishertes: Washington.

GOODE (G. B.). Game Fishes of the United States. 10 parts. obl. fol. New York, 1878-80.

Goodhart (J. F.). See Musedm : College of Surgeons.

GOODSIR (J.). See Periodicals : Edinburgh.

GORSKI (C.). Ueber das Becken der Saurier. 4.. Mitau und Leipzig, 1852.

GORSKI (S. B.). Analecta ad Entomographiam provinciarum occidentali-meridionalium Imperii Rossici.

8. Berlin, 1852. 
GORY (H.). See Laporte (F. L. de) \& Gory (H.).

GORY (H.) \& PERCHERON (A.). Monographie des Cétoines. 8. Paris, 1833.

GOSSE (P. H.). The Canadian Naturalist. sm. $8^{\circ}$. London, 1840.

GOSSE (P. H.). The Birds of Jamaica.

$8^{\circ}$. London, 1847.

GOSSE (P. H.). Illustrations of the Birds of Jamaica.

$4^{\circ}$. London, 1849.

GOSSE (P. H.). Natural History-Birds.

12. London, 1849.

GOSSE (P. H.). The Ocean.

8. London, 1849.

GOSSE (P. H.). A Naturalist's Rambles on the Devonshire Coast.

8. . London, 1853.

GOSSE (P. H.). Natural History:-Mollusca.

12. London, 1854.

GOSSE (P. H.). A Manual of Marine Zoology.

2 parts. $8^{\circ}$. London, 1855-56.

GOSSE (P. H.). The Aquarium.

2nd edition. $8^{\circ} . \quad$ London, 1856.

GOSSE (P. H.). Tenby : a Sea-side Holiday.

sm. 8. London, 1856.

GOSSE (P. H.). Omphalos: an Attempt to untie the Geological Knot. $\quad 8^{\circ}$. London, 1857.

GOSSE (P. H.). Life in its lower, intermediate, and higher forms.

8.. London, 1857.

GOSSE (P. H.). A History of the British Sea-Anemones and Corals.

8․ London, 1860. 
GOSSE (P. H.). A Year at the Shore.

sm. $4^{\circ}$. London, 1865.

GOTHWALDT (C.). Physikalisch-anatomische Bemerkungen über die Schildkröten. $\quad 4^{\circ}$. Nürnberg, 1781.

GOTHWALDT (C.). Physikalisch-anatomische Bemerkungen über den Biber. $\quad 4^{\circ}$. Nürnberg, 1782.

GÖTZE (J. A.). See GEER (C. de).

GOUAN (A.). Histoire des Poissons.

$4^{\circ}$. Strasburg, 1770.

GOUAN (A.). Geschichte der Fische. [Trans. from the Latin by K. F. von Meidinger.] $\quad 8^{\circ}$. Wien, 1781.

GOULD (A. A.). Otia Conchologica. $8^{\circ}$. Boston, 1862.

GOULD (A. A.) \& BINNEY (W. G.). Report on the Invertebrata of Massachusetts.

2nd edition. $8^{\circ}$. Boston, 1870.

GOULD (A. A.). See Brrvey (A.).

GOULD (A. A.). Mollusca.

Text, $4^{\circ}$. Plates, fol. Philadelphia, 1852. See Survers ayd Explorations: U.S., Philadelphia.

GOULD (J.). A Century of Birds from the Himalaya Mountains.

fol. London, 1831.

GOULD (J.). Icones Arium.

fol. London, 1837.

GOULD (J.). A Monograph of the Ramphastidce.

2 vols. fol. London, 1834-38.

GOULD (J.). The Birds of Europe.

5 vols. fol. London, 1837.

GOULD (J.). A Monograph of the Trogonida.

fol. London, 1838. 
GOULD (J.). Synopsis of the Birds of Australia.

8. London, 1837-38.

GOULD (J.). An Introduction to the Birds of Australia. 8.. London, 1848.

GOULD (J.). A Monograph of the Odontophorince.

fol. London, 1850.

GOULD (J.). Monograph of the Trochilida, or family of Humming-Birds.

5 vols. fol. London, 1849-61. Supplement. fol. London, 1880.

GOULD (J.). An Introduction to the Trochilida, or family of Humming-Birds.

8.. London, 1861.

GOULD (J.). The Birds of Asia. fol. London, 1850-79.

GOULD (J.). The Birds of Australia.

7 vols. and Supplement. fol. London, 1851-69.

GOULD (J.). The Birds of Great Britain.

5 vols. fol. London, 1862-73.

GOULD (J.). The Mammals of Australia.

fol. London, 1863.

GOULD (J.). A Handbook of the Birds of Australia.

2 vols. roy. $8^{\circ}$. London, 1865.

GOULD (J.). The Birds of New Guinea.

fol. London, 1875-83.

GOULD (J.). See 'Beagle.'

GOULD (J.). See Martin (W. C. L.).

GOULD (W.). An account of English Ants.

8. London, 1747.

GOUPIL (C. J.). Histoire des Mollusques.

$12^{\circ}$. Paris, 1835.

GRAAF (H. W. de). Over den Bouw der Geslactsorganen bij de Phalangiden. $\quad 4^{\circ}$. Leiden, 1882. 
GRABER (V.). Beitrag zur Histologie der Stachelbäuter. 8. . Graz, 1872.

GRABER (V.). Ueber das Verdauungs-System des Kiefern Prachtkäfers, Chalcophora marina, Linn.

8․ Graz, 1874.

GRABER (V.). Die Naturkräfte. Die Insekten.

12․ München, 1877-79.

GRAELLS (M. P.). Catálogo de los Moluscos Terestres y de aqua dulce observados en España. $8^{\circ}$. Madrid, 1846.

GRAELLS (M. P.). Memorias de la Comision del Mapa Geológico de España. Parte Zoológica. Coleópteros.

$4^{\circ}$. Madrid, 1858.

GRAELLS (M. P.). Exploracion científica de las costas del departamento maritimo de Ferrol. $8^{\circ}$. Madrid, 1870.

GRAEFFE (E.). Reisen im Innern der Insel Viti-Levu.

4. Zürich, 1868.

GRAFF (L.). Das Genus Myzostoma.

4. Leipzig, 1877.

GRAFF (L. von). Monographie der Turbellarien. I. Rhobdocoelida. Text und Atlas. $4^{\circ}$. Leipzig, 1882.

GRAESSE (J. G. T.). Orbis Latinus.

8. Dresden, 1861.

GRAESSNER (F.). Die Vögel von Mittel-Europa und ihre Eier. 3rd edition. $4^{\circ}$. Dresden, n. d.

GRAHAM (F.). Visits to the Zoological Gardens.

$8^{\circ}$. London, $\mathrm{n}$. d.

GRANDIDIER (A.). Histoire Physique, Naturelle et Politique de Madagascar.

Mammifères par A. Milne-Edwards et A. Grandidier.

2 vols. $4^{\circ}$. Paris, 1875.

Oiseaux par A. Milne-Edwards et A. Grandidier.

4 vols. $4^{\circ}$. Paris, 1879-82. 
GRANT (R. E.). Outlines of Comparative Anatomy.

8. London, 1841.

GRAS (A. le). Description des Oursins fossiles du département de l'Isère.

8. Paris, 1848.

GRASSI (B.). See FAtra und Flora des Golfes ron Neapel.

GRATIOLET (L. P.) \& ALIX(E.). Recherches sur l'Anatomie de l'Hippopotame. $4^{\circ}$. Paris, 1867.

GRAVENHORST (J. L. C.). Coleoptera Microptera Brunswicensia necnon Exoticorum. 8. Brunswigce, 1802.

GRAVENHORST (J. L. C.). Monographia Coleopterorum Micropterorum.

8\%. Gottingae, 1806.

GRA VENHORST (J. L. C.). Monographia Ichneumonum pedestrium. $8^{\circ}$. Lipsice, 1815.

GRAVENHORST (J. L. C.). Ichneumonologia Europæa. 3 vols. $8^{\circ}$. Vratislavia, 1829.

GRA VENHORST (J. L. C.). Beiträge zur Entomologie. 8. Breslau, 1829. Another copy. See Periodicals : Breslad.

GRAVENHORST (J. L. C.). Reptilia Musei Zoologici Vratislarensis recensita et descripta. $\quad 4^{\circ}$. Lipsice, 1829.

GRAVENHORST (J. L. C.). Tergestina.

8. Brestau, 1831.

GRAVENHORST (J. L. C.). Über Phrynosoma orbicularis, Trapelus hispidus, .... und Chamceleopsis Hermandesii.

$4^{\circ}$. Bonn, 1833.

GRAVENHORST (J. L. C.). Beiträge zur genauern Kenntniss einiger Eidechsengattungen. $4^{\circ}$. Bonn, 1837. 
GRAVENHORST (J. L. C.). Ueber die im zoologischen Museum der Universität Breslau befindlichen Wirtelschleichen und andere Reptilien \&c.

$4^{\mathrm{o}}$. Breslcu, 1847.

GRAVES (G.). British Ornithology.

3 vols. $8^{\circ}$. London, 1811. 2nd edition. 3 vols. $8^{\circ}$. London, 1821.

GRAVES (G.). The Naturalist's Pocket Book.

8. London, 1818.

GRAY (E. W.). Catalogue of Shells. Part I. Univalves. 8․ MS., 1791.

GRAY (G. R.). Synopsis of the species of Insects belonging to the Family Phasmidce. $\quad 8^{\circ}$. London, 1835.

GRAY (G. R.). Descriptions and Figures of some new Lepidopterous Insects, chiefly from Nepal. [With a set of proof-sheets.]

8. London, 1846.

GRAY (G. R.). List of Birds in the British Museum.

$12^{\circ}$. London, $1848-68$.

GRAY (G. R.). List of British Animals in the British Museum. Birds.

$12^{\circ}$. London, 1850.

GRAY (G. R.). List of British Animals in the British Museum. Eggs of British Birds. 12 . London, 1852.

GRAY (G. R.). Catalogue of Lepidopterous Insects in the British Museum. Part I. Papilionidae.

$4^{\circ}$. London, 1852.

GRAY (G. R.). List of Lepidopterous Insects in the British Museum. Part I. Papilionidae.

$12^{\circ}$. London, 1856.

GRAY (G. R.). Birds of the Tropical Islands of the Pacific Ucean. (Brit. Mus.) $\quad 8^{\circ}$. London, 1859.

GRAY (G. R.). Catalogue of British Birds in the British Museum.

8. London, 1863. 
GRAY (G. R.). Hand-list of Genera and Species of Birds. 8. London, 1869-71.

GRAY (G. R.). See 'ErebUs' and 'Terror.'

GRAY (G. R.) \& MITCHELL (D. W.). The Genera of Birds. 3 vols. fol. London, 1844-49.

GRAY (J. E.). Spicilegia Zoologica.

$4^{\circ}$. London, 1828-30.

GRAY (J. E.). Illustrations of Indian Zoology.

2 vols. fol. London, 1830-32.

GRAY (J.E.). Ornithological Miscellany. Raptorial Birds. $4^{\circ}$. London, 1830 ?

GRAY (J. E.). Synopsis Reptilium. 8º London, 1831.

GRAY (J. E.). Zoological Miscellany.

$8^{\circ}$. London, 1831-44.

GRAY (J. E.). A Manual of Land and Freshwater Shells of the British Islands.

8. London, 1840.

GRAY (J. E.). List of Mammalia in the British Museum. $12^{\circ}$. London, 1843.

GRAY (J. E.). Catalogue of the Tortoises, Crocodiles, and Amphisbænians in the British Museum.

$12^{\circ}$. London, 1844.

GRAY (J. E.). Catalogue of Lizards in the British Museum.

$12^{\circ}$. London, 1845.

GRAY (J. E.). Gleanings from the Menagerie and Aviary at Knowsley Hall. fol. Knowsley, 1846-50.

GRAY (J. E.). A List of the Genera of recent Mollusca. 8. London, 1847.

GRAY (J. E.). List of the Osteological Specimens in the British Museum.

12\%. London, 1847. 
GRAY (J. E.). Catalogue of Snakes in the British Museum.

$12^{\circ}$. London, 1849.

GRAY (J. E.). Catalogue of the Mollusca in the British Museum.

$12^{\circ}$. London, 1849-50-53.

GRAY (J. E.). Catalogue of Mammalia.

Part I. Cetacea. 12\%. London, 1850.

Part II. Seals. $12^{\circ}$. London, 1850.

Part III. Ungulata Furcipeda. $12^{\circ}$. London, 1852.

GRAY (J.E.). Catalogue of Amphibia in the British Museum. Part II. Batrachia Gradientia, \&c.

$12^{\circ}$. London, 1850.

GRAY (J. E.). Catalogue of the Bivalve Mollusca in the British Museum.

$12^{\circ}$. London, 1850.

GRAY (J. E.). List of Fish in the British Museum. Part I. Chondropterygii.

12․ London, 1851.

GRAY (J. E.). Catalogue of Shield Reptiles in the British Museum. Part I. Testudinata (Tortoises).

$4^{\circ}$. London, 1855.

Supplement to same. $4^{\circ}$. London, 1870.

Appendix to same. $4^{\circ}$. London, 1872.

Part II. Emydosaurians, Rhynchocephalia, and Amphisbænians.

$4^{\circ} .1872$.

GRAY (J. E.). List of the Mollusca in the British Museum. Part I. Volutidce. $12^{\circ}$. London, 1855. Part II. Olividce. $12^{\circ}$. London, 1865.

GRAY (J. E.). Guide to the Systematic Distribution of Mollusca in the British Museum. $12^{\circ}$. London, 1857.

GRAY (J. E.). Manual of the Land and Freshwater Shells of the British Islands.

$8^{\circ}$. London, 1857.

GRAY (J. E.). Catalogue of Seals and Whales in the British Iuseum. Second edition. $8^{\circ}$. London, 1866. Supplement. $8^{\circ}$. London, 1871.

GRAY (J. E.). Catalogue of Carnivora, Pachydermata, and Edentata in the British Museum. 8 ${ }^{\circ}$ London, 1869. 
GRAY (J. E.). Catalogue of Sea-Pens, or Pennatulariidoe, in the British Museum.

8. London, 1870.

GRAY (J.E.). Catalogue of Lithophytes, or Stony Corals, in the British Museum.

$8^{\circ}$. London, 1870.

GRAY (J. E.). Catalogue of Monkeys, Lemurs, and Fruit-eating Bats in the British Museum.

8․ London, 1870.

GRAY (J. E.). Catalogue of Ruminant Mammalia (Pecora, Linnæus) in the British Museum.

8. London, 1872.

GRAY (J. E.). Hand-list of the Specimens of Shield Reptiles in the British Museum.

80. London, 1873.

GRAY (J. E.). Hand-list of the Edentate, Thick-skinned, and Ruminant Mammals in the British Museum.

8. London, 1873.

GRAY (J. E.). Hand-list of Seals, Morses, Sea-lions, and Sea-bears in the British Museum. $8^{\circ}$. London, 1874.

GRAY (J. E.). See 'Blossom.'

GRAY (J. E.). See 'ErebUs' and 'Terror.'

GRAY (J. E.). See 'Samarang.'

GRAY (J. E.). See Sowerby (J. de C.) \& Lear (E.).

GRAY (J. E.). List of the Books, Memoirs, \&c.

8. London, 1875.

GRAY (J. E. \& G. R.). Catalogue of Mammalia and Birds of New Guinea in the British Museum.

12․ London, 1859.

GRAY (J. E. \& G. R.). Catalogue of the Mammalia and Birds of Nepal and Thibet. 12. London, 1846.

GRAY (J. E.) \& PFEIFFER (L.). Catalogue of Phaneropneumona in the British Museum.

$12^{\circ}$. London, 1852. 
GRAY (J. E.) \& PFEIFFER (L.). Catalogue of Pulmonata, or Air-breathing Mollusca, in the British Museum.

12\%. London, 1855.

GRAY (M. E.). Figures of Molluscous Animals. 5 vols. $8^{\circ}$. London, 1859.

GRAY (R.). The Birds of the West of Scotland, including the Outer Hebrides.

8. Glasgow, 1871.

GRAY (R.) \& ANDERSON (F.). The Birds of Ayrshire and Wigtonshire.

8. Glasgow, 1869.

GREDLER (P. V. M.). Die Käfer von Tirol.

2 vols. \& supp. $8^{\circ}$. Bozen, 1863-66.

GREENE (J. R.). Manual of the Protozoa.

$12^{\circ}$. London, 1871.

GRENACHER (H.). Untersuchung über das Sehorgan der Arthropoden insbesondere der Spinnen, Insecten und Crustaceen.

$4^{\circ}$. Göttingen, 1879.

GRENIER (A.). Catalogue des Coléoptères de France.

8.. Paris, 1863.

GREY (W.). Catalogus Insectorum.

8. MS. Petropoli, 1866.

GRIFFITH (E.). General and particular Descriptions of the Vertebrated Animals. Order Quadrumana.

8. London, 1821.

GRIFFITH (E.). The Animal Kingdom.

See Cuvier (G.).

16 vols. $8^{\circ}$. London, 1827-33.

GRIFFITH (J. W.). See MÜLLer (J.).

GRIFFITH (R.) \& M'COY (F.). Silurian Fossils of Ireland. 4. Dublin, 1846.

GRILLO-TALPA. Seu MI. (G.). 
GRIMM (O.). Die ungeschlechtliche Fortpflanzung einer Chironomus-Art. $4^{\circ}$. St. Petersburg, 1870.

GRIMII (0.). Beiträge zur Lehre von der Fortpflanzung und Entwickelung der Arthropoden.

$4^{\circ}$. St. Petersburg, 1871.

GRIMI (O.). Fauna of the Caspian Sea.

Parts I., II. $8^{\circ}$. St. Petersburg, 1876-77.

GRIMIM (0.). See Fisheries: London. International Fisheries Exhibition, 1883.

GRIMIMER (K. H. B.). Steiermark's Coleoptern mit Einhundert sechs neu beschriebenen Species. 8*. Gratz, 1841.

GROGNOT (Ainé). Mollusques Testacés du département de Saone-et-Loire.

8. Autun, 1863.

GRONOVIUS (A.). See Autanus.

GRONOVIUS (L. T.). Museum Ichthyologicum.

fol. Lugduni, 1754.

GRONOVIUS (L. T.). Zoophylacium Gronovianum.

fol. Lugduni, 1763-81.

GRONOW (L. T.). Catalogue of Fish collected and described by L. T. Gronow, in the British Museum.

$8^{\circ}$. London, 1854.

GROSHANS (G. P. F.). Specimen anatomico-physiologicum de systemate-uropoietico.

8. . Lugduni Batavorum, 1837.

GROTE (A.). See Hewitson (W. C.).

GROTE (A. R.). New Check-List of North-American Moths.

8․ New York, 1882.

GROTE (A. R.). The Noctuidce of North America with "A Colony of Butterflies."

$8^{\circ}$. London, 1882.

Grote (A. R.). See Periodicals: Buffalo. 
GRUBE (A. E.). De Pleione carunculatc. Dissertatio Zootomica. $4^{\circ}$. Regiomontii Prussorum, 1837.

GRUBE (A. E.). Zur Anatomie und Physiologie der Kiemenwürmer. $\quad 4^{\circ}$. Königsberg, 1838.

GRUBE (A. E.). Untersuchungen über die Entwicklung der Anneliden.

$4^{\circ}$. Königsberg, 1844.

GRUBE (A. E.). Actinien, Echinodermen und Würmer des Adriatischen- und Mittelmeers. $4^{\circ}$. Königsberg, 1846.

GRUBE (A. E.). Die Familien der Anneliden mit Angabe ihrer Gattungen und Arten.

8. Berlin, 1851.

GRUBE (A. E.). Ein Ausflug nach Triest und den Quarnes. 8. Berlin, 1861.

GRUBE (A. E.). Die Insel Sussin und ihre Meeresfauna. 8. Breslau, 1864.

GRUBE (E.). Annulata Semperiana. Annelidenfauna der Philippinen. $\quad 4^{\circ}$. St. Petersburg, 1878.

GRUBE (E.). See 'Novara.'

GRUBE (E.). See Survers \& Explorations: Russia.

GRUBER (W.). Menschliche Missbildung mit Spaltungen an der Rücken- und Bauchfläche und noch anderen Deformitäten.

4․ St. Petersburg, 1849.

GRUBER (W.). Abhandlungen aus der menschlichen und vergleichenden Anatomie. $4^{\circ}$. St. Petersburg, 1852.

GRUBER (W.). Anatomie der Eingeweide des Leoparden (Felis leopardus) mit vergleichenden Bemerkungen über andere Felis-Arten. $\quad 4^{\circ}$. St. Petersburg, 1855.

GRUBER (W.). Monographie des canalis supracondyloideus humeri und der processus supracondyloidei humeri et femoris der Säugethiere und des Menschen.

$4^{\circ}$. St. Petersburg, 1856. 
GRUBER (W.). Die Musculi subscapularies und die neuen supernumerären Schultermuskeln des Menschen.

$4^{\text {o. }}$ St. Petersburg, 1857.

GRUBER (W.). Die bursæ mucosæ der spatia intermetacarpo-phalangea et intermetatarso-phalangea.

$4^{\circ}$. St. Petersburg, 1858.

GRUBER (W.). Ueber den zeitlichen Hermaphroditismus eines 22-jährigen Menschen. $\quad 4^{\circ}$. St. Petersburg, 1859.

GRUBER (W.). Menschliches Analogon der thierischen vagina nervi trigemini ossea am Felsenbeine.

$4^{\circ}$. St. Petersburg, 1859.

GRUBER(W.). Missbildungen. Erste Sammlung.

$4^{\circ}$. St. Petersburg, 1859.

GRUBER (W.). Beiträge zur Anatomie des Keilbeines und Schläfenbeines. 4\%. St. Petersburg, 1859.

GRUBER (W.). Die Oberschulterhakenschleimbeutel (bursæ mucosæ supracoracoideæ). $4^{\circ}$. St. Petersburg, 1861.

GRUBER (W.). Ueber den sinus communis und die valvulæ der venæ cardiacæ, und über die Duplicität der vena cava superior bei dem Menschen und den Säugethieren.

$4^{\circ}$. St. Petersburg, 1864.

GRUBER (W.). Monographie der bursæ mucosæ cubitales. $4^{\circ}$. St. Petersburg, 1866.

GRUBER (W.). Ueber den musculus epitrochleo-anconeus des Menschen und der Säugethiere.

$4^{\circ}$. St. Petersburg, 1866.

GRUBER (W.). Ueber die männliche Brustdrüse und über die Gynaecomaste. $\quad 4^{\circ}$. St. Petersburg, 1866.

GRUBER (W.). Ueber das spatium intra-aponeuroticum suprasternale und dessen sacci cæci retro-sternocleidomastoidei.

$4^{\circ}$. St. Petersburg, 1867.

GRUBER (W.). Ueber die Varietäten des musculus palmaris longus. $\quad 4^{\circ}$. St. Petersburg, 1868. 
GRUBER(W.). Ueber dio Halsrippen des Menschen.

$4^{\circ}$. St. Petersburg, 1869.

GRUBER (W.). Beiträge zur Anatomie des Schädelgrundes.

$4^{\circ}$. St. Petersburg, 1869.

GRUBER (W.). Ueber den musculus anconeus 'v. des Menschen, mit vergleichend-anatomischen Bemerkungen.

4. St. Petersburg, 1870.

GRUBER (W.). Ueber einen neuen secundären Tarsalknochen, calcaneus secundarius.

$4^{\circ}$. St. Petersburg, 1871.

GRUBER (W.). Ueber das erste Intermetatarsalgelenk des Menschen.

$4^{\circ}$. St. Petersburg, 1871.

GRUBER (W.). Ueber den Stirnfontanellknochen (os fonticuli frontalis) bei dem Menschen und bei den Säugethieren. $4^{\circ}$. St. Petersburg, 1873.

GRUBER (W.). Ueber die Infraorbitalkanäle bei dem Menschen und bei den Säugethieren.

$4^{\circ}$. St. Petersburg, 1874.

GRUBER (W.). Ueber die Verbindung der Schläfenbeinschuppe mit dem Stirnbeine. $4^{\circ}$. St. Petersburg, 1874.

GRUBER (W.). Monographie über die aus wahren (hyalinischen) Cartilagines praeformirten ossicula sesamoidea in den Ursprungssehnen der Köpfe des musculus gastrocnemius bei dem Menschen und bei den Säugethieren. $4^{\circ}$. St. Petersburg, 1875.

GRUBER (W.). Monographie über das corpusculum triticeum und über die accidentelle Musculatur der Ligamenta hyo-thyreoidea lateralia. $4^{\circ}$. St. Petersburg, 1876.

GRUBER (W.). Monographie über das zweigetheilte erste Keilbein der Fusswurzel (os cuneiforme 1um bipartitum tarsi) beim Menschen. $\quad 4^{\circ}$. St. Petersburg, 1877.

GRUBER (W.). Ueber den Infraorbitalrand bei Ausschliessung des maxillare superius von seiner Bildung beim Menschen.

$4^{\circ}$. St. Petersburg, 1877. 
GRUENEWALDT (M. จ.). Ueber die Versteinerungen der silurischen Kalksteine von Bogosslowsk, ein Beitrag zur Geologie des östlichen Ural. $\quad 4^{\circ}$. St. Petersburg, 1854.

GRUENEWALDT (M. г.). Notizen über die versteinerungführenden Gebirgsformationen des Ural.

$4^{\circ}$. St. Petersburg, 1857.

GRUENEWALDT (M. v.). Beiträge zur Kenntniss der sedimentären Gebirgsformationen in den Berghauptmannschaften Iekatherinburg, Slatonst und Kuschwa.

$4^{\circ}$. St. Petersburg, 1860.

GRUNAU (H.). Teber das Flimmerethel auf dem Bauchfelle des weiblichen Frosches und ueber den Eileiterbau desselben.

8. Königsberg, 1875.

GRUNDLER (C. A.). De Camelo Dromedario.

8. Tubingoe, 1817.

GRUNDLER (M.). See Bremser (J. G.).

GRUTZNER (P.). See Hermann (L.).

GUALTIER (N.). Index Testarum Conchyliorum quæ adservantur in Museo N. Gualtieri. fol. Florentce, 1742.

GUBERNATIS (А.). Zoological Mythology.

2 vols. $8^{\circ}$. London, 1872.

GUBERNATIS (A.). Die Thiere in der indogermanischen Mythologie. (Ed. M. Hartmann.)

2 vols. $8^{\circ} . \quad$ Leipzig, 1874.

GUÉNÉE (A.). Europæorum Microlepidopterorum Index methodicus.

8. Paris, 1845.

GUÉNEEE (A.). Histoire naturelle des Insectes. Lépidoptères, Noctuelites.

3 vols. $8^{\circ}$. Paris, 1852.

GUÉNÉE (A.). Histoire naturelle des Insectes. Lépidoptères, Deltoïdes et Pyralites.

8. Paris, 1854. 
GUÉNEE (A.). Histoire naturelle des Insectes. Lépidoptères, Uranides et Phalénites. 2 vols. $8^{\circ}$. Paris, 1857.

GUERIN (F. E.). See Belatger.

GUERIN-MENÉVILLE (F. E.). Iconographie du Règne Animal do G. Cuvier.

Tome I. \& 2. Planches.

Tome 3. Texte.

8․ Paris, 1829-44.

GUERIN-MENEVILLE (F. E.). Notice sur quelques Insectes nuisibles au froment, au seigle \&c.

8. Paris, 1843.

GUÉRIN-MÉNÉVILLE (F. E.). Revue et Magasin de Zoologie.

See Periodicals: Paris.

GUÉRIN-MÊNÉVILLE (F. E.) \& PERCHERON (A.). Genera des Insectes. 1 vol. So. Paris, 1835.

GUERIN-MENEVILLE (F. E.) \& PERROTET (M.). Mémoỉre sur un Insecte et un Champignon qui ravagent les Cafiers aux Antilles.

$8^{\circ}$. Paris, 1842.

GUICHENOT (A.). See Survers \& Explorations: France.

GUISAN (F. L.). De Gymnoto Electrico.

$4^{\circ}$. Tubingce, 1819.

GUITTON (E.). Nourelle classification zoologique.

8. Paris, 1854.

GÜNTHER (A.). Fische des Neckars.

8०. Stuttgart, 1853.

GÜNTHER (A.). Colubrine Snakes in the British Museum. 12. London, 1857.

GÜNTHER (A.). Medicinische Zoologie.

$8^{\circ}$. Stuttgart, 1858.

GÜNTHER (A.). Catalogue of the Batrachia Salientia in the British Museum. 8. London, 1858. 
GÜNTHER (A.). Catalogue of the Acanthopterygian Fishes in the British Museum.

Vols. I.-VIII. 80. London, 1859-70.

GÜNTHER (A.). The Reptiles of British India.

See Ray Societr.

$4^{\circ}$. London, 1864.

GÜNTHER (A.). The Fishes of Zanzibar. See Platratr (R. L.).

GÜNTHER (A.). Fische der Südsee.

See Musedur (Godefrrox).

40. Hamburg, 1873-75.

GÜNTHER (A.). The Gigantic Land-Tortoises in the British Museum.

$4^{\circ}$. London, 1877.

GÜNTHER (A.). Introduction to the Study of Fishes.

80. Edinburgh, 1880.

GÜNTHER (A.). See Biologta Centrali-Americana.

GÜNTHER (A.). Seo 'Challenger.'

GÜNTHER (A.). See 'ErebUs' ANd 'Terror.'

GÜNTHER (A.). See Periodicals : London. Zoological Record.

GURLT (E. F.). Anatomische Abbildungen der Haussäugethiere. fol. Berlin, 1843.

GURLT (E. F.). Anatomio der Hausvögel.

8․ Berlin, 1849.

GURNEY (J. H.). Catalogue of the Raptorial Birds in the Norfolk and Norwich Museum. roy. $8^{\circ}$. London, 1864.

GURNEY (J. H.). See ANdersson (C. J.).

GURNEY (J.H., jun.). Rambles of a Naturalist in Egypt. 8. London, 1876.

GUTFLEISCH (V.) \& BOSE (F. C.). Die Käfer Deutschlands.

8. Darmstadt, 1859. 
GWILT (E. T.). Lehrbuch der pathologischen Anatomie. 2 vols. $8^{\circ}$. Berlin, 1831-32.

GYLLENHAL (L.). Insecta Suecica. Classis 1.

4 vols. $8^{\circ}$. Scaris \& Lipsice, 1808-28.

H.

HAaCK (H.). See Periodicals : Berlin.

HAAN (W. de). Monographiæ Ammoniteorum et Goniatiteorum.

80. Lugduni Batavorum, 1825.

HAAN (W. de). See FAUNA JaPonica.

HAASE (E.). Schlesiens Chilopoden. $8^{\circ}$. Breslau, 1880.

HABERLANDT (F.). Der Seidenspinner des Maulbeerbaumes.

8.. Wien, 1871.

HAECKEL (E.). Die Radiolarien.

Text and Atlas, 2 vols. fol. Berlin, 1862.

HAECKEL (E.). Biologische Studien.

8. Leipzig, 1870-77.

HAECKEL (E.). Biologie der Kalkschwämme.

3 vols. $4^{\circ}$. Berlin, 1872.

HAECKEL (E.). Arabische Korallen.

$4^{\circ} . \quad$ Berlin, 1876.

HAECKEL (E.). Anthropogenie oder Entwickelungsgeschichte des Menschen.

8. Leipzig, 1877.

HAECKEL (E.). Natürliche Schöpfungsgeschichte.

$8^{\circ}$. Berlin, 1879.

HAECKEL (E.). Das System der Medusen.

4․ Jena, 1879-81. 
HAECKEL (E.). Monographie der Medusen. Zweite Hälfte. Der Organismus. Text u. Atlas. $4^{\circ}$. Jena, 1881.

HAECKEL (E.). Indische Reisebriefe.

8. Berlin, 1883.

HAECKEL (E.). See ' Challenger.'

HAGEN (H. A.). Synonymia Libellularum Europæarum. 8. . Regimontii Prussorum, 1840.

HAGEN (H. A.), Catalogue of Neuropterous Insects in the British Museum. Part I. Termitina.

12. London, 1858.

HAGEN (H. A.). Synopsis of the Neuroptera of North America.

See Suithisonian Miscell. Coll.

8०. Washington, 1861.

HAGEN (H. A.). Bibliotheca Entomologica.

2 vols. $8^{\circ}$. Leipzig, 1862-63.

HAGEN (H. A.). Monograph of the North-American Astacidoe. 4\%. Cambridge, 1870.

HAGEN (H. A.). On some Insect Deformities.

$4^{\circ}$. Cambridge, Mass., 1876.

See Musedm : Harvard College. Memoirs.

HaGEN (H. A.). See Selys-Lonchamps (E. de).

HaGEN (H. A.). See Survers \& Explorations: U.S., WASHINGTON.

HAGENBACH (J.J.). Mormolyce; novum Coleopterorum genus.

8. Norimbergce, 1825.

HAGENOW (F. v.). Die Bryozoen der Maastrichter Kreidebildung.

$4^{\circ}$. Cassel, 1851.

HAHN (C. W.) \& HERRICH-SCHAEFFER (G. A.). Die Wanzenartigen Insecten.

9 vols. $8^{\circ}$. Nürnberg, 1831-53. 
HAHN (C. W.). Ornithologischer Atlas.

8. Nürnberg, 1834-41.

HAHN (C. W.). See Reider (J. E. v.) \& HaHN (C. W.). Fauna Boica.

HALDANE (R. C.). On the Grub Pest in Ceylon.

$8^{\circ}$. Colombo, 1881.

HALDEMAN (S. S.). Freshwater Univalves of the United States (Physidoe). $\quad$ 8º Philadelphia, 1842.

HALDEMAN (S. S.). History and Transformations of Corydalus cornutus. 4. Boston, 1848.

HALDEMAN (S. S.). Elements of Latin Pronunciation. 12. . Philadelphia, 1851.

HALIDAY (A. H.). Hymenoptera Britannica Oxyura.

8. London, 1839.

HALLER (G.). Die Milben als Parasiten der Wirbellosen. $8^{\circ}$. Halle, 1880.

HALLEZ (P.). Contributions à l'histoire naturelle des Turbellaires.

$8^{\circ}$. Lille, 1879.

HALLMAN (E.). Osteologie des Schläfenbeins.

4․ Hannover, 1837.

HALLOY (J. J. d'Omalius d'). Des Races Humaines, ou Eléments d'Ethnographie.

5th edition. $8^{\circ}$. Bruxelles \& Paris, 1869.

HAMLEL (J.). Ueber Cochenille am Ararat und über Wurzelcochenille im Allgemeinen.

$4^{\circ}$. St. Petersburg, 1833.

HAMEL (J.). Ueber Dinornis und Didus.

8. St. Petersburg, 1845.

HAMEL (J.). Der Doclo.

8.. St. Petersburg, 1848.

HAMILTON (F.), formerly BUCHANAN. See BשcHaNAN (F. H.). 
HaMMER (F. L.). See Hermany (J.).

HANCOCK (A.). See ALdER (J.).

HANLEY (S.). The Conchologists' Book of Species. 2nd edition. $8^{\circ}$. London, 1842.

HANLEY (S.). Recent Bivalve Shells.

$8^{\circ}$. London, 1842-56.

HANLEY (S.). Catalogue of Recent Shells.

$8^{\circ}$. London, 1855.

HANLEY (S.). Ipsa Linnæi Conchylia.

$8^{\circ}$. London, 1855.

HANLEY (S.). See Forbes (E.).

HANLEY (S.) \& THEOBALD (IV.). Conchologia Indica. $4^{\circ}$. London, 1876.

HANNOVER (A.). La Retine de l'Homme et des Vertébrés.

4․ Paris, 1876.

HANSEN (G. A.). Seo Survers \& Explorations : Norway.

HARDWICKE (Major-General). See Grax (J. E.).

\section{HARDWICKE'S DRAWINGS.}

Mammalia. 2 rols. fol. 10,974-5.

Birds of India. 8 vols. fol. 10,976-82.

Birds, by Lewin and Withers. 1 vol. fol. 10,983.

Birds, Chinese. 1 vol. fol. 10,984.

Birds, Drawings of. 5 vols. fol. 10,986-90.

Birds, Eggs and Nests. 1 vol. fol. 10,991.

Reptiles of India. 2 vols. fol. 10,992-3.

Fishes. 4 vols. fol. 10994-7.

Crustacea. 1 vol. fol. 10,998.

Insects, Suctorial. 1 rol. fol. 10,999. 
Insects, Mandibulata. 1 vol. fol. 11,000.

Insects, Larvæ of. 1 vol. $4^{\circ} . \quad 11,001$.

Spiders. 1 vol. $4^{\circ}$. 11,002.

Insects of India. 1 vol. $4^{\circ} .11,003$.

Coleoptera. 1 vol. $4^{\circ}$. 11,004.

Mollusca and Radiata. 1 vol. fol. 11,007.

Miscellanea. 1 vol. fol. 11,008-9.

HARLAN (R.). Fauna Americana.

80. Philadelphia, 1825.

HARLAN (R.). Medical and Physical Researches.

8. Philadelphia, 1835.

HAROLD (E.. .). Coleopterologische Hefte. Parts 1-16. $8^{\circ}$. München, 1867-79.

HAROLD (E. v.). See Periodicals : Monich.

HARRIS (MI.). The Aurelian: or Natural History of English Insects. fol. London, 1778. A new edition by J. O. Westwood. fol. London, 1840.

HARRIS (M.). An Exposition of English Insects. $4^{\circ}$. London, 1782.

HARRIS (T. W.). A Treatise on some of the Insects Injurious to Vegetation. 3rd edition. $8^{\circ}$. Boston, 1862.

HARRIS (T. W.). Entomological Correspondence. Edited by S. H. ScUdDer. $\quad 8^{\circ}$. Boston, 1869. (See Periodicals: Boston, U.S.)

HARRIS (W. C.). Game and Wild Animals of Southern Africa.

fol. London, 1840. The same. 5th edition. $8^{\circ}$. London, 1852.

HARTENFELSZ (G. C. P.). Elephantographia curiosa, seu Elephanti descriptio. $4^{\circ}$. Erfordice, 1715.

HARTIG (T.). Naturgeschichte der Hymenopteren.

80. Berlin, 1860. 
HARTING (J. E.). The Birds of Middlesex : a Contribution to the Natural History of the County.

post $8^{\circ}$. London, 1866.

HARTING (J. E.). The Ornithology of Shakespeare critically examined, explained, and illustrated.

8. London, 1871.

HARTING (J. E.). Hints on Shore Shooting: with a chapter on Skinning and Preserving Birds.

post $8^{\circ}$. London, 1871.

HARTING (J. E.). A Handbook of British Birds.

8. London, 1872.

HARTING (J. E.). Rambles in search of Shells: Land and Freshwater.

8. London, 1875.

HARTING (J. E.). Our Summer Migrants : an Account of the Migratory Birds which pass the Summer in the British Islands.

8. London, 1875.

HARTING (J. E.). The Fauna of the Prybilov Islands.

8. London, 1875.

HARTING (J. E.). British Animals extinct within Historic Times. $\quad 4^{\circ}$. London, 1880.

HARTING (J. E.). Glimpses of Bird-Life pourtrayed with pen and pencil by J. E. H. and L. P. RoвERT.

4\%. London, 1880.

HARTING (J. E.). Sketches of Bird Life from twenty years' observation of their haunts and habits.

8. London, 1882.

HARTING (J. E.). Essays on Sport and Natural History.

8. London, 1883.

HARTING (J. E.) \& MOSENTHAL (J. de). Ostriches and Ostrich-farming. $\quad$ 2nd edition. $8^{\circ}$. London, 1879.

HaRTiNg (J. E.). See Periodicals : London. The Zoologist. 
HARTING (J. E.). See Rodd (E. H.).

HARTING (J. E.). See White (Gilbert).

HARTLAUB (G.). Systematisches Verzeichniss der naturhistorischen Sammlung der Gesellschaft Museum. $1^{\text {ste }}$ Abth. Vögel. 40. Bremen, 1844.

HARTLAUB (G.). Systematischer Index zu Azara's Apuntamientos para la Historia Natural del Paraguay.

4. Bremen, 1847.

HARTLAUB (G.). Beitrag zur Ornithologie Westafrica's. $4^{\circ}$. Hamburg, 1850-52.

HARTLAUB (G.). System der Ornithologie Westafrica's.

8. Bremen, 1857.

HARTLAUB (G.). Ornithologischer Beitrag zur Fauna Madagascars. 8. Bremen, 1861.

HARTLAUB (G.). Die Vögel Madagascars.

8. Halle, 1877.

HARTMANN (A.). Die Kleinschmetterlinge des Europäischen Faunengebietes.

$8^{\circ}$. München, 1880.

HARTMANN (G. L.). Helvetische Ichthyologie.

8.. Zürich, 1827.

HARTMANN (R.). Naturgeschichtlich-medicinische Skizzo der Nilländer.

8. Berlin, 1865.

HARTMANN (R.). Der Gorilla. $\quad 4^{\circ}$. Leipzig, 1880.

HARTMANN (R.). Die Menschenähnlichen Affen.

8.. Leipzig, 1883.

HARTWIG (G.). Das Leben des Meeres.

8. Frankfurt am M., 1859.

HARVEY (J. R.), HUMPHREYS (J. D.), \& POWER (J.). Contributions towards a Fauna and Flora of the County of Cork.

8. London \& Cork, 1845. 
HARVEY (W. H.). The Sea-side Book.

$12^{\circ}$. London, 1849.

HARVIE-BROWN (J. A.). The Capercaillie in Scotland. 8․ Edinburgh, 1879.

HARVIE-BROWN (J. A.). See Brown (J. A. Harvie).

HASSE (C.). De Cochlea Avium.

4. Kilice, 1866.

HASSE (C.). Die vergleichende Morphologie und Histologie des häutigen Gehörorganes der Wirbelthiere.

8. Leipzig, 1873.

HASSE (C.). Das natürliche System der Elasmobranchier. 2 parts. $4^{\circ}$. Jena, 1879-82.

HASWELL (W. A.). Catalogue of the Australian Stalkand Sessile-eyed Crustacea. $\quad 8^{\circ}$. Sydney, 1882.

See Museum (Australian).

HAWORTH (A. H.). Prodromus Lepidopterorum Britannicorum. $4^{\circ}$. London, 1802.

HAWORTH (A. H.). Lepidoptera Britannica.

8. London, 1803.

HAYDEN (F. V.). See Survers \& Explorations: U.S., WASHINGTON.

HAYES (W.). Portraits of Rare and Curious Birds from the Menagerie of Osterly Park. $4^{\circ}$. London, 1794.

HEBENSTREIT (J. C.). De organis Piscium externis. $4^{\circ}$. Lipsice, 1733.

HECKEL (J.) \& KNER (R.). Die Süsswasserfische der österreichischen Monarchie. $\quad 8^{\circ}$. Leipzig, 1858.

HECTOR (J.). Catalogue of the Land Mollusca of New Zealand. 8.. New Zealand, 1873.

HECTOR (J.). See Museda : Wellington, N. Z. 
HEDDLE (R.). See Batrie (W. B.).

HEEGER (E.). Beiträge zur Schmetterlingskunde oder Abbildungen und Beschreibungen neuer Sicilianischer Schmetterlinge.

$4^{\circ}$. Wien, 1838.

HEEGER (E.). Album microscopisch-photographischer Darstellungen aus dem Gebiete der Zoologie.

$8^{\circ}$. Wien, 1860-63.

HEER (O.). Observationes Entomologicae.

8․ Turici, 1836.

HEER (0.). Fauna Coleopterorum Helvetica.

$8^{\circ}$. Turici, 1838.

HEIDENHAIN (R.). See HeriranN (L.). Handbuch der Physiologie.

HEINCKE (F.). Die Varietäten des Herings. Zugleich ein Beitrag zur Descendenz-Theorie.

2 parts. $4^{\circ}$. Berlin, 1877-81.

HEINEMANN (H. $\nabla$.$) . Dio Schmetterlinge Deutschlands$ und der Schweiz. $\quad 3$ vols. $8^{\circ}$. Braunschweig, 1859.

HEINRICH (A.). Mährens und k.k. Schlesiens Fische, Reptilien und Vögel. 8. Brunn, 1856.

HELDREICH (T. de). La Faune de Grèce. Vertébrés. 8. Athènes, 1878.

HELLER (C.). Die Crustaceen des südlichen Europa.

8. Wien, 1863.

HELLER (C.). Die Fische Tirols und Vorarlbergs.

Innsbruck, 1871.

HELLER (C.). See ' Novara.'

HELLWIG (J. C.). See Rossi (P.).

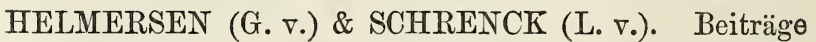
zur Kenntniss des Russischen Reiches und der angrenzenden Länder Asiens.

2 vols. 8०. St. Petersburg, 1879. 
HeMprich (F. G.). Seo Ehrenbera (C. G.).

HENDERSON (G.) \& HUME (A. O.). Lahore to Yarkand. The Expedition of 1870 under T. D. Forsyth.

$$
\text { 8.. London, } 1873 .
$$

HENLE (J.). Handbuch der systematischen Anatomie des Menschen. 3 vols. $8^{\circ}$. Braunschweig, 1855-71.

HENLE (J.) \& KÖLLIKER (A.). Ueber die pacinischen Körperchen an den Nerven des Menschen und der Säugethiere. $4^{\circ}$. Zïrich, 1844.

HENNEGUY (F.). See Batbiani (G.).

HENNIUS (H. C.). See Swamamerdam (J.).

HENSEN (V.). See Hermani (L.). Handbuch der Physiologie.

HENSEN (V.). See Meyer (H. A.) and others.

HENSHAW (H. W.). See Surverys \& Explorations: U.S., WASHINGTON.

HENTZ (N. M.). The Spiders of the United States. Edited by E. Burgess. Descriptions by J. E. Eurerton.

8. Boston, 1875.

'HERALD.' The Zoology of Voyage of H.M.S. ' Herald,' under the command of Capt. H. Kellett, 1845-51. Edited by E. Forbes. $\quad 4^{\circ}$. London, 1852.

Vertebrata. By Sir J. Richardson.

HERBELL (J. F.). See CAMPER (P.).

HERBST (J. F. W.) \& JABLONSKY (C. G.). Natursystem aller bekannten in- und ausländ. Inseckten.

8o \& obl. fol. Berlin, 1785-1806.

HERBST (J. F. W.). Versuch einer Naturgeschichte der Krabben und Krebse. $\quad 4^{\circ}$. Zurich, 1790-1804.

HeRCULAis (J. K. d'). See Kunckel d'Herculais. 
Herdian (W. A.). See 'Challenger.'

HERESBACH (C.). Libri Quarti de Re Rustica. Pars quæ agit de Piscinis et Piscium Vivariis. Cum præfatione H. Conringir. sm. $4^{\circ}$. Helmestadii, 1671.

HERING (E.). See Hermaxi (L.). Handbuch der Physiologie.

HERKLOTS (J. A.). Polypiers nageurs ou Pennatulides. $4^{\circ}$. Leycle, n. d.

HERKLOTS (J. A.). Bouwstoffen roor eene fauna van Nederland. 3 vols. Leiden, 1853-66.

HERKLOTS (J. A.). Symbolæ Carcinologicæ. Études sur la Classe des Crustacés. No. 1. 8. Leyde, 1861.

HERMAN (E.). Das Central-Nerrensystem ron Hirudo medicinatis.

$4^{\circ}$. München, 1875.

HERMANN (J.). Observationes Zoologicæ. Ed. F. L. HANMER. $4^{\circ}$. Paris, 1864.

HERMANN (L.) and others. Handbuch der Physiologie. Vols. I.-VI. 8. Leipzig, 1879-82.

HERMES (O.). See Fisheries : Berlin. Internationale Fischerei-Ausstellung, 1880.

HEROLD (M. J. D.). Entwickelungsgeschichte der Schmetterlinge.

Text \& Atlas. $4^{\circ}$. Cassel \& Marburg, 181 j.

HEROLD (M.). Disquisitiones de Animalium vertebras carentium in ovo formatione.

fol. Frankfurt am Main, 1838.

HERRE (R.). De Avium Passerinarum larynge bronchiali. 8.. Gryphioe, 1859.

HERRICH-SCHAEFFER (G. A. W.). Nomenclator entomologicus. Heft I. \& II. 8. Regensburg, 1835-40. 
HERRICH-SCHAEFFER (G. A. W.). Systematische Bearbeitung der Schmetterlinge von Europa.

6 vols. Text, 5 vols. Plates. $4^{\circ}$. Regensburg, 1845-56.

HERRICH-SCHAEFFER (G. A. W.). Sammlung neuer oder wenig bekannter ausser europäischer Schmetterlinge. $4^{\circ}$. Regensburg, 1850-58.

HERRICH-SCHAFFFER (G. A. W.). Neue Schmetterlinge aus Europa. $4^{\circ}$. Regensburg, 1856-61.

HERRICH-SCHAEFFER (G.). Seo HaHN (C. W.).

HERRMANNSEN (A. N.). Indicis generum Malacozoorum primordia. 3 vols. $8^{\circ}$. Cassellis, 1846-49, 1852.

HERTWIG (0. \& R.). Der Organismus der Medusen.

$4^{\circ}$. Jena, 1878.

HERTWIG (O. \& R.). Das Nervensystem und die Sinnesorgane der Medusen. $\quad 4^{\circ}$. Leipzig, 1878.

HERTWIG (R.). Zur Histologie der Radiolarien. 8. Leipzig, 1876.

HERTWIG ( $\left.\mathrm{R}_{\circ}\right)$. Der Organismus der Radiolarien.

$4^{\circ}$. Jena, 1879.

HERTWIG (R.). Die Actinien der 'Challenger' Expedition.

4\%. Jena, 1882.

HERTWiG (R.). See ' Challenger.'

HESS (W.). Beiträge zur Kenntniss der DecapodenKrebse Ost-Australiens. 80. Bonn, 1865.

HESSLING (T. von). Die Perlmuscheln und ihre Perlen. 8.. Leipzig, 1859.

HETTING (M. G.). Beretning om hrad der til Ferskvands Fiskeriernes Fremme er udfort i Tidsrummet.

8. Christiania, 1873. 
HEUDE (P. M.). Mémoires concernant l'Histoire Naturelle de l'Empire Chinois. $\quad 4^{\circ}$. Shanghai, 1880-82. [In progress.]

HEUDE (P. M.). Conchyliologie Fluviatile de la Province de Nanking. Parts I.-VIII., X. $4^{\circ}$. Paris, v. d. [In progress.]

HEUGLIN (Th. $\nabla$.$) . Reisen in Nord-Ost-Afrika.$

8. Gotha, 1857.

HEUGLIN (Th. $\nabla$.$) . Beiträge zur Fauna der Säugethiere$ N. O.-Afrika's. $4^{\circ}$. Jena, 1861.

HEUGLIN (Th. จ.). Beiträge zur Zoologie Africa's. 4. Dresden, 1863.

HEUGLIN (Th. $\nabla$.$) . Antilopen und Büffel N.O.-Africa's.$ $4^{\circ}$. Jena, 1863.

HEUGLIN (M. Th. v.). Reise in das Gebiet des Weissen Nil, und seiner westlichen Zuflüsse in 1862-64.

Leipzig \& Heidelberg, 1869.

HEUGLIN (Th. $\nabla$.$) . Ornithologie Nordost Africa's.$

8. Cassel, 1869-75.

HEUSINGER (C. F.). De Metamorphosi Postri Pici.

4\%. Jence, 1821.

HEUSINGER (C. F.). Vier Abbildungen des Schädels der Simia Satyrus. $4^{\circ}$. Marburg, 1838.

HEWITSON (W. C.). Coloured Illustrations of the Eggs of British Birds. 2 vols. $8^{\circ}$. London, 1846. The same. 3 rd edition. 2 vols. $8^{\circ}$. London, 1856.

HEWITSON (W. C.). Illustrations of new Species of Exotic Butterflies. $\quad 5$ vols. $4^{\circ}$. London, 1856-76.

HEWITSON (W. C.). Illustrations of Diurnal Lepidoptera. Lyconidce. 2 vols. $4^{\circ}$. London, 1862-78.

HEWITSON (W. C.). See Krrbi (W. F.). 
HEWITSON (W. C.), MOORE (F.), \& GROTE (A.). Descriptions of new Lepidopterous Insects from the Collection of the late Mr. W. S. Atkinson.

Parts I. \& II. $\quad 4^{\circ}$. Calcutta, 1879-82.

HEYDEN (L. v.), REITTER (E.), \& WEISE (J.). Catalogus Coleopterorum Europæ et Caucasi.

3rd edition. $8^{\circ} . \quad$ Berolini, 1883.

HEYDENREICH (G. H.). Lepidopterorum Europæorum Catalogus methodicus.

8. Leipzig, 1851.

HIDALGO (J. G.). Moluscos del viaje al Pacífico 1862-65. Part I. Univalvos terrestres. $\quad 4^{\circ}$. Madrid, 1869-70.

HIDALGO (J. G.). Moluscos marinos de España, Portugal, y las Baleares.

8. Madrid, 1870-74.

HIDALGO (J. G.). Catálogo Iconográfico y descriptivo de los Moluscos terrestres de España, Portugal, y las Baleares.

$8^{\circ}$. Madrid, 1875.

HIGGINS (H. H.). Synopsis of Invertebrate Animals in the Free Public Museum of Liverpool.

8. Liverpool, 1874.

HILGENDORF (F.). See Von DER Decken's Reisen.

HILL (J.). A History of Animals. fol. London, 1752.

HILL (J.). A Decade of Curious Insects.

4.. London, 1773.

HILL (P.). Natural History of Quadrupeds.

3 vols. $8^{\circ}$. Edinburgh, 1791.

HIMLY (K.). Ueber das Zusammenkugeln des Igels.

4. Braunschweig, 1801.

HINCKS (T.). A History of the British Hydroid Zoophytes. 2 vols. $8^{\circ}$. London, 1868.

HINCKS (T.). A History of the British Marine Polyzoa. 2 vols. $8^{\circ}$. London, 1880. 
HIS (W.). Cntersuchungen über die erste Allage des Wirbelthierleibes. $4^{\circ}$. Leipzig, 1868.

HIS (W.). Tntersuchungen über das Ei und die Entwickelung bei Knochenfischen.

4․ Leipzig, 1873.

HIS (W.). See Rütmreyer (L.). Crania Helretica.

HIS (W.). See Periodicals : Leipzig.

HITCHCOCK (R.). Synopsis of the Freshwater Rhizopods. 8․ New York, 1881.

HODGSON (A. E.). An Exchange List of British Coleoptera.

8. London, 1882.

HODGSON (B. H.). See Mrsecar (British).

HOEK (P. P. C.). See ' Challenger.'

HOENINGHAUS (F. W.). Beitrag zur Monographie der Gattung Crania. 4\%. Crefeld, 1828.

HOEVEN (J. van der). Dissertatio de Sceleto Piscium. 8. Lugduni Batavorum, 1822.

HOETEN (J. ran der). Icones ad illustrandas Coloris mutationes in Chamaleonte.

$4^{\circ}$. Lugduni Batavorum, 1831.

HOEVEN (J. van der). Beschrijving van Buprestis daleni, Papilio payeni, en Colias verhuellii. $\quad 8^{\circ}$. 1838.

HOEVEN (J. van der). L'Anatomie des Limules.

fol. Leyde, 1838.

HOEVEN (J. van der). Beschrijving van eenige nieuwe of wenig bekende uitlandsche soorten van Lepidoptera.

8. Leyde, 1845.

HOEVEN (J. van der). Handbuch der Zoologie.

2 vols. $8^{\circ}$. Leipzig, 1850-56. 
HOEVEN (J. van der). Hand-book of Zoology. Translated from the second Dutch edition by W. Clark.

2 vols. $8^{\circ}$. Cambridge, 1856-58.

HOEVEN (J. van der). Philosophia Zoologica.

Lugduni Batavorum, 1864.

HOEVEN (J. van der). Ontleed- en dierkundige Bijdragen tot de Kennis van Menobranchus. 4º Leiden, 1867.

HOFFER (E.). Die Hummeln Steiermarks.

2 parts in 1 vol. $8^{\circ}$. Graz, 1882-83.

HOFFMAN (C. K.). See Periodicals : Leiden.

HOFFMANN (E.). Isoporien der Europäischen Tagfalter. $8^{\circ}$. Stuttgart, 1873.

HoffManN (E.). See Pradn (S. von).

HOFFMANN (J.). Die Waldschnepfe.

$8^{\circ}$. Stuttgart, 1867.

HOFFMANN (J. J.). See Ivsektengeschichte.

HOFFMEISTER (W.). De vermibus quibusdam ad genus Lumbricorum pertinentibus. $4^{\circ}$. Berotini, 1842.

HOEFNEISTER (W.). Die bis jetzt bekannten Arten ans der Familie der Regenwürmer.

4. Braunschweig, 1845.

HÖFNAGEL (A. J.). Diversæ Insectorum Volatilium Icones. obl. $4^{\circ}$. Antwerp, 1630.

HOHNBAUM-HORNSCHUCH (R. F. M.). De Anguillarum Sexu ac Generatione. $4^{\circ}$. Gryphice, 1842 .

HOLBÖLL (C.) \& PAULSEN (J. H.). Ornithologischer Beitrag zur Fauna Grönlands. 8. Leipzig, 1846.

HOLBROOK (J. E.). North American Herpetology. 5 vols. $4^{\circ}$. Philadelphia, 1842.

I 63 ar 2


HOLBROOK (J. E.). Ichthyology of South Carolina.

$4^{\circ}$. Charleston, 1860 .

HOLDEFLEISS (T.). See Fisheries: Berlin. Internationale Fischerei-Ausstellung, 1880.

HOLDER (H. v.). Zusammenstellung der in Würtemberg vorkommienden Schädelformen. $4^{\circ}$. Stuttgart, 1876.

HOLDSWORTH(E. W. H.) \& YOUNG (A.). Sea-Fisheries and Salmon Fisheries. (In "British Industries" Series. Ed. Beavan.) 8. London, 1877.

HOLLAND (T.): Pterologische Untersuchungen.

8.. Greifswald, 1868.

HOLLARD (H.). Nouveaux Eléments de Zoologie.

8.. Paris, 1838.

HOLME (F.). On the earlier notices relating to the Natural History of the Giraffe. $\quad 8^{\circ}$. London, 1838.

HOLMGREN (A. E.). See ' Eugenie.'

HOLUB (E.) \& PELZELN (A. von). Beiträge zur Ornithologie Südafricas.

8․ Wien, 1882.

HOME (E.). Lectures on Comparative Anatomy.

6 vols. $4^{\circ}$. London, 1814-28.

HOMEYER (E. F. von). Deutschlands Säugethiere und Vögel, ihr Nutzen und Schaden. $\quad 8^{\circ}$. Leipzig, 1877.

HOMEYER (E. F. von). Die Wanderungen der Vögel mit Rücksicht auf die Züge der Säugethiere, Fische und Insecten. $\quad 8^{\circ}$. Leipzig, 1881.

HONNORAT (S. J.). Propositions sur l'histoire naturelle chimique et médicale des Cantharides. $\quad 4^{\circ}$. Paris, 1807.

HOOG (G. de). Dissertatio Medica de apologia, nexu et reciprocæ applicationis utilitate medicinæ humanæ et animalium rustico-domesticorum, sive veterinariæ artis non neganda. $\quad 4^{\circ}$. Lugduni Batavorum, 1811. 
HOPE (F. W.). A Catalogue of Hemiptera in the collection of the Rev. F. W. Hope.

8.. London, 1837.

HOPE (F. W.). The Coleopterist's Manual.

8. London, 1837-40.

HOPE (F. W.). Catalogue of the Lucanoid Coleoptera.

8. London, 1845.

HOPFFER (H. C.). See KugG (F.) \& HopfFer (H. C.).

HOPPE (D. H.). Enumeratio Insectorum elytratorum.

8. Erlanga, 1795.

HORE (H. F.). An inquiry into the Salmon and Sea Fisheries of Ireland. $\quad 8^{\circ}$. Dublin, 1850

HORN (G.). An entire new Treatise on Leeches.

8. London, 1798.

HORN (G. H.). See Lie Conte (J. L.).

HORNSCHUCH (C. F.). See Steenstrup (J. J. S.).

HORNSCHUCH (C. F.). See Sundevalu (C. J.).

HORNUNG (E. G.). Grundlage zu einem Verzeichniss der Käfer des Harzes. $\quad 8^{\circ}$. Aschersleben, 1844.

HORSFIELD (T.). Zoological Researches in Java.

$4^{\circ}$. London, 1824.

HORSFIELD (T.). Catalogue of the Mammalia in the Museum of the Hon. East India Company.

See Museum (Indian).

$8^{\circ}$. London, 1851.

HORSFIELD (T.) \& MOORE (F.). A Catalogue of the Birds in the Museum of the Hon. East India Company.

See Museum (Indian).

2 vols. 8०. London, 1854.

HORSFIELD (T.) \& MOORE (F.). Catalogue of the Lepidopterous Insects in the Museum of the Hon. East India Company.

2 vols. 80. London. 1857-59.

See Museum (Indian). 
HORVATH (G.). Monographia Lygæidaruin Hungariæ. $4^{\circ}$. Budapest, 1875.

HOUGHTON (W.). British Freshwater Fishes.

2 vols. $4^{\circ}$. London, 1879.

HOUZEA.U (J. C.). Études sur les Facultés Mentales des Animaux comparées à celles de l'Homme.

2 vols. $8^{\circ}$. Mons, 1872.

HUBER (F.). Now Observations on the Natural History of Bees. 3rd edition. $8^{\circ}$. Edinburgh, 1821.

HUBER (P.). Recherches sur les Mœurs des Fourmis Indigènes.

8. Paris, 1810.

HUBER (P.). The Natural History of Ants. Translated from the French by J. R. JoHnson. $\quad 8^{\circ}$. London, 1820.

HÜBNER (F. L.). De organis motoriis Boæ caninæ.

4․ Berolini, 1815.

HÜBNER (J.). Beiträge zur Geschichte der Schmetterlinge. 8․ Augsburg, 1786-89.

HÜBNER (J.). Sammlung Europäischer Schmetterlinge. 5 vols. $4^{\circ}$. Augsburg, 1805-41.

HÜBNER (J.). Verzeichniss bekannter Schmetterlinge. 8. Augsburg, 1816.

HÜBNER (J.). Systematisch-alphabetisches Verzeichniss aller zur Sammlung Europäischer Schmetterlinge angegebenen Gattungsbenennungen. $8^{\circ}$. Augsburg, 1822.

HUBRECHT (A. A. W.). Aanteekeningen over de Anatomie Histologie en ontwikkelingsgeschiedenis van eenige Nemertinen.

8. Utrecht, 1874.

HUBRECHT (A, A. W。). See Bronn : Thierreich.

HUDSON and SON. Treatise on the Medicinal Leech.

$8^{\circ}$. Hull, 1841. 
HUEBNER (C. W. F.). Wallthier: Balona longimana. 4. Reval, 1852.

HUET (J. B.). Collection des Mammifères du Museum d'histoire naturelle à Paris. $4^{\circ}$. Paris, 1808.

HUGUES (P.). Storia Naturale delle Scimie e dei Maki. fol. Milano, 1817.

HUGUES (P.). Natur-Geschichte der Affen und der Maki. fol. Milano, 1823.

HUITRES. Dissertation sur les Huitres vertes do Marennes. Par G.... de la B.... 8 8. Rochefort, 1821.

HUMibERT (A.). See Explorations \& Surveys : France.

HUMBOLDT (A.) \& BONPLAND (A.). Beobachtungen aus der Zoologie und Vergleichenden Anatomie, Gesammelt auf einer Reise nach den Tropen Ländern in den Jahrem 1799-1804. $\quad 4^{\circ}$. Tübingen \& Paris, 1806.

HUMBOLDT (A.) \& BONPLAND (A.). Voyage de H. et B. Deuxième partie. Observations de Zoologie et d'Anatomie comparée. $\quad 2$ vols. in 1. $4^{\circ}$. Paris, 1811.

HUME (A.). Stray Feathers.

See Periodicals: Calcutta.

HUME (A.). Nests and Eggs of Indian Birds.

3 parts. $8^{\circ}$. Calcutta, 1873-75.

HUME (A.) \& MARSHALL (C. H. T.). The Game Birds of India, Burmah, and Ceylon. 3 vols. $8^{\circ}$. Simla, 1879.

HUME (A. O.). See Henderson (G.) \& HuME (A. O.).

HUMMEL (A. D.). Essais Entomologiques. sm. $8^{\circ}$. St. Petersburg. 1821.

HUMMEL (A. D.). Supplementum ad Faunæ Ingricæ Prodromum. Eleutherata. $4^{\circ}$. Moscow, 1823. 
HUMPHREYS (H. N.). The Genera of British Yoths. roy. $8^{\circ}$. London, 1858-59.

HUMPHREYS (H. N.) \& WESTWOOD (J. O.). British Butterflies and their Transformations.

1 vol. $4^{\circ}$. London, 1841.

HUMPHREYS (H. N.) \& WESTWOOD (J. O.). British Moths and their Transformations.

2 vols. $4^{\circ}$. London, 1851.

HUMPHREYS (J. D.). See HaRvey (J. R.).

HUMPHRY (G. M.). On the Limbs of Vertebrate Animals. 4\%. Cambridge, 1860.

HUNICH (L. A.). Bestimmung der esperschen Abbildungen Europäischer Schmetterlinge. $\quad 4^{\circ}$. Leipzig, 1854.

HUNTER (J.). Essays on Natural History, Anatomy, Physiology, Psychology, and Geology. Edited by R. Owen. 2 vols. $8^{\circ}$. London, 1861.

HUNTER (J.). See Musedy: College of Surgeons.

HUNTER (W. P.). Selections from the Natural History of the Quadrupeds of Paraguay and the River La Plata.

8. London, 1837.

HUPPERT (H.). See Hermand (L.). Handbuch der Zoologie.

HURDIS (J. L.). See Jones (J. M.).

HUSCHKE (A.). Commentatio de Pectinis in Oculo Avium. $4^{\circ}$. Jence, 1827.

HUTH (G. L.). See Parsons (J.).

HUTTON (F. W.). The Birds of New Zealand.

8. New Zealand, 1871.

HUTTON (F. W.). The Fishes of New Zealand.

80. New Zealand, 1872. 
HUTTON (F. W.). Echinodermata of New Zealand.

8. New Zealand, 1872.

HUTTON (F. W.). Marine Mollusca of New Zealand.

$8^{\circ}$. Wellington, 1873 and 1880.

HUTTON (F. W.). Tertiary Mollusca and Echinodermata of New Zealand.

$8^{\circ}$. Wellington, 1873.

HUTTON (F. W.). Manual of the New Zealand Mollusca. $8^{\circ}$. Wellington, 1880.

HUTTON (J.). See Daumas (E.).

HUXLEY (T. H.). Oceanic Hydrozoc. fol. London, 1859. See Ray SocIETy.

HUXLEY (T. H.). Man's Place in Nature.

8. London \& Edinburyh, 1863.

HUXLEY (T. H.). Lectures on the Elements of Comparative Anatomy, on the Classification of Animals, and on the Vertebrate Skull.

8. London, 1864.

HUXLEY (T. H.) \& HAWKINS (B. W.). Comparative Osteology, in twelve plates. 4\%. London, 1864.

HUXLEY (T. H.). Introduction to the Classification of Animals.

$8^{\circ}$. London, 1869.

HUXLEY (T. H.). Anatomy of Vertebrated Animals.

$8^{\circ}$. London, 1871.

HUXLEY (T. H.). A Manual of the Anatomy of Invertebrated Animals. $\quad 12^{\circ}$. London, 1877.

HUXLEY (T. H.). The Crayfish.

3rd edition. $8^{\circ}$. London, 1881.

HUXLEY (T. H.). See LAING (S.).

HYRTL (J.). Cryptobranchus Japonicus.

$4^{\circ} . \quad$ Vindobonce, 1865.

HYRTL (J.). Das Arabische und Hebräische in der Anatomie.

8०. Wien, 1879. 
IBIS (The). See Periodicals : London.

IHERING (H. von). Die Gehörwerkzeuge der Mollusken.

8. Erlangen, 1876.

IHERING (H. von). Vergleichende Anatomie des Nervensystemes und Phylagenie der Mollusken.

$4^{\circ}$. Leipzig, 1877.

IHERING (H. von). Das peripherische Nervensystem der Wirbelthiere.

$4^{\circ}$. Leipzig, 1878.

ILLIGER (J. C. W.). Verzeichniss der Käfer Preussens. 8. Halle, 1798.

ILLIGER (J. C. W.). Terminologie für das Thierreich und Pflanzenreich.

$8^{\circ}$. Helmstädt, 1800.

ILLIGER (J. C. W.). Olivier's Entomologie. $4^{\circ}$. Braunschweig, 1800.

ILLIGER (J. C. W.). Magazin für Insektenkunde. 6 vols. in 4. $8^{\circ}$. Braunschweig, 1802-7.

ILLIGER (J. C. W.). Prodromus systematis Mammalium et Avium.

8.. Berolini, 1811.

ILLIGER (J. C. W.). Tabellarische Uebersicht der Vertheilung der Vögel. $4^{\circ}$. Berlin, 1812.

ILLIGER (J. C. W.). Ueberblick der Säugethiere.

4\%. Berlin, 1815.

ILLIGER (J. C. W.). See Rossi (P.).

IMHOFF (L.). Versuch einer Einführung in das Studium der Koleoptern.

$8^{\circ}$. Basel, 1856.

IMHOFF (L.). See Labram (D.).

IM THURM (E. F.). The Birds of Marlborough.

8. Marlborough, 1870. 
INGERSOLL (E.). See Fisheries: Washington.

INGPEN (A.). Instructions for collecting Insects.

2nd edition. $12^{\circ}$. London, 1839.

INSECTA Britannica. Lepidoptera by H. T. Stannton.

Diptera by F. WALKER.

8. London, 1854.

3 vols. $\quad 8^{\circ} . \quad$ London, 1851-56.

INSEKTENGESCHICHTE. Ausgearbeitet von einigen Freunden der Naturgeschichte.

2 vols. in 1. $8^{\circ}$. Frankfurt, 1803.

IRBY (H. L.). Ornithology of the Straits of Gibraltar.

8. London, 1875.

ISER (C.). S Svensk Entomologi. $\quad 8^{\circ}$. Linköping, 1806.

ISSEL (A.). Malacologia del Mar Rosso.

8.. Pisa, 1869.

IVENS (W.). De Rege Rattorum et Felium.

8. Kilice, 1842.

IZJONVAL (D. B. d'). See CAMPER (P.).

J.

JACOB IEUS (0.). De Ranis et Lacertis Observationes.

$12^{\circ}$. Hafnio, 1686.

JACOBADS (0.). Museum Regium seu Catalogus Rerum tam naturalium quam artificialium quæ in Basilica Bibliothecæ Friderici IV. Havniæ asservantur.

fol. Havnice, 1696.

JACOBI (H. S. R.). De vesica aërea Piscium.

$4^{\circ}$. Berolini, 1840.

JACOBS (F. G. J.). Talpæ Europeæ Anatome.

$8^{\circ}$. Jena, 1816. 
JACOBY (L.). Der Fischfang in der Lagune von Commacchio nebst einer Darstellung der Aalfrage.

$8^{\circ}$. Berlin, 1880.

JaCOBY (M.). See Biologia Cextrali-Americana.

JACQUELIN DU VAL (C.). Glanures Entomologiques. 16. Paris, 1859-60.

JACQUELIN DU VAL (C.). Manuel Entomologique. Genera des Coléoptères d'Europe.

4 vols. $12^{\circ}$. Paris, $1857-68$.

JACQUEMONT (V.), Correspondance de, pendant son royage dans l'Inde. $\quad 3$ vols. $\quad 8^{\circ}$. Paris, 1833.

JACQUIN (J. F. E.). Beyträge zur Geschichte der Vögel. 4. Wien, 1784.

JAFFE (L. M.). De Ornithorhyncho paradoxo.

$4^{\circ}$. Berolini, 1823.

J ̈̈GER (A.). Beobachtungen über die Anatomie des Nilcrocodils.

8. . Tübingen, 1837.

J̈̈GER (G.). Das Os Humeroscapulare der Vögel.

8. Tübingen, 1858.

J̈̈GER (G.). Handwörterbuch der Zoologie, Anthropologie und Ethnologie. 2 vols. roy. $8^{\circ}$. Breslau, 1880-83.

JäGER (G. F.). De Holothuriis. $\quad$ 4º Turici, 1833.

J̈̈GER (H. F.). Anatomische Untersuchungen des Orycteropus capensis. $\quad 4^{\circ}$. Stuttgart, 1837.

JAN (G.). Cenni sul Museo Cirico di Milano, ed Indice sistematico dei Rettili ed Anfibi. $\quad 8^{\circ}$. Milano, 1837.

JAN (G.). Elenco sistematico degli Ofidi.

8. Milano, 1863.

JAN (G.). Iconographie générale des Ophidiens.

Texte, $8^{\circ}$. Atlas, $4^{\circ}$. Paris, 1860-78. 
JAN (J. C. \& G.). Catalogus rerum Naturalium in Museo exstantium.

8. Milano, 1832.

JANIN (J.). See BoItaRd (M. P.).

JANSE (G.). Catalogus bibliotheek van het Koninklyk Zoologisch Genootschap "Natura Artis Magistra" to Amsterdam. roy. $8^{\circ}$. Amsterdam, 1881.

Janson (E. W.). See Cistula ; and Biologia ChantraliAMERICANA.

JARDINE (W.), The Naturalist's Library.

40 vols. $88^{\circ}$. London, 1845.

JARDINE (W.). British Salmonidæ.

Atlas fol. Edinburgh, 1839-41.

JARDINE (W.). Contributions to Ornithology, 18481852. 2 vols. $8^{\circ}$. London, 1867.

JARDINE (W.) \& SELBY (P. J.). Illustrations of Ornithology. 4 vols. $4^{\circ}$. Edinburgh, 1825-43.

JARDiNE (W.). See Periodicals: London. Magazine of Zoology and Botany.

JARDINE (Sir W.). See Strickland (H. E.).

JAROCKI (F. P. von). Zubr, oder der Lithauische Auerochs.

$8^{\circ}$. Hamburg, 1830.

JASSOY (E. T.). De Echinorhyncho polymorpho Bremseri.

4\%. Herbipoli, 1820.

JAUBERT (J. B.) \& BARTHÉLEMY-LAPOMMERAYE. Richesses Ornithologiques du midi de la France.

$4^{\circ}$. Marseille, 1859.

JAY (J. C.). A Catalogue of Recent Shells.

2nd edition. $8^{\circ}$. New York, 1836.

4th edition. $8^{\circ}$. New York, 1850.

JAYNE (H. F.). Revision of the Dermestidae of the United States. 8. Philadelphia, 1882. 
JEFFREYS (J. Gwyn). British Conchology.

5 vols. $8^{\circ}$. London, 1862-69.

JEITTELES (L. H.). Ueber einige seltene und wenig bekannte Säugethiere des südöstlichen Deutschlands.

8.. St. Pölten, 1867.

JEKEL (H.). Genera et species Curculionidum.

See ScHönheRr (C. J.).

12․ Paris, 1849 .

JEKEL (H.). Insecta Saundersiana.

Part 1. 8․ London, 1855.

JENNINGS (J.). Ornithologia, or the Birds.

post $8^{\circ}$. London, 1828.

JENSEN (O. S.). Turbellaria ad litora Norvegiæ occidentalia.

$4^{\circ}$. Bergen, 1878.

JENTINK (F. A.). See Musedm : Leyden.

JENYNS (L.). A Manual of British Vertebrate Animals. $8^{\circ}$. Cambridge, 1835.

JENYNS (L.). See 'Beagle.'

JENYNS (L.). Observations in Natural History.

$8^{\circ}$. London, 1846.

JERDON (T. C.). Illustrations of Indian Ornithology.

$4^{\text {o. }}$ Madras, 1847.

JERDON (T. C.). The Birds of India.

3 vols. $8^{\circ} . \quad$ Calcutta, 1862-64.

JERDON (T. C.). The Mammals of India.

8. Calcutta, 1867.

JERDON (T. C.). Notes on Jerdon's Mammals of India. See MacMaster.

8. Madras, 1870.

JERMYN (L.). The Butterfly Collector's Vade Mecum. 3rd edition. $8^{\circ}$. London, 1836. 
JESSE (E.). Gleanings in Natural History.

3 vols. $8^{\circ}$. London, 1832-35.

JESSE (G. R.). Researches into the History of the British Dog.

2 vols. $8^{\circ}$. London, 1866.

JETZE (T. C.). Physikoteleologische Betrachtung ïber die weissen Hasen in Liefland. $12^{\circ}$. Lubeck, 1749.

JOANNIS (L. de). Observations sur les Poissons du Nil et description de plusieurs espèces nouvelles.

$8^{\circ}$. Paris, 1835.

JOBERT DE LAMBALLE (A. J.). Des Appareils électriques des Poissons électriques.

Text, $8^{\circ}$. Atlas, fol. Paris, 1858.

JUHANNEAU (E.). See LathaM (J.).

JOHNSON (T.). Illustrations of British Hawk-Moths and their Larvæ. 8. London, 1874-76.

JOHNSON (T.). Illustrations of Exotic Conchology.

$8^{\circ}$. London, 1881.

JOHNSSON (A.). Synoptisk Framstallning af Sveriges Oniscider.

8.. Upsala, 1858.

JOHNSTON (A. K.). The Royal Atlas of Modern Geography. fol. Edinburgh \& London, 1863.

JOHNSTON (A. K.). Index Geographicus.

8.. Edinburgh \& London, 1864.

JOHNSTON (A. K.). The Physical Atlas of Natural Phenomena.

fol. Edinburgh \& London, 1856.

JOHNSTON (G.). A History of British Sponges and Lithophytes. 8. Edinburgh, 1842.

JOHNSTON (G.). A History of British Zoophytes. 8. Edinburgh, 1838. 2nd edition. 2 vols. $8^{\circ}$. London, 1847. 
JOHNSTON (G.). Introduction to Conchology.

$8^{\circ}$. London, 1850.

JOHNSTON (G.). British Non-Parasitical Worms in the British Museum.

8. London, 1865.

JOHNSTON (G.). See Periodicals: London. Magazine of Zoology and Botany.

JOHNSTON (H. H.). Report on the Natural History of Mossâmedes and District.

8.. London, 1882.

JOLY(M.). Coup d'œil sur les origines de la pisciculture fluviale et sur l'état actuel de cette industrie en France.

$8^{\circ}$. Toulouse, 1866.

JONES (H. B.). On Animal Electricity.

$8^{\circ}$. London, 1852.

JONES (J. M.). The Naturalist in Bermuda. By J.M. J., assisted by J. W. WedDERBURN and J. L. Hurdis.

8.. London, 1859.

JONES (T. Rupert). Article on Tunicata. See CaRPenter (P. P.). Lectures on Mollusca.

JONES (T. R.). The Aquarian Naturalist.

8. London, 1858.

JONES (T. R.). The Animal Kingdom.

3rd edition. $8^{\circ}$. London, 1861.

JONES (T. R.). CASSELL's Book of Birds, from the text of Dr. BreHu. 4 vols. in $2.4^{\circ}$. London, n. d.

JONSTON (J.). Historia Naturalis de Quadrupedibus.

$4^{\circ}$. Frankfurt, 1649. Another edition. $4^{\circ}$. Frankfurt, 1650-53. Another edition. fol. Amsterdam, 1657.

JORDAN (D. S.). Manual of the Vertebrates of the Northern United States. $8^{\circ}$. Chicago, 1878.

JORDaN (D. S.). See Periodicals: Washington. 
JOVIUS (P.). De Piscibus Romanis libellum.

$12^{\circ}$. Basilea, 1561.

JUNG (C. C.). Alphabetisches Verzeichniss der bisher bekannten Schmetterlinge.

12. Markbreit, 1791.

JURINE (L.). Nouvelle Méthode de classer les Hyménoptères et les Diptères.

4. Genève, 1807.

JURINE (L.). Histoire des Monocles qui se trourent aux environs de Genève.

4. Geneva, 1820.

JURINE (L.). Figures pour l'Histoire abrégée des poissons du Lac Léman. obl. 4. Genève \& Paris, 1826.

K.

KALTENBACH (J. H.). Monographie der Familien der Pflanzenläuse (Phytophthires). $\quad 8^{\circ}$. Aachen, 1843.

KAITENBACH (J. H.). Die Pflanzen-Feinde aus der Classe der Insecten. 8. Stuttgart, 1872-74.

KAROLY (L.). Über den Bau des Gastrodiscus polymastos, Leuckart. 4․ Frankfurt, 1881.

KARSTEN (D. L. G.). Museum Leskeanum.

$$
2 \text { vols. } 8^{\circ} \text {. Lipsiae, } 1789 .
$$

KARSTEN (G.). See Meyer (H. A.) and others.

KAT(H.D.). Dayboek eener Reize ter Walvisch en Robben Vangst 1777-78. 8. Haarlem, 1818.

KATTER (F.). Monographie der Europäischen Arten der Gattung Meloë. 8. Putbus, 1883.

KAULLA (H.). Monographia Hyracis.

$4^{\circ}$. I'ubingo, 1830. 
KAUP (J. J.). Classification der Säugethiere und der Vögel. 8․ Darmstadt, 1844.

KAUP (J. J.). Catalogue of Apodal Fish in the British Museum. 8. London, 1856.

KAUP (J. J.). Catalogue of Lophobranchiate Fish in the British Museum. 16. London, 1856.

KAUP (J. J.). See KLIPSTEIN (A. จ.).

KAYSER (J. C.). Deutschlands Schmetterlinge.

8․ Leipzig, [1853-59 ?].

KEFERSTEIN (A.). Entomologische Notizen aus dem Tagebuch des zu Madagascar verstorbenen Herrn Toums.

8.. n. d. [1863?].

KEFERSTEIN (A.). Betrachtungen über die Entwickelungsgeschichte der Schmetterlinge und deren Variation. 8. Erfurt, 1880.

KEFERSTEIN (W.) \& EHLERS (E.). Zoologische Beiträge. 4.. Leipzig, 1861.

KELAART (E, F.). Prodromus Faunæ Zeylanicæ.

8. Ceylon, 1852.

KELCH (A.). Grundlage zur Kenntniss der Käfer Oberschlesiens. 4․ Regensburg, 1846.

KELCH (A.). Grundlage zur Kenntniss der Orthopteren Oberschlesiens.

$4^{\circ}$. Regensburg, 1852.

KELLER (A. de). Elenchus Librorum de Apium Cultura. Bibliografia di Apicoltura.

12. Milano, 1881.

KELLER (C.). Untersuchungen über die Anatomie und Entwickelungsgeschichto einiger Spongien des Mittelmeeres. $4^{\circ}$. Basel, Genf, \& Lyon, 1876.

KELLETT (H.). See ' Herald.'

KELLNER (A.). Verzeichniss der Käfer Thüringens.

8. Erfurt, 1873. 
KENNEDY (A. W. M. C.). The Birds of Berkshire and Buckinghamshire. 8. . Eton \& London, 1868.

KENT (W. S.). A Manual of the Infusoria.

2 vols. $8^{\circ}$. London, 1880-83.

KERMODE (P. M. C.). See Brown (J. A. Harvie) and others.

KERR (R.). The Animal Kingdom. Mammalia and Birds. 4.. Londlon, 1792.

KERSTEN (H.). Capitis Trichechi rosmari Descriptio Osteologica. 8. Berolini, 1821.

KERSTEN (O.). See Von der Decken's Reisen.

KESSLER (H. F.). Lebensgeschichto von Centhorhynchus sulcicollis, Gyll., und Nematus ventricosus, Klug. Beitrag zur Kenntniss und Vertilgung schädlicher Garteninsecten.

8. Cassel, 1866.

KESSLER (H. F.). Die Lebensgeschichte der auf Ulmus campestris, L., vorkommenden Aphiden Arten.

$8^{\circ}$. Cassel, 1878.

KESSLER (H. F.). Neue Beobachtungen und Entdeckungen an den auf Ulmus campestris, L., vorkommenden Aphiden-Arten.

8. Cassel, 1880.

KESSLER (H. F.). Die auf Populus niger, L., und Populus dilatata, Ait., vorkommenden Aphiden-Arten.

$8^{\circ}$. Cassel, 1882.

KESSLER (K.). Auszïge aus dem Berichto über eine an die Küsten des Schwarzen Meeres und die Krym unternommene Reise (Fische). $\quad 8^{\circ}$. Moskau, 1859.

KESSLER (K.). Fishes of the Black and Caspian Seas compared and described.

8. St. Petersburg, 1874.

[In Russian.]

KESSLER (K.). Cray-Fish of the Genus Astacus.

[In Russian.] $\quad 8^{\circ}$. St. Petersburg, 1875. 
KESSLER (K.). Fishes which occur in the waters of the Lake Aral, and of the Caspian and Black Seas.

[In Russian.]

8. St. Petersburg, 1877.

KESSLER (L.). Zur Entwickelung des Auges der Wirbelthiere. 4‥ Leipzig, 1877.

KEULEMANS (J. G.). Onze Vogels in huis en tuin. 3 vols. $4^{\circ}$. Leyden, 1869-76.

KEYSERLING (A. G.) \& BLASIUS (J. H.). Die Wirbelthiere Europa's. 8. Braunschweig, 1840.

KEYSERLING (A. G.) \& BLASIUS (J. H.). Beschreibung einer neuen Feldmaus, Arvicola ratticeps.

$4^{\circ}$. St. Petersburg, 1841.

KEYSERLING (G.E.). Die Spinnen Amerika’s. Laterigradoe.

$4^{\circ}$. Nürnberg, 1880.

KIENER (L. C.). Species général des Coquilles vivantes. 10 vols. $8^{\circ}$. Paris, 1846-56.

KILBOURNE (S. A.) \& GOODE (G. B.). Game Fishes of the United States. obl. fol. New York, 1878-80.

KINBERG (J. G. H.). De Tragulo javanico. Dissertatio zootomica, præside Dr. N. H. Loven. $8^{\circ}$. Lundw, 1849.

KING (W. R.). The Sportsman and Naturalist in Canada. 8. London, 1866.

KIPRIJANOFF (W.). Studien über die fossilen Reptilien Russlands. $4^{0}$. St.-Pétersbourg, 1881.

KIRBY (W.). Monographia Apum Angliæ.

2 vols. $8^{\circ}$, Ipswich, 1802.

KIRBY (W.). On the Power of God as manifested in the Creation of Animals.

2 vols. $8^{\circ}$. London, 1835.

KIRBY (W.) \& SPENCE (W.). An Introduction to Entomology. 4th edition. 4 vols. $8^{\circ}$. London, 1822-26. 
KIRBY (W. F.). A Manual of European Butterflies. $12^{\circ}$. London, 1862.

KIRBY (W. F.). Catalogue of Diurnal Lepidoptera. 1 vol. in $2.8^{\circ}$. London, 1871. Supplement. $8^{\circ}$. London, 1877.

KIRBY (W. F.). Catalogue of Diurnal Lepidoptera formed by the late William Chapman Hewitson, and bequeathed by him to the British Museum.

4\%. London, 1879.

KIRBY (W. F.). European Butterflies and Moths.

$4^{\circ}$. London, 1882.

KIRCHENPAUER (G. H.). Ueber die Hydroidenfamilie Plumularidce. 4. Hamburg, 1872.

KIRCHSBERG (O.). Catalogus Coleopterorum Transilvaniæ. 8. Claudiopoli, 1870.

KITTLITZ (F. H. von). Ueber einige Vögel ron Chili. $4^{\circ}$. St. Petersburg, 1830.

KITTLITZ (F. H. von), Naturgeschichte der Vögel. 8. Frankfurt am Main, 1832.

KJ ÄRBÖLLING (N.). Ornithologia Danica. Text, 8०. Atlas, fol. Kjöbenhavn, 1851-52.

KJäRBÖLLING (N.). Skandinaviens Fugle. Ed. J. CoLLIN. $8^{\circ} \&$ fol. Kjöbenhavn, 1875-77.

KLAATSCH (H. M. A.). Do Cerebris Piscium Ostracanthorum aquas nostras incolentium.

4․ Hatis Saxonum, 1850.

KLEIN (E.), BURDON-SANDERSON (J.), FOSTER (M.), \& BRUNTON (T. L.). Handbook for the Physiological Laboratory. Text \& Atlas. 8 $8^{\circ}$. London, 1873.

KLEIN (E.) \& SMITH (E. N.). Atlas of Histology.

$4^{\circ}$. London, 1880. 
KLEIN (J. T.). Descriptiones Tubulorum Marinorum.

$4^{\circ}$. Gedani, 1731.

Another edition. $4^{\circ}$. Gedani \& Lipsice, 1773.

KLEIN (J. T.). Summa dubiorum circa classes Quadrupedum et Amphibiorum.

$4^{\circ}$. Leipzig, 1743.

KLEIN (J. T.). Historiæ Avium Prodromus.

$4^{\circ}$. Lubecce, 1750.

KLEIN (J. T.). Quadrupedum Dispositio brevisque Historia Naturalis.

4․ Leipzig, 1751.

KLEIN (J. T.). Tentamen Methodi Ostracologicæ.

$4^{\circ}$. Lugduni Batavorum, 1753.

KLEIN (J. T.). Tentamen Herpetologiæ.

$4^{\circ}$. Leiden, 1755.

KLEIN (J. T.). Stemmata Avium. $\quad 4^{\text {o }}$. Lipsice, 1759.

KLEIN (J. T.). Verbesserte und vollständigere Historie der Vögel. Ed. REGGER.

$4^{\circ}$. Danzig, 1760.

KLEIN (J. T.). Natürliche Ordnung und vermehrte Historie der vierfüssigen Thiere. Ed. REGGER.

$4^{\circ}$. Danzig, 1760.

KLEIN (J. T.). Ova Avium. [Title also in German.]

$4^{\circ}$. Leipzig, 1766.

KLEIN (J. T.). Ichthyologia Enodata, sive Index rerum ad historiam Piscium naturalem ab J. J. WaLBaUM.

$4^{\circ}$. Lepsiae, 1793.

KLEUDGEN (P.). Die deutsche Colonie Santa Cruz.

12. Hamburg, 1852.

KLIPSTEIN (A. v.) \& KAUP (J. J.). Description d'un crâne colossal de Dinotherium giganteum. $4^{\circ}$. Paris, 1837.

KLOETZKE (C. G.)。 De Rana cornuta.

$4^{\circ}$. Berolini, 1816. 
KLUG (F.). Monographia Siricum Germanı.

$4^{\circ}$. Berolini, 1803.

KLUG (F.). Entomologische Monographien.

8०. Berlin, 1824.

KLUG (F.). Jahrbücher der Insectenkunde.

8. Berlin, 1834.

KLUG (F.) \& EHRENBERG (C. G.). Symbolæ Physicæ seu icones et descriptiones Insectorum. fol. Berolini, 1829.

KLUG (F.) \& HOPFFER (C. H.). Neue Schmetterlinge der Insekten Sammlung des königl. Zool. Mus. zu Berlin.

$4^{\circ}$. Berlin, 1836-1856.

KLUNZINGER (C. B.). Synopsis der Fische des Rothen Meeres.

$8^{\circ}$. Wien, 1870-71.

KLUNZINGER (C. B.). Die Korallenthiere des Rothen Meeres. Theil I.-III. 4º Berlin, 1877.

KNAUER (F. K.). Amphibien und Reptilien-Zucht.

$8^{\circ}$. Wien, 1875.

KNAUER (F. K.). Naturgeschichte der Lurche.

$8^{\circ}$. Wien, 1878.

KNAUER (W.). See Fisheries : BerLiN. Internationalo Fischerei-Ausstellung, 1880.

KNER (D. R.). Die Hypostomiden. $\quad 4^{\circ}$. Wien, 1854.

KNER (R.). See 'Novara.'

KNOCH (A. W.). Beiträge zur Insektengeschichte. 3 vols. in 1. 80. Leipzig, 1781-83.

KNOCH (A. W.). Neue Beyträge zur Insectenkunde. 8. Leipzig, 1801.

KNOCH (J.). Die Naturgeschichte des breiten Bandwurms (Bothriocephalus latus auctt.).

$4^{\circ}$. St. Petersburg, 1862. 
KNORR (G. W.). Sammlung von Muschein.

6 vols. $4^{\circ}$. Nürnberg, 1757-72.

KNORR (G. W.), MÜLLER (P. L. S.), \& WALCH (J. E. J.). Delicice selectoe, oder auseriesenes Naturalien Cabinet. fol. Nürnberg, 1778.

KNOX (A. E.). Game Birds and Wild Fowl.

S. London, 1850.

KNOX (A. E.). Ornithological Rambles in Sussex. 3rd edition. $12^{\circ}$. London, 1855.

KNOX (Dr.). Fish and Fishing in the lone Glens of Scotland. 8.. London, 1854.

KOBELT (W.). Illustrirtes Conchylienbuch.

$4^{\circ}$. Nürnberg, 1878.

KOBELT (W.). Catalog der im Europäischen Faunengebiet lebenden Binnenconchylien.

2nd edition. $8^{\circ}$. Cassel, 1881.

KOBELT (W.). Iconographie der Schalentragenden Europäischen Meeresconchylien. Heft 1. 4 . Cassel, 1883.

KOBELT (W.). See Rossyässler (E. A.).

Kobelt (W.). See Periodicals : Frankfurt a. Main.

KOCH (C. L.). Die Pflanzenläuse, Aphiden.

8․ Nürnberg, 1854.

KOCH (C. L.). Die Myriapoden. 2 vols. $8^{\circ}$. Halle, 1863.

KOCH (F.). Die Schlangen Württembergs für landwirthschaftliche Fortbildungs- und Abendschulen, Realanstalten, lateinische und Volkschulen. $\quad 4^{\circ}$. Stuttgart, 1862

KOCH (G.). Verureitung der Europäischen Schmetterlinge. 8.. Leipzig, 1857. 
KOCH (G.). Die Indo-australische Lepidopteren-Fauna.

8. Leipzig, 1865.

2nd edition. $8^{\circ}$. Berlin, 1873.

KOCH (G.). Synopsis der Vögel Deutschlands.

12.. Heidelberg, 1871.

KOCH (G.). Die Schmetterlinge des südwestlichen Deutschlands. $\quad 8^{\circ}$. Cassel, 1856, 1880, 1881.

KOCH (G. von). Anatomie der Orgelkoralle (Tubipora Hemprichii, Ehrbg.). 8.. Jena, 1874.

KOCH (J. D. W.). See Insektengeschichte.

KOCH (K. L.). System der Baierischen Zoologie.

12․ Nürnberg, 1816.

KOCH (L.). Die Arachniden-Familie der Drassiden.

8․ Nürnberg, 1866-67.

KOCH (L.). Die Myriapodengattung Lithobius.

8.. Nürnberg, 1862.

KOCH (L.). Uebersichtliche Darstellung der Europäischen Chernetiden (Pseudoscorpione). $\quad$ 8. Nürnberg, 1873.

KOCH (L.). Die Arachniden Australiens.

[In progress.]

Parts I.-XXX. 4\%. Nürnberg, 1871-82.

KOCH (L.). Agyptische und Abyssinische Arachniden.

$4^{\circ}$. Nürnberg, 1875 .

KÖHLER (H. J. de). Aristoteles de Molluscis Cephalopodibus Commentatio.

$12^{\circ}$. Regoe, 1820.

KOLENATI (F. A.). Meletemata Entomologica. Insecta Caucasi. Fasc. I.-IV. $\quad 8^{\circ}$. Petropoli, 1845-46.

KOLLAR (V.). Monographia Chlamydum.

fol. Vienna, 1824. 
KOLLAR (V.). A Treatise on Insects injurious to Gardeners. Translated by J. and M. Loddon. With Notes by J. O. Westwood.

8. London, 1840.

KÖLLIKER (A.). Observationes de prima Insectorum genesi. $4^{\circ}$. Turici, 1842.

KÖLLIKER (A.). Die Schwimmpolypen oder Siphonophoren von Messina.

fol. Leipzig, 1853.

KÖLLIKER (A.). Ueber das Ende der Wirbelsäule der Ganoiden und einiger Teleostier. $\quad 4^{\circ}$. Leipzig, 1860.

KÖLLIKER (A.). Icones Histiologicæ.

$4^{\circ}$. Leipzig, $1864 \& 1866$.

KÖLLIKER (A.). Beiträge zur Kenntniss der Polypen. 8. Würzburg, 1870.

KÖLLIKER (A.). Anatomisch-sy:tematische Beschreibung der Alcyonarien. 1. Abtheilung. Die Pennatuliden. $4^{\circ}$. Frankfurt a. M., 1872.

KÖLLIKER (A.). Die normale Resorption des Knochen Gewebes. $4^{\circ}$. Leipzig, 1873.

KÖLLIKER (A.). Die Pennatulide umbellula und zwei neue Typen der Alcyonarien. $\quad 4^{\circ}$. Würzburg, 1874.

KÖLLIKER (A.). Entwicklungsgeschichte des Menschen und der höheren Thiere.

2nd edition. $8^{\circ}$. I Ieipzig, 1880 .

KÖLLIKER (A.). See 'Challenger.'

KÖLLIKeR (A.). See Periodicals: Leipzig.

KÖLLIKER (A.). See RATHKE (H.).

KOLLMAN (A.). Der Tastapparat der Hand der menschlichen Rassen und der Affen.

8․ Hamburg \& Leipzig, 1883. 
KOLLNER (K.). Die geologische Entwickelungsgeschichte der Säugethiere.

Wien, 1882.

KONINCK (L. de). Recherches sur les Animaux fossiles. $4^{\circ}$. Liége, 1847.

KOOLAR (V.). Naturgeschichto der schädlichen Insekten.

8. Wien, 1837.

KOREN (J.). See Sars (M.).

KOREN (J.). See Survers and Explorations : Norway.

KOSSMANN (R.). Die Entonisciden-Studien über Bopyriden. I.-III. 8․ Leipzig, 1881.

KOSSMANN (R.). Zoologische Ergebnisse einer.... Reise in die Küstengebeite des Rothen Meeres.

Pisces. R. Kossmann \& H. Radber.

I. \& II. 4\%. Leipzig, 1877.

Mollusca. H. A. Pagenstecher.

Malacostraca \& Entomostraca. R. Kossurann.

Echinodermata. H. LUDwIG.

KOWALEWSKY (A.). Anatomie des Balanoglossus, delle Chiaje.

$4^{\circ}$. St. Petersburg, 1866.

KOWALEWSKY (A.). Beiträge zur Anatomie und Entwickelungsgeschichte des Loxosoma neapolitanum.

$4^{\circ}$. St. Petersburg, 1866.

KOWALEWSKY (A.). Entwickelungsgeschichte der einfachen Ascidien. $\quad 4^{\circ}$. St. Petersburg, 1866.

KOWALEWSKY (A.). Entwickelungsgeschichte der Rippenquallen.

$4^{\circ}$. St. Petersburg, 1866.

KOWALEWSKY (A.). Beiträge zur Entwickelungsgeschichte der Holothurien. $\quad 4^{\circ}$. St. Petersburg, 1867.

KOWALEWSKY (A.). Entwicklungsgeschichte des Amphioxus lanceolatus. $4^{\circ}$. St. Petersburg, 1867.

KOWALEWSKY (A.). Embryologische Studien an Würmern und Arthropoden. $\quad 4^{\circ}$. St. Petersburg, 1871. 
KOWALEWSKY (W.). Sur l'Anchitherium aurelianense, Cuv., et sur l'histoire paléontologique des Chevaux.

$4^{\circ}$. St. Petersburg, 1873.

KRAATZ (G.). Die Staphylinen-Fauna von Ostindien, insbesondere der Insel Ceylon.

$8^{\circ}$. Berlin, 1859 .

KRAATZ (G.). Revision der Tenebrioniden.

$8^{\circ}$. Berlin, 1865.

KRAATZ (G.). See Periodicals: Berlin.

KRAEPELIN (K.). Ueber die Geruchsorgane der Gliederthiere. 4․ Hamburg, 1833.

KRAUSE (W.). Die Anatomie des Kaninchens.

2nd edition. $8^{\circ}$. Leipzig, 1884.

KRAUSS (C. F. F.). Die Corallineen und Zoophyten der Südsee. $4^{\circ}$. Stuttgart, 1837.

KRAUSS (F.). Die südafrikanischen Crustaceen.

$4^{\circ}$. Stutigart, 1843.

KRAUSS (F.). Die südafrikanischen Mollusken.

$4^{\circ}$. Stuttgart, 1848.

KREFFT (G.). Catalogue of Mammalia in the Australian Museum.

See Museum (A ustralian).

$16^{\circ}$. Sydney, 1864.

KREFFT (G.). On the Vertebrata of the Lower Murray and Darling; and on the Snakes of Sydney.

80. Sydney, 1865.

KREFFT (G.). Australian Vertebrata, recent and fossil.

8. Sydney, $186^{\circ} 7$.

KREFFT (G.). Australian Vertebrata, fossil and recent.

80. Syclney, 1871.

KREFFT (G.). Fossil remains of Mammals, Birds, and Reptiles from the Caves of Wellington Valley.

8. Sydney, 186r. 
KREFFT (G.). Notes on the Fauna of Tasmania.

8.. Sydney, 1868.

KREFFT (G.). The Snakes of Australia.

4\%. Sydney, 1869.

KREFFT (G.). The Mammals of Australia.

fol. Sydney, 1871.

KREFFT (G.). Catalogue of the Minerals and Rocks in the Australian Museum.

See Musedu (Australian).

8. Sydney, 1837.

KROHN (A.). Beiträge zur Entwickelungsgeschichte der Pteropoden und Heteropoden. $4^{\circ}$. Leipzig, 1860.

KRÖYER (H.). Danmarks Fiske.

3 vols. $8^{\circ}$. Kjöbenhavn, 1838-40.

KRÖYER (H.). See Periodicals : Copenhagen.

KRUKENBERG (C. F. W.). Vergleichend-physiologische Studien. 2 vols. $8^{\circ}$. Heidelberg, 1880-82.

KÜCHENMEISTER (F.). Parasiten des Menschen.

2nd edition, by F. A. Z URN. 8. Leipzig, 1855.

8.. Leipzig, 1882.

KUHL (H.). Die Deutschen Fledermäuse.

$4^{\circ}$. Hanau, 1817.

KUHL (H.). Conspectus Psittacorum. 4\% . Bonnce, 1820.

KUHL (H.). Beiträge zur Zoologie und Vergleichenden Anatomie. $\quad 4^{\circ}$. Frankfurt am Main, 1820.

KUHNE (W.). See Hermann (L.). Handbuch der Physiologie.

KUNCKEL D'HERCULAIS (J.). Recherches sur l'Organisation et le Développement des Volucelles. (Diptera.)

2 vols. in 1. $4^{\circ}$. Paris, 1875-81.

KUNTZMANN (J. H. L.). Untersuchungen über den Blutegel.

8. Berlin, 1817. 
KUNZE (G.). Monographia Pselaphorum. Dissertatio entomologica.

80. Lipsiae, 1816.

KUNZE (G.). Entomologische Fragmente.

8. Halle, 1818.

KUPFFER (C.). Die Stammrerwandtschaft zwischen Ascidien und Wirbelthieren.

8. Bonn, 1870.

KÜSTER (H. C.). Icones Molluscorum et Testaceorum.

4․ Nü̈nberg, 1833.

KÜSTER (H. C.). Systematisches Terzeichniss der in der Umgegend Erlangens beobachteten Thiere.

8. Erlangen, 1840.

KÜSTER (H. C.). See Martixi \& Chemritz.

KÜSTER (H. C.) \& KRAATZ (G.). Die Käfer Europas. Parts 1-28. 12 . Nürnberg, 1844-54. Part 29 by G. Kraatz. 12 . Nü̈nberg, 1873.

L.

LABRAMI (D.) \& IMIHOFF (L.). Singulorum generum Curculionidum unam alteramre speciem additis iconibus illustravit.

8. Basel, 1842.

LABRAM (J. D.) \& IMHOFF (L.). Die Schweizerischen Käfergattungen in Abbildungen nach der Natur. Nach Anleitung und mit Text von L. Irrmofr.

$12^{\circ}$. Basel, 1848-52.

LACAZE-DUTHIERS (H.). Histoire Naturelle du Corail. 8. Paris, 1864.

LACAZE-DUTHIERS (H. de). Archives de Zoologie. [In progress.]

8. Paris, 1872-83.

LACEPEDE (B. G. Comte de). Histoire Naturelle des Quadrupèdes ovipares et des Serpens.

2 rols. $4^{\circ}$. Paris, 1788-89. 
LACEPEDE (B. G. Comte de). Histoire Naturelle des Poissons. 5 vols. $4^{\circ}$. Paris, 1798-1805.

LACEPEDE (B. G. Comto de). Histoire Naturelle des Cétacés. 4\%. Paris, 1804. Another edition. 2 vols. $16^{\circ}$. Paris, 1809.

LACÉPẼDE (B. G. Comte de). See Buffon.

LACORDAIRE (T.). Introduction à l'Entomologie. 2 vols. \& Atlas. $8^{\circ}$. Paris, 1834-38.

LACORDAIRE (T.). Monographie des Erotyliens.

8. Paris, 1842.

LACORDAIRE (T.). Monographie des Coléoptères, famillo des Phytophages.

2 vols. $8{ }^{\circ}$. Paris, $1845-48$.

LACORDAIRE (T.). Genera des Coléoptères.

12 vols. $8^{\circ}$. Paris, 1854-76.

LACROIX (A.). Catalogue raisonné des Oiseaux.

80. Toulouse \& Paris, 1873-75.

LA FERTÉ-SÉNECTĖRE (F. de). Monographie des Anthicus et genres roisins. 8. Paris, 1848.

LA FRESNAYE (Baron de). Catalogue des Oiseaux de sa Collection.

8. Paris, 1863.

LAICHARTING (J. N. E. v.). Verzeichniss und Beschreibung der Tyroler-Insecten.

2 vols. in 1. $8^{\circ}$. Ziürich, 1781-84.

LAING (S.) \& HUXLEY (T. H.). Prehistoric Remains of Caithness. With Notes on the Human Remains.

8. London, 1866.

LAISHLEY (R.). A Popular History of British Birds' Eggs. sm. $4^{\circ}$. London, 1858.

LALLEMANT (C.). See Agneli (Dr.). 
LAMARCK (J. B. de). Histoire naturelle des Animaux sans vertèbres. Vols. VI. \& VII. (Mollusca.)

8.. Paris, 1819-22.

2nd edition. 11 vols. $8^{\circ}$. Paris, 1835-45.

LAMARCK (J. B. de). Coquilles fossiles des environs de Paris. $4^{\circ}$. Paris, 1823.

LAMARCK (J. B. de). Genera of Shells, translated by J. P. Children.

8.. London, 1834.

LAMARCK (J. B. de). Philosophie Zoologique. New edition, by Charles Martin. 2 vols. $8^{\circ}$. Paris, 1873.

LAMBERT (E.). Noureaux éléments d'Histoire naturelle. Zoologie.

$12^{\circ}$. Paris, 1865.

LAMONT (J.). Yachting in the Arctic Sens.

8. London, 1876.

LAMOUROUX (J. V. F.). Histoire des Polypiers Coralligènes flexibles.

8.. Caen, 1816.

LAMOUROUX (J. V. F.). Exposition Méthodique des genres de l'ordre des Polypiers. $\quad 4^{\circ}$. Paris, 1821.

LAMOUROUX (J. V. F.). Corallina.

80. London, 1824.

LANDSBOROUGH (D.). A Popular History of British Zoophytes, or Corallines. $\quad$ sm. $4^{\circ}$. London, 1852.

LANDSEER (T.). Characteristic Sketches of Animals, with descriptive and illustrative Notices by J. H. BaRkow.

fol. London, 1832.

LANGGUTHIO (C. A.). De Bestiis Agyptorum Studio conversis in Mumias. $\quad \mathrm{sm} .4^{\circ}$. Vitebergice, 1808.

LANGKAVEL (B.). Scholien zu Aristoteles Werk "De partibus Animalium." $\quad 4^{\circ}$. Berlin, 1863.

LANGLEY (E. A.). Narrative of a Residence at the Court of Meer Ali Moorad; with Wild Sports in the Valley of the Indus. $\quad 2$ rols. $8^{\circ}$. London, 1860. 
LANG (H. G.). Verzeichniss seiner Schmetterlinge.

$12^{\circ}$. Augsburg, 1789.

LANKESTER (E.). Memorials of John Ray.

See Ray Society.

8.. London, 1846.

LANKESTER (E. Ray). See FrsherIes : London. International Fisheries Exhibition, 1883.

LAPEIROUSE (P. de). De novis quibusdam Orthoceratitum et Ostracitum speciebus dissertatiuncula.

[Additional title in French.] $\quad 4^{\circ} . \quad$ Erlanga, 1781.

LAPLACE ( ? ). See ' Fa vorite.'

LAPORTE (F. L. de) \& GORY (H.). Histoire Naturelle des Insectes Coléoptères (Carabidae, Buprestidae, et Clytidce).

8 vols. $8^{\circ}$. Paris, 1837-41.

LAPORTE (F. I. de). Études Entomologiques.

8. Paris, 1834-35.

LARSEN (A.). See Dictionaries.

LASPEYRES (J. H.). Sesiæ Europææ.

$4^{\circ}$. Berolini, 1801.

LATHAM (J.). A General Synopsis of Birds.

7 vols. $4^{\circ}$. London, 1781-87.

LATHAM (J.). A general History of Birds.

10 vols. $4^{\circ}$. Winchester, 1821-24. Index. 1828.

LATHAM (J.). Systema Ornithologiæ, sive Index Ornithologicus. Ed. E. Johanneav. 120. Paris, 1809.

LATHAM (J.) \& DAVIES (H.). Faunula Indica, id est Catalogus Animalium Indiæ orientalis. Ed. J. R. Forster. fol. Haloe, 1795.

LATHAM (R. G.). The Natural History of the Varieties of Man.

$8^{\circ}$. London, 1850. 
LATHAM (R. G.) \& FORBES (E.). The Natural History of the Crystal Palace.

16. London, 1854.

LATHAM (S.). Falconry: or the Faulcon's Lure and Cure: In two Bookes. 1st edition. sm. $4^{\circ}$. London, 1615-18.

LATREIllE (P. A.). See Curier (G.).

LATREILLE (P. A.). Genera et Crustaceorum et Insectorum.

4 vols. $8^{\circ}$. Paris, 1806-9.

LATREILLE (P. A.). Histoire naturelle des Crustacés et des Insectes.

14 vols. 8o. Paris, 1808.

LATREILLE (P. A.). Considérations générales des Crustacés, des Arachnides et des Insectes. $\quad 8^{\circ}$. Paris, 1810.

LATREILLE (P. A.). Familles naturelles du Règne Animal. $8^{\circ}$. Paris, 1825.

LATREILLE (P.A.). Cours d'entomologie ou de l'histoire naturelle des Crustacés, des Arachnides, des Myriapodes et des Insectes.

8.. Paris, 1831.

LATZEL (R.). Die Myriopoden der österreichisch-ungarischen Monarchie.

$8^{\circ}$. Wien, 1880.

LAUGIER DE CHARTROUSE (Le Baron M.). Catalogue des Oiseaux.

4. Arles, 1836.

LAUGIER DE CHARTROUSE (Le Baron M.). See TemMINCK (C. J.).

LAURENT (L.). Recherches sur l'Hydre et l'Éponge d'Eau douce.

8. Paris, 1844.

LAURENT (L.). See ' Fatorite.'

LAURENTI (J. N.). Specimen Medicum, exhibens Synopsis Reptilium.

8․ Vienno, 1768.

LAY (G. L.). See ' Blossom.' 
LAYARD (E. L.). The Birds of South Africa.

8. Cape Town, 1867.

LEA (H. C.). Catalogue of the Tertiary Testacea of the United States. $\quad 8^{\circ}$. Philadelphia, 1848.

LEA (I.). A Synopsis of the Family of Naiades.

4\%. Philadelphia, 1838. 3rd edition. $4^{\circ}$. Philadelphia, 1852.

LEA (I.). Observations on the Genus Unio.

13 vols. \& Indices. 4\%. Philadelphia, 1832-74.

LEA (I.). On a Fossil Saurian of the new Red Sandstone Formation of Pennsylvania. $4^{\circ}$. Philadelphia, 1852.

LEA (I.). A Synopsis of the Family Unionidee. 4th edition. $4^{\circ}$. Philadelphia, 1870.

LEA (J.). See Sirithsonian Miscellaneous Collections.

LEACH (W. E.). The Zoological Miscellany. 3 vols. $8^{\circ}$. London, 1814-17.

LEACH (W. E.). Malacostraca Podophthalmata Britanniæ. $4^{\circ}$. London, 1815.

LEACH (W. E.). Systematic Catalogue of the Indigenous Mammalia and Birds in the British Museum.

8. London, 1816.

LEACH (W. E.). A Synopsis of the Mollusca of Great Britain.

8. London, 1852.

LEACH (W.E.). See Periodicals : London. Willughby Society.

LEAR (E.). Illustrations of the family of Psittacidce or Parrots. fol. London, 1832.

LEAR (E.). See Sowerby (J. de C.).

LEBERT (H.). Verzeichniss Schlesischer Spinnen, mit Aufzählung der Schlesischen Myriapoden.

8. Tübingen, 1875. 
LEBERT (H.). Bau und Leben der Spinnen.

$4^{\circ} . \quad$ Berlin, 1878.

LEBLOND (C.). Matériaux pour servir à l'histoire des Filaires.

8. Paris, 1836.

LEBLOND (C.). Quelques Observations d'Helminthologie.

8. Paris, 1837.

LECHE (J.). Disputatio de Mumia Egyptiaca.

$4^{\circ}$. Londini, 1739.

LE CLERC (G. L. Comte de Buffon). See Buffon.

LE CONTE (J. L.). Revision of the Cicindelce of the United States. 4. Philadelphia, 1856.

LE CONTE (J. L.). Classification of the Coleoptera of North America. $\quad$ Part I. $8^{\circ}$. Washington, 1861. See Suithronian Miscellaneous Collections.

LE CONTE (J. L.). List of the Coleoptera of North America. See Suithisonian Miscellaneous Collections.

LE CONTE (J. L.). New species of North American Coleoptera. 2 parts. $8^{\circ}$. Washington, 1863-73. See Suithsonian Miscellaneous Collecrions.

LE CONTE (J. L.). Say's Description of the Insects of North America. See $\mathrm{SAY}_{\mathrm{I}}(\mathrm{T}$.$) .$

LE CONTE (J. L.). The Coleoptera of Kansas and Eastern New Mexico. $\quad 8^{\circ}$. Washington, 1860.

LE CONTE (J. L.) \& HORN (G. H.). Classification of the Coleoptera of North America. $8^{\circ}$. Washington, 1883.

LE CONTE (J. T.). See Surveys \& Explorattons: WASHINGTON.

LEDERER (J.). Die Noctuinen Europa's.

8. Wien, 1857. 
LEDERER (J.) \& MILLER (L.). See Periodicala : VIENYa.

LEE (H.). See Fisheries : London. International Fisheries Exhibition, 1883.

LEEUWENHOEK (А. จ.). Arcana Naturæ.

$4^{\circ}$. Delphis Batav., 1695.

LEGGE (W. V.). Birds of Ceylon.

3 vols. $4^{\circ}$. London, 1878-80.

LEGRAND (W.). Catalogue of Tasmanian Land-Shells. 8․ Hobart Town, 1870.

LEIDY (J.). Cretaceous Reptiles of the United States. 40. Philadelphia, 1864.

LEIDI (J.). Freshwater Rhizopods.

$4^{\circ}$. Washington, 1879.

See Strieys \& Explorations: U.S., Washington.

LE KEUX (J.). Illustrations of Natural History.

8. London, 1844.

LENBCKE (G.). See Tedtsche Orithologie.

LENZ (H.). Die wirbellosen Thiere der Travemünder Bucht. Part I. $4^{\circ}$. Berlin, 1875.

[In progress.]

LENZ (H.). Die anthropomorphen Affen des Lübecker IIuseums.

4. L Lübeck, 1876.

LENZ (H. O.). Zoologie der alten Griechen und Römer.

$8^{\circ}$. Gotha, 1856.

LEOPOLdO (MI.). See Periodicals: Milan.

LÉOTAUD (A.). Oiseaux de l'île de la Trinidad.

8.. Port d'Espagne, 1866.

Lepeletier (A.). See Saint Fargead. 
LEPRIEUR (C. E.). Notes sur le genre Homonia.

8. Colmar, 1870.

LEREBOULLET (A.). Recherches d'Embryologie Comparée, sur le développement du Brochet, de la Perche et de l'Ecrevisse. $4^{\circ}$. Paris, 1862.

LESKE (N. G.). Ichthyologiæ Lipsiensis Specimen.

8.. Lipsice, 1774.

LESLIE (G.) \& HERDMAN (W. A.). The Invertebrate Fauna of the Firth of Forth.

8. Edinburgh, 1881.

LESSER (F. C.). Theologie des Insectes. Traduit de l'Allemand par P. LYONNET.

2 vols. $8^{\circ}$. La Haye, 1742.

LESSON (R. P.). Manuel de Mammalogie.

$16^{\circ}$. Paris, 1827.

LESSON (R. P.). Histoire Naturelle des Phoques.

8. Paris, 1828.

LESSON (R. P.). Histoire Naturelle générale et particulière des Mammifères et des Oiseaux.

10 vols. $8^{\circ}$. Paris, 1828-36.

[Complément des Eurres de Buffon.]

LESSON (R. P.). Histoire naturelle des Oiseaux.

8. Paris, 1829.

LESSON (R. P.). Histoire naturelle des Oiseaux-Mouches. $8^{\circ}$. Paris, 1829-30.

LESSON (R. P.). Centurie zoologique. $\quad 4^{\circ}$. Paris, 1830.

LESSON (R. P.). Illustrations de Zoologie.

8. Paris, 1831.

LESSON (R. P.). Traité d'Ornithologie.

2 vols. $8^{\circ}$. Paris, 1831.

LESSON (K. P.). Histoire naturelle des Colibris.

8․ Paris, 1831-32. 
LESSON (R. P.). Les Trochilidées ou les Colibris.

8०. Paris, 1832 .

LESSON (R. P.). Histoire naturelle des Oiseaux de Paradis et des Épimaques.

8․ Paris, 1835.

LESSON (R. P.). Histoire naturelle générale. Mammifères.

$8^{\circ}$. Paris, 1836.

LESSON (R. P.). Histoire naturelle des Zoophytes. Acalèphes. Texte et Atlas. $8^{\circ}$. Paris, 1843.

LESSON (R. P.). Description de Mammifères et d'Oiseaux récemment découverts, précédée d'un tableau sur les. races humaines.

$12^{\circ}$. Paris, 1847.

LESSON (R. P.). See Belanger (C.).

LESSONA (M.). Nota intorno al Genere Tropidonotus, Kuhl, ed alle sue specie in Piemonte. $8^{\circ} . \quad$ Torino, 1877.

LESSONA (MI.). Studii sugli Anfibii anuri del Piemonte. $4^{\circ}$. Roma, 1877.

LESSONA (M.). Nota intorno al Tempo della riproduzione della Vipera aspis, L.

$8^{\circ}$. Torino, 1880.

LeSsona (M.). See Periodicals : Genoa and Modena. Archivio per la Zoologia.

LETHIERRY (L.). Catalogue des Hémiptères du Département du Nord. 2nd edition. $8^{\circ}$. Lille, 1874.

LEUCKART (F. S.). Zoologische Bruchstücke.

$4^{\circ}$. Helmstädt, 1819.

LEUCKART (F. S.). Versuch einer naturgemässen Eintheilung der Helminthen.

8․ Heidelberg \& Leipzig, 1827.

LEUCKART (F. S.). Breves animalium marinorum descriptiones.

$4^{\circ}$. Heidelbergoe, 1828.

LEUCKART (F. S.). De rariori et singulari animalium vertebratorum habitu.

$4^{\circ}$. Heidelberga, 1832. 
LEUCKART (F. S.). Untersuchungen ueber die ausseren Kiemen der Embryonem von Rochen und Hayen.

$8^{\circ}$. Stuttgart, 1836.

LEUCKART (F. S.). Untersuchungen über das Zwischenkieferbein des Menschen.

$4^{\circ}$. Stuttgart, 1840.

LEUCKART (F. S.). Observationes Zoologicæ de Zoophytis Coralliis, speciatim de genere Fungia.

$4^{\circ}$. Friburgi-Brisigavorum, 1841.

LECCKART (F. S.). Zoologische Bruchstücke. III. Helminthologische Beiträge. $\quad 4^{\circ}$. Freiburg, 1842.

LEUCKART (R.). Ueher die Morphologie und die Verwandtschaftsverhältnisse der wirbellosen Thiere.

8. Braunschweig, 1848.

LEUCKART (R.). Ueber den Polymorphismus der Individuen.

$4^{\circ}$. Giessen, 1851.

LEUCKART (R.). Bau und Entwickelungsgeschichte der Pentastomen.

$4^{\circ}$. Leipzig \& Heidelberg, 1860.

LEUCKART (R.). Untersuchung über Trichina spiratis. Zugleich ein Beitrag zur Kenntniss der Wurmkrankheiten. 4․ Leipzig \& Heidlelberg, 1860.

LEUCKART (R.). Die menschlichen Parasiten und die von ihnen herrührenden Krankheiten.

2 vols. $8^{\circ}$. Leipzig \& Heidelberg, 1863-76.

LEUCKART (R.). De statu et embryonali et larvali Echinorhynchorum eorumque metamorphosi.

$4^{\circ}$. Lipsice, 1872.

LEUCKART (R.). Decani commentatio "de Zoophytorum et historia et dignitate systematica" patrio sermone conscripta.

$4^{\circ}$. Lipsice, 1873.

LEUCKART (R.). Allgemeine Naturgeschichte der Parasiten. $\quad 8^{\circ}$. Leipzig \& Heidelberg, 1879. 
LEUCKART (R.). Die Parasiten des Menschen.

Band I. Lief. 1, 2. $\quad 8^{\circ}$. Leipzig \& Heidelberg, 1879-81.

LEUCKART (R.). Ueber Bastardfische.

8. Berlin, 1882.

LEUCKART (R.). See Bergmann (E.).

LEUCKART (R.) \& NITSCHE (H.). Zoologische Wandtafeln. $\quad 4^{\circ} \&$ fol. Cassel, 1877.

LEUCKART (R.) \& RATHKE (H.). Beiträge zur Entwicklungsgeschichte der Hirudineen von H. RathkE, herausgegeben von R. Lебска RT. $\quad 4^{\circ}$. Leipzig, 1862.

LEUE (S. F.). De Pleurobranchcea novo Molluscorum genere.

$4^{\circ}$. Halae, 1813.

LEUTHNER (F.). Die Mittelrheinische Fischfauna. 8. Basel, Genf, \& Lyon, 1877.

LEVAILLANT (F.). Histoire Naturelle des Oiseaux d'Afrique. 2 vols. $12^{\circ}$. Paris, 1798.

LEVAILLANT (F.). Histoire naturelle des Oiseaux d'Afrique. 6 vols. $4^{\circ}$. Paris, 1799-1808.

LEVAILLANT (F.). Histoire Naturelle d'une partio d'Oiseanx nouveaux et rares de l'Amérique et des Indes.

fol. Paris, 1801.

LEVAILLANT (F.). Histoire naturelle des Oiseaux de Paradis et des Rolliers suivie de celle des Toucans et des Barbus. 2 vols. fol. Paris, 1806.

LEVAILLANT (F.) \& SAINT-HILAIRE (A. B.). Histoire naturelle des Perroquets.

Vols. I. \& II. fol. Paris, 1801. Vol. III. fol. Paris, 1837-38.

LEVER (Sir Ashton). A short History of Insects,.... A companion to those who visit the Leverian Museum.

12. Norwich [1797?]. 
LEVESON (H. A.). Sport in Many Lands. By H. A. L. "The Old Shekarry." 2 vols. $8^{\circ}$. London, 1877.

LEVI (Leone). See Fisheries : Loxpor. International Fisheries Exhibition, 1883.

LEWIN (j. W.). A Natural History of the Lepidopterous Insects of New South Wales. $\quad 4^{\circ}$. London, 1822.

LEWIN (J. W.). Natural History of the Birds of New South Wales. fol. London, 1822. Another edition. $4^{\circ}$. London, 1838.

LEWIN (W.). The Birds of Great Britain, with their Eggs. 7 vols. $4^{\circ}$. London, 1789-94.

LEWIN (W.). The Papilios of Great Britain. Les Papillons de la Grande Brétagne. $\quad 4^{\circ}$. London, 1795.

LEWIS (E. J.). The American Sportsman. roy. $8^{\circ}$. Philadelphia, 1855.

LEWIS (G.). A Catalogue of Coleoptera from the Japanese Archipelago.

8. London, 1879.

LEWIS (W. A.). A Discussion of the Law of Priority in Entomological Nomenclature.

8. London, 1872.

LEWNSOHN (L.). Die Zoologie des Talmuds.

8. Frankfurt, 1858.

LEYDIG (F.). Beiträge zur mikroskopischen Anatomie und Entwickelungsgeschichte der Rochen und Haie.

8. Leipzig, 1852.

LEYDIG (F.) Anatomisch-histologische Untersuchungen über Fische und Reptilien.

$4^{\circ}$. Berlin, 1853.

LEYDIG (F.). Lehrbuch der Histologie des Menschen und der Thiere.

8. ${ }^{\circ}$ Hamm, 1857.

LEYDIG(F.). Naturgeschichte der Daphniden (Crustacea cladocera).

$4^{\circ}$. Tübingen, 1860. 
LEYDIG (F.). Vom Bau des thierischen Körpers. Handbuch der vergleichenden Anatomie.

$8^{\circ} \&$ obl. fol. Trübingen, 1864.

LEYDIG (F.). Skizze zu einer Fauna Tubingensis.

8. Stuttgart, 1867.

LEYDIG (F.). Die in Deutschland lebenden Arten der Saurier.

$4^{\circ}$. Tübingen, 1872.

LEYDIG (F.). Die Anuren Batrachier der deutschen Fauna.

8. Bonn, 1877.

LEYDIG (F.). Die augenähnlichen Organe der Fische.

$8^{\circ}$. Bonn, 1881.

LEYDIG (F.). Untersuchungen zur Anatomie und Histologie der Thiere.

8. Bonn, 1883.

LICHTENSTEIN (A. A. H.). Commentatio philologica de Simiarum... formis.

8. Hamburgi, 1791.

LiChTENSTEIN (A. A. H.). See Periodicals: London. Willughby Society.

LICH'TENSTEIN (H.). Ueber die Stachelratten, die Gattung Dendrocolaptes und die Sepien mit Krallen.

$4^{\circ}$. Berlin, 1820.

LICHTENSTEIN (H.). Verzeichniss der Doubletten des zoologischen Museums zu Berlin.

$4^{\circ}$. Berlin, 1823.

LICHTENSTEIN (H.). Darstellung neuer Säugethiere.

fol. Berlin, 1827-34.

LICHTENSTEIN (H.). Nomenclator Avium.

8. Berlin, 1854.

LICHTENSTEIN (H.). Nomenclator Reptilium et Amphibiorum Musei Zoologici Berolinensis. $\quad 8^{\circ}$. Berlin, 1856.

LICHTENSTEIN (H.). Ueber einige nordamerikanische Hirsch-Arten.

$4^{\circ}$. Berlin, 1857. 
LICHTENSTEIN (H.). See Forster (J. R.).

LICHTENSTEIN (H.) \& PETERS (W.). Ueber neue merkwürdige Säugethiere. $4^{\circ}$. Berlin, 1855.

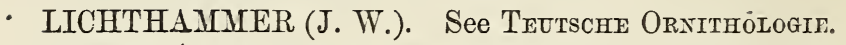

LIDTH DE JEUDE (T. G. ..). Recueil de figures des vers intestinaux.

obl. 4\%. Leyde, 1829.

LIDTH DE JEUDE (T. W. van). De spijsverterings organen der Phytophage Lamellicornienlarven.

8.. Utrecht, 1882.

LIER (J. van). Verhandeling over de Drentsche Slangen en Adders. $\quad 4^{\circ}$. Amsterdam, 1781.

LILLJEBORG (W.). Systematisk öfversigt af de gnagande däggdjuren, Glires.

$4^{\circ}$. Upsala, 1866.

LILLJEBORG (W.). Sveriges och Norges Ryggradsdjur. 2 vols. $8^{\circ}$. Upsala, 1870.

LILLJEBORG (W.). Sveriges och Norges Fiskar.

Första häftet. $8^{\circ}$. Upsala, $18 \$ 1$.

LILLJEBORG (W.). See Periodicals: London. Ray Society.

LINCK (H. E.). Die Schlangen Deutschlands.

8. Stuttgart, 1855.

LINDAHL (J.). Om Pennatulid-slägtet Umbellula.

$4^{\circ}$. Stockholm, 1874.

LINDEMAN (M.). See Fisheries : Berlin.

LINDEN (P. L. V.). Monographiæ Libellulinarum Europæarum Specimen.

$8^{\circ}$. Bruxellis, 1825.

LINDERMAYER (K. A.). Die Vögel Griechenlands, ein Beitrag zur Fauna dieses Landes. $\quad 8^{\circ}$. Passau, 1860. 
LINN EUS (C.). Fauna Suecica.

$8^{\circ}$. Lugduni Batavorum, 1746. Another edition. $8^{\circ}$. Stockholmice, 1761.

LINNAUS (C.). Fauna Suecica. Entomologia.

Ed. Villers. 4 vols. \& Atlas. $8^{\circ}$. Lugduni, 1789.

LINN EUS (C.). Systema Naturæ.

8. Lugduni Batavorum, 1756. Another edition (12th). 3 vols. 80. Holmice, 1766-68. Another edition (13th). Cura J. F. Guelin.

8 vols. Lugduni, 1788-96.

LINN AUS (C.). Museum Ludoricæ Ulricæ Reginæ.

8. Holmiae, 1764.

LINN AUS (C.). Fundamenta Testaceologiæ.

$4^{\circ}$. Upsatice, 1771.

LINSTOW (O. von). Compendium der Helminthologie.

8. Hannover, 1878.

LINZ (J. M.). See InseKtengeschichte.

LIPP (F. J.). De Piscibus Venenatis. Sectio Prima. 8.. Tubinga, 1829.

LISCHKE (C. E.). See Novitatrs Conchologicæe.

LISTER (M.). Historiæ animalium Angliæ Tres tractatus; unus de Araneis; alter de Cochleis tum terrestribus tum fluviatilibus; tertius de Cochleis Marinis.

$4^{\circ}$. Londini, 1678.

LISTER (M.). Exercitatio Anatomica, in qua de Cochleis . . . agitur. $\quad 8^{\circ}$. Londini, 1694.

LISTER (M.). Exercitatio anatomica altera, in qua maximè agitur de Buccinis fluviatilibus et marinis.

8. Londini, 1695.

LISTER (M.). Conchyliorum Bivalvium utriusque Aquæ Exercitatio Anatomica Tertia. $\quad 4^{\circ}$. Londini, 1696. 
LISTER (M.). Historiæ sive Synopsis methodicæ Conchyliorum et Tabularum anatomicarum editio altera.

fol. Oxonii, 1770.

LISTER (M.). See Goedartios (J.).

LJUNGMAN (A.). Ophiuroidea viventia hucusque cognita. (With MS. additions.) $\quad 8^{\circ}$. Holmiae, 1867.

LJUNGMAN (A. V.). Preliminär Berättelse om Sillfisket [Herring-fishing] 1873-75. $\quad 8^{\circ}$. Upsala, 1874-75.

LLOYD (L.). Field Sports of the North of Europe.

2 vols. $8^{\circ}$. London, 1831.

LLOYD (L.). Scandinavian Adventures.

2nd edition. 2 vols. $8^{\circ}$. London, 1854.

LLOYD (L.). The Game Birds and Wild Fowl of Sweden and Norway.

8. London, 1867.

LLOYD (L.). See Anderssun (C. J.).

LOCARD (A.). Malacologie Lyonnaise.

8․ Lyon, 1877.

LOCARD (A.). Description de la Faune Malacologique des terrains quaternaires. $\quad 8^{\circ}$. Lyon \& Paris, 1879.

LOCARD (A.). Nouvelles Recherches sur les Argiles Lacustres de Lyon. $\quad 8^{\circ}$. Lyon \& Paris, 1880.

LOCARD (A.). Catalogue Général des Mollusques vivants de France.

$8^{\circ}$. Lyon \& Paris, 1882.

LOCHE (Capt.). Catalogue des Mammifères et des Oiseaux observés en Algérie.

80. Paris, 1858.

IOCHE (Capt.). See Surveys \& Explorations : France.

LOCKE (J.). Tweed and Don. $\quad 8^{\circ} . \quad E d i n b ., 1860$.

LOEW (F.). See Brader (F.). 
LOEW (H.). Die Dipteren-Fauna Südafrika's.

$4^{\circ}$. Berlin, 1860.

LOEW (H.). Diptera Americæ septentrionalis indigena.

2 parts in 1 vol. $8^{\circ}$. Berolini, 1861-72.

LOEW (H.). Die Europäischen Bohrfliegen (Trypetidce). fol. Wien, 1862.

LOEW (H.). Monographs of the Diptera of North America. $\quad$ Parts I. \& II. $8^{\circ}$. Washington, 1862-64.

See Sirithsonian Miscellaneous Collections.

LOEW (H.). Beschreibung Europäischer Dipteren.

Theil I.-III. $8^{\circ}$. Halle, 1869-73.

LOEWE (L.). Beiträge zur Anatomie und zur Entwickelungsgeschichte des Nervensystems der Säugethiere und des Menschen.

$4^{\circ}$. Berlin, 1880.

LONGET (F. A.). Anatomie et Physiologie du Système nerveux de l'homme et des animaux vertébrés.

2 vols. $8^{\circ}$. Paris, 1842.

LORD (W. B.). Crab, Shrimp, and Lobster Lore.

$12^{\circ}$. London, 1867.

LORENT (J. A.). De animalculis infusoriis.

$4^{\text {o. }}$ Mannhemiis, 1837.

LORENZEN (E.). See Steenstrup (J. J. S.).

LORI (F. A.). See Siebold (C. T. von).

LOUdON (J. C.). See Perionicals: London. Magazine of Natural History.

LOVÉN (N. H.). See KINBerg (J. G. H.).

LOVÉN (S.). Index Molluscorum litora Scandinaviæ habitantium.

8․ Holmice, 1846.

LOVÉN (S.). Om utvetlingen hos slägtet Chiton.

$8^{\circ}$. Stockholm, 1855. 
LOVEN (S.). Etudes sur les Échinoïdées.

4\%. Stockholm, 1874-75.

LOW (D.). The Breeds of the Domestic Animals of the British Islands.

2 vols. $4^{\circ}$. London, 1842.

LOW (G.). Fauna Orcadensis. $\quad 4^{0} . \quad$ Edinburgh, 1813.

LOWE (E. J.). The Conchology of Nottingham.

8. London, 1853.

LOWE (R. T.). Description d'un nouveau genre de Poisson de la famille des Murénoïdes, rapporté de Madère.

$4^{\circ}$. St. Petersburg, 1851.

LOWE (R. T.). A History of the Fishes of Madeira.

$8^{\circ}$. London, 1860.

LOWNDES (W. T.). The Bibliographer's Manual of English Literature. 4 vols. $8^{\circ}$. London, 1834.

LOWNE (B. T.). See Museum: College of Surgeons.

LUBBOCK (Sir J.). A Monograph of the Collembola and Thysanura. 8. London, 1873.

See $R_{A Y}$ Societr.

LUBBOCK (Sir J.). The Origin of Civilisation and the Primitive Condition of Man.

3rd edition. $8^{\circ}$. London, 1875.

LUBBOCK (Sir J.). Ants, Bees, and Wasps, a record of observations on the Habits of the Social Hymenoptera.

3rd edition. $8^{\circ}$. London, 1882.

LUBBOCK (R.). Observations on the Fauna of Norfolk.

$8^{\circ}$. Norwich, 1845.

LUCAS (H.). Histoire Naturelle des Lépidoptères exotiques.

$8^{\circ}$. Paris, 1881 ?

LUCAS (H.). See De Laporte (F. L. Comte de Castelnau).

LUCAS (H.). See Surveys \& Explorations: France. 
LUCHSINGER (B.). See Heruand (L.). Handbuch der Physiologie.

LUDWIG (H.). Morphologische Studien an Echinodermen. 8. Leipzig, 1877-79.

LUDWIG (H.). See Kossuann (R.).

LUND (P. W.). Blik paa Brasiliens Dyreverden.

Text and Atlas. 2 vols. $4^{\circ}$. Kjöbenhavn, 1838.

LUNDBERG (R.). See Fisheries: BerLin. Internationale Fischerei-Ausstellung, 1880.

LUNEL (G.). Histoire Naturelle des Poissons du Bassin du Léman.

8.. Genève, Bale, Lyon, 1874.

IÜTKEN (C. F.). Oversigt over Grönlands Echinoctermata.

8. Kjöbenhavn, 1857.

L ̈̈TKEN (C. F.). Additamenta ad historiam Ophiuridarum.

8․ Kjöbenhavn, 1858-59-69.

LÜTKEN (C. F.). Bidrag til kundskab om Echinoderme. 8. Kjöbenhavn, 1864.

LYMAN (T.). Catalogue of Ophiuridce and Astrophytidce in the Museum of Comparative Zoology at Harvard College.

$4^{\circ}$. Cambridge, Mass., 1865.

Supplement to same. $\quad 4^{\circ}$. Cambridge, Mass., 1871.

See Museum : Harvard College. Memoirs.

LYMAN (T.). Zoological Results of the Hassler Expedition. Ophiuridce and Astrophytidce.

4\%. Cambridge, Mass., 1875.

See Museum : Harvard College. Memoirs.

LYMAN (T.). A Preliminary List of Living Ophiuridce and Astrophytidce. $\quad 4^{\circ}$. Cambridge, Mass., 1880.

LYMAN (T.). A Structural feature in Deep-Sea Ophiurians.

$4^{\circ}$. Boston, 1880.

LYMAN (T.). See 'Challenger.' 
LYONNET (P.). Traité anatomique de la Chenille, qui ronge le bois de Saule. $\quad 4^{\circ}$. La Haye, 1760. Another edition. $4^{\circ}$. La Haye, 1762.

LYONNET (P.). Recherches sur l'anatomie d'Insectes.

$4^{\circ}$. Paris, 1832.

LYONNET (P.). See Lesser (F. C.).

M.

II. (G.). OpuscoIo sul Grillo-Talpa. $\quad 4^{\circ}$. Roma, 1839.

MAASSEN (J. P.). Beiträge zur Schmetterlingskunde.

Parts I.-III. 4 ${ }^{\circ}$. Elberfeld, 1869.

McANDREW (R.). On the Geographical Distribution of Testaceous Mollusca. 8\%. Liverpoot, 1854.

McCLELLAND (J.). Indian Cyprividae.

$4^{\circ}$. Ccklcuttc, 1839 .

$\operatorname{McCOY}\left(\mathrm{F}_{\circ}\right)$. Prodromus of the Palæontology of Victoria. Decades I.-VII.

8. Melbourne, 1874-82.

MACDONALD (D. G. F.). Grouse Disease; its Causes and Remedies.

$8^{\circ}$. London, 1883.

MACGILLIVRAY (W.). Descriptions of the Rapacious Birds of Great Britain. $\quad 12^{\circ}$. Edinburgh, 1836.

MACGILLIVRAY (W.). A History of British Birds.

5 vols. $8^{\circ}$. London, 1837-52.

MACGILLIVRAY (W.). Manual of British Ornithology. post $8^{\circ}$. London, 1840-42. 2nd edition. post $8^{\circ}$. London, 1846.

MACGILLIVRAY (W.). A History of the Molluscous Animals of the Counties of Aberdeen, Kincardine, and Banff, to which is appended an account of the Cirripedal Animals of the same district. post $8^{\circ}$. London, 1843. 
MACGILLIVRAY (W.). Animals of Scotland.
A History of the Molluscous $8^{\circ}$. London, 1844.

MACGILLIVRAY (W.). The Conchologist's Text-Book. 2nd edition. $12^{\circ}$. Edinburgh \& London, 1845.

MaCGILIIVRaY (T.). See Periodicals : Edinbergh.

MACHADO (A.). Catálogo de las Ares observandas en algunas provincias de Andalucia. roy. $8^{\circ}$. Sevilla, 1854.

IIACHADO (A.). Catálogo de los Peces .... de Cadiz y Huelra, con inclusion de los del Rio Guadalquivir.

Y, Suplemento á mi Catálogo de Ares (1854). roy. $8^{\circ}$. Sevilla, 1857.

MACHADO (A.). Erpetologia Hispalensis seu Catalogus metodicus Reptilium et Amphibiorum in provincia Hispalensi virentium.

$4^{\circ}$. Sevilla, 1859.

MACHADO Y NUÑEZ (A.). Catálogo metódico y razonado de los Mamíferos de Andalucía.

roy. $8^{\circ}$. Sevilla, 1869.

McILRAITH (J.). Life of Sir John Richardson.

8. London, 1868.

McINTOSH (W. C.). A Monograph of the British Annelids. fol. London, 1873-74.

See Ray Society.

McINTOSH (W. C.). The MIarine Invertebrates and Fishes of St. Andrews.

$4^{\circ}$. Edinburgh \& London, 1875.

MACKER (Dr.). See Peyerinhoff (H. de).

McLACHLAN (R.). A Catalogue of British Neuroptera.

8. London, 1870.

McLACHLAN (R.). A Monographic Revision of the Trichoptera of the European Fauna. $8^{\circ}$. London, 1874-78.

McLACHLAN (R.). See Survers \& Explorations : YARKAND. 
MACLEAY (W. S.). Horæ Entomologicæ.

MACLEAY (W. S.). Annulosa Javanica.

$4^{\circ}$. London, 1825.

Another edition. $\quad 8^{\circ}$. Paris, 1833.

MACLEAY (W. S.). A Letter on the dying struggle of the Dichotomous System.

$8^{\circ}$. London, 1830.

MACLISE (J.). Comparative Osteology.

fol. London, 1847.

MACMASTER (A. C.). Notes on Jerdon's Mammals of India.

See Jerdon (T. C.).

8. Madras, 1870.

MACQUART (J.). Histoire naturelle des Insectes. Diptères. 3 vols. $8^{\circ}$. Paris, 1834-36.

MACQUART (J.). Diptères Exotiques.

2 vols. $8^{\circ}$. Paris, 1838-40.

MACQUART (J.). Facultés Intérieurs des Animaux Invertébrés. $\quad 8^{\circ}$. Lille, 1850.

MAGENDIE (F.). See Deswoulins (A.).

' MIAGENTA.' Zoologia del viaggio intorno al globo della R. Piro corvetta 'Magenta' duranti gli anni 1865-68. Crostacei, Brachiuri, e Anomouri per A. TARGIoni-Tozfitr. roy. $8^{\circ}$. Firenze, 1877.

MAGITOT (E.). Traité des Anomalies du Système Dentaire chez l'Homme et les Mammifères. $4^{\circ}$. Paris, 1877.

MagnUs (J.). See Fisheries : BerLin. Internationale Fischerei-Ausstellung, 1880.

MaIllot (E.). See Malpighi (M.).

MAINWARING (F. B. Massey). See Fisheries : London. International Fisheries Exhibition, 1883. 
MAISSIAT (J.). Etudes de Physique Animale.

$4^{\circ}$. Paris, 1843.

MAITLAND (R. T.). Descriptio systematica animalium Belgii. 8. . Lugduni Batavorum, 1851.

MAITLAND (Sir J. R. G.). See Fisheries: London. International Fisheries Exhibition, 1883.

MALHERBE (A.). Faune Ornithologique de la Sicile.

8.. Metz, 1843.

MALHERBE (A.). Faune Ornithologique de l'Algérie.

$8^{\circ}$. Metz, 1855.

MALHERBE (A.). Monographie des Picidées.

4 vols. fol. Metz, 1861-62.

MALM (A. W.). Zoologiska Observationer.

8․ Göteborg, 1853.

Another edition. $8^{\circ}$. Göteborg, 1873.

MALM (A. W.). Om Sjöbruk i allmänhet och lax-afvel Isynnerhet. $8^{\circ}$. Göteborg, 1863.

MALM (A. W.). Bidrag till Kännedom af Pleuronektoidernas.

$4^{\circ}$. Stockholm, 1868.

MALM (A. W.). Hvaldjur i sveriges Museer, år 1869.

$4^{\circ}$. Stockholm, 1871.

MALM (A. W.). Göteborgs och Bohnsläns Fauna, Ryggradsdjuren.

8. Göteborg, 1877.

MALMBERG (A. J.). Fauna Fennica. Suomen Eläimistö Nuorisolle.

8०. Helsingissä, 1872.

MALMGREN (A. J.). Kritisk öfversigt af Finlands FiskFauna.

8. Helsingfors, 1863.

MALPIGHI (M.). Traité du Ver à Soie. Avec une traduction et des notes en Français par E. MaILLot.

$4^{\circ}$. Montpellier, 1878. 
MALPIGHI (M.). Opera omnia Botanico-Medico-Anatomica. 2 vols. $4^{\circ}$. Lugduni Batavorum, 1687.

MALY (R.). See Hermann (L.). Handbuch der Physiologie.

MAMMALIA: Sketches in Natural History: History of the Mammalia (Knight's Monthly Series).

6 vols. in $3.12^{\circ}$. London, 1849.

MAN, The Remote Antiquity of, not proven. By B.C. Y.

$8^{\circ}$. Lonclon, 1882.

MANBY (G. W.). Journal of a Voyage to Greenland in 1821. 2nd edition. $8^{\circ}$. London, 1823.

MANDL (L.). Traité pratique du Microscope.

8. Paris, 1839.

MANDT (M. G.). Observationes in Historiam Naturalem et Anatomiam Comparatam in Itinere Grœnlandico factæ.

8.. Berolini, 1823.

MANGILI (C. G.). Nuove Ricerche Zootomiche sopra alcune specio di Conchiglie Bivalvi. $\quad 8^{\circ}$. Milano, 1804.

MANGIN (A.). See Adams (W. H. D.).

MANLEY (J. J.). See Fisheries : LoNdon. International Fisheries Exhibition, 1883.

MANNERHEIM (C. G.). Eucnemis Insectorum genus Monographice tractatum Iconibusque illustratum.

8. Petropoli, 1823.

MANZONI (A.). Saggio di Conchiologia Fossile Subappennina : Fauna delle Sabbie Gialle. $8^{\circ}$. Imola, 1868.

MARCUSEN (J.). Die Familie der Mormyren.

$4^{\circ}$. St. Petersburg, 1864.

MARIE (E.). Mélanges ornithologiques sur la Faune do la Nouvelle-Calédonie.

$8^{\circ}$. Bordeaux, 1870. 
MARION (A. F.). Recherches zoologiques et anatomiques sur des Nématoides non Parasites, Marins.

8. Paris, 1870 .

MaRK (E. L.). See Mosedr. Hartard College.

MARKHAM (F.). Shooting in the Himalayas.

$8^{\circ}$. London, 1854.

MAROTT (G. P.). Lepidotteri nuovi e rari trovati in Sicilia.

8. Palermo, 1872.

MARSCHALL (A. de). Nomenclator Zoologicus.

8.. Vindobonce, 1873.

MARSEUL (S. M. de). Catalogue des Coléoptères d'Europe.

$12^{\circ}$. Paris, 1857.

MARSEUL (S. M. de). Catalogus Coleopterorum Europx et confinium.

16. Paris \& Londres, 1863.

MARSH (G. P.). The Camel : his Organization, Habits, and Uses.

$12^{\circ}$. Boston, 1856 .

MARSHALL (C. H. T. \& G. F. L.). A Monograph of the Capitonida. $4^{\circ}$. London, 1870-71.

MARSHALL (G. F. L.) \& NICEVILLE (L. de). The Butterflies of India, Burmah, and Ceylon.

Vol. I. Parts 1-2. 8०. Calcutża, 1882-83. [In progress.]

MARSHALL (T. A.). Ichneumonidum Britannicorum Catalogus.

$8^{\circ}$. Londini, 1870.

MARSHALL (T. A.). A Catalogue of British Hymenoptera: Chrysidida, Ichneumonida, Braconida, and Evaniidle.

8.. London, 1872.

The same eontinued: Oxyra. $\quad 8^{\circ}$. London, 1873.

MARSHAM (T.). Coleoptera Britannica.

8. Loncton, 1802 .

MARSIGLI (L. F.). Natuurkundige Beschryving der Zeën.

fol. 'S Graventage, 1786. 
MARsigLI (I. F. Comte de). Histoire Physique de la Mer.

MARSILI (A. F.). Danubius Pannonico Mysicus.

6 vols. fol. Haga Comitum \& Amstelodami, 1726.

MARSILLI (L. F). See Marsigli (L. F.).

MARSTON (R. B.). See Fisheries : London. International Fisheries Exhibition, 1883.

MARTENS (E. von). Conchologische Mittheilungen als Fortsetzung der Novitates Conchologicæ.

Band 1, Heft 1-4. $8^{\circ}$. Cassel, 1880. [In progress.]

MARTENS (E. von). Beiträge zur Meeresfauna der Insel Mauritius und der Seychellen. Mollusken.

See MöвiUs (K.).

$4^{\circ}$. Berlin, 1880.

MARTENS (E. von). Ueber Centralasiatische Mollusken. $4^{\circ}$. St. Pétersbourg, 1882.

MARTENS (E. von). Die Weich- und Schalthiere.

8. Leipzig \& Prag, 1883.

MaRTENs (E. von). See Novitates Conchologice.

MARTENS (E. von). See Surveys \& Explorations : Germany.

MARTENS (E. von). See Von der Decken's Reisen.

MARTENS (E. von) \& LANGKAVEL (B.). Donum Bismarckianum. Eine Sammlung von Sïdsee-Conchylien. $4^{\circ}$. Berlin, 1871.

MARTENS (E.). Viaggio di Spizberga o Gronlanda. 1671. Translated by D. Bertuzzin. $\quad 12^{\circ}$. Bologna, 1680.

MARTIN (C.). See L.MMARCK (J. B.). 
MARTIN (M.). Observations on Marine Vermes, \&c.

$4^{\circ}$. Exeter, 1786.

MARTIN (P. L.). Die Praxis der Naturgeschichte. Atlas von L. Martin und F. Specht.

Text, $8^{\circ}$. Atlas, $4^{\circ}$. Weimar, 1876-82.

MARTIN (W. C. L.). An Introduction to the Study of Birds. $12^{\circ}$. London, n. d.

MARTIN (W. C. L.). A Natural History of Quadrupeds. 8. London, 1840.

MARTIN (W. C. L.). The Ox.

roy. 8०. [London, n. d.]

MARTIN (W. C. L.). A General History of HummingBirds, or the Trochilidce; with reference to the Collection of J. Gould, F.R.S.

8. London, 1852.

MARTINELLI (A.) \& RABBENS (A.). La Legislazione sulla Pesca in Italia. $8^{\circ}$. Torino, 1883.

MARTINEZ Y SAEZ (F.). Distribucion metódica de los Vertebrados.

8. Madrid, 1879.

MARTINI (F. H. W.). Neues Conchylien-Cabinet. 12 vols. in 14. $4^{\circ}$. Nürnberg, 1769.

MARTINI (F. H. W.) \& CHEMNITZ (J. H.). Die Conchylien.

12. Bregenz, 1787.

MARTIN-SAINT-ANGE (G. J.). See BAUdRIMIONT (A.).

MARTIUS (C. F. P. von). See Spix (J. B. von).

MARTORELL Y PEÑA (M.). Catálogos Sinonimicos de los Insectos encontrados en Cataluña.

8. Barcelona, 1879.

MARTORELL Y PEÑA (M.). See Cuní y Martoreli (M.) \& Martorell y Peña (M.). 
MARTYN (T.). The Universal Conchologist.

4 vols. fol. London, 1784 .

MARTYN (T.). Thirty-eight plates, with explanations, intended to illustrate Linnæus's system of Vegetables.

$8^{\circ}$. London, 1788.

MARTYN (T.). Entomologiste Anglois . . Insectes Coléoptères qui se trouvent en Angleterre.

$4^{\circ}$. London, 1792.

MARVELS OF NATURE, or Outlines of Creation, by the author of "The Boy's Book of Industrial Information." Illustrations by the brothers DatzieI. $8^{\circ}$. London, n. d.

MASSALIEN (F. C.). Dissertatio inauguralis 'sistens descriptionem oculorum Scombri, Thynni et Sepiæ.

$4^{\circ}$. Berolini, 1815.

MASSMANN (J. C.). Descriptio Osteologica Cranii Myrmecophagæ tetradactylæ.

$4^{\circ}$. Berolini, 1823.

MASTERS (G.). Catalogue of the described Coleoptera of Australia.

4 parts. $8^{\circ}$. Sydney, 1871-72.

MASTERS (G.). Catalogue of the described Diurnal Lepidoptera of Australia.

8. Syclney, 1873.

MATHER (H.). Account of the Discovery and Dissection of the Ostend Whale. Translated from the French.

8․ London, 1831.

MATON (G.). The Natural History of the County of Wilts.

8. London, 1843.

MATSUBARA (S.). See Fisheries : Berlin. Internationale Fischerei-Ausstellung, 1880.

MATTEUCCI (C.) \& SAVI (P.). Traité des Phénomènes Electrophysiologiques des Animaux; suivi d'Etudes sur le Système nerveux de la Torpille, par P PUL SAvI.

8o. Paris, 1844. 
MATTHEWS (A.). Trichopterygia. $\quad 4^{\circ}$. London, 1872.

MATTHEWS (A.) \& FOWLER (W. W.). Catalogue of British Coleoptera. 8.. London, 1883.

MATZEK (C. A. E.). Necrophororum monographiæ, particula prima.

8. Vratislavice, 1839.

MAUDUYT (R. J. E.). Encyclopédie Méthodique: Ornithologie.

Tom. I. \& II. 4º Paris, 1784.

MAUMONT (J. E. Rouques de). Némoire sur les Polypiers de Mer.

8.. Zelle, 1782.

MAUPIED (F. L. M.). Considérations sur la Série Animale, ses Groupes et ses Espèces.

$4^{\circ}$. Paris, 1841.

MAWE (J.). The Linnæan System of Conchology, describing the Orders, Genera, and Species of Shells.

8. . London, 1823.

MAWE (J.). See Wodarch (C.).

MAXIMILIAN (Prinz zu Wied-Neuwied). See WIEdNEUWIED.

MAYER (A.F. J.C.). Analecten für vergleichende Anatomie.

2 parts. $4^{\circ}$. Bonn, 1835-39.

MAYER (A. F. J. C.). Spicilegium observationum anatomicarum de Organo electrico in Raiis analectricis et de Hæmatozois.

$4^{\circ}$. Bonnce, 1843.

MAYER (A. F. J. C.). System des Thier-Reiches.

8.. Bonn, 1849.

MAYER (P.). See Fauna und Flora des Golfes von Neapel.

MAYER (S.). See HeRnrann (J.). Handbuch der Physiologie. 
MAYNARD (C. J.). The Birds of Elorida.

Continued as:

Parts 1 \& 2. $4^{\circ}$. Salem, 1872-73.

Part 3. Ipswich, Mass., 1874.

The Birds of Florida with the Water and Game Birds of Eastern North America. Parts 4-9. Newtonville, 1878.

Continued as:

The Birds of Eastern North America.

Parts 10-16. Newtonville, 1879-81.

MAYO (Earl of). See JoHrston (H. H.).

MAYR (G.). Die Europäischen Cynipiden-Gallen mit Ausschluss der auf Eichen vorkommenden Arten.

8. Wien, 1876.

MAYR (G.). Die Genera der gallenbewohnenden Cynipiden.

$8^{\circ}$. Wien, 1881.

MAIR (G.). Die Europäischen Arten der gallenbewohnenden Cynipiden.

$8^{\circ}$. Wien, 1882.

MAYR (G. L.). Die Europäischen Formiciden (Ameisen). 8०. Wien, 1861.

MAYR (G. L.). See ' Norara.'

MEAD (T. L.). See Surveys \& Explorations: U.S., WASHINGTON.

MECKEL (J. F.). System der vergleichenden Anatomie. 6 vols. in 7. $4^{\circ}$. Halle, 1821-33.

MECKEL (J. F.). Ornithorhynchi paradoxi Descriptio Anatomica.

fol. Lipsice, 1826.

MECKEL(J. F.). Additamenta ad historiam Molluscorum, Piscium et Amphibiorum. 4\%. Halce, 1832.

MECKEl (J. F.). See Periodicals : Halle.

MEGERLE VON MÜHLFELD (J. K.). Abhandlung ueber die nachtheiligen Thiere. $8^{\circ}$. Wien, 1805. 
MEGERLE VON MÜHLFELD (J. K.). Bermerkungen zu Illiger's Zusätzen zu Fabricii Systema Eleutheratorum.

$12^{\circ}$. Linz, 1812.

MÉGNIN (P.). Les Parasites et les Maladies Parasitaires. Texte et Atlas. $8^{\circ}$. Paris, 1880.

MEIDINGER (C. L. B. a). Icones Piscium Austriæ Indigenorum.

fol. Viennoe, 1785.

MEIGEN (J. W.). Europäische zweiflügelige Insekten. 8 vols. $8^{\circ}$. Huchen, 1818-38.

MEIGEN (J. W.). Systematische Beschreibung der Europäischen Schmetterlinge. $\quad 4^{\circ}$. Aachen \& Leipzig, 1827-32.

MEIGEN (W.). De Marsupialium Dispositione Systematica. Dissertatio zoologica. $\quad 8^{\circ}$. Bonnce, 1851.

MEIGEN (W.). See LoEw (H.).

MEIGS (J. A.). Catalogue of Human Crania in the Collection of the Academy of Natural Sciences of Philadelphia. 8. Philadelphia, 1857.

MEINERT (F.). Fluernes Munddele, Trophi Dipterorum. 4. Kjöbenhavn, 1881.

MEINERT (F.). Caput Scolopendræ: Head of the Scolopendra and its muscular system. $4^{\circ}$. Copenhagen, 1883.

MEISNER (C. F.). De Amphibiorum quorundam papillis glandulisque femoralibus.

$4^{\circ}$. Basilece, 1832.

MEISNER (F.) \& SCHINZ (H. R.). Die Vögel der Schweiz. 8. Zurich, 1815.

MELA (A. J.). Vertebrata Fennica.

$8^{\circ}$. Helsingissa, 1882.

MELCHIOR (H. B.). Den danske Stats og Norges Pattedyr.

8. Kiobenhavn, 1834.

MELDOLA (R.). See Weismann (A.). 
MELSHEIMER (F. E.). Catalogue of the described Coleoptera of the United States. $\quad 8^{\circ}$. Washington, 1853.

MENEGAZZI (L.). Malacologia Veronese.

8.. Verona, 1855.

MENEGHINI (G.). Osservazioni Postume di Zoologia Adriatica del Professore S. A. Renrer. fol. Venezia, 1847.

MÉNÉTRIES (E.). Catalogue raisonné des Objets de Zoologie recueillis dans un Voyage au Caucase. $4^{\circ}$. St. Petersburg, 1832.

MÉNÉTRIES (E.). Monographie de la Famille des Myiotherince. 40. St. Petersburg, 1835.

MÉNÉTRIES (E.). Catalogue d'Insectes recueillis entre Constantinople et le Balkan. $\quad 4^{\circ}$. St. Petersburg, 1838.

MÉNÉTRIES (E.). Essai d'une Monographie du genre Anacolus. $4^{\circ}$. St. Petersburg, 1839.

MÉNÉTRIES (E.). Description des Insectes recueillis par feu Lehmann. $4^{\circ}$. St. Petersburg, 1848.

MÉNÉTRIES (Ev). Catalogue de la collection Entomologique de l'Académie impériale des Sciences de St. Pétersbourg. Lépidoptères. 3 parts. $8^{\circ}$. Petropoti, 1855-63.

MENETRIES (E.). See Strvers \& Explorations: Russia.

MENKE (C. T.). Synopsis methodica Molluscorum. 8. Prymonti, 1828.

MENKE (C. T.). Molluscorum Novæ Hollandiæ Specimen. 4.. Hanoverce, 1843.

MENKE (C. T.) \& PFEIFFER (L.). See Periodicals: Cassei.

MENONVILLE (Thiery de). Traité de la Culture du Nopal et de l'éducation de la Cochenille, dans les Colonies Françaises et de l'Amérique. Précédé d'un Voyage à Guaxaca. 2 vols. $8^{\circ}$. Paris \& Bordeanx, 1787. 
MEREJKOWSKY (C.). Études sur les Éponges de la Mer Blanche. $4^{\circ}$. St. Petersburg, 1878.

IIERLAN (M. S.). Der Rupsen begin, roedsel en wonderbaare rerandering. 1 rol. sm. $4^{\circ}$. Amsterdam, 1678. German translation. 2 rols. sm. $4^{\circ}$. Niimberg, 1679-83.

MERIAN (M. S.). Erucarum ortus, alimentum et paradoxa metamorphosis. $4^{0}$. Amsteldami, 1717.

MERIAN (M. S.). Orer de roortteeling en Tonderbaerlyke reranderingen der Surinaemsche Insecten.

fol. Amsterdam, 1730.

MERIAN (M. S.). De Europäische Insecten.

fol. Amsterdam, 1730 .

MERKEL (F.). Ueber die Endigungen der sensiblen Nerren in der Haut der Wirbelthiere.

$4^{0}$. Rostock, 1880.

MERKEL (F.). Beitrage zur Kenntniss der Postembryonalen Entwicklung des menschlichen Schädels.

$4^{\circ}$. Bonn, 1882 .

MERKEL (F.). Die Speichelröhren. $\quad$ \&o . Leipzig, 1883.

MERREM (B.). De Animalibus Seythicis apud Plinium.

$4^{\circ}$. Göttingce, 1781 .

IIERREM (B.). Termischte Abhandlungen aus der Thiergeschichte.

t. Göttingen, 1781 .

MERREM (B.). Versuch eines Systems der Amphibien.

8. Marburg, 1820.

MERREM (B.). Beiträge zur Naturgeschichte der Amphibien. $4^{0}$. Essen, 1821.

MERRIFIELD (Mrs.). A Sketch of the Natural History of Brighton.

$8^{\circ}$. Brighton, 1864.

MERTENS (H.). Beschreibung der Oitopleura, einer meuen Molluskengattung. $\quad 4^{\circ}$. St. Petersburg, 1803. 
MERTENS (H.). Untersuchungen über den innern Bau verschiedener in der See lebender Planarien.

$4^{\circ}$. St. Petersburg, 1832.

MERTENS (H.). Die Beroëartigen Akalephen.

$4^{\circ}$. St. Petersburg, 1833.

METAXA (L.). Monografia de' Serpenti di Roma e suoi contorni. $4^{\circ}$. Roma, 1823.

METSCHNIKOFF (E.). Studien über die Entwickelung der Echinodermen und Nemertinen.

$4^{\circ}$. St. Petersburg, 1869.

METTENHEIMER (C.). Disquisitiones anatomico-comparativæ de membro piscium pectorali. $4^{\circ}$. Berolini, 1847.

METZGER (A.). See Fisheries: Berlin. Internationalo Fischerei-A usstellung, 1880.

MEYER (A. B.). Index zu L. Reichenbach's Ornithologischen Werken. roy. $8^{\circ}$. Berlin, 1879.

MEYER (A. B.). Abbildungen von Vögel-Skeletten.

Parts I.-V. $4^{\circ}$. Diesclen, 1879-83.

MEYER (B.). Kurze Beschreibung der Vögel Liv- und Esthlands. 8. Nürnberg, 1815.

MEYER (B.) \& WOLF (J.). Taschenbuch der Deutschen Vögelkunde. 2 rols. 8०. Frankfurt am Main, 1810.

MEYER (E.). Die Spermatogenese bei den Säugethieren. $4^{\circ}$. St. Petersburg, 1880.

MEYER (F.). Ein Fall von Echinococcus multiocularis. 8․ Göttingen, 1881.

MEYER (F. A. A.). Systematisch-summarische Uebersicht der neuesten zoologischen Entdeckungen in Neuholland und Afrika.

8. Leipzig, 1793.

MEYER (H. von). Palæologica zur Geschichte der Erde und ihrer Geschöpfe. $\quad$ 8. Frankfurt am Main, 1832. 
MEYER (H. A.) \& MÖBIUS (K.). Fauna der Kieler Bucht. Die Prosobranchia und Lamellibranchia.

4. Leipzig, 1872.

MEYER (H. A.), MÖ̈BIUS (K.), KARSTEN (G.), \& HENSEN (V.). Gemeinfassliche Mittheilungen aus den Untersuchungen der Kommission zur wissenschaftlichen Untersuchung der deutschen Meere. $\quad 8^{\circ}$. Kiel, 1880.

MEYER (H. L.). Coloured Illustrations of British Birds, and their Eggs. 7 vols. $8^{\circ}$. London, 1842-50. Another edition. 4 vols. $4^{\circ}$. London [1852].

MEYER (H. L.). Game Birds and their Localities.

obl. $4^{\circ}$. London, 1848.

MEYER (J. B.). Aristotelis Thierkunde.

See Aristoteles.

$8^{\circ}$. Berlin, 1855.

MEYER (J. D.). Angenehmer Zeitrertreib mit Betrachtung curioser Vorstellungen allerhand Thiere als auch ihrer Scelete.

fol. Nürnberg, 1748.

ILEYER (J. D.). Vorstellungen allerhand Thiere, mit ihren Gerippen. $\quad 2$ vols. fol. Nürnberg, 1748-52.

MEYER (J. R. E. v.). Beyträge zur Anatomie des Tiegers, nebst allgemeinen Bemerkungen über die Katzengattung.

$8^{\circ}$. Wien, 1826.

MEYER (L. R.). Verzeichniss der in der Schweiz einheimischen Rhynchoten (Hemiptera, Linn.).

8․ Solothurn, 1843.

MEYER (N.). Prodromus Anatomiæ Murium.

$4^{\circ}$. Jence, 1800.

MEYER (P.). Etudes histologiques sụr le Labyrinthe membraneux et sur le limaçon [cochlea] chez les Reptiles et les Oiseaux.

8․ Strasbourg, 1876.

MEYER-DÜR (L. R.). Verzeichniss der Schmetterlinge der Schweiz. Erste Abtheilung, Tagfalter.

$4^{\circ}$. Ziïich, 1851. 
MICHAELIS (K.). See Borne (Von dem).

IIICHAUD (A. L. G.). Complément des Mollusques de la France. $4^{\circ}$. Verdun, 1831.

MICHAUD (A. L. G.). See Potiez (V. L. V.) \& Michadd (A. L. G.).

MICHEL ( ). Les Perroquets.

12. Paris, 1829.

MIDDENDORFF (A. T. von). Einblikke in das FerghanaThal. $4^{\circ}$. St. Petersburg, 1881.

MIDDENDORFF (A. T. von). Beiträge zu einer Malacozoologia Rossica. 4․ St. Petersburg, 1847.

MIDDENDORFF (A. T. ron). Reise in den äussersten Norden und Osten Sibiriens.

$4^{\circ}$. St. Petersburg, 1855-75. See Surveys \& Explorations: Russia.

MIDDENDORFF (A. T. v.). Die Isepiptesen Russlands. Grundlagen zur Erforschung der Zugzeiten und Zugrichtungen der Vögel Russlands. $\quad 4^{\circ}$. St. Petersburg, 1855.

MIDDENDORFF (А. T. จ.). Die Barabá. $4^{\circ}$. St. Petersburg, 1870.

MIERS (E. J.). Catalogue of the Stalk-eyed and Sessileeyed Crustacea of New Zealand.

8.. London, 1876.

MIERS (E. J.). See 'Erebus' and 'Terror.'

MIHALKOVICS (V.). Entwickelungsgeschichte des Gehirns.

$4^{\circ}$. Leipzig, 1877.

MIK (J.). Dipterologische Untersuchungen.

$8^{\circ}$. Wien, 1878.

MIKAN (J. C.). Entomologische Beobachtungen, Berichtigungen und Entdeckungen. $\quad 4^{\circ}$. Prag, 1797. 
MIKLUCHO-MACLAY (N. von). Beiträge zur vergleichenden Neurologie der Wirbelthiere.

$4^{\circ}$. Leipzig, 1870.

MIKLUCHO-MACLAY (N. von). Ueber einige Schwämme des nördlichen stillen Oceans und des Eismeeres.

4\%. St. Petersburg, 1870.

MILLER (Dr.). Die Schalthiere des Bodensee's.

$8^{\circ}$. Lindau, 1873.

MILLER (J. S.). A Natural History of the Crinoidea. $4^{\circ}$. Bristol, 1821.

MilleR, (L.). See Perrodicals: Vienna.

MILLIERE (P.). Iconographie et Description de Chenilles et Lépidoptères inédits.

3 vols. $8^{\circ}$. Paris, 1859-69.

MILLIES (H. C.). Over eene niew ontdekte Afbeelding van den Dodo. $4^{\circ}$. Amsterdam, 1868.

MILNE-EDWARDS (A.). Faune ornithologique éteinte des îles Mascareignes et de Madagascar.

4. Paris, 1866-73.

MILNE-EDWARDS (A.). Recherches pour servir à l'histoire naturelle des Mammifères.

2 vols. $4^{\circ}$. Paris, $1868-74$.

MILNE-EDWARDS (A.) \& GRANDIDIER (A.). See Grandidier (A.).

MILNE-EDWARDS (A.). See Surveys \& Explorations : France.

MILNE-EDWARDS (H.). Éléments de Zoologie.

Text 1 vol. \& Atlas. roy. $8^{\circ}$. Bruxelles, 1837. Another edition. $8^{\circ}$. Paris, 1843.

MILNE-EDWARDS (H.). Histoire naturelle des Crustacés.

8․ Paris, 1834-40. 
MILNE-EDWARDS (H.). Histoire naturelle des Coralliaires ou Polypes.

3 vols. and Atlas. $8^{\circ}$. Paris, 1857-60.

MILNE-EDWARDS (H.). Rapport sur les progrès récents des Sciences Zoologiques en France.

8.. Paris, 1867.

MILNE-EDWARDS (H.). Leçons sur la Physiologie et l'Anatomie comparée. 14 vols. 8०. Paris, 1876-81.

MILNE-EDWARDS (H.). Cours élémentaire d'Histoiro Naturelle. $\quad 12$ th edition. post $8^{\circ}$. Paris, 1877.

MILNE-EDWaRdS (H.). See Periodicals: Paris.

MILNE-EDWARDS (H.), BLANCHARD (E.), \& LUCAS (H.). Catalogue de la collection entomologique du Muséum d'histoire naturelle de Paris. Coléoptères.

See Museum (Paris).

8. $\quad$ Paris, 1850.

MILNE-EDWARDS (H.) \& HAIME (J.). A Monograph of the British Fossil Corals.

4.. London, 1850.

MILNE-EDWARDS (H.) \& HAIME (J.). Monographio des Polypiers fossiles.

$4^{\circ}$. Paris, 1851.

MILNE-EDWARDS (H.), QUATREFAGES (A. de), \& BLANCHARD (E.). Recherches anatomiques et zoologiques.

$4^{\circ}$. Paris, 1844.

MINDING (J.). Ueber die geographische Vertheilung der Säugethiere.

$4^{\circ}$. Berlin, 1829.

MITCHELL (J. M.). The Herring. $8^{\circ}$. Edinburgh, 1864.

MITTERPACHER (L.). See Piller (M.).

MIVART (St. G.). On the Genesis of Species. 2nd edition. $8^{\circ}$. London, 1871.

MIVART (St. G.). Man and Apes. $\quad 8^{\circ} . \quad$ London, 1873. 
MIVART (St. G.). The Common Frog.

8. London, 1874.

MIVART (St. G.). The Cat.

8. London, 1881.

MÖBIUS (K.). Neue Gorgoniden des naturhistorischen Museums zu Hamburg. $4^{\circ}$. Jena, 1861.

MÖBIUS (K.). Uobor Austern und Miesmuschelzucht. 8. Berlin, 1870.

MÖBIUS (K.). Die Bewegungen der fliegenden Fische.

8. Leipzig, 1878.

MÖBIUS (K.). See Merer (H. A.) and others.

MÖBIUS (K.), RICHTER (F.), \& MARTENS (E. von). Beiträge zur Meeresfauna der Insel Mauritius und der Seychellen. $4^{\circ}$. Berlin, 1880.

MOCQUERYS (E.). Recueil de Coléoptères anormaux.

8. Rouen, 1853-65.

MOCSÁRY (S.). Magyar Fauna Fémdarázsai Chrysididæ Faunæ Hungaricæ. $4^{\circ}$. Budapest, 1882.

MODEER (A.). Bibliotheca Helminthologica.

8. Erlangce, 1786.

MOEHRING (P. H. G.). Avium genera.

8. Bremce, 1752.

MOGGRIDGE (J. T.). Harvesting Ants and Trap-door Spiders. 2 parts. $8^{\circ}$. London, 1873-74.

MOHR (N.). Forsog til en Islandsk Naturhistorie.

8. Kjöbenhavn, 1786.

MOHRENSTERN (G. S. v.). Über die Familie der Rissoiden.

$4^{\circ}$. Wien, 1860.

MOHRING (C. A.). Dissertatio sistens descriptionem Trionychos Agyptiaci osteologicam. $4^{\circ}$. Berolini, 1824. 
MOJSVĀR (A. M. Edlen ron). Leitfaden bei zoologischzootomischen Präparirübungen für Studirende.

8. Leipzig, 1879.

MOLLENDORFF (0. จ.). Beiträge zur Fauna Bosniens.

8․ Görlitz, 1873.

MÖLLER (G.). Skandinaviens Skalbaggar (Coleoptera).

Part I. sm. $4^{\circ}$. Lund, 1862.

IÖLLER (G.). Kort Beskrifning öfrer Skandinariens Skalbaggar (Coleoptera). 2 parts. $8^{\circ}$. Lund, 1863-66.

MÖLLER (H. P. C.). Index Molluscorum Grœnlandiæ.

8. Hafnice, 1842.

MÖLLER (J. O. L.). Observata quædam anatomica de Auchenia lama.

8. Regimontii, 1840.

MÖLLER (L.). Die Abhängigkeit der Insecten von ihrer

Umgebung.

8. Leipzig, 1867.

MÖLLER (V. г.). Die spiral-gewundenen Foraminiferen des russischen Kohlenkalks. $\quad 4^{\circ}$. St. Petersburg, 1878.

MÖLLER (V. г.). Die Foraminiferen des russischen Kohlenkalks. $\quad 4^{\circ}$. St. Petersburg, 1879.

MOLONEY (Capt.). See Fisheries: London. International Fisheries Exhibition, 1883.

MONCEAU (H.). See Robiveat-Destoidt (Dr.).

MONIEZ(R.). Essai monographique sur les Cysticerques. $4^{\circ}$. Paris, 1880.

MONIEZ (R.). Mrémoires sur les Cestodes. Première partie. 4. Paris, 1881.

MONRO (A.). Vergleichung des Baues und der Physiologie der Fische. (Translated from English by J. G. Sinneider.) 2 rols. $4^{\circ}$. and Atlas. Leipzig, 1787. 
MONTAGU (G.). Ornithological Dictionary.

2 vols. and Suppl. 80. London, 1802-13. Another edition by J. Revnie. $\quad 8^{\circ}$. London, 1831 . Another edition by E. Newman. $8^{\circ}$. London, 1866.

MONTAGU (G.). Testacea Britannica.

3 vols. $4^{\circ}$. London, 1803.

Monterosato (M. di). Catalogo dello Conchiglie Mediterranee. $4^{\circ}$. Putermo.

MONTEROSATO (M. di). Notizie intorno ai Solarii del Mediterraneo.

8․ Patermo, 1873.

MONTI (M.). Catalogo e notizie compendiose degli Uccelli, di stazione e di passaggio nella città, provincia e diocesi di Como e loro Comasca sinonimia. $16^{\circ}$. Como, $18 \pm 5$.

MONTROUZIER (Le P.). Essai sur la Faune de l'Ile de Woodlark. $8^{\circ}$. Lyon, 1857.

MOOR (B. de). De Piscium et Avium Creatione.

4. Harderovici, 1716.

MOORE (F.). The Lepidoptera of Ceylon.

Parts I.-VII. 4\%. London, 1880-83.

MOORE (F.). See Horsfield (T.).

MOORE (F.). See Hewitson (W. C.), Moore (F.), \& Grote (A.).

MOORE (F.). See Survers \& Explorations: Yarkand.

MOORE (J.). Columbarium, or the Pigeon House: an introduction to a Natural History of tame Pigeons. 1735.

(Reprint by W. B. Tegetmeier.) $8^{\circ}$. London, 1879.

MOORE (T. J.). Dimensions of a large Gorilla in the Liverpool Free Public Museum. $\quad 8^{\circ}$. Liverpool, 1862.

MOQUIN-TANDON (A.). Monngraphie de la Famille des Hirudinées. Text and Atlas. $8^{\circ}$. Paris, 1846 . 
MOQUIN-TANDON (A.). Histoire naturelle des Mollusques de France. 2 vols. and Atlas. 80. Paris, 1855.

MORANVILLE (L. S. von). Die Vögel Europa's.

16. Wien, 1844.

MORAWITZ (A.). Beitrag zur Käferfauna der Insel Jesso. Cicindelida et Carabici.

$4^{\circ}$. St. Petersburg, 1863.

MÖRCH (O. A. L.). Catalogus Conchyliorum quæ reliquit C. P. Kierulf.

8.. Hafnice, 1850.

MÖRCH (O. A. L.). Catalogus Conchyliorum quæ reliquit d'Aguirra. 8. Hafnice, 1852-3.

MÖRCH (O. A. L.). Prodromus Faunæ Molluscorum Grönlandiæ. $\quad$ 80. Kjöbenhavn, 1857.

MÖRCH (O. A. L.). Synopsis Molluscorum terrestrium et fluviatilium Daniæ. $\quad 8^{\circ}$. Kjöbenhavn, 1864.

MöRCH (O. A. L.). A Descriptive Catalogue of the Scalidee of the West India Islands.

4. Philadelphia, 1876.

MORE (A. G.). See Brown (J. A. H.) and others.

MOREAU (E.). Histoire Naturelle des Poissons de la France. 3 vols. $8^{\circ}$. Paris, 1881.

MORELET (A.). Description des Mollusques terrestres et fluviatiles du Portugal. $\quad$ 80. Paris, 1845.

MORELET (A.). Testacea Novissima Insulæ Cubanæ et Americæ Centralis. Parts $1 \& 2$. 80. Paris, 1849-51.

MORELET (A.). Séries Conchyliologiques.

Parts I.-IV. 80. Paris, 1858-75.

MORELET(A.). Notice sur l'histoire naturelle des Açores. 8. Paris, 1860.

MORELET (A.). See Welwitsch (F.). 
MORREN (C. F. A.). De Historiâ Naturali Lumbrici Terrestris. 4\%. Gandavi, 1826.

MORRIS (B. R.): British Game Birds and-Wildfowl.

4. London, 1855.

MORRIS (F. O.). A History of British Birds.

8 vols. $8^{\circ}$. London, 1851-70. 2nd edition. 6 vols. post $8^{\circ}$. London, 1870.

MORRIS (F. O.). A Natural History of the Nests and Eggs of British Birds. 3 vols. $8^{\circ}$. London, 1861-63.

MORRIS (F. O.). A Natural History of British Moths.

4 vols. $8^{\circ}$. London, 1861-70.

MORRIS (F. O.). A History of British Butterflies. 4th edition. 1 vol. $8^{\circ}$. London, 1876.

MORRIS (J.). See Museum: College of Surgeons.

MORRIS (J. G.). Catalogue of the described Lepidoptera of North America.

8. Washington, 1860.

MORRIS (J. G.). Synopsis of the described Lepidoptera of North America. $\quad$ Part I. $8^{\circ}$. Washington, 1862.

MORSE (E. S.). A Classification of Mollusca, based on the principle of Cephalization.

MORSE (E. S.). Embryology of Terebratulina.

$4^{\circ}$. Boston, 1873.

MORTIMER (C.). Some Papers lately read before the Royal Society concerning the Fresh-water Polypus.

$4^{\circ}$. London, 1743.

MORTON (S. G.). Crania Americana.

fol. Philadelphia, 1839.

MORTON (S. G.). Crania Egyptiaca.

$4^{\circ} . \quad$ London, 1844.

MORTON (S. G.). Catalogue of Skulls of Man and the Inferior Animals in the collection of S. G. Morton.

3rd edition. $8^{\circ}$. Philadelphia, 1849. 
MORTON (S. G.). Notes on Hybridity.

8. Philadelphia, 1850.

MÖSCHLER (H. B.). Die Familien und Gattungen der Europäischen Tagfalter.

8. Görlitz [1880?].

MÖSCHLER (H. B.). Beiträge zur Schmetterlings-Fauna von Surinam. Parts I.-IV. $8^{\circ}$. Wien, 1881.

MOSCROP (E. H.). Correspondence on the Introduction of Salmon and Trout at the Antipodes. $8^{\circ}$. London, 1879.

MOSELEY (H. N.). Notes by a Naturalist on the 'Challenge:.' 8. London, 1879.

MOSELEY (H. N.). See 'Challenger.'

MOSENTHAL (J. de) \& HARTING (J. E.). Ostriches and Ostrich Farming. 2nd edition. $8^{\circ}$. London, 1879.

MOSLEY (S. L.). Illustrations of Varieties of British Iepidoptera.

Parts I.-XII. roy. 8. Huddersfield, 1878-83.

MOTLEY (J.) \& DILLWYN (L. L.). Contributions to the Natural History of Labuan. 8. London, 1855.

MOTSCHULSKY (V. de). Insectes de la Sibérie.

$4^{\circ}$. St. Petersburg, 1844.

MOTSCHULSKY (V. จ.). Die Coleopterologischen Verhältnisse und die Käfer Russlands. $\quad 8^{\circ}$. Moscau, 1846.

MOTSCHULSKY (V. de). Études Entomologiques.

8. Helsingfors, 1852-62.

MOUFET (T.). Insectorum sive Minimorum Animalium Theatrum. $4^{\circ}$. London, 1634.

MOUSSON (A.). Die Land- und Süsswasser-Mollusken von Java.

8.. Zürich, 1849.

MOUSSON (A.). Coquilles terrestres et fluviatiles recueillies dans l'orient par M. le Dr. A. Schla efrir.

8.. Ziirich, 1859. 
MOUSSON (A.). Coquilles terrestres et fluviatiles recueillies par M. le prof. J. R. Rotr dans son dernier voyage en Palestine.

8. Zürich, 1861.

MOUTON-FONTENILLE (J. P.). Observations sur la Marmotte. 8. Lyon, 1808.

MUFFETT (T.). See Modfet (T.).

MÜHLE (H. G. von der). Beiträge zur Ornithologie Griechenlands. 8. Leipzig, 1844.

MUHR (J.). Ueber die Mundtheile der Orthoptera.

8. Prag, 1877.

MUIR (J.). See 'CoRwIN.'

MÜLLER (A.). Ueber die erste Entstehung organischer Wesen und deren Spaltung in Arten. $\quad 8^{\circ}$. Berlin, 1881.

MÜLLER (A. \& K.). Thiere der Heimath. 2 vols. roy. $8^{\circ}$. Kassel \& Berlin, 1882.

MÜLLER (F.). Für Darwin. 8. Leipzig, 1864.

MÜLLER (J.). De Phoronomia Animalium. $4^{\circ}$. Bonnce, 1822.

MÜLLER (J.). Handbuch der Physiologie des Menschen. 2 vols. $8^{\circ}$. Coblenz, 1838-40.

MÜLLER (J.). On the Structure and Characters of tho Ganoidei, and on the natural Classification of Fish. Translated by J. W. Griffith, M.D. 8. Berlin, 1844.

MÜLLER (J.). Ueber den Bau und die Grenzen der Ganoiden, und über das natürliche System der Fische.

fol. Berlin, 1846.

MÜLLER (J.). Ueber die Larven und die Metamorphose der Ophiuren und Seeigel.

$4^{\circ}$. Berlin, 1848.

MÜLLER (J.). Ueber die Larven und die Metamorphose der Holothurien und Asterien.

4. Berlin, 1850. 
MÜLLER (J.). Ueber die Larven und die Metamorphose der Echinodermen.

$4^{\circ}$. Berlin, 1852.

MÜLLER (J.). Ueber Synapta digitatce und über die Erzeugung von Schnecken in Holothurien.

$4^{\circ}$. Berlin, 1852.

MÜlleR (J.). See Periodicals: Berlin, Halie, \& Leipzia.

MÜLLER (J.) \& HENLE (J.). Systematische Beschreibung der Plagiostomen. fol. Bertin, 1838-41.

MÜLLER (J.) \& TROSCHEL (F. H.). System der Asteriden.

$4^{\circ}$. Braunschweig, 1842.

MÜLLER (J.) \& TROSCHEL (F. H.). Horæ Ichthyologicæ.

$4^{\circ}$. Berlin, 1845-49.

MÜLLER (J.). Terminologia Entomologica.

8.. Brïnn, 1860.

MÜLLER (J. W. de). Description de Noureaux Oiseaux d'Afrique. 5 parts. $4^{\circ}$. Stuttgart, 1853-54.

MÜLLER (O. F.). Fauna Insectorum Fridrichsdalina.

8.. Hafnice \& Lipsice, 1764.

MÜLLER (O. F.). Von Würmern des süssen und salzigen Wassers. $4^{\circ}$. Kopenhagen, 1771.

MÜLLER (O. F.). Termium terrestrium et fluviatilium, seu Animalium Infusoriorum, Helminthicorum et Testaceorum, non marinorum, succincta historia.

2 vols. $4^{\circ}$. Havnice \& Lipsice, 1773-74.

MÜLLER (O. F.). Zoologiæ Danicæ Prodromus.

8. Haunice, 1776.

MÜLLER (O. F.). Zoologia Danica.

fol. Hafnice, 1788-1806. Another edition. $8^{\circ}$. Havnice \& Lipsice, 1779-84. 
MÜLLER (O. F.). Hydrachnæ quas in aquis Daniæ palustribus detexit O. F. MÜLLER. $\quad 4^{\circ}$. Lipsice, 1781.

MÜLLER (O. F.). Entomostraca seu Insecta Testacea. 4. Lipsice \& Havnice, 1785.

MÜ̈LLER (O. F.). Animalcula Infusoria. Ed. O. FABRIcros.

$4^{\circ}$. Havnice, 1786.

MÜLLER (O. F.). Naturgeschichte einiger Wurm-Arten des süssen und saltzigen Wassers. $4^{\circ}$. Kopenhagen, 1800.

MÜLLER (P. E.). Danmarks Cladocera.

8․ Kjöbenhavn, 1867.

MÜLLER (P. E.). Iagttagelser over Nogle Siphonophorer. 8. Kjöbenhavn, 1871.

MÜLLER (P. W. J.). See IrseKteNgeschichte.

MÜLLER (S.). See Temrarck (C. J.). Verhandelingen.

HÜLLER (Th.). Synopsis Testaceorum viventium.

8. Berolini, 1836.

MULSANT (E.). Lettres à Julie sur l'Ornithologie. $8^{\circ}$. Paris, 1830.

MULSANT (E.). Lettres à Julio sur l'Entomologie.

2 vols. $8^{\circ}$. Lyon \& Paris, 1830.

MULSANT (E.). Histoire naturelle des Coléoptères de France.

10 vols. $8^{\circ}$. Paris, 1839-68.

MIULSANT (E.). Species des Coléoptères trimères sécuripalpes.

8. Lyon, 1851.

Another copy.

MULSANT (E.). Opuscules Entomologiques.

8. Paris, 1852-75.

MULSANT (E.). Histoire Naturelle des Punaises de France.

Parts I.-IV. $8^{\circ}$. Paris, 1865-73. 
MULSANT (E.) \& VERREAUX (J. \& E.). Essai d'une classification méthodique des Trochilidés ou OiseauxMouches.

8. Paris, 1865.

MULSANT (E.) \& VERREAUX (E.). Histoire Naturelle des Oiseaux-MLouches ou Colibris (Trochilidés).

4 vols. $4^{\circ}$. Lyon, 1874-77.

MUNDAHL (C. H.). See Frsmeries : London. International Fisheries Exhibition, 1883.

MURRAY (A.). Catalogue of the Coleoptera of Scotland. 8. Edinburgh \& London, 1853.

MURRAY (A.). The Geographical Distribution of Mammals. $4^{\circ}$. London, 1866.

MURRAY (A.). Economic Entomology. Aptera.

8. London, 1877.

MURRAY (J.). Experimental Researches.

8.. Glasgow, 1826.

MURRAY (J.). The Natural History. of the Silk-Worm. 2nd edition. $8^{\circ}$. London, 1838.

MUSEUM (AMSTERDAM). Seo Oltmans (A.).

MUSEUM (AUSTRALIAN).

Catalogue of Natural History in the Australian Museum. By G. Bennetr. $\quad 8^{\circ}$. Sydney, 1837.

Catalogue of the Specimens of Natural History in the Australian Museum, Sydney, N. S. Wales. By G. Bennetr. $\quad 8^{\circ}$. Sydney, 1837.

Catalogue of the Mammalia. By G. KrefFt. 16. Sydney, 1861.

Catalogue of the Minerals and Rocks in the Australian Museum, Sydney, N. S. Wales. By G. Krefit.

8. Sydney, 1873.

Catalogue of the Australian Stalk- and Sessile-eyed Crustacea. By W. A. HASWELL.

8. Sydney, 1882. 
MUSEUM (BERLIN). See Lichtenstein (H.).

MUSEUM (BRESLAU). See Gravenhorst (J. L. C.).

MUSEUMI (BRITISH).

Mammalia.

List of Mammalia. By J. E. Gray. $\quad 12^{\circ}$. 1843.

Catalogue of Mammalia.

Part I. Cetacea. By J. E. Gray. 120. 1850.

Part II. Pinnipedia. By J. E. Gray. 120. 1850.

Part III. Ungulata. By J.E. Gray. 12 1852.

Catalogue of Carnivora, Pachydermata, and Edentata. By J. E. GraY. $\quad 8^{\circ} .1869$.

Catalogue of Ruminantic (Pecora, Linn.). By J. E. Gray.

$8^{\circ}$. 1872.

Hand-list of Edentata, Pachydermata, and Ruminantia. By J. E. Gray.

$8^{\circ} .1873$.

Catalogue of Seals \& Whales. By J.E. GruY. 8․ 1866. Supplement. By J. E. Gray. $\quad 8^{\circ}$. 1871.

Hand-list of Seals, Morses, Sea-lions, and Sea-bears. By J. E. Gray. $\quad$ 8. 1874.

Catalogue of Monkeys, Lemurs, and Fruit-eating Bats. By J. E. GraY.

8०. 1870 .

Catalogue of Chiroptera. By G.E. Dobson. $\quad 8^{\circ}$. 1878.

Catalogue of the Mammalia and Birds of New Guinea. By J. E. and G. R. GraY. $\quad 8^{\circ} .1859$.

Catalogue of the Specimens and Drawings of Mammals, Birds, Reptiles, and Fishes of Nepal and Thibet, presented by B. H. Hodgson to the British Museum. By J. E. and G. R. Gray. 12。 1846.

The same.

2nd edition. $12^{\circ} .1863$.

List of Osteological Specimens. ByJ.E.Gray. $12^{\circ} .1847$.

Catalogue of the Bones of the Mammalia. By E. GERRARD.

$8^{\circ} .1862$.

Aves.

List of Birds.

Part I. Accipitres. By G. R. Gray. 120. 1844. 2nd edition. $12^{\circ}$. 1848. 
Part II. Passeres (Fissirostres). By G. R. GRAy. $12^{\circ} .1844$.

Part III. Gallince, Gralloe, Anseres. By G. R. Gray. $12^{\circ}$. 1844. Ramphastidoe. By G. R. Gray. 12\% 1855. Psittacidae. By G. R. GraY. $12^{\circ} .1859$. Capitonidce and Picidce. By G. R. Gray.

$8^{\circ} .1868$.

Part IV. Columbce. By G. R. Gray. $8^{\circ} .1856$. Part V. Gallince. By G. R. Gray. $12^{\circ} .1867$.

Catalogue of the Genera and Subgenera of Birds. By G. R. Gray.

$12^{\circ}$. 1855 .

Catalogue of the Birds of the Tropical Islands of the Pacific Ocean. By G. R. Gray. $\quad 8^{\circ} .1859$.

Nomenclature of Birds. By G. R. GraY.

(Unpublished.)

Hand-list of Genera and Species of Birds. By G. R. Gray. 3 vols. $8^{\circ}$. 1869-71.

Catalogue of Birds.

Vol. I. Accipitres. By R. B. Sharpe. $\quad 8^{\circ} .1874$.

Vol. II. Striges. By R. B. SHarpe. $8^{\circ} .1875$.

Vol. III. Passeres (Coleomorphæ). By R. B. ShaRpe.

Vol. IV. Passeres (Cichlomorphæ). By R. B. Sharpe. $\quad 8^{\circ} .1879$.

Vol. V. Passeres (Cichlomorphæ). Ву Н. Аеввонм.

Vol VI Passeres (Cichlomorn) $\quad 8^{\circ} .1881$.

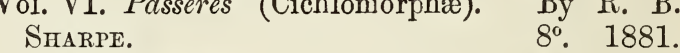

Vol. VII. Passeres (Cichlomorphæ). By R. B. Sharpe. $\quad 8^{\circ} .1883$.

Vol. VIII. Passeres (Cichlomorphæ, Certhiomorphæ). By H. GADOw. $\quad 8^{\circ} .1883$.

vol. IX. Passeres (Cynnirimorphæ). By H. GADOW. $8^{\circ}$. 1884.

Guide to the Gould Collection of Humming-Birds in the British Museum. $\quad 8^{\circ} .1881$.

The same.

2nd edition. $8^{\circ} .1883$. 
MUSEUM (BRITISH), continued.

RePtilita.

Catalogue of Snakes. [Viperida, Crotalida, Hydridae, Boidce.] By J. E. GraY.

$12^{\circ} .1849$.

Catalogue of Colubrine Snakes. By A. Günther.

$12^{\circ} .1858$.

Catalogue of Lizards. By J. E. Gray. $\quad 8^{\circ}$. 1845.

Catalogue of Tortoises, Crocodiles, and Amphisbænians. By J. E. Gray. $12^{\circ}$. 1844.

Catalogue of Shield Reptiles. By J. E. GraY.

I. Testudinata. II. Emydosaurians.

Supplement to same. Part I.

$4^{\circ} .1855,1872$.

$4^{\circ} .1870$.

Appendix to same. Part I.

$4^{\circ} .1872$.

Hand-list of Shield Reptiles. By J. E. GRaY.

$8^{\circ} .1873$.

The Gigantic Land-Tortoises (Living and Extinct) in the British Museum. By A. Günther. $4^{\circ} .1877$.

\section{Aupmibia.}

Catalogue of the Batrachia Gractientia. By J. E. GraY. $12^{\circ}$. 1850.

-. 2nd edition. By G. A. Bodlenger. $8^{\circ} .1882$.

Catalogue of the Batrachia Salientia. By A. Günther. $8^{\circ} .1858$.

-. 2nd edition. By G. A. Bodlenger. $8^{\circ}$. 1882. PIsces.

List of Fish. Part I. Chondropterygii. By J. E. Gray. $12^{\circ}$. 1851.

Catalogue of Fish collected and described by L.T. GroNow. $12^{\circ}$. 1854.

Catalogue of Apodal Fish. By J. J. KAUP. 12 . 1856.

Catalogue of Lophobranchiate Fish. By J. J. KAUP.

Catalogue of Fishes. By A. Günther.

Vols. I.-VIII. $8^{\circ}$. 1859-70.

\section{Mollusca.}

Guide to the Systematic Distribution of Mollusca. By J. E. GraY.

$8^{\circ} .1857$.

ZOOL. CAT. 
MUSEUM (BRITISH), continued.

Catalogue of Mollusca. By J. E. Gray.

Part I. Cephalopoda Antepedia. $\quad 12^{\circ} .1849$.

Part II. Pteropoda. $12^{\circ} .1850$.

Part III. Placentida \& Anomiida. 120. 1850.

Part IV. Brachiopoda Ancylopoda. 12º 1853.

List of Mollusca. By J. E. Gray.

Part I. Volutidce.

Part II. Olividae.

$12^{\circ} .1855$.

$12^{\circ} .1865$.

Catalogue of Phaneropneumona, or Terrestrial Operculated Mollusca. By L. Pfeiffer. $12^{\circ} .1852$.

Catalogue of Auriculictu, Proserpinida, and Truncatellida. By L. Pfeiffer. $12^{\circ}$. 1857.

Catalogue of Pulmonata. By J. E. Gray. 12 1855. List of Shells of the Canaries. By A. D'Orbigny.

$12^{\circ} .1854$.

List of Shells of Cuba. By A. D'Orbigny. 12․ 1854. List of Shells of South America. By A. D'Orbignr. $12^{\circ}$. 1854 .

List of Mollusca collected by Eydoux and Souleyet. $12^{\circ}$. 1855.

Catalogue of Mazatlan Shells. By P. P. Carpenter. $12^{\circ} .1857$.

Nomenclature of Molluscous Animals and Shells.

Part I. Cyclophoridce. By W. BAIRD. 120. 1850.

Catalogus Concharum Bivalvium quæ in Musæo Britannico asserrantur.

Pars I. Venerida. By G. P. Deshayes. 12․ 1853. Pars II. Petricolidoe.

$12^{\circ}$. 1854 .

Poltzoa.

Catalogue of Marine Polyzoa. By G. Busk.

Parts I.-III. Cheilostomata. 8. 1852, 1854, 1875.

ARTHROPODA.

List of Crustacea. By A. WHIтE. $12^{\circ} .1847$.

Catalogue of Crustacea. Part I. Leucosiadce. By T. BELI.

$12^{\circ}$. 1855.

Catalogue of Amphipodous Crustacea. By C. S. BAtE. 8. 1862.

List of Myriapoda. By G. Newport. 12 . 1844. 
MUSEUM (BRITISH), continued.

Catalogue of Myriapoda. Part I. Chilopoda. By G。 NEWPORT.

$12^{\circ}$. 1856 .

Nomenclature of Coleopterous Insects. Parts I.-IV. By A. White. Parts V.-VI. By F. Surth.

$12^{\circ} .1847-52$.

Catalogue of Coleopterous Insects. Parts VII.-VIII. By A. White. Part IX, By C. H. Boheman.

$12^{\circ}$. $1853-56$.

Catalogue of the Coleopterous Insects of Madeira. By T. V. Wollaston.

$8^{\circ} .1857$.

Catalogue of the Coleopterous Insects of the Canaries. By T. V. Wollaston.

$8^{\circ} .1864$.

Catalogue of Halticidce. By H. Clark.

Part I. Physapodes and Edipodes. $\quad 8^{\circ} .1860$.

Catalogue of Hispidce. Part I. By J. S. Batr.

$8^{\circ}$. 1858.

Illustrations of Typical Specimens of Coleoptera in the Collection of the British Museum. Part I. Lycida. By C. O. Waterhodse.

$8^{\circ} .1879$.

List of Hymenopterous Insects. By F. WALKER. Parts I. \& II. Chalcidites. $\quad 12^{\circ} .1846-48$.

Catalogue of Hymenopterous Insects. By. F. Sмгтн. Parts I.-VII. $122^{\circ}$. 1853-59.

List of Lepidopterous Insects. By E. Doubledar. Part I. $12^{\circ} .1844$. Part II. \& Appendix. $12^{\circ} . \quad 1847-48$.

Catalogue of Lepidopterous Insects. Part I. Papilionidce. By G. R. Gray. $\quad 4^{\circ}, 1852$.

List of the Specimens of Lepidopterous Insects. Part I. Papitionida. By G. R. Gray. 120. 1856.

List of Lepidopterous Insects. Parts I.-XXXV. By F. WALKER.

$8^{\circ}$. 1854-1865.

Catalogue of Diurnal Lepidoptera (Satyridce). By A. G. Butler.

$8^{\circ} .1868$.

Catalogue of Diurnal Lepidoptera described by Fabricius. By A. G. Butler. $\quad 8^{\circ} .1869$.

Illustrations of Typical Specimens of Lepidoptera Heterocera. Parts I.-III. \& V. By A. G. Butler. Part IV. by Lord Walsingham. $\quad 4^{\circ}$. 1877-79. 
MUSEUM (BRITISH), continued.

List of Dipterous Insects. Parts I.-VII. By F. WALKER.

$12^{\circ}$. $1848-55$.

Catalogue of Orthopterous Insects. Part. I. Phasmidce. By J. O. Westwood. $\quad 4^{\circ} .1859$.

Catalogue of Specimens of Blattarice. By F. WALKER. $8^{\circ} .1868$.

Catalogue of Specimens of Dermaptera Saltatoria, and Supplement to Blattarice. Parts I.-V. By F. Walker. $8^{\circ}$. 1869-71.

Catalogue of Neuropterous Insects. Parts I.-IV. By F. WALKER. $12^{\circ}$. 1852-53.

Catalogue of Neuropterous Insects. Part I. Termitina. By Dr. H. HAGEN. $12^{\circ} .1858$.

List of Hemipterous Insects. Parts I.-II. By W. S. DaLLAS.

$12^{\circ}$. 1851-52.

Catalogue of Heteropterous Hemiptera. Parts I.VIII. By F. WALKER. $\quad 8^{\circ}$. 1867-73.

List of Homopterous Insects. Parts I.-IV. By F. WALKER.

$8^{\circ}$. $1850-52$. Supplement. $8^{\circ} .1858$.

Echinodermata.

Catalogue of Recent Echinida. Part I. Echinida Irregularia. By J. E. GraY. $12^{\circ}$. 1855.

\section{VERMES.}

Catalogue of Entozoa, or Intestinal Worms. By W. BAIRD.

$12^{\circ}$. 1853.

Catalogue of British Non-parasitical Worms. By G. JoHNSTON.

$8^{\circ} .1865$.

Ceelenterata.

Catalogue of Lithophytes, or Stony Corals. By J. E. GraY. $8^{\circ}$. 1870.

Catalogue of Pennatulariidce or Sea-Pens. By J. E. GraY. $8^{\circ}$. 1870 .

Lists of British Animals.

List of British Birds. By G. R. Gray. $12^{\circ}$. 1850. " Eggs of British Birds. By G. R. Gray.

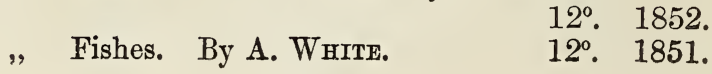


MUSEUM (BRITISH), continued.

List of Mollusca. By J. E. Gray.

$12^{\circ} .1851$.

" Crustacea. By A. White.

$8^{\circ} .1850$.

, Lepidoptera. (Part V. of the series.) By J.F. STEPHENs. $12^{\circ}$. 1850. 2nd edition (by H. T. Statnton \& E. Shepherd). 1856. $" \quad, \quad$ (continued.) - (Part X.) 1852. $" \quad, \quad$ (continued.) (Part XII.) 1852. H. T. Stainton. (completed.) (Part XVI.) By

" Hymenoptera. By F. Suiтr. $\quad 8^{\circ} .1851$.

Catalogue of British Hymenopterc. Part I. Apida. By F. Simth.

$12^{\circ} .1855$.

2nd edition. $8^{\circ} .1876$.

Catalogue of British Fossorial Hymenoptera (Formicidce and Vespidce). By F. Suiтh. $\quad 12^{\circ}$. 1858. List of Anoplura. By H. Denny. $12^{\circ}$. 1852.

" Curculionidæ. By J. Walton. 12 1856.

" Ichneumonidæ. By T. Desvignes. $12^{\circ} .1856$.

Nomenclature of Hymenoptera. By F. Sмгтн. 1853.

" Neuroptera. By A. White. 1853.

„ Diptera. By A. White. 1853.

, Anoplura. By A. Whте. 1855.

List of Centronia, or Radiated Animals. By J. E. Gray. $8^{\circ} .1848$. " Sponges. By J. E. Grar. $12^{\circ}$. 1848.

Synopses AND GUIDES.

Synopsis of the British Museum. 9th ed. $8^{\circ}$. 1815.

$\begin{array}{lllll}" & " & \text { 26th ed. } 8^{\circ} . & 1832 . \\ " & " & \text { 28th ed. } & 8^{\circ} . & 1834 . \\ " & " & \text { 29th ed. } & 8^{\circ} . & 1835 . \\ " & " & \text { 42nd ed. } & 8^{\circ} . & 1840 . \\ \prime & & \text { 43rd ed. } & 8^{\circ} . & 1841 .\end{array}$

Synopsis, or Guide-Book, to the British Museum. Part I. Zoology. $\quad 12^{\circ} .1843$. Another edition. $12^{\circ} .1848$.

Guide to the Exhibition Galleries of the British Museum, Bloomsbury.

$8^{\circ}$. 1882 .

Guide to the Collection of Meteorites in the Department of Mineralogy.

$8^{\circ} .1882$. 
MUSEUM (BRITISH), continued.

Index to the Collection of Minerals.

$8^{\circ} .1882$.

Guide to the Gould Collection of Humming-Birds.

$8^{\circ} .1883$.

2nd edition. $\dot{8}^{\circ} .1883$.

3rd edition. $8^{\circ} .1884$.

Guide to the Exhibition Galleries of the Department of Geology and Palæontology. British Museum (N.H.).

$8^{\circ}$. 1882.

A Guide to the Exhibition Galleries in the British Museum (N. H.). Departments of Geology and Palæontology, Mineralogy, and Botiny. $8^{\circ}$. 1883.

MUSEUM (CALCUTTA). Catalogue of the Mammalia in the Museum of the Asiatic Society, Calcutta. By E. BLYTH.

Catalogue of the Birds. By E. Buytr.

8. Calcutta, 1863.

$8^{\circ}$. Calcutta, 1849.

Catalogue of the Mollusca.

By G. Neville.

$8^{\circ}$. Calcutta, 1877.

Reports of the Curator. $\quad 8^{\circ}$. Calcutta, 1847-59.

MUSEUal CaLonniandM. See Calonne (De).

MUSEUM (CAMBRIDGE). Catalogue of the Osteological Specimens in the University of Cambridge.

8. Cambridge, 1862.

Catalogue of the Strickland Collection of Birds in the University of Cambridge. By O. SALviN.

$8^{\circ}$. Cambridge, 1882.

MUSEUM (CHATHAM). Catalogue of the Mammals and Birds in the Museum, Fort Pitt, Chatham.

Catalogue of the Reptiles.

8. Chatham, 1838.

8. Chatham, 1843.

MUSEUM (COLLEGE OF SURGEONS, LONDON).

Сов воLd (T.S.). Catalogue o: the|Specimens of Entozoa. 8. London, 1866.

Flower (W. H.). Catalogue of Specimens illustrating the Osteology and Dentition of Vertebrated Animals.

Part I. $8^{\circ}$. London, 1879.

Part II. $8^{\circ}$. London, 1884.

Honter (J.). Observations and Reflections on Geo$\log y$. $4^{\circ}$. London, 1859. 
MUSEUM (COLL. OF SURGEONS, LOND.), continued. Hunter (J.). Memoranda on Vegetation.

$4^{\circ}$. London, 1860.

Lowne (B. T.). Descriptive Catalogue of the Teratological Series. $\quad 8^{\circ}$. London, 1872.

Morris (J.) \& OWen (R.). Descriptive Catalogue of the Fossil Organic Remains of Invertebrata.

$4^{\circ}$. London, 1856.

Owen (R.). Description of a Skeleton of an Extinct Gigantic Sloth.

$4^{\circ}$. London, 1842.

Owex (R.). Descriptive Catalogue of the Physiological Series of Comparative Anatomy.

2nd edition. $8^{\circ}$. London, 1852.

Owen (R.). Descriptive Catalogue of the Osteological Series. 2 vols. $4^{\circ}$. London, 1853.

Owen (R.). Descriptive Catalogue of the Fossil Organic Remains of Reptilia and Pisces.

8. London, 1854.

Paget (J.), Goodhart (J. F.), \& Doran (J.). Descriptive Catalogue of the Pathological Specimens.

2nd edition. $8^{\circ}$. London, 1882.

Queketr (J.). Descriptive and Illustrative Catalogue of Elementary Tissues of Vegetables and Animals.

$4^{\circ}$. London, 1850.

QUeкetT (J.). Catalogue of Plants and Invertebrate Animals in a Dried State. $\quad 4^{\circ}$. London, 1860.

Taylor (T.). Descriptive and Illustrated Catalogue of the Calculi and other Animal Concretions.

$4^{\circ}$. London, 1842--45.

Supplement. $4^{\circ}$. London, 1871.

MUSEUMI (COPENHAGEN). See JACobæUs (0.).

MUSEUM GODEFFROY, Journal des. Geographische, Ethnographische, und Naturwissenschaftliche Mittheilungen. Hefte I. $-\mathrm{XV}$.

$4^{\circ}$. Hamburg, 1873-81.

Catalog III.-VII.

$8^{\circ}$. Hamburg, 1866-79.

MUSEUM GOTTWALDIANUM. See Schröter (J. S.).

MUSEUM (HAJBURG). See MöвIUs (K.). 
MUSEUM (HARVARD COLLEGE, CAMBRIDGE, U.S.).

Bulletin of the Museum of Comparative Zoology. Vols. I.-XI. $8^{\circ}$. Cambridge, U.S., 1863-83.

Memoirs of the Museum of Comparative Zoology.

Vols. I.-IX. $\quad 4^{\circ}$. Cambridge, U.S., 1865-83.

MUSEUAI HEINEANUM. See CABANIS (J.).

MUSEUM (INDIA). Catalogue of the Mammalia in the Museum of the Hon. East-India Company. By T. HoRSFIELD.

2 vols. $8^{\circ}$. London, 1851.

Catalogue of the Birds. By T. Horsfield \& F. Moore.

2 vols. $8^{\circ}$. London, 1854.

Catalogue of the Lepidopterous Insects. By T. HorsFIELd \& F. MLoore. 2 vols. $8^{\circ}$. London, 1857-59.

Catalogue of the Mollusca. By G. Nevilu.

8. Calcutta, 1877.

MUSEUM KIRChERIANUM. See Bonnani (P. P.).

MUSEUM LESKEANUM. See Karsten (L. G.).

MUSEUMI LEVERIANUM. See Shaw (G.).

MUSEUM (LEYDEN). MIuséum d'Histoire naturelle des Pays-Bas. Par H. Schieg fer. 3 vols. $8^{\circ}$. Leyde, 1862.

Table Analytique. Par F. A. Jentinrs.

8. Leyde, 1881.

Notes from the Royal Zoological Museum of the Netherlands at Leiden. Edited by H. SchlegeL.

5 vols. $8^{\circ}$. Leiden, 1879-83. [In progress.]

MUSEUM (MILAN). See JAN ( $\left(_{*}\right)$.

MUSEUM (NAPLES). See Costa (A.) \& Panceri (P.).

MUSEUM (PARIS). Muséum d'Histoire Naturelle de Paris. Catalogue des Mammifères. Par G. Str. Hilarre, Prérost, et Puchéran. $\quad 8^{\circ}$. Paris, 1851.

Catalogue des Coléoptères. Par A. Milnek-Edwards, E. Bianchard, et H. Lucas. $\quad 8^{\circ}$. Paris, 1850.

Rérision de la collection de Stellérides par J. O. E. PERRIER.

8. Paris, 1875.

See Deleuze (M.). 
MUSEUM SENCKENBERGIANUM. Abhandlungen aus dem Gebiete der beschreibenden Naturgeschichte.

$4^{\circ}$. Franlfurt am $M$.

Verzeichniss der in dem Museum der Senckenbergischen naturforschenden Gesellschaft aufgestellten Sammlungen. Von E. RÜPPELL.

$4^{\circ}$. Frankfurt, 1842.

Abth. I. Säugethiere und deren Skelette. 1842.

"II. , , ,

"III. Amphibien, 1845.

"IV. Fische und deren Skelette.

1852.

Öffentliche Rede, nebst einer Beschreibung und Abbildung mehrerer in dem Gesellschafts-Museum aufgestellten interessanten fossilen Reptilien. Von E. RüPPELL. $4^{\circ}$. Fronkfurt, 1845.

MUSEUM OF SMITHSONIAN INSTITUTION. Seo Smithsonian Institution.

MUSEUM (STOCKHOLM). See LinNæUs (C.).

MUSEUM (VERONA). Cataiogus systematicus rerum Naturalium in Museo extantium. Sectio 1. Reptilia Europæ. E. DE BetTa.

$8^{\circ}$. Veronce, 1853.

MUSEUM (WARSAW). See Gravenhorst (J. L. C.).

MUSEUM (-WELLINGTON, N.Z.). Catalogue of the Colonial Museum, Wellington, New Zealand.

$12^{\circ}$. Wellington, 1870.

Catalogue of the New Zealand Diptera, Orthoptera, and Hymenoptera. By F. W. Hutron.

$8^{\circ}$. Wellington, 1881.

Reports of Geological Explorations during 1881. By J. HeCTOR.

$8^{\circ}$. Wellington, 1882 .

Seventeenth Annual Report on the Colonial Museum and Laboratory. By J. Hector.

$8^{\circ}$. Wellington, 1882.

MUSEUM (ZOOLOGICAL SOCIETY). Catalogue of the Mammalia contained in the Museum of the Zoological Society of London.

So. London, 1828. 
MUSEUM (ZOOLOGICAL SOCIETY), continued.

Catalogue of the Animals preserved in the Museum of the Zoological Society of London.

8. London, 1829.

List of the Animals in the Gardens of the Zoological Society of London. $\quad 8^{\circ}$. London, 1830.

Supplement to the Catalogue of the Mammalia preserved in the Museum of the Zoological Society of London.

8. London, 1839.

Revised list of the Vertebrated Animals now or lately living in the Gardens of the Zoological Society of London.

8. London, 1872.

The same. 7 th edition. $8^{\circ} . \quad$ London, 1879.

The same. 8th edition. $8^{\circ}$. London, 1883.

MUSGRAVE (T.). Cast away on the Auckland Isles. post $8^{\circ}$. London, 1866.

N.

NARDO (J. D.). Prodromus observationum et disquisitionum Adriaticæ Ichthyologiæ. $\quad 4^{\circ}$. Ticini Regii, 1827.

NARDO (G. D.). Considerazioni sulla famiglia dei pesci Mola, e sui caratteri che li distinguono.

$4^{\circ} . \quad$ Padova, 1840.

NARDO (G. D.). Osservazioni anatomiche comparative sull' intima struttura delle cartilagini dei Chondrotterigi.

$4^{\circ}$. Venezia, 1843.

NARDO (G. D.). Prospetto della Fauna Marina volgare del Veneto Estuario.

8․ Venezia, 1847.

NARDO (G. D.). Sopra due Specie dei Pesci pubblicate come nuove dal Prof. R. Molin. $\quad 8^{\circ}$. Venezia, 1853.

NARDO (G. D.). Prospetti sistematici degli Animali delle Provincie Venete.

8․ Venezia, 1860.

NARDO (G. D.). Cenni Storico-Critici.

$8^{\circ}$. Tenezia, 1875. 
NASSE (0.). See Hermand (L.). Handbuch der Physiologie.

NATHUSIUS (H. von). Vorstudien für Geschichte und Zucht der Hausthiere, zunaechst am Schweineschaedel. Text, $8^{\circ}$. Atlas, obl. $4^{\circ}$. Berlin, 1864.

NATHUSIUS (H. von). Öber die sogenannten Leporiden. 8. Berlin, 1876.

NATHUSIUS-KOENIGBORN (W. von). Untersuchungen über nicht Celluläre Organismen, namentlich CrustaceenPanzer, Mollusken-Schalen, und Eihüllen.

$4^{\circ}$. Berlin, 1877.

NAUMANN (J. A.). Naturgeschichto der Vögel Deutschlands. 13 vols. $8^{\circ}$. Leipzig, 180-60.

NAUMANN (J. F.) \& BUHLE (C. A.). Die Eier der Vögel Deutschlands. $4^{\circ}$. Halle, 1818-28.

NAUMANN (J. F.). Ueber den Haushalt der Nordischen Seevögel Europa's. obl. fol. Leipzig, 1824.

NECKER (L. A.). Mémoire sur les Oiseaux des environs de Genève.

$4^{\circ}$. Genève et Paris, 1823.

NEERGAARD (J. W.). Vergleichende Anatomie und Physiologie der Verdauungswerkzeuge der Säugethiere und Vögel. Vorrede von J. F. Blumenbach. 80. Berlin, 1806.

NEES VON ESENBECK (C. G.). Hymenopterorum Ichneumonibus affinium Monographiæ.

2 vols. $8^{\circ}$. Stuttgart, 1834.

NEGRO. Dissertation physique à l'occasion du Nègre Blanc. [Anon.] 12 . Leyde, 1744.

NEICKELIO (C. F.). Museographia.

$4^{\circ}$. Leipzig \& Breslau, 1727.

NEITZSCHÜTZ (M. จ.). Studien zur Entwickelungsgeschichte des Schafes.

8. Danzig, 1879.

NELSON (E. W.). See 'CoRwin.' 
NELSON (W.). The Laws concerning Game.

8. London, 1751.

NENNING (S.). Die Fische des Bodensees, nach ihrer äussern Erscheinung.

8. Konstanz, 1834.

NEUMAN (C. J.). Om Sreriges Hydrachnider.

$4^{\circ}$. Stockholm, 1880.

NEUMAN \& BARETTI. See Dictionaries.

NEUMEYER (G.). Anleitung zu Wissenschaftlichen Beobachtungen auf Reisen.

$8^{\circ}$. Berlin, 1875.

NECSTAEDT (A.) \& KORNATZKI (C.). Abbildung und Beschreibung der Schmetterlinge Schlesiens in systematischer Ordnung. $\quad 2$ rols. $4^{\circ}$. Breslau, 18+2-45.

NEVILL (G.). Catalogue of Mollusca in the Indian Museum, Calcutta.

8. Calcutta, 1877.

See Museum (Calcutta).

NEVILL (G.). See Strveys \& Explorations: Yarkand.

NEWALL (J. T.). The Eastern Hunters.

8. London, 1866.

NEWMAN (E.). Sphinx Vespiformis.

8. London, 1832.

NEWMAN (E.). The System of Nature.

2 parts. $8^{\circ}$. London, 1842-43.

NEWMAN (E.). British Moths. 80. London, 1869-74.

NEWMAN (E.). British Butterflies. $8^{\circ}$. London, n. d.

NEWMAT (E.). See Montagu (G.).

NEWMAN (E.). See Periodicals : London.

NEWPORT (G.). See MuseuM (British). 
NEWTON (A.). A List of the Birds of Europe. By Prof. J. H. BLasidus. 8. Norwich, 1862.

NEWTON (A.). See Periodicals: London. 'The Ibis' and 'Zoological Record.'

NEWTON (A.). See Wolley (J.).

NEWTON (A.). See YARRELL (W.).

NICHOLSON (E.). Indian Snakes. 2nd edition. 8o. Madras, 1874.

NICHOLSON (H. A.). Manual of Zoology. 5th edition. $8^{n}$. London, 1878.

NICHOLSON (H. A.). An Introductory Text-Book of Zoology for the Use of Junior Classes.

5th edition. $8^{\circ}$. Edinb. \& Lond., 1881.

NICHOLSON (H. A.). Synopsis of the Classification of the Animal Kingdom. $\quad 8^{\circ}$. Edinb: \& Lond., 1882.

NICKERL (F. A.). Böhmens Tagfalter.

8. Prag, 1837.

NICOLAI (E. A.). Coleopterorum species Agri Halensis. $8^{\circ}$. Halce, 1822.

NIEBUHR (C.). See ForsKåc (P.).

NILSSON (L. G.), WIDMARK (P. F.), \& COLLIN (A. Z.). See Dictionartes: Engelsk-SvensK.

NLLSSON (S.). Ornithologia Svecica.

2 vols. $8^{\circ}$. Havnice, 1817.

NILSSON (S.). Historia Molluscorum Sreciæ.

8.. Lund, 1822.

NILSSON (S.). Illuminerade Figurer till Skandinaviens Fauna.

2 vols. $4^{\circ}$. Lund, 1829-40. 
NILSSON (S.). Prodromus Ichthyologiæ Scandinavicæ.

(Interleaved with MS. notes, $4^{\circ}$.)

8. . Lundae, 1832.

NILSSON (S.). Scandinavisk Fauna.

Däggdjuren. $\quad 1$ vol. $8^{\circ}$. Lund, 1847.

Foglarna. 2 vols. 2nd edition. $8^{\circ}$. Lund, 1835.

Amfibierna.

2 vols. 3rd edition. $8^{\circ}$. Lund, 1858.

1 vol. $8^{\circ}$. Lund, 1842.

Fiskarna.

1 vol. 2nd edition. $8^{\circ}$. Lund, 1860.

1 vol. $8^{\circ}$. Lund, 1855.

NITSCHE (H.). See Fisheries : Beruin. Internationale Fischerei-Ausstellung, 1880.

NITSCHE (H.). See Ledckart (R.) \& Nitsche (H.).

NITZSCH (C. L.). Osteografische Beiträge zur Naturgeschichte der Vögel.

8. Leipzig, 1811.

NITZSCH (C. L.). Beobachtungen über Thierwürmer.

Vols. II., III. $\quad 4^{\circ}$. 1814-15.

NITZSCH (C. L.). Beitrag zur Infusorienkunde.

8. Halle, 1817.

NITZSCH (C. L.). Darstellung der Familien und Gattungen der Thierinsekten (Insecta Epizoica).

12․ Halle, 1818.

NITZSCH (C. L.). Spiropteræ strumosæ descriptio.

$4^{\circ}$. Halce, 1829.

NITZSCH (C. L.). Pterylographia Avium. Pars I.

See RAy Societr.

$4^{\circ}$. Halce, 1833.

NITZSCH (C. L.). System der Pterylographie. Verfasst von H. BURMEIsTER.

$4^{\circ}$. Halle, 1840.

NODDER (F. P.). See Shaw (G.) \& Nodder (F. P.).

NOEHRING (J.) \& LENZ (H.). Die anthropomorphen Affen des Lübecker Museums.

$4^{\circ}$. Lübeck, 1876. 
NOEL (A.). Collection Entomologique ou Histoire Naturelle des Insectes peints par A. Nö̈L et gravés sous la direction de M. Pauquez. 8. . Paris, 1830.

NOIRMONT (Baron Dunoyer de). Histoire de la Chasse en France. 3 vols. $8^{\circ}$. Paris, 1867-68.

NOLL (F. C.). See Periodicals: Frankfurt a. M.

NÖRDLINGER (H.). Nachträge zu Ratzenburg's Forstinsekten. 8. Stuttgart, 1856.

NÖRDLINGER(H.). Die kleinen Feinde der Landwirthschaft.

$8^{\circ}$. Stuttgart, 1855. 2nd edition. $8^{\circ}$. Stuttgart, 1869.

NORDMANN (A. von). Mikrographische Beiträge zur Naturgeschichte der wirbellosen Thiere.

$4^{\circ}$. Berlin, 1832.

NORDMANN (A. von). Symbolæ ad Monographiam Staphylinorum. $4^{\circ}$. Petropoli, 1837.

TORDMANN (A. von). Versuch einer Natur- und Entwickelungsgeschichte des Tergipes Edwardsii.

$4^{\circ}$. St. Petersburg, 1845.

NORDSTRÖM (C.). Osteologia Hyænæ. [In Russian]. gr. $4^{\circ}$. St. Petersburg, 1859.

NORGUET (A. de). Catalogue des Coléoptères du Département du Nord. $\quad 8^{\circ}$. Lille, 1863.

NORMAN (A. M.). Monograph of the British Spongiida. See Ray Society.

NORRIS (E.). See Prichard (J. C.).

NORTON (E.). See Surteys \& Explorations: U.S., WASHINGTON.

NORWEGLAN. North Atlantic Expedition. See Survers \& Explorations: Norwar. 
NOTT (J. C.) \& GLIDDON (G. R.). Types of Mankind. 8th edition. 8. Philadelphia, 1857. 10th edition. $8^{\circ}$. Philadelphia, 1871.

NOTT (J. C.) \& GLIDDON (G. R.). Indigenous Races of the Earth.

8. Philadelphia, 1868.

NOULET (J. B.). Précis analytique de l'Histoire Naturelle des Mollusques terrestres et fluviatiles qui vivent dans le Bassin Sous-Pyrénéen. $\quad 8^{\circ}$. Toulouse, 1834.

' NOVARA.' Reise der österreichischen Fregatte ' Novara' um die Erde in den Jahren 1857, 1858, 1859.

Säugethiere. Johaxy Zelebor.

4. TVien, 186t-75.

Vögel. A. vor Pelzelis. 1868 .

Reptilien. F. Stemndachner.

1865.

Amphibien. F. Steindachier.

Fische. R. Kren.

1867.

Mollusken. G. Pitter v. Fradenfeld.

1867.

Crustaceen. C. Heller.

$1865-67$.

Coleopteren. L. Redtenbicher.

Hymenopteren. H. JE SAUssure.

Lepidopteren. C. u. R. FELDER.

Hemipteren. G. L. MAYR.

Neuropteren. F. Brader.

Formiciden. G. L. MaYR.

1867.

1865 .

1867.

1867.

Dipteren. J. R. Schlier.

Anneliden. E. Grube.

$1874-75$.

1866.

1866.

1865.

1868.

1867.

NOVITATES CONCHOLOGIC $\mathrm{E}$. Abbildung und Beschreibung neuer Conchylien.

Tols. 1-5. By Dr. Louis Pfeiffer.

Supplements :

$4^{\circ}$. Cassel, 1854-79.

I. Monographie der Mollusken Gattung Dosinia. Von E. Röyer. $4^{\circ}$. Cassel, 1862.

II. a. Index Molluscorum quæ in itinere ad Guineam inferiorem collegit G. Tams. Auctore G. Duxker. $\quad 4^{\circ}$. Cassel, 1853.

II. $b$. Abbildung und Beschreibung neuer Conchylien. II. Abtheilung. Meeres Conchylien. Von W. DUNKer. $\quad 4^{\circ}$. Cassel, 1858-70.

III. Monographie der Mollusken-Gattung Venus. Vor E. Rösrer. $\quad 4^{\circ}$. Cassel, 1869.

IV. Japanische Meeres-Conchylien. Von C. E. Lischke. $\quad 4^{\circ}$. Cassel, 1869-74. 
NOVITATES CONCHOLOGIC.E, continued.

V. Ueber Vorderasiatische Conchylien. Von E. von Martens. $4^{\circ}$. Cassel, n. d.

VI. Clausilien-Studien. Von O. Bofttakr.

$4^{\circ}$. Cassel, 1877.

VII. Index Molluscorum Maris Japonici. Auct. G. DUNKer. $\quad 4^{\circ}$. Cassel, 1882.

NOWICKI (M.). Enumeratio Lepidopterorum Haliciæ Orientalis.

8. Leopoli, 1860.

NOWICKI (M.). Microlepidopterorum Species Novæ.

See Nowickiego (М.).

8. Cracovice, 1864.

NOWICKI (MI.). Beiträge zur Lepidopterenfauna Galiziens. 8. Krakau, 1865.

NOWICKI (M.). Motyle Galicyi (Rhopalocera).

8. We Lwowie, 1865.

NOWICKI (M.). Wykaz motylów tatrzanskich wedlug pionowego rozsiedlenia. [Lepidoptera.]

8. Krakow, 1868.

NOZEMAN (C.). Nederlandsche Vogelen.

5 vols. fol. Amsterdam, 1797.

NUÑEZ (J.). Etude médicale sur le Venin de la Tarentule.

8. Paris, 1866.

NUNN (J.). Narrative of the Wreck of the 'Favorite' on the Island of Desolation. Edited by W. B. Clarke.

8. London, 1850.

NÜSSLIN (O.). Zur Kritik des Amphioxus Auges.

8. Tübingen, 1877.

NUTTALL (T.). A Manual of the Ornithology of the United States and of Canada.

2 vols. $\quad 8^{\circ}$. Cambridge, Mass., 1832-34.

NYLANDER (A. E.). Finlands Mollusker.

$8^{\circ}$. Helsingfors, 1856.

ZOOL. CAT. 
0 .

OATES (E. W.). A Handbook to the Birds of British Burmah.

2 vols. roy. $8^{\circ}$. London, 1883.

OBERT (J.). Catalogus Coleopterorum Agri Petropolitani.

8. Petropoli, 1875.

OBERTHÜR (C.). Etudes d'Entomologie. Faunes Entomologiques: descriptions d'Insectes nouveaux ou peu connus.

Parts 1-7. 80. Rennes, 1876-83.

OBERTHÜR (C.). Diagnoses d'espèces nouvelles de Lépidoptères d'île Askold.

8. Rennes, 1879.

OBERTHÜR (R.). Coleopterorum Novitates.

Vol. i. pt. 1. roy. $8^{\circ}$. Paris, 1883.

[In progress.]

OBSONVILLE (F. L. d'). Essais philosophiques sur les mœurs de divers animaux étrangers, .... ou Extraits des voyages de $\mathrm{M} * * *$ en Asie. $\quad 8^{\circ}$. Paris, 1783.

OCHSENHEIMER (F.). Die Schmetterlinge Sachsens. 8. Dresden \& Leipzig, 1805.

OCHSENHEIMER (F.) \& FREITSCHKE (F。). Diө Schmetterlinge von Europa.

14 vols. 8. Leipzig, 1807-35.

OERSTED (A. S.). Entwurf einer systematischen Eintheilung und speciellen Beschreibung der Plattwürmer.

8. Copentagen, 1844.

OERSTED (A. S.). Annulatorum Danicorum Conspectus. 8. Hafnice, 1843.

OFSIANNIKOF. See Owsjannmow。

OHLERT (E.). Die Araneiden der Provinz Preussen.

8. Leipzig, 1867. 
OKEN (L.). Lehrbuch der Naturphilosophie.

3 vols. in 2. $8^{\circ}$. Jena, 1809-11.

OKEN (L. v.). Allgemeine Naturgeschichte für alle Stände. 7 vols. $8^{\circ}$. Atlas, $4^{\circ}$. Stuttgart, 1839-43.

OKOSHI (Narinori). See Fisheries : London. International Fisheries Exhibition, 1883.

OLAUS MAGNUS. Gentium Septentrionalium Historiæ Breviarium. 12. Lugduni Batavorum, 1652.

OLEARIUS (A.). Gottorfische Kunst Kammer.

$4^{\circ}$. Schleswig, 1674.

OLFERS (J. F. M. von). Ueber eine neue Art Seeblase (Physalia producta).

$4^{\circ}$. Berlin, 1820-21.

OLFERS (J. F. M. von). Ueber die grosse Seeblase (Physalia arethusa).

$4^{\circ}$. Berlin, 1832

OLINA (G. P.). Uccelliera.

$4^{\circ} . \quad R o m a, 1684$.

OLIVI (G.). Zoologica Adriatica. $\quad 4^{\circ} . \quad$ Bassano, 1792.

OLIVIER (E.). La Chrysomèle des pommes de terre. 2nd edition. $12^{\circ}$. Besançon, 1878.

OLIVIER (G. A.). Entomologie.

8 vols. $4^{\text {n. }} \quad$ Paris, 1789.

OLSEN (J.). Icththyographia Islandica.

MS. $4^{\circ}$. Kjöbenhavn, 1737.

OLSEN (O. T.). The Fisherman's Seamanship.

8. Lonclon, 1882.

OLSEN (O. T.). The Piscatorial Atlas of the North Sea, English and St. George's Channels.

fol. Grimsby \& London, 1883. 
OLTMANS (A.). Catalogue des Coquilles de la Famille des Conides, qui se trouvent au Musée de la Société Zoologique d'Amsterdam.

$4^{\circ}$. 1869 .

See Musedm (Amsterdair).

ÖMAN (V. E.). See Dictionaries : Svensk-Engelsk.

OPPEL (M.). Die Ordnungen, Familien, und Gattungen der Reptilien. $4^{\circ}$. München, 1811.

ORBIGNY (A. d'). Mollusques aux Iles Canaries.

$4^{\circ}$. Paris, 1842 .

ORBIGNY (A. d'). Prodrome de la Paléontologie des Animaux Mollusques et Rayonnés.

2 vols: $8^{\circ}$. Puris, 1849-52.

ORBIGNY (A. d'). Cours élémentaire de Paléontologie et de Géologie stratigraphiques.

2 vols. $8^{\circ}$. Paris, 1849-52.

ORBIGNY (A. d'). Mollusques Tivants et Fossiles.

8. Paris, 1855.

ORBIGNY (A. d'). Shells of the Canaries.

See Museum (Brttish).

ORBIGNY (A. d'). Shells of Cuba.

See Musedx (British).

ORBIGNY (A. d'). Shells of South America.

See Museum (British).

O'REILLY (B.). Greenland, the adjacent Seas, and the North-west Passage to the Pacific. $\quad 4^{\circ}$. London, 1818.

ORIENTREISE (Eine). [By the Archduke RudoLPH, Crown Prince of Austria.] $\quad 8^{\circ}$. Wien, 1881.

ORMEROD (E. A.). A Manual of Injurious Insects.

$8^{\circ}$. Loncton, 1881.

ORMEROD (E. L.). British Social Wasps.

8. London, 1868. 
ORZA (P. de L'). Les Lépidoptìres Japonais à la grande Exposition Internationale de $1867 . \quad 88^{\circ}$. Paris, 1869.

OSCULATI (G.). Note d' un Viaggio nella Persia e nelle Indie Orientali, 1841-42.

8. Monza, 1844.

OSTEN-SACKEN (C. R.). Catalogue of the described Dipterc of North America. $\quad 8^{\circ}$. Washington, 1858.

OSTEN-SACKEN (C. R.). Monographs of the Diptera of North America.

Parts I. \& II. 8º. Washington, 1862-64. See Smithsonian Miscellaneods Collections.

OSTEN-SACKEN (C. R.). Report upon the Collection of Diptera. $4^{\circ}$. Washington, 1873.

OSTEN-SACKEN (C. R.). Western Diptera.

8. Washington, 1877.

OSTEN-SACKEN (C. R.). See Surveys \& Explorations: U.S., WASHINGTON.

OTTO(A.G.). Animalium Maritimorum nondum editorum Genera Duo. I. Sternaspis thalassemoides. II. Siphostoma diplochaitus.

4.. Bonnce, 1821.

OTTO (A. G.). De rarioribus quibusdam sceleti humani cum animalium sceleto Analogiis. $4^{\circ}$. Vratislavice, 1839.

OTTO (B. C.). Conspectus Animalium quorundam maritimorum.

4․ Vratislavice, 1821.

OUSTALET (E.). Monographie des Oiseaux de la Famille des Megapodiides. 8. Paris, 1881.

OWEN (C.). An Essay towards a Natural History of Serpents. $4^{\circ}$. London, 1742.

OWEN (R.). Memoir on the Pearly Nautilus.

$4^{\circ}$. London, 1832.

OWEN (R.). Odontography.

2 rols. $8^{\circ}$. London, 1840-45. 
OWEN (R.). Lectures on the Comparative Anatomy of the Invertebrate Animals. $\quad 8^{\circ}$. London, 1843. 2nd edition. 8\%. London, 1855.

OWEN.(R.). Lectures on the Comparative Anatomy of the Vertebrate Animals. Part I. Fishes.

8. . London, 1846.

OWEN (R.). On the Nature of Limbs.

8. London, 1849.

OWEN (R.). Monograph on the Fossil Reptilia of the London Clay.

$4^{\circ}$. London, 1850.

OWEN (R.). Mollusca. From the Encyclopædia Britannica, 8th edition.

$4^{\circ}$. Edinburgh, 1858.

OWEN (R.). On the Classification and Geographical Distribution of the Mammalia. $\quad 8^{\circ}$. London, 1859.

OWEN (R.). Anatomy of the Vertebrates.

3 vols. $8^{\circ}$. London, 1866-68.

OWEN (R.). Descriptive and Illustrated Catalogue of the Fossil Reptilia of South Africa in the British Museum. $4^{\circ}$. London, 1876.

OWEN (R.). See 'Beagle.'

OWEN (R.). See 'Blossoy.'

OWEN (R.). See Hunter (J.).

OWEN (R.). See Museum: College of Surgfors.

OWSJANNIKOW (P.). Ueber das Gehörorgan von Petromyzon fluviatitis. 4\%. St. Petersburg, 1861.

OWSJANNIKOW (P.). Ueber die feinere Structur der Kopfganglions bei den Krebsen, besonders beim Palinurus locusta. $4^{\circ}$. St. Petersburg, 1863. 
OWSJANNIKOW (P.). Ein Beitrag zur Kenntniss der Leuchtorgane von Lampyris noctiluca.

$4^{\circ}$. St. Petersburg, 1868.

OWSJANNIKOW (P.). Ueber die Rinde des Grosshirns beim Delphin und einigen anderen Wirbelthieren.

$4^{\circ}$. St. Petersburg, 1879.

OWSJANNIKOW (P.) \& KOWALEWSKY (A.). Ueber das Centralnervensystem und das Gehörorgan der Cephalopoden.

$4^{\circ}$. St. Petersburg, 1867.

P.

PACKARD (A. S.). Record of American Entomology for the year 1868 .

$8^{\circ}$. Salem, 1869.

PACKARD (A. S.). Monograph of the Geometrid Moths of the United States. $\quad 4^{\circ}$. Washington, 1876.

See Survers \& Explorations: U.S., Washington.

PACKARD (A. S.). Guide to the Study of Insects. 8. New York, 1880.

PACKARD (A. S.). Zoology for High Schools and Colleges. 3rd edition. $8^{\circ}$. New York, 1881.

PACKaRd (A. S.). See Periodicals: Washington.

PACKARD (A. S., Jun.). On the Rocky Mountain Locusts. $8^{\circ}$. Washington, 1878.

See Survers \& Explorations: U.S., Washington.

PAETEL (F.). Catalog der Conchylien.

$8^{\circ}$. Berlin, 1873 .

PAETEL (F.). Die Familien- und Gattungsnamen der Mollusken.

$8^{\circ}$. Berlin, 1875.

PAETEL (F.). Catalog der Conchylien Sammlung. ro5. $8^{\circ}$. Berlin, 1823. 
PAGENSTECHER (H. A.). Beiträge zur Anatomie der Milben. fol. Leipzig, 1860-61.

PAGENSTECHER (H. A.). Die Trichinen. 2nd edition. 8०. Leipzig, 1866.

PAGENSTECHER (H. A.). Allgemeine Zoologie.

8. Berlin, 1875-81.

PAGENSTECheR (H. A.). See Kossurand (R.).

Paget (Sir J.). See Musedx: College of Surgeons.

PAHLEN (A. von der). Monographie der baltisch-silurischen Arten der Brachiopoden-Gattung Orthisina.

$4^{\circ}$. St. Petersburg, 1877.

PAIVA (C. de). Insectos Coleopteros de Camboja.

8․ Lisboa, 1860.

PALISOT DE BEAUVOIS (A. M. F. J.). Insectes recueillis en Afrique et en Amérique. fol. Paris, 1805.

PALLAS (P. S.). Miscellanea Zoologica.

4․ Hagce, 1766.

PALLAS (P. S.). Elenchus Zoophytorum.

8. Hagoe, 1766.

PALLAS (P. S.). Spicilegia Zoologica.

$4^{\circ}$. Berolini, 1767-80.

PALLAS (P. S.). Naturgeschichte merkwürdiger Thiere.

2 vols. $4^{\circ}$. Berlin, 1769-79.

PALLAS (P. S.). Icones Insectorum.

$4^{\circ}$. Erlangce, 1781.

PALLAS (P. S.). Charakteristik der Thierpflanzen. 2 vols. $4^{\circ}$. Niirnberg, 1787.

PALLAS (P. S.). Neue Nordische Beyträge.

7 vols. $8^{\circ}$. St. Petersburg, 1781-96. 
PALLAS (P. S.). Novæ species Quadrupedum. 4\%. Erlangae, 1798.

PALLAS (P. S.). Zoographia Rosso-Asiatica. 3 vols. $4^{\text {o. }}$ Petropoli, 1811-31.

PALLIARDI (A.). Beschreibung zweyer Decaden neuer und wenig bekannter Carabicinen. $\quad 8^{\circ}$. Wien, 1825.

PALMÉN (J. A.). Ueber die Zugstrassen der Vögel.

8. Leipzig, 1876.

PALMEN (J. A.). Antwort an Herrn E. F. von Homeyer bezüglich der "Zugstrassen der Vögel."

8․ Helsingfors \& Leipzig, 1882.

PALMIERI (A.). Dizionario Portatile a fugare i principali Insetti.

16. Foligno, 1835.

PALMSTRUCH (J. W.). See Svensk Zoologi.

Palmstruch (J. W.). See Quensel (C.), Palmstruch (J. W.), \& SWARTZ (O.).

PANCERI (P.). Catalogo sistematico del Gabinetto nella Regia Università degli Studi di Napoli.

$8^{\circ}$ (with Supplement). Napoli, 1868.

PANCERI (P.) \& SANCTIS (L. de). Sopra alcuni organi della Cephaloptera giorna.

4\%. Napoli, 1869.

PANDER (C. H.) \& ALTON (E. d'). Vergleichende Osteologie. obl. fol. Bonn, 1821-38.

PANICELLI (C.). Trattato degl' effetti maravigliosi delle Carni di Vipere.

$8^{\circ}$. Fiorenza, 1630.

PANZER (G. W. F.). Faunæ Insectorum Americes Borealis Prodromus. $\quad 4^{\circ}$. Norimberga, 1794.

PANZER (G. W. F.). Deutschlands Insectenfaune, oder entomologisches Taschenbuch für das Jahr 1795.

$12^{\circ}$. Nürnberg, 1795. 
PANZER (G. W. F.). Kritische Revision der Insektenfaune Deutschlands. $12^{\circ}$. Nürnberg, 1805.

PANZER (G. W. F.). Index Entomologicus.

8. Norimbergce, 1813.

PANZER (G. W. F.) \& HERRICH-SCHAEFFER (G. A.). Deutschlands Insecten. $\quad 16^{\circ}$. Nürnberg, 1793-1823. Regensburg, 1829-44.

PANZER (G. W. F.). See Schaeffer (J. C.).

PANZER (G. W. F.). See Udman (J.).

PAPPE (L.). Synopsis of the edible Fishes at the Cape of Good Hope. $\quad 8^{\circ}$. Cape Town, 1853. 2nd edition. $8^{\circ}$. Cape Town, 1866.

PARDON (G. F.). Stories about Birds.

8.. London, 1857.

PARFITT (E.). A Catalogue of the Fauna of Devon. (Zoophytes.)

8․ Exeter, 1866.

PARKER (W.K.). Monograph on the Structure and Development of the Shoulder-girdle and Sternum.

See RAy Society.

fol. London, 1866.

PARKER (W. K.). See Carpenter (W. B.).

PARKER (W. K.). See 'Challenger.'

PARKER (W. K.) \& BETTANY (G. T.). The Morphology of the Skull.

8. London, 1877.

PARNELL (R.). Fishes of the Firth of Forth.

8. Edinburgh, 1838.

PARROT (G. F.). Essai sur les Ossements fossiles des bords du lac de Burtneck, en Livonie.

$4^{\circ}$. St. Petersburg, 1836. 
PARRY (F. J. S.). Catalogus Coleopterorum Lucanoidum. 3rd edition. 80. London, 1875.

PARSONS (J.). Die natürliche Historie des Nashorns, welche von Dr. Parsons in einem Schreiben an M. Folkes angefasset; in das Deutsche übersezet von G. L. Hбтн.

$4^{\circ}$. Nürnberg, 1747.

PARSONS (J.). Philosophical Observations on the Analogy between the Propagation of Animals and that of Vegetables.

8. London, 1752.

PASCOE (F. P.). A List of the Australian Longicorns.

8.. Sydney, 1868.

PASCOE (F. P.). Zoological Classification.

2nd edition. post $8^{\circ}$. London, 1880.

PASCOE (F. P.). The Student's List of British Coleoptera. post $8^{\circ}$. London, 1882.

PASSERINI (C.). Osservazioni sulle Tarve, Ninfe, e Abitudini della Scolia flavifrons.

$4^{\circ}$. Pisa, 1840.

PASSERINI (C.). Notizie sulla Moltiplicazione in Firenze dell' uccello Americano Paroaria cucullata.

fol. Firenze, 1841.

PASSERINI (C.). Notizie relative a tre Specie d' Insetti nocivi all' Olivo.

8. Firenze, 1843.

PASTEUR (L.). Études sur la Maladie des Vers à Soie. 2 vols. $8^{\circ}$. Paris, 1870.

PATTERSON (R.). Letters on the Natural History of the Insects mentioned in Shakspeare's Plays.

8. London, 1838.

PATTERSON (R. L.). The Birds, Fishes, and Cetacea commonly frequenting Belfast Lough. $8^{\circ}$. London, 1880.

PAULLINI (C. F.). Lagographia Curiosa seu Leporis descriptio.

12. Augustce Vindelicorum, 1691. 
PAULLINI (C. F.). Lycographia seu de natura et usu Irupi libellus. 8. Francofurti, 1694.

PAULSON (O.). Zur Anatomie von Diplozoon paradoxum. 4\%. St. Petersburg, 1862.

PAULUCCI (M.). Fauna Malncologica.

$8^{\circ}$. Firenze, 1879.

PAULUCCI (M.). Faune Malacologique de l'Italie.

8. Paris, 1878.

PAULY (A.). Ueber die Wasserathmung der Limnæiden. 8. München, 1877.

PAVESI (P.). I Pesci e la Pesca nel Cantone Ticino.

8. Lugano, 1871-73.

PAVESI (P.). Materiali per una Fauna del Cantone Ticino. 8. Mitano, 1873.

PAYKULL (G. von). Monographia Staphylinorum Sueciæ. 8․ Upsaliae, 1789.

PAYKULL (G. von). Monographia Caraborum.

8. Upsalice, 1790.

PAYKULL (G. von). Monographia Curculionum Sueciæ.

8․ Upsalice, 1792.

PAYKULL (G. von). Fauna Suecica.

3 vols. $8^{\circ}$. Upsaliae, 1798-1800.

PAYKULL (G. von). Monographia Histeroidum.

8. Upsalice, 1811.

PAYNE-GALLWEY (Sir R.). The Fowler in Ireland.

8. . London, 1883.

PAYRANDEAU (B. G.). Catalogue des Annelides et des Mollusques de l'ile de Corse.

8. Paris, 1826.

PEABODY (W. O. B.). See Surveys \& Explorations: U.S., Boston. 
PEALE (R.). Account of the Skeleton of the Mammoth. $4^{\circ}$. London, 1802.

PEALE (T. R.). Lepidoptera Americana: or original figures of the Moths and Butterflies of North America.

[Imperfect.]

$4^{\circ}$. Philadelphia, 1833.

PECCHIOLI(V.). Osservazioni su' Topi-talpini (Arvicole $)$. $4^{\circ}$. Pisa, n. d.

PELLET (P.). Hist. Nat. du Département des Pyrénées Orientales: Entomologie. $\quad 8^{\circ}$. Perpignan, 1874-76.

PELZELN (A. von). Zur Ornithologie Brasiliens.

8.. Wien, 1871.

PELZELN (A. von). See ' Notara.'

PENNANT (T.). British Zoology. Published under the Inspection of the Cymmrodorian Society.

fol. London, 1766.

4th edition. 4 vols. $4^{\circ}$. Warrington, 1776-77. Another edition. 4 vols. $8^{\circ}$. London, 1812.

PENNANT (T.). Synopsis of Quadrupeds.

8. Chester, 1771.

PENNANT (T.). History of Quadrupeds.

2 vols. $4^{\circ}$. London, 1781.

PENNANT (T.). Genera of Birds [with Indexes].

$4^{\circ}$. London, 1781.

PENNANT (T.). Indexes of the Ornithologie of Buffon and the Planches Enluminées.

(See Preceding Title.)

$4^{\circ}$. London, 1786.

PENNANT (T.). Indian Zoology.

$4^{\circ}$. London, 1790 [1791]. 
PENNANT (T.). Aretic Zoology.

2 vols. $4^{\circ}$. London, 1784-85.

2nd edition. $4^{\circ}$. London, 1792.

PENNETIER (G.). L'Origine de la Vie.

$12^{\circ}$. Paris, 1868.

PERCHERON (A.). Monographie des Passales.

8. Paris, 1835.

PERCHERON (A.). Bibliographie entomologique.

2 vols. $8^{\circ}$. Paris, 1837.

PEREYASLAWZEFF (S.). Vorläufige Mittheilungen über die Nase der Fische. $\quad$ 8०. Zürich, 1876.

PERGER (A. R. von). Proben deutscher Thiernamen.

8. Wien, 1857.

PERRAULT (C.). Mémoires pour servir a l'histoire naturelle des Animaux. $\quad 4^{\circ}$. Amsterdam, 1758.

PERRIER (J. O. E.). Révision de la collection de Stellérides du Muséum d'Histoire naturelle de Paris.

See Museum (Paris).

8.. Paris, 1875.

PERRONCITO (E.). I Parassiti dell' nomo e degli animali utili. 8. Boloyna, Milano, Napoli, 1882.

PERRY(G.). Arcana : or the Museum of Natural History. 8. London, 1811.

PERRY (G.). Conchology, or the Natural History of Shells. fol. London, 1811.

PERSONNAT (C.). Le Ver à Soie du Chêne (Bombyx yama-mä̈). 8.. Paris, 1868.

PERTY (M.). Observationes nonnullæ in Coleoptera Indiæ orientalis.

4. Moncchii, 1831.

PERTY (M.). De Insectorum in America meridionali habitantium vitæ genere, moribus ac distributione geographica observationes nonnullæ.

fol. Monachii, 1833. 
PERTY (M.). Zur Kenntniss kleinster Lebensformen.

PERTY (M.). See Spix (J. B. de) \& Martius (C. F. P. de).

PERUGIA (A.). Elenco dei Pesci dell' Adriatico.

$4^{\circ}$. Milano, 1881.

PETAGNA (V.). Specimen Insectorum ulterioris Calabriæ. Editio nova. $4^{\circ}$. Lipsice, 1808.

PETERS (W. C. H.). Observationes ad Anatomiam Cheloniorum. $4^{\circ}$. Berolini, 1838.

PETERS (W.). Reise nach Mossambique. I. Säugethiere. IV. Flussfische. V. Insecten und Myriopoden.

$4^{\circ}$. Berlin, 1852-68.

PETERS (W.). Ueber die Chiropterengattung Mormops und Phyllostoma.

$4^{\circ}$. Berlin, 1857.

PETERS (W.). Ueber die Chiropterengattung N'yctophilus. $4^{\circ}$. Berlin, 1861.

PETERS (W.). Merkwürdige Nagethiere.

4\%. Berlin, 1861.

PETERS (W.). De Serpentum familia Uropeltaceorum.

4\%. Berolini, 1861.

PETERS(W.). Ueber Cercosaura aus Süd-America.

4\%. Berlin, 1862.

PETERS (W.). Ueber die Säugethier-Gattung Chiromys (Aye-Aye). $4^{\circ}$. Berlin, 1866.

PETERS (W.). Ueber Dinomys.

4\%. Berlin, 1873.

Peters (W.). See Lichtenstein (H.) \& Peters (W.).

PETERS (W.). See Von Der Decken, Reisen. 
PETIT DE LA SAUSSAYE (S.). Catalogue des Mollusques Testacés des Mers d'Europe.

8.. Paris, 1869.

PETIVER (J.). Musei Petiveriani Centuria Prima.

12․ London, 1695.

PETIVER (J.). Aquatilium animalium Amboinæ \&c Icones. fol. London, 1713.

PETIVER (J.). Opera omnia ad Historiam naturalem spectantia, \&c. 2 vols. fol. London, 1764.

PETRI (K. R.). Die Copulationsorgane der Plagiostomen. 8. Leipzig, 1877.

PETTERD (W. F.). A Monograph of the Land Shells of Tasmania. 8. Tasmania, 1879.

PEYER (J. C.). Merycologia, sive de Ruminantibus et Ruminatione commentarius. $4^{\circ}$. Basilece, 1685.

PEYERIMHOFF ( $H$. de). Catalogue des Lépidoptères d'Alsace. $2^{\mathrm{e}}$ édition par Macker et Fetrig.

8. Colmar, 1880-82.

PFAFF (C. H.). See Gaede (H. M.).

PFEIFFER (L.). Symbolæ ad Historiam Heliceorum.

8. Cassellis, 1841-46.

PFEIFFER (L.). Monographia Heliceorum.

$$
8 \text { vols. } 8^{\circ} \text {. Lipsice, 1847-77. }
$$

PFEIFFER (L.). Catalogue of the Phaneropneumona in the British Museum.

See Museum (British).

$12^{\circ}$. London, 1852.

See

PFEIFFER (L.). Monographia Auriculaceorum viventium. 8. Cassellis, 1856.

PFEIFFER (L.). Catalogne of Auriculida, Proserpinida, and Truncatellidce in the British Museum.

See Musedm (British).

12. London, 1857. 
PFEIFFER (L.). MIonographia Pneumonopomorum virentium. 1 vol. \& 3 Supplem. $8^{\circ}$. Cassellis, 1858-75.

PFEIFFER (L.). See Novttates Conchologicze.

PFEIfFER (L.). See Periodicals: Cassel.

PFEIFFER (L.) \& CLESSIN (S.). Nomenclator Heliceorum. $8^{\circ}$. Cassel, 1878.

PFLÜGER (E. T. W.). See Periodicals : Bonn.

PHILIBERT (H.). Aristotelis Philosophia Zoologica.

8. Parisiis, 1865.

PHILIPPI (R. A.). Orthoptera Berolinensia.

4․ Berolini, 1830.

PHILIPPI (R. A.). Enumeratio Molluscorum.

2 vols. $4^{\circ}$. Berolini, 1836-44.

PHILIPPI (R. A.). Abbildungen und Beschreibungen neuer Conchylien. Vcl. I. $4^{\circ}$. Cassel, 1845.

PHILIPPI (R. A.). Handbuch der Conchyliologie und Malacozoologie. $\quad 8^{\circ}$. Halle, 1853.

PHILIPS (J.). Imperial Library Atlas. imp. fol. London, 1883.

PHILOCHELIDON. Observations on the brumal retreat of the Swallow. By Philochelidon.

2nd edition. $8^{\circ}$. London, 1808.

'PHysicienne.' See ' Uranie' \& ' Physicienne.'

PIAGET (E.). Les Pédiculines.

2 vols. $4^{\circ}$. Leide, 1880.

PIANA (G. P.). Di una nuova specie di Tenia del Gallo domestico (Tcenia botrioplitis), e di un nuovo Cisticerco delle Lumachelle terrestri (Cysticercus botrioptitis).

$4^{\circ}$. Bologna, 1881. 
PIANA (G. P.). Le Cercarie nei Molluschi.

8. Milano, 1882.

PICCIOLI (F.). Catalogo della Collezione di Insetti Italiani del R. Museo di Firenze. Coleotteri, ser. 1 \& 2.

8. Firenze, 1876-79.

PICKARD-CAMBRIDGE (O.). See Cambrtdge (O. P.).

PICTET (A. E.). Synopsis des Neuroptères d'Espagne.

8.. Genève, 1865.

PIETREJENT (C. A.). Les Chevaux dans les Temps Préhistoriques et Historiques. $\quad 8^{\circ}$. Paris, 1883.

PIGEONS, a Treatise on Domestic. [Anon.]

8. London, 1765.

PILLER (M.) \& MITTERPACHER (L.). Iter per Poseganam Sclaroniæ Provinciam. $4^{\circ}$. Buclce, 1783.

PINI (N.). Molluschi terrestri e d' acqua dolce viventi nel Territorio di Esino.

$8^{\circ}$. Milano, 1876.

PIRAZZOLI (0.). Coleopteri Italici genus novum, Leptomastix.

8. Forocomelii, 1855.

PIRAZZOLI (O.). Coleotteri Italiani. $\quad 8^{\circ}$. Imola, 1882.

PITFIELD (A.). Memoirs for a Natural History of Animals, containing the anatomical descriptions of several creatures dissected.

4\%. London, 1788.

PLANCUS (J.). De Conchis minus notis liber.

$4^{\circ}$. Venetiis, I739.

PLAYFAIR (R. L.) \& GÜNTHER (A.). The Fishes of Zanzibar.

$4^{\circ}$. London, 1866.

PLINIUS SECUNDUS (Caius). Naturalis Historiæ libri xxxrii. 1st edition. fol. Venetiis, 1469.

PLINIUS. See MERreM (B.). 
PLUCAR (E.). Der Fischplaz zu Triest.

8. $\quad$ Triest, 1846.

PODWISOTZKY (V.). Anatomische Untersuchungen über die Zungendrüsen des Menschen und der Säugethiere.

8. Dorpat, 1878.

POEY (F.). Memorias sobre la Historia Natural de la Isla de Cuba. 8. Habana, 1851-58.

POEY (Ph.). Centurie de Lépidoptères de l'Ile de Cuba.

8. Paris, 1832.

POLI(J. X.) \& DELLE CHIAJE (C.). Testacea utriusque Siciliæ eorumque Historia et Anatome tabulis æneis illustrata. Cum additamentis S. Delue ChiaJe.

3 vols. \& Atlas. fol. Parma, 1791-1826.

POLLEN (F. P. L.) \& VAN DANI(D. C.). Recherches sur la Faune de Madagascar et de ses dépendances.

$4^{\circ}$. Leycle, 1868-77.

POLLOCK (F.). See Fismeries : London. International Fisheries Exhibition, 1883.

POOLE (W. F.) \& FLETCHER (W. J.). An Index to Periodical Literature. $4^{\circ} . \quad B o s t o n$ \& London, 1882.

POSSELT (C. F.). Beyträge zur-Anatomie der Insekten. $4^{\circ}$. Tübingen, 1804.

POTIEZ (V. L. V.) \& MICHAUD (A. L. G.). Galerie des Mollusques du Muséum de Douai.

2 vols. \& Atlas. $8^{\circ} . \quad$ Paris, 1838-44.

POUCHET (F. A.). Anatomie et Physiologie des Mollusques. $\quad 4^{\circ}$. Paris, 1842.

POUCHET (G.). Mémoires sur lo Grand Fourmilier (Myrmecophaga jubata). 4. Paris, 1874.

POURTALES (L. F. de). Deep-Sea Corals. $4^{\circ}$. Cambridge, U.S., 1871.

See Museum: Harvard College. Memoirs. 
Pourtales (L. F. de). See Agassiz (A.) \& Pourtales (I. F. de).

POWER (J.). See Harvey (J. R.).

PRADA (T.). Sui Curculioniti dell' agro Pavese.

$8^{\circ}$. Milano, 1860.

PRAUN (S. von). Abbildung und Beschreibung Europäischer Schmetterlinge. (Nicrolepidoptera.)

4. Nürnberg, 1869.

PRAUT (S. ron). Abbildung und Beschreibung Europäischer Schmetterlinge. $\quad 4^{\circ}$. Nürnberg, 1858-80.

PRAUN (S. ron). Abbildung und Beschreibung Europäischer Schmetterlingsraupen. $4^{\circ}$. Nümberg, 1874-75.

PRAZMIOTSKI (A.). Tntersuchungen über die Entwickelungsgeschichte und Fermentwirkung einiger Bacterien-Arten.

8․ Leipzig, 1880.

PRECHTL (J. J.). Untersuchungen ïber den Flug der Vögel. $8^{\circ}$. Wien, 1846.

PRELLER (C. H.). Beiträge zur einem natürlichen System der Coleopteren. $\quad 8^{\circ} . \quad$ Jena, 1861.

PREVOST (F.). See 'VENUs.'

PREYSSLER (J. D.). Verzeichniss Böhmischer Insekten (erstes Hundert). $\quad 4^{\circ}$. Prag, 1790.

PRICHARD (J. C.). Researches into the Physical History of Mankind.

3rd edition. 5 vols. London, 1836-47. Another edition. 5 vols. London, 1837-51.

PRICHARD (J. C.). The Natural History of Man. 4th edition by E. Norris. 2 vols. $8^{\circ}$. London, 1855.

PRICHARD (J. C.). Six Ethnographical Maps illustrative of "The Natural History of Man."

2nd edition. fol. London, n. d. 
PRITCHARD (A.). A History of Infusoria, living and fossil. $8^{\circ}$. London, 1841.

PRITCHARD (A.). Notes on Natural History.

$12^{\circ}$. London, 1844 .

PRITCHARD (A.). A History of Inf usorial Animalcules, living and fossil.

$8^{\circ}$. London, 1852.

PRUNNER (L. de). Lepidoptera Pedemontana.

8․ Aug. Taurinorum, 1798.

PUCHERAN (J.). Des Caractères Zoologiques des Mammifères. $4^{\circ}$. Paris, 1860 .

PULTENEY (R.). A general View of the writings of Linnæus. Second edition, by W. G. Maton.

$4^{\circ}$. London, 1805.

PUTON (E.). Essai sur les Mollusques terrestres et fluviatiles des Vosges.

$8^{\circ}$. Epinal, 1847.

PUTON (Dr.). Synopsis des Hémiptères-Hétéroptères do France. Parts I.-II. $8^{\circ}$. Paris, 1878-79. Part III. $8^{\circ}$. Remiremont, 1880.

Q.

QUARITCH (B.). A general Catalogue of Books. thick $8^{\circ}$. London, 1880.

QUATREFAGES (A. de). Histoire naturelle des Annélés marins et d'eau douce. Annélides et Géphyriens.

2 vols. $8^{\circ}$. Paris, 1865.

QUATREFAGES (A. de). Hommes fossiles et Hommes saurages.

$8^{\circ}$. Paris, 1884 .

QUATREFAGES (A. de) \& HAMY (E. T.). Crania Ethnica. Les Crânes des Races Humaines.

2 vols. $4^{\circ}$. Paris, 1882. 
QUatrefages (A. de). See Mrlne-Edwards (H.).

QUENSEL (C.). Utkast till Elefantens Naturalhistoria. (Bound with Srensk Zoologi.) 8. Stockholm, 1804.

QUENSEL (C.). See Stensi Zoologi.

QUEKETT (J.). Lectures on Histology.

2 vols. $8^{\circ}$. London, 1852-54.

QUEKETT (J.). See Mrdsedx: College of Surgeons.

QUOY (J. R. C.) \& GaIMIARD (J. P.). See 'Uranie' \& 'Physicienne.'

R.

RADDE (G.). Thierleben am Faulen Meere.

8.. Moscau, 1855.

RADDE (G.). Reisen im Süden von Ost-Sibirion.

$4^{\circ}$. St. Petersburg, 1862.

RAFINESQUE (C. S.). A Life of Travels and Researches in North America and South Europe.

16º. Philadelphia, 1836.

RAFINESQUE SCHMIATZ (C. S.). Caratteri di alcuni nuovi generi e nuove specie di animali e piante della Sicilia. $\quad 8^{\circ}$. Palermo, 1810.

RAFINESQUE SCHMALTZ (C. S.). Indice d' Ittiologia Siciliana. 8. Messina, 1810.

RAFINESQUE SCHMALTZ (C. S.). Précis des découvertes et travaux somiologiques. $16^{\circ}$. Palermo, 1814.

RAINEY (G.). On the Mode of Formation of Shells of Animals, of Bone, \&c.

$8^{\circ}$. London, 1858. 
RAMANN (G.). Die Schmetterlinge Deutschlands. $4^{\circ}$. Arnstadt.

RAMBAUD (A.) \& RENAULT (C.). Origine et développement des Os. Texte, $8^{\circ}$. Atlas, fol. Paris, 1864.

RAMBUR (P.). Faune Entomologique de l'Andalousie. 8. Paris, 1842 .

RAMBUR (P.). Histoire naturelle des Insectes (Neuroptères). 8.. Paris, 1842.

RAMBUR (P.). Catalogue Systématique des Lépidoptères de l'Andalousie.

8․ Paris, 1858-66.

RAMDOHR (K. A.). Abhandlung über die Verdauungswerkzeuge der Insecten.

$4^{\circ}$. Halle, 1811.

RAMON DE LA SAGRA. See Moseum (British).

RAMSAY (R. G. W.). See Twewddale (Marquis of).

RANG (P. C. A. L.) \& SOULEYET. Histoire naturelle des Mollusques Ptéropodes.

$4^{\circ}$. Paris, 1852.

RANG (S. A.). Manuel de l'histoire naturelle des Mollusques.

$12^{\circ}$. Paris, 1829.

RANKE(J.). Beiträge zur physischen Anthropologie der Bayern. $4^{\circ}$. München, 1883.

RANZANI (C.). Elementi di Zoologia.

13 vols. $8^{\circ}$. Bologna, 1819-26.

RANZANI (C.). Memorie di Storia Naturale.

$4^{\circ}$. Bologna, 1820 .

RANZANI (C.). Descrizione di un Serpente il quale appartiene ad una nuova specio del genere Calamaria di Boie. $4^{\circ}$. Firenze, 1835.

RAPP (W. von). Anzeige der Eröffnung des neuerbauten anatomischen Theaters der Universität Tübingen.

fol. Tübingen, 1836. 
RAPP (W. von). Anatomische Untersuchung des Orycteropus capensis.

$4^{\circ}$. Stuttgart, 1837.

RAPP (W. von). Die Cetaceen, zoologisch-anatomisch dargestellt. 8.. Stuttgart \& Tübingen, 1837.

RAPP (W. von). Anatomische Untersuchungen über die Edentaten. 40. Tübingen, 1843. Another edition. $4^{\circ}$. Tübingen, 1852.

RAPP (W. von). Die Fische des Bodensees.

Text, 8․ Plates, obl. fol. Stuttgart, 1854.

RAPP (T. L.) \& BENGEL (E.). Osteologie des indischen Krokodils. $8^{\circ}$. Tübingen, 1834.

RAPP (W. L.) \& DUTTENHOFER (F. M.). Ueber die zusammengesezten Mägen rerschiedener Thiere.

4\%. Tübingen, 1832.

RAPP (W. L.) \& ELSAESSER (C. L.). Disquisitiones Zoologico-physiologicæ. 4. Tubingce, 1830.

RAPP (W.) \& J ÄGER (A.). See J̈̈GER (A.).

RASCH (H.). Delphinus leucopleurus, nova species.

40. Christianice, 1843.

RASPAIL (F: V.). Histoire Naturelle de la Santé et de la Maladie chez les végétaux, les animaux, et chez l'homme. 3 vols. Paris \& Bruxelles, 1860.

RATH (J.). De Geographica Avium Distributione.

8. Herbipoli, 1831.

RATHBUN (R.). See Fisheries : London. International Fisheries Exhibition, 1883.

RATHKE (H.). Beiträge zur Geschichte der Thierwelt. 4 vols, in $3.4^{\circ}$. Danzig \& Halle, 1820-27.

RATHKE (H.). Untersuchungen über den Kiemenapparat und das Zungenbein der Wirbelthiere.

4. Riga \& Dorpat, 1832. 
RATHKE (H.). De Bopyro et Nereide, commentationes anatomico-physiologicæ duæ. $4^{\circ}$. Rige \& Dorpati, 1837.

RATHKE (H.). Zur Fauna der Krym.

4. St. Petersburg, 1837.

RATHKE (H.). Entwickelungsgeschichte der Natter (Coluber natrix). $4^{\circ}$. Königsberg, 1839.

RATHKE (H.). Bemerkungen über die Entwickelung des Schädels der Wirbelthiere.

4. Königsberg, 1839.

RATHKE (H.). Bemerkungen über den Bau des $A m$ phioxus lanceolatus.

$4^{\circ}$. Königsberg, 1841.

RATHKE (H.). Beiträge zur Vergleichenden Anatomie und Physiologie.

$4^{\circ}$. Danzig, 1842.

RATHKE (H.). Ueber die Entwickelung der Schildkröten.

4\%. Braunschweig, 1848.

RATHKE (H.). Ueber den Bau und die Entwickelung des Brustbeins der Saurier.

$4^{\circ}$. Königsberg, 1853.

RATHKE(H.). Entwickelungsgeschichte der Wirbelthiere. Vorwort von A. KöLLIKER. 8.. Leipzig, 1861.

RATHKE (H.). Vorträge zur vergleichenden Anatomie der Wirbelthiere. Vorwort von C. Gegendadr.

8.. Leipzig, 1862.

RATHKE (H.). See Leuckart (R.).

RATZEBURG (J. T. C.). Die Forst-Insecten.

3 vols. $4^{\circ}$. Berlin, 1839-44.

RATZEBURG (J. T. C.). Die Ichneumonen der ForstInsecten. 3 vols. $4^{\circ}$. Berlin, 1844-48.

RATZEBURG (J. T. C.). Die Waldverderber und ihre Feinde. 5th edition. $8^{\circ}$. Berlin, 1860.

RAUBER (A.). Ueber die Stellung des Hühnchens in Entwicklungsplan. 4․ Leipzig, 1876. 
RAUBER (A.). Primitirstreifen und Neurula der Wirbelthiere, in normaler und pathologischer Beziehung.

8. Leipzig, 1877.

RÄ̈BER (H.). See Kossiraxa (R.).

RAUTENFELD (E. von). Morphologische Untersuchungen über das Skelet der hinteren Gliedmassen von Ganoiden und Teleostiern.

8. Dorpat, 1882.

RAY (J.). Synopsis Methodica Animalium Quadrupedum et Serpentini Generis.

8. Londini, 1693.

RAY (J.). Historia Insectorum. $\quad 4^{\circ}$. Londini, 1710.

RAY (J.). Synopsis methodica Arium et Piscium.

$8^{\circ}$. Londini, 1713.

RAY (J.). See Salerate (F.).

RAY (J.). See Willdghbi (F.).

RAY SOCIETY. Publications.

$8^{\circ} \&$ fol. London, 1845-79.

I. The Octavo Series.

AgAssiz (L.). Bibliographia Zoologiæ et Geologiæ. Edited by H. E. StrickLand. 4 vols. 1848.

BAIRd (W.). The Natural History of the British Entomostraca.

1850 .

Boyaparte (C. L.). Reports on the Progress of Zoology and Botany for 1841-42.

1845 .

Bowerbaxk (J.S.). Monograph of the British Spongiada. 4 rols. 1864-8\%.

Vol. IV. additions by A. M. Norman.

Brady (G. S.). A Monograph of the Free and Semiparasitic Copepoda of the British Islands.

3 vols. 1878.

Bucktox (G. B.). Monograph of the British Aphides. 2 vols. $1876-79$.

Busk (G.), Tulk (А.), \& Haliday (А. H.). Reports on Zoology for 1843, 1844.

(Translated from the German.)

1847. 
DaRWIN (C.). A Monograph of the Cirripedia.

2 vols. 1851-54.

Douglas (J. W.) \& Scotr (J.). British Hemiptera. 1865.

LaNkester (E.). Memorials of John Ray. 1846.

Lшввоск (J.). Monograph of the Collembola and Thysanura.

1873.

Owen (R.). On the Archetype and Homologies of the Vertebrate Skeleton.

1848.

Steenstrup (J. J. L.). On the Alternation of Generations, translated by G. Busk.

1845.

\section{The Folio Series.}

Alder (J.) \& Harcock (A.). A Monograph of the British Nudibranchiate Mollusca.

1845-55.

Allman (G. J.). A Monograph of the Freshwater Polyzoa.

1856.

Allman (G. J.). A Monograph of the Gymnoblastic or Tubularian Hydroids.

1871.

Blackwall (J.). A History of the Spiders of Great Britain and Ireland. 1860-64.

Carpenter (W. B.), Parker (W. K.), \& Jones (T. R.). Introduction to the Study of the Foraminifera. 1862.

Flower (W. H.). Recent Memoirs on the Cetacea, by Proff. Eschricht, Reinhardt, and Lilljeborg. 1866.

Forbes (E.). A Monograph of the British Nakedeyed Medusæ.

1848.

GüNther (A.). The Reptiles of British India. 1864.

Huxtey (T. H.). The Oceanic Hydrozoa. 1859.

M'Intosh (W. C.). A Monograph of the British Annelids. (The Nemerteans.) 1873-74.

Nitzsch's Pterylography, translated from the German. Edited by P. L. Sclater.

1867.

PARKER (W. K.). A Monograph on the Structure and Development of the Shoulder-girdle and Sternum in the Vertebrata.

1868.

Williamson (W. C.). On the Recent Foraminifera of Great Britain.

1858. 
REAUMUR (R. A. F. de). The Natural History of Bees. Translated from the French. $\quad 8^{\circ}$. London, 1774.

REAUMUR (R. A.F. de). Mémoires pour servir à l'histoire des Insectes. 6 vols. $4^{\circ}$. Paris, 1834-42.

REAUMUR (R. A. F. de). See VALLOT (J. N.).

REDI (F.). Osservazioni de F. R. intorno agli animali viventi che si trovano negli animali virenti.

$4^{\circ}$. Firenze, 1684.

REDI (F.). Osservazioni intorno alle Vipere. $4^{\circ}$. Firenze, 1686.

REDI (F.). Esperienze intorno alla Generazione degl' Insetti. $4^{\circ}$. Firenze, 1674. Another edition. $4^{\circ}$. Firenze, 1688.

REDI (F.). Consulti Medici.

2 rols. in 1. $4^{\circ} . \quad$ Napoli, 1740.

REDI (F.). Opere. 5 rols. in $2 . \quad 4^{\circ} . \quad$ Napoli, 1741.

REDTENBACHER (L.). Fauna Austriaca. Die Käfer.

8. Wien, 1849.

REDTENBACHER (L.). Fauna Austriaca. 2nd edition. $8^{\circ}$. Wien, 1858. 3rd edition. $8^{\circ}$. Wien, 1872-74.

REDTENBACHER (L.). See ' Notara.'

REED (E. C.). Catalogo de los Insectos Chilenos.

So. Santiago, 1874.

REEVE (H.). An Essay on the Torpidity of Animals.

80. London, 1809.

REEVE (L. A.). Conchologia Systematica.

2 vols. $4^{\circ}$. London, 1841-42.

REEVE (L. A.). Elements of Conchology.

$8^{\circ}$. London, 1846. Another edition. 2 vols. $8^{\circ}$. London, 1860. 
REEVE (L. A.). Conchologia Iconica.

20 vols. $4^{\circ}$. London, 1843-78.

REEVE (L. A.). See Catlow (A.).

REEVE (L. A.). See 'SAMraRang.'

REEVES (J.). Original Drawings and Plates of Mammalia, Birds, Reptiles, Fishes, Insects, Mollusca, and Crustacea.

6 vols. imp. fol.

REGAZZONI (J.). Notizie dei Pesci delle Provincie di Como. 2nd edition. $12^{\circ}$. Como, 1864.

REGAZZONI (J.). L' uomo Preistorico nella Provincia di Como. 4\%. Milano, 1878.

REGGER (J.). See Krein (J. T.).

REICH (G. C.). Mantissæ Insectorum.

Fasc. I. $8^{\circ}$. Noribergce, 1797.

REICHENAU (W. von). Die Abstammung der Vögel und Vogelleben in den oberbairischen Voralpen.

8. Mainz, 1876.

REICHENAU (W. von). Die Nester und Eier der Vögel. (Darwinistische Schriften, No. 9.) 8. Leipzig, 1880.

REICHENBACH (H. G. L.). Monographia Pselaphorum, adjuncto socio Gustar Kunze. $\quad$ 80. Lipsice, 1816.

REICHENBACH (H. G. L.). Synopsis Mammalium.

$8^{\circ}$. Lipsice, 1845.

REICHENBACH (H. G. L.). Handbuch der speciellen Ornithologie. roy. $8^{\circ}$. Dresden, 1852.

REICHENBACH (H. G. L.). Die vollständigste Naturgeschichte der Vögel.

4 vols. text, 13 vols. plates. $4^{\circ}$. Dresden \& Leipzig, 1836-55.

REICHENBACH (L.). See Meyer (A. B.). 
REICHENHEIM (M.). Ueber das Rückenmark und den electrischen Lappen von Torpedo. $\quad 4^{\circ}$. Berlin, 1878.

REICHENOW (A.). Die Vögel der Zoologischen Gärten. 8.. Leipzig, 1882.

REICHENOW (A.). Vogelbilder aus fernen Zonen. Abbildungen und Beschreibungen der Papageien.

fol. Kassel, 1883.

REICHENOW (A.). See Pertodicals : Bertin.

REICHERT (C. B.). Vergleichende Entwickelungsgeschichte des Kopfes der nackten Amphibien.

$4^{\circ}$. Königsberg, 1838.

REICHERT (C. B.). Das Entwickelungsleben im Wirbelthier-Reich.

$4^{\circ}$. Berlin, 1840.

REICHERT (C. B.). Beiträge zur Kenntniss des Zustandes der heutigen Entwickelungs-Geschichte.

8. Berlin, 1843.

REICHERT (C. B.). Die monogene Fortpflanzung.

4\%. Dorpat, 1852.

REICHert (C. B.). See Pertodtcals: Berlin and Letpzig. Archiv für Anatomie und Physiologie.

REIDER (J. E. von) \& HAHN (C. W.). Fauna Boica, oder gemeinnützige Naturgeschichte der Thiere Bayerns.

4 vols. $8^{\circ}$. Nürnberg, 1830-35.

REIFF (C. P.). See Dictionaries : Russian.

REIL (J. C.). See Pertodicals: Halle.

REIMIANN (C. G. E.). Spicilegium Observationum anatomicarum de Hyæna. $4^{\circ}$. Berolini, 1811.

REINHARDT (J.). Natur-historisk Dagbog. (Invertebrata.) $4^{\circ}$. MS. 1808.

REINHARDT (J.). Quinque Mumias bestiarum .ÆEgyptiacas describendo prolusit J. R.

$4^{\circ}$. Havnice, 1824. 
REINHARDT (J.). Ichthyologiske Bidrag til den Grönlandske Fauna. 40. Kjöbenhavn, 1837.

REINHARDT (J.). Naturhistoriske Bidrag til en Beskrivelse af Grönland.

8.. Kjöbenhavn, 1857.

REINHARDT (J.). Mephitis vestermanni.

4․ Kjöbenhavn, 1857.

REINHARDT (J.). Falkejagten.

8. Kjöbenhavn [1858?].

REINHARDT (J.). Bidrag til Kundskab om Fuglefaunaen i Brasiliens Campos. 8. Kjöbenhavn, 1870.

REINHARDT (J.). See Eschricht (D. F.).

REINHARDT (J.). See RAy Societr.

REISINGER (J.). Specimen Ichthyologiæ sistens pisces aquarum dulcium Hungariæ. $\quad$ 8․ Budce, 1830.

REISSNER (E.). Beiträge zur Kenntniss der Haare des Menschen und der Säugethicre. $\quad 8^{\circ}$. Breslau, 1854.

REISSNER (E.). Der Bau des centralen Nervensystemes der ungeschwänzten Batrachier.

Text and Atlas. $4^{\circ}$. Dorpat, 1864.

RENARD (A.). See ' Challenger.'

RENARD (L.). Histoire Naturelle des plus rares Curiosités de la mer des Indes. Poissons, Ecrivisses, et Crabes ..... des Moluques.

2 vols. in 1. fol. Amsterdam, 1718.

RENARD (L.) \& VOSMAER (A.). Poissons, Ecrevisses, et Crabes des Isles Moluques, et des Terres Australes.

fol. Amsterdam, 1754.

RENGGER (A.). Reise nach Paraguay in den Jahren 1818-26.

8. Aarau, 1835. 
RENGGER (J. R.). Untersuchungen über die thierische Haushaltung der Insecten. $\quad 8^{\circ}$. Tübingen, 1817.

RENIER (S.). See Meneghtar (G.).

RENNIE (J.). Insect Architecture.

New edition. 8०. London, 18.57.

RENNIE (J.). See Montagd (G.).

RENOOZ (C. M.). L'Origine des Animaux.

Vol. I. parts 1,2. $8^{\circ}$. Paris, 1883.

REPORTS.

Asiatic Societr, Calcutta. Reports of the Curator of the Society's Museum, Zoological Department.

See Museum (Calcutta).

Birminghan. Report of the Free Library Committee, 1871.

$8^{\circ}$. Birmingham, 1871.

Corswall. Reports of the Royal Institution of Cornwall.

Seo Periodicals: Truro.

Irelaxd. Reports of the Royal Zoological Society of Ireland.

See Periodicals: Dublin.

Lovdon. Reports of the Council of the Zoological Society.

See Periodicals : London.

Montrose. Reports of the Directors of the Natural History Society of Montrose.

See Periodicals: Montrose.

United States :-

Albany. Reports on the Noxious, Beneficial, and other Insects of the State of New York. By A. Eттсн.

8. Albany, 1856-65.

Albany. Tenth Annual Report of the Regents of the University of the State of New York.

$$
\text { 8०. Albany, } 185 \% \text {. }
$$

Boston. Annual Reports of the Trustees of the Museum of Comparative Zoology, at Harvard College, in Cambridge.

$$
\text { 8. Boston, 1862-64, 1868-69, 1873-76. }
$$

New York. The Third and Fourth Annual Reports of the American Museum of Natural History.

$8^{\circ}$. New York, 1872. 
RE PORTS, continued.

Toronto. Annual Reports of the Entomological Society of the Province of Ontario.

$8^{\circ}$. Toronto, 1872-77.

Washington. Annual Reports of the Board of Regents of the Smithsonian Institution to the Senate and House of Representatives.

$8^{\circ}$. Washington, 1849-53.

Washington. Reports on the Noxious, Beneficial, and other Insects of the State of Missouri. By C. V. RILey. I.-IX. with Suppl.

8. Jefferson City \& Washington, 1869-81.

Washivgton. First Annual Report of the U. S. Entomological Commission 1877, relating to the RockyMountain Locust. By C. V. Riley, A. S. Packard, and C. Thomas.

$8^{\circ}$. Washington, 1878.

Washington. Second Report of the U.S. Entomological Commission 1878-79, relating to the Rocky Mountain Locust, and the Western Cricket. By C. V. Riley, A. S. Packard, and C. Thomas.

$8^{\circ}$. Washington, 1880.

Washington. Report of the Entomologist of the U.S. Department of Agriculture for the years 187981. By J. H. Comstock.

$8^{\circ}$. Washington, 1880-82.

Washington. Report of the Entomologist, Department of Agriculture, 1881. By C. V. RILEY.

8. Washington, 1882.

REQUIEN (E.). Catalogue des Coquilles de l'île de Corse. 8. Avignon, 1848.

RESTE (B. de). Histoire des Pêches, des Décourertes, et des Etablissemens des Hollandois.

3 vols. $8^{\circ}$. Paris [1801].

RETZIUS (A. J.). Fauna Sueciæ a C. Linné inchoata.

Pars I. $\quad 8^{\circ}$. Lipsice, 1800.

RETZIUS (A. J.). See Geer (C. de).

RETZIUS (G.). Finska Kranier, jämte några natur- och Literatur-studier inom andra områden af Finsk Antropologi.

$4^{\circ}$. Stockholm, 1878. 
RETZIUS (G.). Biologische Untersuchungen.

$4^{\circ}$. Stockholm, 1881.

RetZiUS (G.). See Periodicals : Stocrholdr.

REUSS (A.). Zoologische Miscellen. Reptilien. $4^{\circ}$. Frankfurt am MI., 1833.

RETOIL (G.). Faune et Flore des Pays Comalis (Afrique orientale).

8. Paris, 1882.

REY (E.). Synonymik der Europäischen Brutrögel und Gäste. 8․ Halle, 1872.

RICCARDI (P.). Studi intorno ad alcuni crani Araucanos e Pampas. $4^{\circ}$. Roma, 1879.

RICHARDSON (C.). See Dictionaries: English.

RICHARDSON (Sir J.). Fauna Boreali-Americana.

4 rols. in 3. $4^{\circ}$. London, 1829-37.

RICHARDSON (Sir J.). Icones Piscinm, or Plates of rare Fishes.

$4^{\circ}$. London, 1843.

RICHARDSON (Sir J.). Report on the Iehthyology of the Seas of China and Japan. $\quad 8^{\circ}$. London, 1846.

RICHARDSON (Sir J.). The Iuseum of Natural History (Fishes).

$4^{\circ}$. London, 1860-62.

RICHARDSON (Sir J.). See 'Blossoar.'

RICHARDSON (Sir J.). See 'Erebes' \& 'Terror.'

RICHARDSON (Sir J.). See ' HeraLd.'

RICHARDSON (Sir J.). See Mr'Irrattr (J.).

RICHARDSON (Sir J.). See 'Samarang.'

RICHARDSON (Sir J.). See YARRell (W.). 
RICHIARDI (S.). Monografia della famiglia dei Pennatularii. 8. Bologna, 1869.

RICHTER (J. G. O.). Ichthyo-theologie, oder, Vernunftund Schriftmässiger Versuch die Menschen aus Betrachtung der Fische zur Bewunderung, Ehrfurcht und Liebe ihres grossen, liebreichen und allein weisen Schöpfers zu führen.

8. Leipzig, 1754.

RICHTER (J. A.). Systematisches Verzeichniss der Schmetterlinge des Kronlandes Salzburg (Macrolepidoptera). 8. Salzburg, n. d.

RICHTERS (F.). See MöвIUs (K.).

RIDGWAY (R.). See BAIRd (S. F.).

RIDINGER (J. E.). Contemplatio Ferarum Bestiarum ; obl. fol. Aug. Vindelicorum, 1736. et Divertissemens des grands Seigneurs. obl. fol. 1729-36. (2 vols. in 1.)

RIEGELS (N. D.). Scrutatio anatomico-philosophica de Erinaceo. $12^{\circ}$. Havnice, 1799.

RIESENTHAL (O. v.). Die Raubvögel Deutschlands und des angrenzenden Mitteleuropas.

Text, 8․ Atlas, $4^{\circ}$. Cassel, 1876.

RIESS (J. A.). Der Bau der Kiemenblätter bei den Knochenfischen. 8. Bonn, 1881.

RIGACCI (G.). Necrologia di Giovanni Rigacci.

8․ Roma, 1871.

RILEY (C. V.). On the Rocky Mountain Locust.

See SURvers \& Explorations: U.S., Washington.

RILEY (C. V.). Reports on the Noxious, Beneficial, and other Insects of the State of Missouri.

See Reports : U.S., Washington.

RIMBAUD (J. B. A.). L'Industrie des Eaux Salées.

8. Paris, 1869. 
RISSO (A.). Ichthyologie de Nice. $\quad 8^{\circ}$. Paris, 1810.

RISSO (A.). Histoire naturelle des Crustacés des environs de Nice.

8.. Paris, 1816.

RISSO (A.). Histoire Naturelle des Principales Productions de l'Europe Méridionale. 5 vols. $\quad 8^{\circ}$. Paris, 1826.

RIVIĖRE (A.). Note paléontologique, ou description de quelques espèces animales fossiles. $\quad 4^{\circ}$. Paris, 1837.

RIVIĖRE (A.). Note sur un énorme fossile trouvé dans la Louisiane. 8.. Paris, 1837.

RIVIERE (E.). De l'antiquité de l'homme dans les Alpes Naritimes. Livraisons I.-VIII. 4\%. Paris, 1878-79.

ROBERT (L. P.). See Harting (J. E.).

ROBERTS (?). See Dampier (W.).

ROBERTS (S. R.). Catalogues of Porcellanictce and Amphiperasida. 8०. Philadelphia, 1869.

ROBIN (C.). Mémoire sur le développement embryogénique des Hirundinées. $\quad 4^{\circ} \quad$ Paris, 1875.

ROBIN (C.). See Periodicals: Paris.

ROBINEAU-DESVOIDY (J. B.). Recherches sur l'Organisation vertébrale des Crustacés, des Arachnides et des Insectes. $8^{\circ}$. Paris, 1828.

ROBINEAU-DESVOIDY (J. B.). Diptères des Environs de Paris. Sous direction de H. Moncead.

2 vols. $8^{\circ}$. Paris, 1863.

ROBINSON (P.). See FisherIes: London. International Fisheries Exhibition, 1883.

ROCCA (l'Abbé della). Traité complet sur les Abeilles. 3 vols. $8^{\circ}$. Paris, 1790. 
ROCHEBRUNE (A. T. de). Faune de la Sénégambie.

Mammifères.

8. Paris, 1883.

Poissons.

8. $\quad$ Paris \& Bordeaux, 1883.

RODD (E. H.). The Birds of Cornwall and the Scilly Islands. Edited by J. E. Harting. $8^{\circ}$. London, 1880.

ROEBUCK (W. D.). See Clarke (W. E.).

ROEMER (F.). Monographie der fossilen Crinoidenfamilie der Blastoideen. 8. Berlin, 1852.

ROEMER (J. J.). Genera Insectorum Linnæi et Fabricii iconibus illustratata. 4\%. Vitoduri Helvet., 1789.

ROGER (J.). Coleoptera: Verzeichniss der bisher in Oberschlesien aufgefundenen Käferarten.

8. Breslau, 1856.

ROGER (0.). Das Flügelgeäder der Käfer.

$8^{\circ}$. Ertangen, 1875.

ROLLESTON (G.). Forms of Animal Life, being outlines of Zoological Classification based upon Anatomical Investigation.

$8^{\circ}$. Oxford, 1870.

ROLLETT (A.). See Periodicals : Leipzig.

ROLPH (W. H.). Untersuchungen über den Bau des Amphioxus lanceolatus.

8. Leipzig, 1876.

RÖMER (E.). Kritisch Untersuchung der Arten des Molluskengeschlechts Venus bei Linné und Gmelin.

$8^{\circ}$. Cassel, 1857-58.

RÖMER (E.). See Notitates Conchologic.s.

RONALDS (A.). The Fly-fisher's Entomology.

New edition. $8^{\circ}$. London, 1883.

RONDANI (A. C.). Dipterologiæ Italicæ Prodromus. 7 vols. in 4. $8^{\circ}$. Parmce, 1856-71. 
RONDELET (G.). Libri de Piscibus Marinis.

fol. Lugdini, 1554.

RONDELET (G.). See Boussuet (F.).

RÖSEL VON ROSENHOF (A. J.). Der monatlich-herausgegebenen Insecten-Belustigungen.... Theil.

4 vols. $4^{\circ}$. Nürnberg, 1746-61.

RÖSEL VON ROSENHOF (A. J.). Historia naturalis Ranarum nostratium, cum præfatione viri A. v. HaLler. Die nat. Hist. der Frösche.

fol. Nürnberg, 1758.

ROSENHAUER (W. G.). Beiträge zur Insekten-Fauna Europas.

8. Erlangen, 1847.

ROSENHAUER (W. G.). Die Thiere Andalusiens.

8. Erlangen, 1856.

ROSENTHAL (F.). Einige naturhistorische Bemerkungen über die Walle.

$4^{\circ}$. Greifswald, 1827.

ROSENTHAL (F.). Ichthyotomische Tafeln.

2nd edition. fol. Berlin, 1839.

ROSENTHAL (F.) \& HORNSCHUCH (F.). Epistola de Balænopteris. $4^{\circ}$. Gryphice, 1825.

ROSENTHAL (J.). See Hermana (L.). Handbuch der Physiologie.

ROSER (R.). Naturhistorische und medicinische Beobachtungen über Gnadenthal in Süd-Afrika.

8. Tübingen, 1856.

ROSLER (G. F.). Beyträge zur Naturgeschichte des Herzogthums Würtemberg.

$8^{\circ}$. Tü̈ingen, 1788-91.

ROSNY (L. de). Traité de l'Education des Vers à Soie au Japon.

8.. Paris, 1869.

ROSS (Sir J. C.). See 'Erebus' and 'Terror.'

ROSSE (J. C.). See 'CoRwin.' 
ROSSI (F.). Systematisches Verzeichniss der Tagfalter Schwärmer und Spinner des Erzherzogthums Oesterreich.

$8^{\circ}$. Wien, 1842.

ROSSI (F.). Systematisches Verzeichniss der zweiflügelischen Insecten.

8․ Wien, 1848.

ROSSI (P.). Fauna Etrusca.

2 vols. $4^{\circ} . \quad$ Liburni, 1790.

ROSSI (P.). Fauna Etrusca.

1. Mantissæ priore parte adjecta, iterum edita et aucta a J. C. L. HeLLwiG.

2. Iterum edita et aucta C. IlLIGER.

2 vols. $8^{\circ}$. Helmstadii, 1795-1807.

ROSSI (P.). Mantissa Insectorum, exhibens species nuper in Etruria collectas ; adjectis Faunæ Etruscæ illustrationibus ac emendationibus. $\quad 2$ vols. $4^{\circ}$. Pisis, 1792-94.

ROSSMÄSSLER (E. A.). Diagnoses Conchyliorum.

8. Dresden \& Leipzig, 1833.

ROSSMÄSSLER (E. A.). Iconographie der Land- und Süsswasser-Mollusken.

2 vols. $8^{\circ}$. Dresclen \& Leipzig, 1837-38.

ROSSMÄSSLER (E. A.). Iconographie der Europäischen Land- und Süsswasser-Mollusken. Fortgesetzt von Dr. W. KовеLT.

Neue Folge. Bd. I. Lief. 1-2. $\quad 8^{\circ}$. Wiesbaden, 1882.

ROTH (J. R.). Molluscorum Species.

4․ Monachii, 1839.

ROTH (J. R.). Spicilegium Molluscorum.

$8^{\circ}$. Cassellis, 1855 .

ROTH (J. R.). See Mousson (A.).

ROUSSEAU (L. F. E.). Anatomie Comparée du Système Dentaire chez l'Homme et chez les Principaux Animaux.

8.. Paris, 1827.

Nouvelle édition. $\quad 8^{\circ}$. Paris, 1839. 
ROUSSET DE MISSEY (J.). Observations sur l'origine, la constitution, et la nature des Vers-de-Mer, qui percent les Vaisseaux, les Pilliers, \&c. $8^{\circ}$. La Haye, 1733.

ROUX (P.). Ornithologie Provençale.

$4^{\circ}$. Marseille, 1825-29.

ROUX (P.). Crustacés de la Méditerranée, et de son littoral. $4^{\circ}$. Paris, $1828-30$.

ROUX (P.). Mémoire sur la Classification des Crustacés de la tribu des Salicoques. 8.. Marseille, 1831.

ROWLEY (G. D.). Ornithological Miscellany. 3 vols. $4^{\circ}$. London, 1875-78.

ROYAL SOCIETY. Memoirs for a Natural History of Animals. Published by an Order of Council of the Royal Society. $4^{\circ}$. London, 1701.

RUDOLPH (The Archduke, Crown Prince of Austria). Eine Orientreise. 8. Wien, 1881.

RUDOLPHI (C. A.). Entozoorum sive Vermium intestinalium Historia naturalis.

3 vols. $8^{\circ}$. Amsteloctami, 1808-10.

RUDOLPHI (C. A.). Beyträge zur Anthropologie.

8. Berlin, 1812.

RUDOLPHI (C. A.). Entozoorum Synopsis.

8. . Berolini, 1819.

RUMPHIUS (G. E.). Thesaurus Imaginum Piscium Testaceorum.

fol. Lugduni Batavorum, 1711.

RUMPHIUS (G. E.). Amboinische Raritäten-Kammer, oder Abhandlung von den.... Schnecken und Muscheln: aus dem Holländischen übersetzt von P.L. S. MüLLER ; mit Zusätzen von J. H. Chimsitz. fol. Wien, 1766.

RÜPPELL (E.). Atlas zu der Reise im nördlichen Afrika. Fische des Rothen Meers. [Uncoloured.]

The same, coloured. fol. Frankfurt am Main, 1828. 
RÜPPELL (E.). Abbildung und Beschreibung einiger neuen Versteinerungen von Solenhofen.

4\%. Frankfurt am Main, 1829.

RÜPPELL (E.). Beschreibung und Abbildung mehrerer neuer Fische im Nil entdeckt.

$4^{\circ}$. Frankfurt am Main, 1829.

RÜPPELL (E.). Fortsetzung der Beschreibung und Abbildung mehrerer neuer Fische. $4^{\circ}$. Frankfurt, 1832.

RÜPPELL (E.). Beschreibung und Abbildung von 24 Arten kurzschwänzigen Krabben.

$4^{\circ}$. Frankfurt am Main, 1830.

RÜPPELL (E.). Neuer Nachtrag von Beschreibungen und Abbildungen neuer Fische im Nil entdeckt.

$4^{\circ}$. Franlfurt am Main, 1836.

RŮPPELL (E.). Neue Wirbelthiere zu der Fauna von Abyssinien gehörig. fol. Frankfurt am Main, 1835-40.

RÜPPELL (E.). Ornithologische Miscellen (Cygnus, Ceblepyris, und Colius). $4^{\circ}$. Frankfurt am Main, 1839.

RÜPPELL (E.). Säugethiere aus der Ordnung der Nager. $4^{\circ}$. Frankfurt am Main, 1842.

RÜPPELL (E.). Beschreibung Abyssinischer Vögel aus der Ordnung der Klettervögel.

4. Frankfurt am Main, 1842.

RÜPPELL (E.). Systematische Uebersicht der Vögel Nord-ost-Africa's. roy. $8^{\circ}$. Frankfurt am Main, 1845.

RÜPPELL (E.). Beschreibung und Abbildung einer neuen Cinnyris. $\quad 4^{\circ}$. Frankfurt am Main, 1845.

RÜPPELL (E.). See Musedm Senckenbergiandm.

RUSCONI (G. B.). Descrizione Anatomica delle Salamandre Acquatiche. $\quad 4^{\circ}$. Pavia, 1817.

RUSCONI (M.). See Configliachi (P.). 
RUSSELL (A.). The Salmon.

8. Edinburgh, 1864.

RUSSELL (P.). Indian Serpents collected on the Coast of Coromandel. 2 vols. fol. London, 1796-1801.

RUSSELL (P.). Descriptions and Figures of Two hundred Fishes. fol. London, 1803.

RUSSELL (W. H.). See Tweeddale (Marquis of).

RUTHE (J. F.). See Wieguann (A. F. A.).

RÜTIMEYER (L.). Einige weitere Beiträge über das zahme Schwein und das Hausrind. $\quad 8^{\circ}$. Basel, 1878.

RÜTIMEYER (L.). Vom Meer bis nach den Alpen.

8. Bern, 1854.

RÜTIMEYER (L.). Dio Fauna der Pfahlbauten in der Schweiz. $4^{\circ}$. Basel, 1861.

RÜTIMEYER (L.). Crania Helvetica. Sammlung schweizerischer Schädelformen : bearbeitet von W. His.

40. Basel \& Genf, 1864.

RÜTIMEYER (L.). Die fossilen Schildkröten von Solothurn und der übrigen Juraformation. $4^{\circ}$. Zürich, 1873.

RÜTIMEYER (L.). Die Veränderungen der Thierwelt in der Schweiz seit Anwesenheit des Menschen.

8. Basel, 1875.

RÜTIMEYER (L.). Ueber die Art des Fortschritts in den organischen Geschöpfen. $\quad$ 8․ Basel \& Genf, 1876.

RÜTIMEYER (L.). Schädel von Esel und von Rind aus den Pfahlbauten von Auvernier und Sutz.

4\%. Basel, 1876.

RÜTIMEYER (L.). Ueber Pliocen- und Eisperiode auf beiden Seiten der Alpen.

4\%. Basel, 1876.

RÜTIMEYER (L.). Die Rinder der Tertiär-Epoche. Natürliche Geschichte der Antilopen. $\quad 4^{\circ}$. Zürich, 1877. 
RÜTIMEYER (L.). Beiträge zu einer Natürlichen Greschichte der Hirsche.

$4^{\circ}$. Zürich, 1881.

RUYSCH (H.). Theatrum universale omnium Animalium. 2 vols. fol. Amsterdami, 1718.

RYE (E. C.). British Beetles. $\quad$ 80. London, 1866.

RYE (E. C.). See Perrodicals: London, Zoological Record.

S.

SACHS (C.). Untersuchungen am Zitteraal Gymnotus electricus. Bearbeitet von E. DO Bols-REYMoND.

8․ Leipzig, 1881.

SADELIN (P. U.). Fauna Fennica. $\quad 8^{\circ} . \quad$ Aboce, 1810.

SAHLBERG (C. R.). Periculi Entomographici Species Insectorum nondum descriptas proposituri Fasciculus.

8. Abocie, 1823.

SAHLBERG (C. R.). Insecta Fennica.

8.. Helsingforsice, 1834.

SAIGEY (J. F.) \& SONNET (?). Eléments des Sciences physiques et naturelles.

12. . Paris, 1853.

SAINT-FARGEAU (A. Lepeletier de). Monographia Tenthredinetarum Synonimia extricata. $\quad 8^{\circ}$. Paris, 1823.

SAINT-FARGEAU (A. Lepeletier de). Histoire naturello des Insectes (Hyménoptères).

4 vols. $8^{\circ}$. Paris, 1836-46.

SAINT-FARGEAU (A. Lepeletier de). Seo FAUNe FranģaIse.

SAINT GEORGE (A. de la Valette). Symbolæ ad Trematodum Evolutionis historiam. $\quad 4^{\circ}$. Berolini, 1855. 
Saint-Hilaire (A. B.). See Levaillant (F.).

SAINT-HILAIRE (J. Barthélemy). See ARIstoteles.

ST. JOHN (S.). Life in the Forests of the Far East; or travels in Northern Borneo.

2nd edition. 2 vols. $8^{\circ}$. London, 1863.

ST. JOHN (C.). Natural History and Sport in Moray. roy. $8^{\circ}$. Edinburgh, 1882.

SAINT-SIMION (A. de). Miscellanées Malacologiques.

$8^{\circ}$. Toulouse, $1848-56$.

SALERNE (F.). Histoire Naturelle éclaircie dans une de ses parties principales, l'Ornithologie. $\quad 4^{\circ}$. Paris, 1767.

SALNOVE (R. de). La Vénerie Royale.

$4^{\circ}$. Paris, 1665.

SALVADORI (T.). Catalogo degli Uccelli di Sardegna.

8․ Milano, 1864.

SALVADORI (T.). Catalogo Sistematico degli Uccelli di Borneo, con note ed osservazioni di G. Doria ed O. Beccarr.

$8^{\circ}$. Genova, 1874.

SALVADORI (T.). Ornitologia della Papuasia e delle Molucche. 3 vols. $4^{\circ}$. Torino, 1880-81.

SALVADORI (T.). See 'Challenger.'

SALVADORI (T.). See FAUNA D' Italia.

SALVIANI (H.). Aquatilium animalium historia. fol. Romw, 1554.

SALVIN (F. H.) \& BRODRICK (W.). Falconry in the British Isles. 2nd edition. $8^{\circ}$. London, 1873.

SALVIN (0.). Index of Genera and Species referred to in 'The Ibis.' $\quad 8^{\circ}$. London, 1879. 
SALVIN (0.). A Catalogue of the Collection of Birds formed by the late H. E. Strickland.

$8^{\circ}$. Cambridge, 1882.

SalviN (0.). See Biologia Centrali-Americaxa.

SALVIN (0.). See 'ChalleNger.'

SAlviN (0.). See Pertodicals : London. The Ibis.

SALVIN (0.). See Sclater (P. L.).

'SAMARANG,' The Zoology of the Voyage of H.M.S., under the command of Capt. Sir E. Belcher, 1843-46. Edited by ARTHUR ADAurs.

Vertebrata. By J. E. GraY.

Fishes. By J. Richardson. $4^{\circ}$. London, 1850. 1849 .

Mollusca. By Arthur Adays \& Lovell Reeve. 1848. 1848.

Crustacea. By Arthor AdaMs \& Adam White.

1848.

SAMOUELLE (G.). The Entomologist's Useful Compendium.

8. London, 1819.

SAMOUELLE (G.). General Directions for collecting and preserving Exotic Insects and Crustacea.

$12^{\circ}$. Lonclon, 1826.

SAMOUELLE (G.). The Entomological Cabinet, being a natural history of British Insects. 120. London, 1833-34.

SAMUELS (E. A.). Descriptive Catalogue of the Birds of Massachusetts.

$8^{\circ}$. Boston, 1864.

SAMUELSON (J.) \& HICKS (J. B.). Humble Creatures. The Earthworm and the common House-fly.

8. Lonclon, 1858.

SANCTIS (L. de). Embryogenia degli organi elettrici delle Torpedini o degli organi pseudo-elettrici delle Raie. 4. Napoli, 1872.

SANCTIS (L. de). Monografia Zootomico-Zoologica sul Capidoglio. $4^{\circ}$. Roma, 1881. 
SAND (M.). Catalogue Raisonné des Lépidoptères du Berry et de l'Auvergne. $\quad 8^{\circ}$. Paris, 1879.

SANDER-RANG (A.). Histoire Naturelle des Aplysiens. Première famille de l'ordre des Tectibranches. Ouvrage servant de Complément à l'Hist. Nat. des Mollusques par Ferdssac.

fol. Puris, 1828.

SANDER-RANG (A.). Manuel des Mollusques.

16. Paris, 1829.

SANDERSON (G. P.). Thirteen Years among the Wild Beasts of India. 2nd edition. $4^{\circ}$. London, 1879.

SANDIFORT (E.). Museum anatomicum Academiæ Lugduno-Bataræ. 4 vols. fol. Lugduni Batavorum, 1793.

SANDIFORT (G.). Tabulæ craniorum diversarum nationum.

fol. Lugduni Batavorum, 1838-43.

SAPPEY (P. C.). Recherches sur l'Appareil Respiratoire des Oiseaux.

4. Paris, 1847.

SAPPEY (P. C.). L'appareil mucipare et le Système Lymphatique des Poissons. fol. Paris, 1880.

SARS (G. O.). Histoire Naturelle des Crustacés d'Eau douce de Norvège.

I. Les Malacostracés. $\quad 4^{\circ}$. Christiania, 1867.

SARS (G. O.). Indberetninger til Departementet for det Indre. [Fiskeri beretninger.] $8^{\circ}$. Christiania, 1869.

SARS (G. O.). On some remarkable forms of Animal Life. Parts 1 \& 2. $\quad 4^{\circ}$. Christiania, 1872-75.

SARS (G. O.). Syv nyo Cumaceer fra Vestindien.

$4^{\circ}$. Stockholm, 1873.

SARS (G. O.). Bidrag til Kundskaben om Norges Arktiske Fauna. $\quad 8{ }^{\circ}$. Christiania, 1878.

SARS (G. O.). Nye Bidrag til Kundskaben om Middelhavets Invertebratfauna Middelhavets Cumaceer.

$8^{\circ}$. Kristiania, 1879. 
SARS (M.). Beskrivelser og Jagttagelser over nogle mærkelige eller nye i Havet ved den Bergenske Ryst levende Dyr af Polypernes, Acalephernes, \&c. $\quad 4^{\circ}$. Bergen, 1835.

SARS (M.). Bidrag til Kundskaben om Middelhavets Littoral-Fauna.

8. Christiania, 1857.

SARS (M.). Oversigt af Norges Echinodermer.

8. Christiania, 1861.

SARS (M.). Mémoires pour servir à la connaissance des Crinoïdes vivants.

$4^{\circ}$. Christiania, 1868.

SARS (M.), KOREN (J. J.), \& DANIELSSEN (D. C.). Fauna littoralis Norvegiæ. fol. Christiania, 1846.

SARS (M. \& G. O.). Bidrag til Kundskabom Christianiafjordens Fauna.

8. Christiania, 1868-70.

SASSI (A.). Saggio sopra i Pesci, Rettili, e Mammiferi della Liguria.

$8^{\circ}$. Genova, 1846.

SATCHELL (T.). See Westwood (T.).

SAULCY (F. de). See Bodrguignat (J. R.).

SAUNDERS (E.). Insecta Saundersiana. Buprestidce, Part 1. 8 . London, 1869.

SAUNDERS (E.). Species of the Genus Buprestis of Linneus described previous to $1830 . \quad 8^{\circ}$. London, 1870.

SAUNDERS (E.). Catalogus Buprestidarum. 8. . London, 1871.

SAUNDERS (H.). See 'Challenger.'

SAUNDERS (H.). See Periodicals: London. The Ibis.

SAUNDERS (H.). See YARRELL。

SAUNDERS (W. W.). Insecta Saundersiana. Diptera by F. WALKer. Coleoptera by H. Jekel.

8'. London, 1850-55. 
SAUNDERS (W.) \& REED (E. B.). Report on the Colorado Potato-Beetle. 8. Toronto, 1871.

SAUNDERS (W.). Insects Injurious to Fruits.

8. Philadelphia, 1883.

SAUSSURE (H. de). Monographie des Guêpes Solitaires. Euméniens. 8․ Genève \& Paris, 1852.

SAUSSURE (H. de). Études sur la Famille des Vespides. Monographie des Guêpes Sociales.

8. Paris \& Genève, 1853.

SAUSSURE (H. de). Études sur la Famille des Vespides. Monographio des Masariens et supplément aux Euméniens.

8. Paris \& Genève, 1854.

SAUSSURE (H. de). See 'Novara.'

SAUSSURE (H. de). See Surveys \& Explorations : France.

SAUSSURE (H. de) \& SICHEL (J.). Catalogus Specierum Generis Scolia. $8^{\circ}$. Geneva \& Parisiis, 1864.

SAUVAGE (H. E.). Bibliothèque Instructive. La Grande Pêche (Les Poissons).

8.. Paris, 1883.

SAVI (P.). Catalogo degli Uccelli della Provincia Pisana. $8^{\circ}$. Pisa, 1823.

SAVI (P.). Ornitologia Toscana.

80. Pisa, 1827.

SAVI (P.). Ornitologia Italiana.

3 vols. $8^{\circ}$. Firenze, 1873-77.

SAVIGNY (J. C.). Histoire Naturelle et Mythologique de l'Ibis.

8. Paris, 1805.

SAVIGNY (J. C.). Mémoires sur les Animaux sans Vertèbres.

2 vols. in 1. $8^{\circ}$. Paris, 1816.

SAVIGNY (J.C.). See Periodicals : London. Willughby Society. 
SAVIGNY (J. C.) \& AUDOUIN (V.). Crustacea of Egypt. A collection of figures only. Plates I.-XII. 4\%.

SAXBY (H. L.). The Birds of Shetland. Edited by S. H. SAXBY.

8. Edinburgh, 1874.

SAY (T.). Description of the Land and Freshwater Shells of the United States. $\quad 8^{\circ}$. Philadelphia, 1819.

SAY (T.). American Entomology. 3 vols. $8^{\circ}$. Philadelphia, 1824.

SAY (T.). Descriptions of Terrestrial Shells of N. America. 8. Philadelphia, 1856.

SAY (T.). The Complete Writings of Thomas Say on the Conchology of the United States. Edited by W. G. BrrNEY. 8. New York, 1858.

SAY (T.). American Entomology. Edited by J. L. LE Conte. 2 vols. $8^{\circ}$. New York, 1869.

SCACCHI (A.). Catalogus Conchyliorum regni Neapolitani. 8. Neapoli, 1836.

SCAMMON (C. M.). The Marine Mammals of the Northwestern Coast of North America.

40. San Francisco, 1874.

SCATTAGLIA (P.) \& ALESSANDRI(J.). Descrizione degli Animali Quadrupedi. 4 vols. fol. Venezia, 1771-75.

SCHACHT (H.). Die Vogelwelt des T'eutoburger Waldes. 8. Detmolil, 1877.

SCHÄFER (M.). Moselfauna. Erster Theil. Wirbelthiere.

8. Trier, 1844.

SCHÄFFER (J. C.). Die Armpolypen in den süssen Wassern um Regensburg. $\quad 4^{\circ}$. Regensburg, 1754. 2nd edition. $4^{\circ}$. Regensbury, 1763. 
SCH $\ddot{A F F E R ~(J . ~ C .) . ~ P i s c i u m ~ B a v a r i c o-R a t i s b o n e n s i u m ~}$ pentas. $4^{\circ}$. Ratisỏonce, 1761.

SCHÄFFER (J. C.). Zweifel und Schwürigkeiten welche in der Insectenlehre an' noch vorwalten.

4․ Regensburg, 1766.

SCH ̈̈FFER (J. C.). Fernere Zweifel und Schwierigkeiten welche in der Insectenlehre an' noch rorwalten.

$4^{\circ}$. Regensburg, 1766.

SCHÄFFER (J. C.). Icones Insectorum circa Ratisbonam indigenorum.

5 rols. $4^{\circ}$. Regensourg, 1766.

SCḦ̈̈FER (J. C.). Elementa Entomologica, $4^{\circ}$. Regensburg, 1766.

SCH ÄFFER (J. C.). Elementa Ornithologica Iconibus illustrata.

$4^{\circ}$. Ratisbona, 1774 .

2nd edition. $4^{\circ} . \quad$ Ratisbonce, 1779.

SCHÄFFER (J. C.). Abbildung und Beschreibung des Mayenwurmkäfers, als eines zuverlässigen Hülfsmittels wider den tollen Hundebiss.

$4^{\circ}$. Regensburg, 1778.

SCHÄFFER (J. C.). Icones Insectorum Ratisbonensium illustratæ et indice auctæ a G. W. Panzero.

Editio nova. 4 vols. $4^{\circ}$. Erlangae, 1804.

SCHAUFUSS (L. W.). Nunquam otiosus.

8. Dresden, 1870-79.

SCHAOM (H.). Verzeichniss der Lamellicornia Melitophila. 16. Stettin, 1848.

SCHAOM (H.). Catalogus Coleopterorum Europæ.

8०. Berlin, 1859.

SCHACM (H. R.). Symbolæ ad Monographiam Scydmænorum, Insectorum generis.

8. Halis, 1841.

SCHELHAMER (G. C.). Anatomes Xiphiae Piscis.... brevis enarratio.

$4^{\circ}$. Hamburgi, 1707. 
SCHELLENBERG (J. R.). Helvetische Entomologie.

2 vols. $8^{\circ}$. Züirich, 1798-1806.

SCHELLENBERG (J. R.). Cimicum in Helvetiæ aquis et terris degentium genus.

$8^{\circ}$. Turici, 1800.

SCHELLENBERG (J. R.). Entomologische Beyträge. Heft I. $4^{\circ}$. Winterthur, 1802.

SCHELLENBERG (J. R.). Genres des Nouches Diptères. Gattungen der Fliegen. 8․ Zürich, 1803.

SCHEMBRI (A.). Catalogo Ornitologico del gruppo di Malta. $8^{\circ}$. Malta, 1843.

SCHEMBRI (A.). Vocabolario dei Sinonimi dell' Ornitologia Europea.

8. Bologna, 1846.

SCHENK (S. Lo). Lehrbuch der vergleichenden Embryologie der Wirbelthiere.

$8^{\circ}$. Wien, 1874.

SCHERZER (K.). Narrative of the Circumnarigation of the Globe by the Austrian Frigate ' Novara.'

See 'Novara.'

3 vols. $\quad 8^{\circ}$. London, 1861-63.

SCHIESS (H.). Versuch einer speziellen Neurologie der Rana esculenta. $4^{\circ}$. St. Gallen \& Bern, 1857.

SCHIFFERMÜLLER (J.) \& DENIS (M.). Systematische Kenntniss der Schmetterlinge der Wienergegend, herausgegeben von einigen Lehrern am K. $\mathbb{K}$. Theresianum.

$4^{\circ}$. Wien, 1776 .

SCHILDE (J.). Gegen pseudodoxische Transmutationslehren, ein Entomolog.

8.. Leipzig, 1879.

SCHINER (J. R.). Fauna Austriaca. Die Fliegen (Diptera).

2 vols. $8^{\circ}$. Wien, 1862-64.

SCHINER (J. R.). See 'Novara.'

SCHINZ (H. R.). Verzeichniss der in der Schweiz vorkommenden Wirbelthiere. (Erster Theil der Fauna Helvetica.)

$4^{\circ}$. Neuchatel, 1837. 
SCHINZ (H. R.). Europäische Fauna, oder Verzeichniss der Wirbelthiere Europa's. 2 vols. $8^{\circ}$. Stuttgart, 1840.

SCHINZ (H. R.). Systematisches Verzeichniss aller bis jetzt bekannten Säugethiere, oder Synopsis Mammalium.

2 vols. $8^{\circ}$. Solothurn, 1844-45.

SCHINZ (H. R.). See MEISNER (F.).

SCHIÖDTE (J. C.). Genera og Species af Danmarks Eleutherata. 8. Kjöbenhavn, 1841.

.SCHIÖDTE (J. C.). Corotoca og Spirachtha : Staphyliner. $4^{\circ}$. Kjöbenhavn, 1854.

SCHIÖDTE (J. C.). See Periodicals : Copenhagen.

SCHIÖDTE (J. C.). Danmarks Cerambyces.

8. Kjöbenhavn, 1864.

SCHIÖDTE (J. C.). Zoologia Danica.

Hefte I.-III. fol. Kjobenhavn, 1878-82.

SCHLAEFLI (A.). See Moosson (A.).

SCHLECHTENDAL (D. H. R.) \& WÜNSCHE (O.). Die Insecten. Abtheil. I.-III. 80. Leipzig, 1879.

SCHLEGEL (H.). Atlas van Platen behoorende bij den Leercursus der Natuurlijke Historie. fol. Leyden, n. d.

SCHLEGEL (H.). Verhandeling over eenen Vinvisch. $4^{\circ}$. Leiden, 1831.

SCHLEGEL (H.). Essai sur la physionomie des Serpens. 3 vols. Text, $8^{\circ}$. Atlas, $4^{\circ}$. La Haye, 1837.

SCHLEGEL (H.). Essay on the physiognomy of Serpents, translated by T. S. Traill. $\quad 8^{\circ}$. Edinburgh, 1843.

SCHLEGEL (H.). Abhandlungen aus dem Gebiete der Zoologie und vergleichenden Anatomie. $4^{\circ}$. Leiden, 1841. 
SCHLEGEL (H.). De Diergaarde en het Museum van het Genootschap "Natura Artis Magistra," te Amsterdam.

8. Amsterdam, 1842.

SCHLEGEL (H.). Revue critique des Oiseaux d'Europe. Kritische Übersicht der Europäischen Vögel.

8․ Leiden, 1844.

SCHLEGEL (H.). Verhandeling over de Vereischten van Natuurkundige Afbeeldingen.

4\%. Haarlem, 1849.

SCHLEGEL (H.). Over den groei en de kleurveranderingen der Vederen van de Vogels. $\quad 8^{\circ}$. Leiden, 1853.

SCHLEGEL (H.). Handleiding tot de beoefening der Dierkunde.

2 vols. $8^{\circ}$. Breda, 1857.

SCHLEGEL (H.). Le genre Corvus.

$4^{\circ}$. Amsterdam, 1859.

SCHLEGEL (H.). La pie aux ailes brunes, Pica pyrrhoptera.

$4^{\circ}$. Amsterdam, 1859.

SCHLEGEL (H.). De Toerako's.

fol. Amsterdam, 1860.

SCHLEGEL (H.). De Vogels van Nederlandsch Indië.

$4^{\circ}$. Haarlem, 1863.

SCHLEGEL (H.). Monographie des Singes.

8.. Liide, 1876.

SCHLEGEL (H.). De Vogels van Nederland.

2 vols. $8^{\circ}$. Amsterdam, 1878.

SCHLEGEL (H.) \& POLLEN (F. P. L.). Recherches sur la Faune de Madagascar. Mammifères et Oiseaux.

$4^{\circ}$. Leyde, 1868.

SCHLEGEL (H.) \& WITKAMP (P. H.). De Dierentuin van het koninklijk zoologisch Genootschap "Natura Artis Magistra " te Amsterdam. $4^{\circ}$. Amsterdam, 1872.

SCHLEGEL (H.) \& WULVERHORST (A.H. V.). Traité de Fauconnerie. fol. Leiden, 1844-53. 
SCHLEGEL (H.). See Periodicals: Amsterdam.

SCHLEGEL (H.). See Periodicals : Leiden.

SCHLEGEL (H.)。 See Srebold (C. T. von).

SCHLEGEL (H.). See Temmincr (C. J.).

SCHLOESSER (W.). See Espinas (A.).

SCHLUESSER (G.). De Petromyzontum et Anguillarum sexu. 8. Dorpati Livonorum, 1848.

SCHLUGA (J. B.). Primae Lineæ cognitionis Insectorum. 8. . Bibliopolce Viennensis, 1767.

SCHLÜTER (F.). Kurzgefasstes systematisches Verzeichniss meiner Conchyliensammlung. $\quad 8^{\circ}$. Halle, 1838.

SCHMALTZ(C. S. R.). See Rafinesque Schmaltz (C. S.)。

SCHMALZ (E.). De Entozoorum Systemate Nervoso. 8.. Lipsice, 1827.

SCHMALZ (E.). XIX. Tabulæ Anatomiam Entozoorum illustrantes congestæ, nec non explicatione præditæ.

4. Dresdce \& Lipsice, 1831.

SCHMARDA (L. K.). Kleine Beiträge zur Naturgeschichte der Infusorien. $4^{\circ}$. Wien, 1846.

SCHMARDA (L. K.). Die geographische Verbreitung der Thiere. 8. Wien, 1853.

SCHMARIAA (L. K.). Neue wirbellose Thiere: Turbellarien, Rotatorien und Anneliden. $4^{\circ}$. Leipzig, 1859-61.

SCHMARDA (L. K.). Die Maritime Production der österreichischen Küstenländer. $\quad 8^{\circ}$. Wien, 1864-67.

SCHMARDA (L. K.). Die Cultur des Meeres in Frankreich. 8. Wien, 1869. 
SCHMARDA (L. K.). Zoologie.

2 vols. $8^{\circ}$. Wien, 1877-78.

SCHMELTZ (J. D. E.). Verzeichniss verkäuflicher Holothuriden. $8^{\circ}$. Hamburg, 1870 .

SCHMIDBERGER (J.). Beiträge zur Obstbaumzucht und zur Naturgeschichte der den Obstbäumen schädlichen Insekten.

4 vols. $8^{\circ}$. Linz, 1827-36.

SCHMIDT (E. O.). Die rhabdocoelen Strudelwürmer (Turbellaria rhabdocoela) des süssen Wassers.

$8^{\circ}$. Jena, 1848.

SCHMIDT (E. O.). Neue Beiträge zur Naturgeschichte der Würmer.

$8^{\circ}$. Jena, 1848.

SCHMID (K.). Naturhistorische Beschreibung der Säugethiere. $4^{\circ}$. München, 1818.

SCHMID (K.). Naturhistorische Beschreibung der Vögel. $4^{\circ}$. München, 1818.

SCHMIDT (M. F.). Reisen im Amur-Lande und auf der Insel Sachalin. $4^{\circ}$. St. Petersburg, 1868.

SCHMIDT (M. F.). Wissenschaftliche Resultate der zur Aufsuchung eines angekündigten Mammuthcadavers an den untern Jenissei ausgesandten Expedition.

4\%. St. Petersburg, 1872.

SCHMIDT (M. F.). Ueber die Petrefakten der Kreideformation von Sachalin.

$4^{\circ}$. St. Petersburg, 1873.

SCHMIDT (M. F.). Ueber die Russischen silurischen Leperditien. $4^{\circ}$. St. Petersburg, 1873.

SCHMIDT (M. F.). Ueber einige neue Baltischsilurische Petrefacten. $\quad 4^{\circ}$. St. Petersburg, 1874.

SCHMIDT (O.). Diagnosen neuer Fröscho des Zoologischen Cabinets zu Krakau.

$8^{\circ}$. Wien, 1857.

SCHMIDT (O.). Deliciae Herpetologicae musei Zoologici Cracoviensis. Ungeschwänzten Batrachier.

$4^{\circ}$. Wien, 1858. 
SCHMIDT (O.). Die Spongien des Adriatischen Meeres. 4. Leipzig, 1862-66.

SCHMIDT (0.). Die Spongien der Küste von Algier.

$4^{\circ}$. Leipzig, 1868.

SCHMiDT (O.). Grundzüge einer Spongien-Fauna des Atlantischen Gebietes.

4. Leipzig, 1870.

SCHMIDT (O.). Die Spongien des Meerbusen ron Mexico und des Carabischen Meeres.

$4^{\circ}$. Jena, 1880.

SCHMIDT-GOEBEL (H. M.). De Pselaphis Faunæ Pragensis, cum anatomia Clavigeri. $\quad 8^{\circ}$. Praga, 1836.

SCHMIDT-GOEBEL (H. M.). Beytrag zu einer Monographie der Pselaphen, enthaItend nene Species aus Asien. 8. Prag, 1838.

SCHMIDT-GOEBEL (H. M.). Fauna Coleopterorum Birmaniæ.

8. Prague, 1846.

SCHMIEDEKNECHT (H. L. O.). Apidæ Europeæ.

Fasc. I.-IV. 80. Gumperda, 1882. Fasc. V.-VII. 8 . Berolini, 1883.

SCHMIIEDLEIN (G. B.). Specimen Faunæ Insectorum Lipsicæ. $12^{\circ}$. Lipsice, 1790.

SCHNEIDER (A.). Monographie der Nematoden.

$8^{\circ}$. Berlin, 1866.

SCHNEIDER (A.). Beiträge zur vergleichenden Anatomie und Entwickelungsgeschichte der Wirbelthiere.

$4^{\circ}$. Berlin, 1879 .

SCHNEIDER (A.). Das Ei und seine Befruchtung.

$4^{\circ}$. Breslau, 1883.

SCHNEIDER (A.). Zoologische Beiträge.

Bd. I. Hft. 1. $8^{\circ}$. Breslau, 1883 .

SCHNEIDER (D. H.). Neuestes Magazin für die Liebhaber der Entomologie. $\quad 12^{\circ}$. Stralsund, 1791-94. 
SCHNEIDER (D. H.). Verzeichniss der zur Verlassenschaft des Herrn Senator Schneider gehörigen Insectensammlung.

$12^{\circ}$. Stralsund, 1828.

SCHNEIDER (G. T.). Monographia generis Rhaphidice Linnæi. $4^{\circ}$. Vratislavice, 1843.

SCHNEIDER (G. T.). Symbolæ ad monographiam generis Chrysopce, Leach.

$8^{\circ}$. Vratislavice, 1851.

SCHNEIDER (H.). Ueber die Augenmuskelnerren der Ganoiden.

$8^{\circ}$. Jena, 1881.

SCHNEIDER (J. G.). Ichthyologiæ Veterum Specimina. $4^{\circ}$. Francofurti, 1782 .

SCHNEIDER (J. G.). Allgemeino Naturgeschichte der Schildkröten. 8. Eeipzig, 1783.

SCHNEIDER (J. G.). Sammlung vermischter Abhandlungen zur Ausklärung der Zoologie. $\quad 8^{\circ}$. Berlin, 1784.

SCHNEIDER (J. G.). Amphibiorum Physiologiæ specimen primum.

Specimen alterum. Editio repetita.

$4^{\circ}$. Viadrum, 1790.

4. Zullichovia, 1797.

SCHNEIDER (J. G.). Beyträge zur Naturgeschichte der Wallfischarten.

8. Leipzig, 1795.

SCHNEIDER (J. G.). Sammlung von Anatomischen Aufsätzen und Bemerkungen zur Ausklärung der Fischkunde. Erster Theil. 8. Leipzig, 1795.

SCHNEIDER (J. G.). Historiæ Amphibiorum naturalis et literariæ, fasciculus primus. $\quad 8^{\circ}$. Jence, 1799-1801.

SCHNEIDER (J. G.). See Aliands.

SCHNEIDER (J. G.). See ArIstoteles.

SCHNEIDER (J. G.). See BLoch (M. E.).

SCHNEIDER (J. G.). See Monro (A.). 
SCHNEIDER (0.). Naturwissenschaftliche Beiträge zur Kenntniss der Kaukasusländer auf Grund seiner Sammelbeute. 8. Dresden, 1878.

SCHNITZLER (H. J.). De Oniscineis Agri Bonnensis.

8․ Colonice Agrippince, 1853.

SCHOEPFF (J. D.). Historia Testudinum iconibus illustrata. $4^{\circ}$. Eilangce, 1792.

SCHONEVELDE (S.). Ichthyologia et Nomenclaturæ animalium marinorum, fluviatilium, lacustrium, quæ in Ducatibus Slesvici et Holsatiæ, et Hamburgi occurrunt triviales. $4^{\circ}$. Hamburgi, 1624.

SCHÖNHERR (C. J.). Synonymia Insectorum.

2 vols. $8^{\circ}$. Stockholm, 1806.

SCHÖNHERR (C. J.). Curculionidum dispositio methodica. 8. Lipsice, 1826.

SCHÖNHERR (C. J.). Genera et species Curculionidum. 16 vols. $8^{\circ}$. Paris, 1833.

SCHÖNHERR (C. J.). Genera et species Curculionidum. Catalogus ab H. JeKeL recensus. $\quad 1^{\circ}$. Paris, 1849.

SCHRANK (F. von P.). Enumeratio Insectorum Austriæ indigenorum. 8. Augustce Vindelicorum, 1781.

SCHRANK (F. von P.). Fauna Boica. 3 vols. $8^{\circ}$. Nürnberg, 1798-1803.

SCHRANK (F. von P.). See SpIX (J. B.).

SCHREBER (J. C. D. von). Die Säugethiere. Theil 1-7. $4^{\circ}$. Leipzig, 1774-1834. Supplementbände 1-5. $4^{\circ}$. 1840-1855.

SCHREGER (C. H. T.). Versuch einer vergleichenden Anatomie des Auges und der Thränenorgane des Menschen. 8. Leipzig, 1810.

SCHREIBER (E.). Herpetologia Europæa.

8. Braunschweig, 1875. 
SCHREIBERS (K. v.). Nachrichten von den Kaiserl. Österreichischen Naturforschern in Brasilien.

8.. Brünn, 1820.

SCHREKENSTEIN (F. R. Von).

1. Verzeichniss der Schmetterlinge.

2. Verzeichniss der Käfer.

3. Verzeichniss der Halbkäfer.

3 parts. $8^{\circ}$. Tï̈bingen, 1800-1802.

SCHRENCK (L. จ.). Reisen und Forschungen im AmurLande in den Jahren 185t-1856.

$4^{\circ}$. St. Petersburg, 1858-76.

SCHRÖCKIO (L.). Historia Moschi ad normam Academiæ Naturæ Curiosorum.

$4^{\circ}$. Augustce Vindelicorum, 1782.

SCHROEDER VAN DER KOLK (J.L. C.). Mémoire sur l'Anatomie et la Physiologie du Gastrus equi.

4\%. Amsterdam, 1845.

SCHRÖTER (J. S.). Musei Gottwaldiani Testaceorum, Stellarum marinarum et Coralliorum quæ supersunt tabulæ. Die Conchylien, Seesterne und Meergewächse der Gottwaldtischen Naturaliensammlung, \&c. $\quad 4^{\circ}$. Nürnberg, 1782.

SCHULTZ (A.). Notice sur les Pêcheries et la Chasse aux Phoques dans la Mer Blanche, l'Océan Glaciale et la Mer Caspienne. $4^{\circ}$. St. Petersburg, 1873.

SCHULTZE (C. A. S.). Mikroskopische Untersuchungen über des Herrn Robert Brown's Entdeckung lebender, selbst im Feuer unzerstörbarer Theilchen in allen Körpern ; und über Erzeugung der Monaden.

$4^{\circ}$. Carlsruhe, 1828.

SCHULTZE (C. A. S.). Prodromus descriptionis formarum partium elementarium in animalibus. $4^{\circ}$. Berolini, 1828.

SCHULTZE (C. A. S.). Macrobiotus hufelandii, animal e crustaceorum classe novum.

$4^{\circ}$. Berolini, 1834.

SCHULTZE (F. E.). Ueber Cordylophora lacustris.

$4^{\circ}$. Leipzig, 1871. 
SCHULTZE (F. E.). Ueber Syncoryne sarsii und Sarsia tubulosa.

$4^{\circ}$. Leipzig, 1873.

SCHUL'TZE (M. J. S.). De Arteriarum notione.

8․ Gryphice, 1849.

SCHULTZE (M. S.). Observationes nonnullæ de Ovorum Ranarum segmentatione.

$4^{\circ}$. Bonnce, n. d.

SCHULTZE (M. S.). Beiträge zur Naturgeschichte der Turbellarien. $4^{\circ}$. Greifswald, 1851.

SCHULTZE (M. S.). Ueber den Organismus der Polythalamien (Foraminiferen). fol. Leipzig, 1854.

SCHULTZE (II. S.). Land-Planarien. $4^{\circ} . \quad$ Halle, 1857.

SCHULTZE (M. S.). Die Hyalonemen, ein Beitrag zur Naturgeschichte der Spongien. $4^{\circ}$. Bonn, 1860.

sCHUltZE (M. S.). See Periodicals : Bonn.

SCHULZ (J. H.). Fauna Marchica. $\quad 8^{\circ}$. Berlin, 1845.

SCHUMACHER (C. F.). Essai d'un Nouveau Système des Habitations des Vers Testacés. $\quad 4^{\circ}$. Copenhague, 1817.

SCHUMACHER (C. F.). Descriptio Musei Anthropologici Universitatis Hafniensis. $\quad 4^{\circ}$. Hafnice, 1828.

SCHUMANN (A.). Die Affenmenschen Carl Vogts.

8. Leipzig, 1868.

SCHWARZ (C.). Neuer Raupenkalender, oder Beschreibung aller bis jezt bekannten Europäischen Raupen.

2 vols. $8^{\circ}$. Nürnberg, 1791.

SCHWEDER ( ). Die Vögel der Ostseeprovinzen nach ihren Merkmalen.

$8^{\circ}$. Riga, 1881.

SCHWEIGGER (A. F.). Prodromi monographiæ Cheloniorum sectio prima et sectio secunda. $8^{\circ}$. Regiomonti, 1814. 
SCHWEIGGER (A. F.). Beobachtungen auf naturhistorischen Reisen.

4. Berlin, 1819.

SCHWEIGGER (A. F.). Handbuch der Ungegliederten Thiere.

8. Leipzig, 1820.

SCHWENCKFELD (C.). Theriotropheum Silesiæ.

8. Lignicii, 1603.

SCLATER (P. L.). Tanagrarum Catalogus Specificus.

8. Basingstoke, 1854.

SCLATER (P. L.). A Monograph of the Tanagrine genus Calliste.

8. London, 1857.

SCLATER (P. L.). Zoological Sketches by JoSEPH WoLF. 1st and 2nd series. 2 vols. fol. London, 1861.

SCLATER (P. L.). Catalogue of a collection of American Birds.

8. London, 1862.

SCLATER (P. L.). A Monograph of the Jacamars and Puff-Birds. (Galbulidae et Bucconidce.)

$4^{\circ}$. London, 1879-82.

SCLATER (P. L.). Nitzsch's Pterylography. See RAY SocIETY.

SClater (P. L.). See 'Challenger.'

SClateR (P. L.). See Periodicals : Londoin. The Ibis. SCLATER (P. L.) \& SALVIN (O.). Exotic Ornithology. $4^{\circ}$. London, 1869.

SCLATER (P. L.) \& SALVIN (O.). Nomenclator Avium Neotropicalium.

$4^{\circ}$. London, 1873.

SCOPOLI (G. A.). Entomologia Carniolica.

$8^{\circ}$. Vindobone, 1763. Forty-three additional plates; unpublished.

SCOPOLI (G. A.). See Periodicals: London. Willughby Society. 
SCOTT (A. W.). Australian Lepidoptera.

Parts I.-III. fol. London, 1864.

SCOTT (A. W.). Mammalia, recent and extinct.

8.. Sydney, 1873.

SCOTT (R. H.). See Fisheries : London. International Fisheries Exhibition, 1883.

SCOTT (W. B.). Beiträge zur Entwicklungsgeschichte der Petromyzonten. 8.. Leipzig, 1881.

SCOUTETTEN (H.). Études sur les Trichines.

8. Paris, 1866.

SCRIBA (L. G.). Beiträge zu der Insekten-Geschichte.

$4^{\circ}$. Frankfurt, 1790.

SCRIBA (L. G.). Journal für die Liebhaber der Entomologie. 8. Frankfurt, 1791.

SCROPE (W.). Days of Deer-Stalking in the Forest of Atholl. 3rd edition. $8^{\circ}$. London, 1847.

SCUDDER (S. H.). Catalogue of the Orthoptera of North America.

$8^{\circ}$. Washington, 1868.

SCUDDER (S. H.). Catalogue of Scientific Serials, 16331876.

8. Cambridge, U. S., 1879.

SCUDDER (S. H.). See Bibliographies.

SCUDDER (S. H.). See Pertodicals : Boston, U. S.

SEBA (A.). Locupletissimi rerum Naturalium Thesauri .... descriptio. 4 vols. fol. Amsterdami, 1734-65.

SEEBOHM (H.). Siberia in Europe. $8^{\circ}$. London, 1880.

SEEBOHM (H.). Siberia in Asia. $\quad 8^{\circ}$. London, 1882.

SEEBOHM (H.). A History of British Birds, with coloured illustrations of their Eggs.

Parts I. \& II. $8^{\circ}$. London, 1883. [In progress.] 
SEGUENZA (G.). Brevissimi cenni intorno le formazioni terziarie della provincia di Reggio-Calabria.

8.. Messina, 1877.

SEGUENZA (G.). Nuculidi Terziarie rinvenute nello provincie meridionali d' Italia.

$4^{\circ}$. Roma, 1877.

SEIDLITZ (G.). Die Bildungsgesetze der Vogeleier in histologischer und genetischer Beziehung und das Transmutationsgesetz der Organismen. $\quad 8^{\circ}$. Leipzig, 1869.

SEIDLITZ (G.). Die Darwin'sche Theorie.

8. Leipzig, 1875.

SEIDLITZ (G.). Beiträge zur Descendenz-Theorie.

8. Leipzig, 1876.

SELBY (P. J.). Illustrations of British Ornithology.

Text 2 vols. $8^{\circ}$, and plates 2 vols. fol. Edinburgh, 1833.

SELBY (P. J.). See Periodicals: London.

SELENKA (E.). Beiträge zur Anatomie und Systematik der Holothurien.

8.. Leipzig, 1867.

SELENKA (E.). Zoologische Studien.

I. Befruchtung des Eies von Toxopneustes variegatus.

II. Zur Entwickelungsgeschichte der Seeplanarien.

$4^{\circ}$. Leipzig, 1878-81.

SELENKA (E.). Studien über Entwickelungsgeschichto der Thiere.

Heft I. $4^{\circ}$. Wiesbaden, 1883.

SELENKA (E.). See Periodicals : Leiden.

SELENKA (E.). Sipunculiden.

See Surveys \& Explorations: Germany. Leipzig.

SELIGMANN (J. M.). Sammlung verschiedener ausländischer und seltener Vögel.

(See Catesby \& Edwards.)

9 vols. in 4. fol. Nurnberg, 1749.

SELLIUS (G.). Historia Naturalis Teredinis seu Xylophagi Marini, .... Belgici. $\quad 4^{\circ}$. Rhenum, 1733. 
SELOUS (F. C.). A Hunter's Wanderings in Africa.

8.. London, 1881.

SELYS-LONGCHAMPS (E. de). Essai Monographique

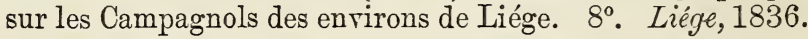

SELYS-LONGCHAMPS (E. de). Etudes de Micromammalogie: Musaraignes, Rats, et Campagnols. [With MS. Notes by Professor Reinhardt.] 80. Paris, 1839.

SELYS-LONGCHAMPS (E. de). Monographie des Libellulidées d'Europe. 8.. Paris, 1840.

SELYS-LONGCHAMPS (E. de). Faune Belge.

8․ Liége, 1842.

SELYS-LONGCHAMPS (E. de). Synopsis des Caloptérygines.

8०. Bruxelles, 1853.

SELYS-LONGCHAMCPS (E. de). Monographie des Gomphines.

8․ Bruxelles \& Leipzig, 1857.

SELYS-LONGCHAMPS (E. de). Synopsis des Cordulines. 8.. Bruxelles, 1871.

SELYS-LONGCHAMPS (E. de) \& HAGEN (H. A.). Rerue des Odonates ou Libellules d'Europe.

$8^{\circ}$. Bruxelles, Leipzig, \& Paris, 1850.

SEMPPER (C.). Die Philippinen und ihre Bewohner. Sechs Skizzen. 8. Würzburg, 1869.

SEMPER (C.). Der Haeckelismus in der Zoologie.

8. Hamburg, 1876.

SEMPER (C.). See Ton der Decken's Reisen.

SEMPER (C.). See Periodicals: Würzburg.

SEMPER (C.). Fieisen im Archipel der Philippinen.

See Surters \& Explorations: Germiny.

SENDEL (N.). Historia Succinorum corpora aliena involventium, \&c. fol. Lipsiae, 1742.

SENIOR (W.). See Fisheries: London. International Fisheries Exhibition, 1883. 
SEOANE (M.). See DictiorarIEs: Spanish-English.

SEPP (J. C.). Beschouwing der Wonderen Gods, in de minstgeachte schepzelen of Tederlandsche Insecten, naar hunne, \&c.

8 rols. $4^{\circ}$. Amsterdam, 1762.

SEPP (J. C.). Surinaamsche Tlinders. Papillons de Surinam. Text and plates. $4^{\circ}$. Amsterdam.

SERRES (M. de). Des Causes des Migrations des Animaux, et particulièrement des Oiseaux et des Poissons.

$4^{\circ}$. Haailem, 1842 .

SERTAIN (G.). Annales de Malacologie.

3 rols. so. Paris, 1 s 50 .

SERTILLE (А.). See Аугот (C. J. R.).

SERTILLE (A.). See Fatxe Fraxçaise.

SERTILLE (J. G. Audinet). See Atoinet-Sertilte.

SESLER (L.). See Dorati (T.).

SETSCHENOW (I.). Die Kohlensäure des Blutes.

40. St. Petersburg, 1Si9.

SEUBERT (M.). Symbolæ ad Erinacei europæi anatomen. $4^{\circ}$. Bonnce, 1841 .

SETERIN (J.). Tentamen Zoologiæ Hungaricæ.

12․ Posonii, 1779.

SEVERINCS (M. A.). De Viperæ Natura, Teneno, Medicina demonstrationes et experimenta nora.

$4^{\circ}$. Patavii, 1650.

SFORZINO DA CARCANO (F.). I tre libri degli Cocelli da Rapina. Con un trattato de' Cani da Caccia.

$12^{\circ}$. Vicenza, 1622.

SHARP (Capt.). See Dayrpier (W.).

SHARP (D.). The object and method of Zoological Nomenclature. Sondon, 1873. 
SHARPE (R. B.). A Monograph of the Alcedinida. $4^{\circ}$. London, 1868-71.

SHARPE (R. B.). Catalogue of African Birds in the collection of R. B. S.

8. London, 1871.

SHARPE (R. B.). See ' Erebus' \& 'Terror.'

SHARPE (R. B.). Catalogue of the Accipitres, or Diurnal Birds of Prey, in the British Museum. 8\%. London, 1874. See Musedu (British).

SHARPE (R. B.). Catalogue of the Striges, or Nocturnal Birds of Prey, in the British Museum. $8^{\circ}$. London, 1875. See Musedm (British).

SHARPE (R. B.). Catalogue of the Passeriformes, or Perching Birds, in the British Museum.

[In Progress.] See MIuseum (British).

8. London, 1877-83.

SHARPE (R. B.) \& DRESSER (H. E.). A History of the Birds of Europe.

4. London, 1871-79.

SHAW (G.). Zoological Lectures delivered in the years $1806 \& 1807$.

2 vols. $8^{\circ}$. London, 1809.

SHAW (G.). Museum Leverianum. Containing select Specimens from the Museum of the late Sir Ashton Lever, Kt. ; with Descriptions in Latin and English.

$4^{\circ}$. Lonclon, 1792.

SHAW (G.) \& NODDER (F. P.). The Naturalist's Miscellany. 24 vols. $8^{\circ}$. London, 1789-1813.

SHAW (G.) \& STEPHENS (J. F.). General Zoology, or Systematic Natural History.

28 vols. $8^{\circ}$. London, 1800-26.

SHAW LEFEVRE (Right Hon. G.). See Fisheries: Iondon. International Fisheries Exhibition, 1883.

SHELLEY (G. E.). A Handbook of the Birds of Egypt.

8. London, 1872. 
SHELLEY (G. E.). A Monograph of the Cinnyrida. $4^{\circ}$. London, 1876.

SHIELD (R.). Practical Hints respecting Moths and Butterflies, with notices of their localities.

12. London, 1856.

SHUCKARD (W. E.). A Manual of Entomology, translated from the German of Dr. Herman Buruieister. 8. London, 1836.

SHUCKARD (W. E.). Essay on the indigenous Fossorial Hymenoptera.

8.. London, 1837.

SHUCKARD (W. E.). Elements of British Entomology.

8. London, 1839.

SHUCKARD (W. E.). British Bees. $8^{\circ}$. London, 1866.

SHUTTLEWORTH (R. J.). Notitiæ Malacologicæ.

8.. Bern, 1878.

SIBBALD (R.). Phalainologia Nova ; sive Observationes de Rarioribus quibusdam Balcenis in Scotiæ littus nuper ejectis.

8. Edinburgh, 1773.

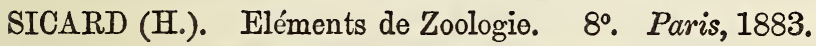

SICHEL (J.). See Saussdre (H. de).

SICHERER (P. F.). Seps tridactylus.

$4^{\circ}$. Tubinga, 1825.

SIDDALL (J. D.) \& BRADY (H. B.). Catalogue of British Recent Foraminifera, for the use of Collectors.

8. Chester, 1879.

SIEBKE (H.) \& SCHNEIDER (J. S.). Enumeratio Insectorum Norvegicorum.

Fasc. 1-3. $8^{\circ}$. Christiania, 1874-76.

SIEBOLD (C. T. von). Observationes quædam de Salamandris et Tritonibus.

40. Berolini, 1828. 
SIEBOLD (C. T. von). Ueber die Band- und Blasenwürmer. 8. Leipzig, 1854.

SIEBOLD (C. T. von). Wahre Parthenogenesis bei Schmetterlingen und Bienen.

8. Leipzig, 1856.

SIEBOLD (C. T. von). On a true Parthenogenesis in Moths and Bees. Translated by W. S. Dallas.

$8^{\circ}$. London, 1857.

SIEBOLD (C. T. von). Die Süsswasserfische von MittelEuropa.

8. Leipzig, 1863.

SIEBOLD (C. T. von). Beiträge zur Parthenogenesis der Arthropoden.

8. Leipzig, 1871.

SIEBOLD (C. T. von). Mittheilungen über die SpeicheIorgane der Biene.

$4^{\circ}$. Nördlingen, 1872.

SIEBOLD (C.T. von). Fauna der Süsswasser-Fische von Mittel-Europa. Mit einer Anleitung zum Bestimmen von F. A. LorI.

8.. Passau, 1878.

SIEBOLD (C. T. von). See Periodicals: Leipzig.

SIEBOLD (C. T. von) \& STANNIUS (H.). Lehrbuch der vergleichenden Anatomie.

I. Wirbellose Thiere, von Siebold.

II. Wirbelthiere, von Stannius.

2 vols. $8^{\circ}$. Berlin, 1846-48.

SIEBOLD (C. T. von) \& STANNIUS (H.). Handbuch der Zootomie. Die Wirbelthiere. $8^{\circ}$. Beriin, 1854-56.

SIEDAMGROTZKY (0.). Ueber die Structur und das Wachsthum der Hornscheiden der Wiederkäuer, und der Krallen der Fleischfresser. $\quad 8^{\circ}$. Dresden, 1871.

SIEGEL (M.). Versuch einer Käfer-Fauna Krains.

8. Laibach, 1866.

SILBE RMANN (G.). Revue Entomologique.

5 vols. $8^{\circ}$. Strusbourg, 1833-37.

SILBERMANN (G.). See Wrincker (J.). 
SIMEON (C.). Stray Notes on Fishing and Natural History. 8. Cambridge \& London, 1860.

SIMON (E.). Histoire naturelle des Araignées (Aranéides). 8.. Paris, 1864.

SIMON (E.). Les Arachnides de France. Tome I.-IV. \& VII. 8 $8^{\circ}$ Puris, 1874-79.

SISMONDA (A. E.). Synopsis Methodica Animalium Invertebratorum Pedemontii Fossilium.

8. Augustce Taurinorum, 1842.

SLABBER (M.). Physikalische Belustigungen oder Mikroskopische Wahrnehmungen von drey und vierzig inund aus-ländischen Wasser- und Land-Thierchen.

$4^{\circ}$. Nürnberg, 1781.

SMELLIE (W.). The Philosophy of Natural History.

$4^{\circ}$. Edinburgh, 1790.

SMITH (Sir A.). Illustrations of the Zoology of South Africa.

$4^{\circ}$. London, 1849.

SMITH (Sir A.). See Periodicals : London. Willughby Society.

SMITH (C.). The Birds of Somersetshire.

post $8^{\circ}$. London, 1869.

SMITH (C. H.). Horses. The Equidce or Genus Equus of Authors.

fol. MS., 1841.

SMITH (C. H.). The Natural History of the Human Species. 8. Edinburgh, 1848.

SMITH (E. A.). See 'Erebus' \& 'Terror.'

SMITH (E. N.). See KueIN (E.) \& Smith (E. N.).

SMITH (F.). Catalogue of British Hymenoptera in the Collection of the British Museum. Part I. Apidæ (Bees).

See Museum (British). $16^{\circ}$. London, 1855 .

SMITH (F.). A Catalogue of British Hymenoptera: Aculeata.

See Musedm (British).

8. London, 1871. 
SMITH (F.). Catalogue of the British Bees in the British Museum. 2nd edition. 80. London, 1876. See Musetur (British).

SMITH (F.). Scientific results of the second Yarkand Mission. Hymenoptera. Seo Survers \& Explorations: Yariand.

SMITH (F.). New species of Hymenoptera in the British MIuseum.

See MIUsedur (British).

SMITH (F. A.). See Fisheries : London.

SMITH (W.). List of British Diatomacece in the British Museum.

See Mrosedr (British).

\section{SMITHSONIAN INSTITUTION.}

Annual Reports of the Smithsonian Institution.

8. Washington, 1849-67.

Catalogue of the described Coleoptera of the United States. By F. E. Mrelshermer.

$8^{\circ}$. Washington, 1853.

Catalogue of North-American Reptiles. By S. F. BAIRD and S. GIRARD. $\quad 8^{\circ}$. Washington, 1853.

List of Birds in the Museum. By S. F. BaIrd. 40. Washington, 1857.

Catalogue of North-American Birds. By S. F. BAIRD. $4^{\circ}$. Washington, 1858.

Directions for collecting and preserving specimens of Natural History.

3rd edition. 8०. Washington, 1859.

Circular in reference to collecting Nests and Eggs of North-American Birds. $\quad$ 80. Washington, 1861.

Arrangement of Families of Birds.

8. Washington, 1866. 


\section{SMITHSONIAN MISCELLANEOUS COLLECTIONS.}

Catalogue of Diptera of North America. By R. OstenSACKEN.

80. Washington, 1858.

Check-Lists of the Shells of North America. By I. Les and P. P. CARPenter. $8^{\circ}$. Washington, 1860.

Synopsis of the Neuroptera of North America. By H. A. HAGEN. $\quad 8^{\circ}$. Washington, 1861 .

Classification of the Coleoptera of North America. Part I. By J. L. Leconte. $8^{\circ}$. Washington, 1861 .

Smithsonian Museum Miscellanea.

8. Washington, 1862.

Monographs of the Diptera of North America. By $\mathbf{H}$. LOEW and R. OsTEN-SACKEN.

Parts I. \& II. 80. Washington, 1862-64.

New species of North-American Coleoptera, by J. L. LeConte. $\quad$ 8. Washington, 1863-66.

List of the Coleoptera of North America. By J. L. LECONTE.

8. Washington, 1863-66.

Catalogue of the Orthoptera of North America. By S. H. SCUDDER. 8.. Washington, 1868.

Arrangement of the Families of Mollusks. By T. Ginc.

80. Washington, 1871.

Arrangement of the Families of Mammals. By T. GiLc.

8. Washington, 1872.

SMITT (F. A.). Skandinaviens Hafs-Bryozoer.

8०. Stockholm, 1868.

SMITT (F. A.). Floridan Bryozoa. Part I.

$8^{\circ}$. Stockholm, 1872.

SMUTS (J.). Dissertatio Zoologica, enumerationem Mammalium Capensium continens. $\quad 4^{\circ}$. Leidoe, 1832.

SNELLEN (P. C. T.). Do Vlinders van Nederland. Macrolepidoptera. roy. 8'. 'S Gravenhage, 1867.

SNELLEN VAN VOLLENHOVEN (S. C.). Natuurlijke historie van Nederland: Overzigt der Gelede Dieren.

$8^{\circ}$. Haarlem, 1860. 
SYELLEN TAN TOLLENHOTEN (S. C.). Beschrijringen en Afbeeldingen ran Nederlandsche Tlinders.

Tol. I. $4^{\circ}$. Amsterdam, 1860. Tol. II. \& III. 'S Gravenhage, 1870-77.

SNELLEN TAN TOLLENHOTEN (S. C.). Faune Entomologique de l'archipel Indo-Néerlandais.

40. La Haye, 1863-68.

SAELLEN TAY TOLIENHOTEN (S. C.). Schetsen ten gebruike bij de studie der Hrmenoptera.

obl. fol. 'S Gravenhage, 1868.

SYELLEN TAN TOLLENHOVEN (S. C.). Pinacographia. $4^{\circ}$. 'S Gravenhage, 1880 .

SNELLEN TAN TOLLENHOTEN (S. C.). See PERIOdicals: ThF. Hagce.

SOLA (Lieut.-Col. F. G.). See Fisheries: Loxdox.

SOLATDEP (D.). The Natural History of Zoophytes collected by the late John Ellis. $4^{\circ}$. London, 1786.

SOLBRIG (A.). Teber die feinere Structur der Nerrenelemente bei den Gasteropoden. 4․ Leipzig, 1872.

SOLIIS-LACBACH (Graf zu). See Fatxa txd Flora des Golfes ron Neapel.

SÖMIMERIYG (S. T.). Teber die körperliche Terschiedenheit des Yegers rom Europäer.

8. Frankfurt-am-Main, 1785 .

SONIET (?). See SAIGI (J. F.).

SOUANCE (C. de), arec le Prince Boraparte et E. Blarchard. Iconographie des Perroquets.

$4^{\circ}$. Paris, 1857.

SOCBEIRAN (J. L.). Études sur l'incubation artificielle. 8. Angers, 1862.

SOTBEIPAT (J. L.). Pisciculture dans les Neilgherries. 8. Paris, 1870.

SOCBEIRAN (J. L.). Curiosités de l'Alimentation.

8. Paris, 1871. 
SOUbeiran (J. L.). See Dabry de Thiersant (P.).

SOUBEIRAN (J. L.). See Fisheries : Havre.

SOULEYET \& EYDOUX. See 'Bontte.'

SOUTHALL (Ј.). A Treatise of Buggs.

8. London, 1730.

SOUTHWELL (T.). The Seals and Whales of the British Seas. 4\%. London, 1881.

SOWERBY (G. B.). A Catalogue of the Shells in the collection of the Earl of Tankerville. $8^{\circ}$. London, 1825.

SOWERBY (G. B.). Malacological and Conchological Magazine.

Part I. (all published). $8^{\circ}$. Privately printed, 1838.

SOWERBY (G. B.). Conchological Illustrations.

8. London, 1841.

SOWERBY (G. B.). Thesaurus Conchyliorum.

Parts I.-XXXVJII. $\quad 8^{\circ}$. London, 1847-82.

SOWERBY (G. B.). Conchological Manual.

8. London, 1852.

SOWERBY (G. B.). Popular History of the Aquarium of Marine and Fresh-water Animals and Plants.

sm. 4․ London, 1877.

SOWERBY (J.). Conchologie minéralogique de la Grande Bretagne.

80. Neuchâtel, 1837.

SOWERBY (J.). British Miscellany. $8^{\circ}$. London, 1804-6.

SOWERBY (J. \& G. B.). Genera of Recent and Fossil Shells. 2 vols. $8^{\circ}$. London, 1820-25.

SOWERBY (J. de C.) \& LEAR (E.). Tortoises, Terrapins, and Turtles. The plates drawn under the superintendence of Thomas Beld. Preface and Notes by J. E. GraY.

$4^{\circ}$. London, 1872. 
SPALLANZANI(L.). Dissertations relative to the Natural History of Animals and Vegetables.

2 rols. $8^{\circ}$. London, 1784.

SPALLANZANI (L.). Viaggi alle due Sicilie e in alcune parti dell' Appennino. 6 vols. $8^{\circ}$. Pavia, 1792-97.

SPALLANZANI (L.). Reisen in beyde Sicilien, und in einige Gegenden der Appenninen.

4 vols. in 2. $8^{\circ}$. Leipzig, 1795-96.

SPALOWSKY (J. J. N. A.). Prodromus in Systema Historicum Testaceorum. fol. Wien, 1795.

SPANGBERG (J.). See PerIodicals: StockHolm.

SPANGENBERG (G.). Disquisitio circa Partes Genitales Foemineas Avium. 4. Göttingae, 1813.

SPEYER (Ad. \& Aug.). Die geographische Verbreitung der Schmetterlinge Deutschlands und der Schweiz.

8.. Leipzig, 1858-62.

SPINOLA (M.). Insectorum Liguriæ species novæ aut rariores. 2 vols. in 1. $4^{\circ}$. Genuce, 1806-8.

SPITOLA (MI.). Essai sur les Insectes Hémiptères, Rhyngotes ou Hétéroptères. $\quad 8^{\circ}$. Paris, 1840.

SPINOLA (MI.). Essai monographique sur les Clérites, Insectes Coléoptères. 8․ Gênes, 1844.

SPIX (J. B.). Geschichte aller Systeme in der Zoologie. 2 vols. $8^{\circ}$. Nürnberg, 1811.

SPIX (J. B.). Cephalogenesis sive capitis ossei structura, formatio et significatio. fol. Monachii, 1815.

SPIX (J. B.). Animalia nova, sive species novæ Lacertarum quas in itinere per Brasiliam collegit J. B. de Spix.

$4^{\circ}$. Monachii, 1825.

SPIX (J. B.). Simiarum et Vespertilionum Brasiliensium species noræ.

fol. Monachii, 1823. 
SPIX (J. B.). Serpentium Brasiliensium species novæ ; ou Histoire Naturelle, écrite par JEAN WAGLER.

4․ Monachii, 1824.

SPIX (J. B.). Avium species novæ.

2 vols. $4^{\circ}$. Monachii, 1824-25.

SPIX (J. B.). Testacea fluviatilia, descripsit Dr. J. A. Wagner. Edit. F. a P. Schrank et C. F. P. De Martius.

fol. Monachii, 1828.

SPIX (J. B.). Species Noræ Testudinum.

fol. Monachii, 1840.

SPIX (J. B.) \& AGASSIZ (L.). Selecta genera et species Piscium.

fol. Monachii, 1829.

SPIX (J. B.) \& MARTIUS (C. F. P.). Delectus Animalium Articulatorum. Descripsit Max. Perty.

$4^{\circ}$. Monachii, 1830-34.

SPRENGEL (W. \& K.). See Cavolint (P.).

SPRY (W.) \& SHUCKARD (W. E.). The British Coleoptera delineated.

8. London, 1840.

STABILE (J.). Fossiles des environs du lac de Lugano. 12. Lugan, 1861.

STABILE(J.). Mollusques Terrestres vivants du Piémont. 8. Milan, 1864.

STAINTON (H. T.). An attempt at a Systematic Catalogue of the British Tineidce and Pterophoridce.

8. London, 1849.

STAINTON (H. T.). The Entomologist's Companion. 2nd edition. $12^{\circ}$. London, 1854.

STAINTON (H. T.). Insecta Britannica. Vol. III. Lepidoptera : Tineina. $\quad 8^{\circ}$. London, 1854.

STAINTON (H. T.). A Manual of British Butterflies and Moths. 2 vols. $12^{\circ}$. London, 1857-59. 
STAINTON (H. T.). The Natural History of the Tineina. 10 vols. $8^{\circ}$. London, 1855-67.

STAINTON (H. T.). The Tineina of Syria and Asia Minor. $8^{\circ}$. Londoñ, 1867.

STAINTON (H. T.). The Tineina of Southern Europe.

8. London, 1869.

STaINTON (H. T.). See Periodicals : London.

STAINTON (H. T.). See Clemens (B.).

STÅL (C.). Monographie des Chrysomélides de l'Amérique. 4․ Upsal, 1863.

STÅL (C.). See 'EUGENIE.'

STANGE (A.). Verzeichniss der Schmetterlinge der Umgegend ron Halle an der Saale. $\quad 8^{\circ}$. Leipzig, 1869.

STANLEY (E.). A Familiar History of Birds.

6th edition. $12^{\circ}$. London, 1854.

STANNIUS (H.). Symbolæ ad Anatomiam Piscium.

$4^{\circ}$. Rostochii, 1839.

STANNIUS (H.). Beiträge zur Kenntniss der Amerikanischen Manati's.

$4^{\circ}$. Rostock, 1846.

STANNIUS (H.). Ueber das Verhältniss der Ganoïden zu den Clupeiden, insbesondere zu Butirinus.

8.. Rostock, 1846.

STANNIUS (H.). Das peripherische Nervensystem der Fische. 80. Rostock, 1849.

STANNIUS (H.). Beobachtungen über Verjüngungsvorgänge im thierschen Organismus.

8. Rostock \& Schwerin, 1853.

STANNIUS (H.). See Siebold (C. T. von).

STARK (J.). Elements of Natural History.

2 vols. $8^{\circ}$. Edinburgh \& London, 1828. 
STARK (J.). On the existence of an Osseous Structure in the Vertebral Column of Cartilaginous Fishes.

$4^{\circ}$. Edinburgh, 1844.

STAUB (J.). Die Pfahlbauten in den Schweizer Seen.

8. Züirich, 1864.

STAUDINGER (0.). De Sesiis Agri Berolinensis.

$4^{\circ}$. Berolini, 1854.

STAUDINGER (O.) \& WOCKE (M.). Catalog der Lepidopteren des Europäischen Faunengebiets.

2nd edition. $8^{\circ}$. Dresclen, 1871.

STAVELEY (E. F.). British Spiders. $\quad 8^{\circ}$. London, $1866^{\circ}$.

STAVELEY (E. F.). British Insects. 80. London, 1871.

STEEDMAN (A.). Wanderings and Adventures in the Interior of Southern Africa. 2 vols. $8^{\circ}$. London, 1835.

STEEG (G.). De Anatomia et Morphologia squamarum Piscium. 2nd edition. 8. Bonnce, 1857.

STEENSTRUP (J. J. S.). Ueber den Generationswechsel. Ed. LoRENzen. $8^{\circ}$. Copenhagen, 1842.

STEENSTRUP (J. J. S.). Untersuchungen über das Vorkommen des Hermaphroditismus in der Natur. Aus dem Dänischen ïbersetzt von C. F. HoRsschucr.

$4^{\circ} . \quad$ Greifswald, 1846.

STEENSTRUP (J. J. S.). See Ray Societr.

STEFFAHNY (G. A.). Tentamen Monographiæ Byrrhorum Coleopterorum generis. $\quad 8^{\circ}$. Berolini, 1842.

STEFFEN (G. A.). De Ranis nonnullis observationes anatomicæ. 4. Berolini, 1815.

STEIN (F.). Vergleichende Anatomie und Physiologie der Insecten. fol. Berlin, 1847.

STEIN (F.). Der Organismus der Infusionsthiere. Abth. I.-III. $4^{\circ}$. Leipzig, 1859-78. 
STEIN (J. P. E.). Die lebenden Schnecken und Muscheln der Umgegend Berlins.

$8^{\circ}$. Berlin, 1850.

STEIN (J. P. E.) \& WEISE (J.). Catalogi Coleopterorum Europæ editio secunda.

8. Berlin, 1877.

STEINBUCH (J. G.). Commentatio de Tænia hydatigena anomala, adnexis cogitatis quibusdam de vermium visceralium physiologia.

8. Erlanga, 1802.

STEINDACHNER (F.). Beiträge zur Kenntniss der Gobioiden. 8. Wien, 1860.

STEINDACHNER (F.). Ichthyologische Mittheilungen. Parts IV., VI., VIII., IX. $\quad 8^{\circ}$. Wien, 1862-66.

STEINDACHNER (F.). Ueber eine neue Epicrates-Art aus Columbien. 4\%. Wien, 1863.

STEINDACHNER (F.). Beiträge zur Kenntniss der Sciænoiden Brasiliens und der Cyprinodonten Mejicos.

8. Wien, 1863.

STEINDACHNER (F.). Batrachologische Mittheilungen. $8^{\circ}$. Wien, 1864.

STEINDACHNER (F.). Beiträge zur Kentniss der Chromiden Mejicos und Central-America's. $4^{\circ}$. Wien, 1864.

STEINDACHNER (F.). Catalogue préliminaire des Poissons d'eau douce de Portugal. Conservés au Muséum d'Histoire Naturelle de Lisbonne. $\quad 4^{\circ}$. Lisbonne, 1864.

STEINDACHNER (F.). Ichthyologische Notizen.

I. 1864, II. 1865, III. 1866, IV.-VI. 1867, VII. 1868, VIII.-IX. 1869, X. 1870. $\quad 8^{\circ}$. Wien, 1864-70.

STEINDACHNER (F.). Ueber einige neue und seltene Meeresfische aus China. $\quad 8^{\circ}$. Wien, 1865.

STEINDACHNER (F.). Ichthylogischer Bericht über eine nach Spanien und Portugal unternommene Reise.

I.-VI. $8^{\circ}$. Wien, 1865-68. 
STEINDACHNER (F.). Zur Flussfischfauna von Croatien. 8. Wien, 1865.

STEINDACHNER (F.). Zur Fischfauna von Port Jackson in Australien.

8. Wien, 1866.

STEINDACHNER (F.). Ueber eine neue Mustelus-Art von Port Natal.

8․ Wien, 1866.

STEINDACHNER (F.). Ueber Cephalus hypophthalmus. 8․ Wien, 1866.

STEINDACHNER (F.). Ueber Barbus mayori, Val., und Lota vulgaris, Cuv. 8. Wien, 1866.

STEINDACHNER (F.). Herpetologische Notizen.

8. Wien, 1867-78.

STEINDACHNER (F.). Ueber einige Fische aus dem Fitzroy-Flusse bei Rockhampton in Ost-Australien.

8. Wien, 1867.

STEINDACHNER (F.). Ueber einige Meeresfische aus der Umgebung von Monravia in West-Afrika.

8․ Wien, 1867.

STEINDACHNER (F.). Ueber eine neue PlecostomusArt aus Brasilien. 8․ Wien, 1867.

STEINDACHNER (F.). Polypterus lapradei, sp. n., und $P$. senegalus, Cur., aus dem Senegal. $\quad 8^{\circ}$. Wien, 1867.

STEINDACHNER (F.). Die Gymnotidæ des k. k. HofNaturaliencabinets zu Wien.

8.. Wien, 1868.

STEINDACHNER (F.). Zur Fischfauna des Senegal. I.-III. $4^{\circ}$. Wien, 1869-70.

STEINDACHNER (F.). Uber eine neue Gattung und Art aus der Familie der Pleuronectiden und über eine neue Thymallus-Art. 8. Wien, 1874.

STEINDACHNER (F.). Ichthyologische Beiträge. I.VIII. 8. Wien, 1871-79. 
STEINDACHNER (F.). Die Süsswasserfische des südöstlichen Brasilien.

STEINDACHNER (F.). Ueber einige neue brasilianische Siluroiden aus der Gruppe der Doradinen.

8.. Wien, 1875.

STELYDACHNER (F.). Beiträge zur Kenntniss der Chromiden des Amazonstromes.

$8^{\circ}$. Wien, 1875.

STEINDACHNER (F.). Die Schlangen und Eidechsen der Galapagos-Inseln.

$4^{\circ}$. Wien, 1876.

STEINDACHNER (F.). Über zwei neue EidechsenArten aus Süd-Amerika und Borneo. $\quad 4^{\circ}$. Wien, 1877.

STEINDACHNER (F.). Zur Fisch-Fauna des MagdalenenStromes.

$4^{\circ}$. Wien, 1878.

STEINDACHNER (F.). Ueber einige neue und seltene Fisch-Arten aus den k. k. zoologischen Museen zu Wien, Stuttgart und Warschau. 4\%. Wien, 1879 .

STEINDACHNER (F.). Zur Fisch-Fauna des Cauca und der Flïsse bei Guayaquil. $\quad 8^{\circ}$. Wien, 1880.

STEINDACHNER (F.). Beiträge zur Kenntniss der Flussfische Südamerika's. I.-III. $\quad 4^{\circ}$. Wien, 1881.

STEINDACHNER (F.). Batrachologische Beiträge.

8. Wien, 1882.

STEINDACHNER (F.). Beiträge zur Kenntniss der Fische Afrika's und Beschreibung einer neuen Sargus-Art von den Galapagos-Inseln. $\quad 4^{\circ}$. Wien, 1882.

STEINDACHNER (F.). Beiträge zur Kenntniss der Fische Afrika's (II.) und Beschreibung einer neuen Paraphoxinus Art aus der Herzegowina. $\quad 4^{\circ}$. Wien, 1882.

STEINDACHNER (F.). See 'NotaRa.'

STEINHEIM (S. L.). Die Entwickelung der Frösche. Ein Beitrag zur Lehre der Epiginese. 80. Hamburg, 1820. 
STELLER (G. W.). Beschreibung ron Sonderbaren Meerthieren. $\quad 8^{\circ}$. Halle, 1753.

STELLER (G. W.). Beschreibung von dem Lande Kamtschatka.

$8^{\circ}$. Frankfurt \& Leipzig, 1774.

STEPHANOF (P.). Ueber Geschlechtsorgane und Entwickelung von Ancylus fluviatilis.

$4^{\circ}$. St. Petersburg, 1866.

STEPHENS (J. F.). A Systematic Catalogue of British Insects.

8. London, 1829.

STEPHENS (J. F.). Nomenclature of British Insects.

2nd edition. $8^{\circ}$. London, 1833.

STEPHENS (J. F.). Manual of British Coleoptera or Beetles.

8. ${ }^{\circ}$ London, 1839.

STEPHENS (J. F.). Illustrations of British Entomology. 12 vols. $8^{\circ}$. London, 1828-46

STEPHENS (J. F.). Bibliotheea Stephensiana.

$4^{\circ}$. London, 1853.

STEPHENS (J. F.). See Shaw (G.).

STERLAND (W. J.). The Birds of Sherwood Forest.

8.. London, 1869.

[STETTIN.] Catalogus Coleopterorum Europæ.

5th \& 6th editions. $8^{\circ}$. Stettin, 1855-56.

STEVENSON (H.). The Birds of Norfolk.

2 vols. 80. London, 1866-70.

STEWART (W. C.). The Practical Angler.

12. Edinbwrgh, 1857.

STIEBEL (Dr.). See Periodicals : Frankfurt a. M.

STIEDA (L.). Teber das Rückenmark und einzelne Theile des Gehirns von Esox lucius, L.

$4^{\circ}$. Dorpat, 1861. 
STIEDA (L.). Die Bildung des Knochengewebes.

$4^{\circ}$. Leipzig, 1872.

STIEDA (L.). Studien über den Amphioxus lanceolatus.

$4^{\circ}$. St. Petersburg, 1873.

STIEDA (L.). Karl Ernst von Baer. Eine biographische Skizze.

8. Braunschweig, 1878.

STIERLIN (G.). Revision der Europäischen Otiorhynchus-Arten.

8. Berlin, 1861.

STIERLIN (G.) \& GAUTARD (V.). Die Käfer-Fauna der Schweiz.

4. Schaffhausen, 1867.

STIMPSON (W.). Shells of New England.

8. Boston, 1851.

STIMPSON (W.). Synopsis of the Marine Invertebrata of Grand Manan. $4^{\circ}$. Washington, 1853-54.

STIMPSON (W.). The Crustacea and Echinodermata of the Pacific shores of North America.

8. Riverside, Cambridge, U.S., 1857.

STIMPSON (W.). Notes on North-American Crustacea.

8. New York, 1859.

STOLICZKA(F.). See Strvitys \&ExploRations : Yarkand.

STOLL (C.). Natuurlyke en naar 't leeven naauwkeurig gekleurde Afbeeldingen en Beschryvingen der Cicaden en der Wantzen.

Représentation exactement coloriée d'après nature des Cigales et des Punaises. $\quad 4^{\circ}$. Amsterdam, 1788.

STOLL (C.). See Cramer (P.).

STORER (D. H.). A Synopsis of the Fishes of North America. $4^{\circ}$. Cambridge, 1846.

STORER (D. H.). Seo Surveys \& Explorations: Boston. 
STORER (J.). The Wild White Cattle of Great Britain.

8. London, 1881.

STRANGE (G.). See OLIVI (G.).

STRASSER (H.). Zur Lehre von der Ortsbewegung der Fische.

8. Stuttgart, 1882.

STRAUCH (A.). De Loco Monotrematibus in Systemate Zoologico assignando, et de Ornithorhynchi anatini, Shaw, Calcari.

8. Dorpati Livonorum, 18:9.

STRAUCH (A.). Catalogue systématique de tous les Coléoptères décrits dans les Annales de la Soc. Ent. de France (1832-1859).

$8^{\circ}$. Halle, 1861.

STRAUCH (A.). Essai d'une Erpétologie de l'Algérie. $4^{\circ}$. St. Petersburg, 1862.

STRAUCH (A.). Chelonologische Studien.

$4^{\circ}$. St. Peiersburg, 1862.

STRAUCH (A.). Synopsis der Viperiden.

$4^{\circ}$. St. Petersburg, 1869.

STRAUCH (A.). Revision der Salamandriden-Gattungen. $4^{\circ}$. St. Petersburg, 1870.

STRAUCH (A.). Die Schlangen des Russischen Reichs.

$4^{\circ}$. St. Petersburg, 1873.

STRAUS-DURCKHEIM (H.). Considérations générales sur l'anatomie eomparée des Animaux Articulés.

$4^{\circ}$. Paris, 1828.

STRAUS-DURCKHEIM (H.). Anatomie descriptive et comparative du Chat.

Text, 2 vols. $4^{\circ}$. Atlas, fol. Paris, 1845.

STREBEL (H.). Beitrag zur Kenntniss der Fauna Mexikanischer I and- und Süsswasser-Conchylien.

Theile I.-V. $4^{\circ}$. Hamburg, 1873-82. 
STRECKER (H.). Lepidoptera.

I5 parts. $4^{\circ}$. Reading, U.S., 1872-77.

STRECKER (H.). Butterflies and Moths of North America. Diurnes. $\quad 8^{\circ}$. Reading, U.S., 1878.

STRETCH (R, H.). See REdoRTs : WaSHINGtoN, U. S.

STRETCH (R. H.). See Surveys \& Explorations: United States. Washington.

STRICKER (S.). Handbuch der Lehre von den Geweben des Menschen und der Thiere.

2 vols. in 1. $8^{\circ}$. Leipzig, 1871-72.

STRICKLAND (H. E.). Ornithological Synonyms. Edited by Sir Willialr JaRdine, Bart.

Vol. 1. Accipitres. $8^{\circ}$. London, 1855.

STRICKLAND (H. E.), Nemoirs of. By Sir WrLLIAZE

JARDINE, Bart. 8.. London, 1858.

STRICKLAND (H. E.). See SALtiN (O.).

STRICKLAND (H. E.) \& MELVILLE (A. G.). The Dodo and its Kindred. $4^{\circ}$. London, 1848.

STROBEL (P. de). Delle Conchiglie terrestri dei dintorni d' Innsbruck.

$8^{\circ}$. 1844.

STUDER (J. H.). Popular Ornithology. The Birds of North America. 4.. New York, n. d.

STUDER (T.). Uebersicht der Steinkorallen. Madreporia, Eupsammina, Turbinarina. $\quad 8^{\circ}$. Berlin, 1877.

STUDER (T.). Uebersicht der Antrozoa alcyonaria.

$8^{\circ}$. Berlin, 1878.

STURM (J.). Insecten Cabinet.

12․ Nürnberg, 1791-92.

STURM (J.). Verzeichniss meiner Insecten Sammlung.

12․ Nürnberg, 1796. 
STURM (J.). Abbildungen zu Karl Illiger's Uebersetzung von Olivier's Entomologie; oder Naturgeschichte der Insecten mit ihren Gattungs- und Artmerkmalen, ihrer Beschreibung und Synonymie. Käfer.

2 vols. text, and 1 vol. plates. $4^{\circ}$. Nürnberg, 1802-3.

STURM (J.). Verzeichniss meiner Insecten-Sammlung.

8. Nürnberg, 1800.

STURM (J.). Verzeichniss einiger zum Tausch vorräthiger Insecten.

8. Nürnberg, 1804.

STURM (J.). Deutschlands Fauna.

8 vols. $8^{\circ}$. Nürnberg, 1805-47.

STURM (J.). Catalog meiner Insecten-Sammlung. Käfer. 8. Nürnberg, 1826.

STURM (J.). Catalog der Käfer-Sammlung.

8․ Niirnberg, 1843.

SUCKLEY $\left(G_{0}\right)$. See Strveys \& Explerations: United Strates. New York.

SUCKLEY (G.) \& COOPER (J. G.). The Natural History of Washington Territory and Oregon. 4. New York, 1860.

SUCKOW (F. W. L.). Myologiæ Insectorum specimen de Astaco fluviatili.

$4^{\circ}$. Heidelberga, 1813.

SUCKOW (F. W. L.). Anatomisch-Physiologische Untersuchungen der Insekten und Krustenthiere.

$4^{\circ}$. Heidlelberg, 1818.

SUCQUET (J. P.). D'une Circulation du Sang spéciale au rein des Animaux vertébrés. $\quad 8^{\circ}$. Paris, 1867.

SULZER (F. G.). Versuch einer Naturgeschichte des Hamsters. $12^{\circ}$. Gottingen \& Gotha, 1774 .

SULZER (J. H.). Abgekürzte Geschichte der Insecten. $4^{\circ}$. Winterthur, 1776.

SUMICHRAST (F.). The Geographical Distribution of the Native Birds of Vera Cruz. $\quad 4^{\circ}$. Boston, 1869. 
SUNDEVALL (C.). Methodische Uebersicht der Wiederkauenden Thiere (Pecora). (Uebersetzung von HorNsCHUCH.)

$8^{\circ}$. Greifswald, 1848.

SUNDEVALL (C. J.). Srenska Foglarna.

obl. fol. Stockholm, 1856.

SUNDEVALL (C. J.). Die Thierarten des Aristoteles.

8. Stockholm, 1863.

SUNDEVALL (C. J.). Conspectum Avium Picinarum edidit C. J. S. 8 . Stockholmice, 1866.

SUNDEVALL (C. J.). Methodi naturalis Avium disponendorum Tentamen.

80. Stockholm, 1872.

SUNDEVALL (C. J.). Skandinaviens Fiskar. See Fries (B. T.).

SURINGAR (G. C. B.). Catalogus Craniorum.

$8^{\circ}$. Lugduni Batavorum, 1876.

SURVEYS AND EXPLORATIONS.

Fratce.

Exploration Scientifique de l'Algérie, 1840-42. Zoologie. imp. $4^{\circ}$. Paris, 1848-57.

Crustacés, Arachnides, Myriapodes, et Hexapodes par H. LuCas.

Insectes par H. LucAs.

1849.

Mollusques par G. P. Deshayes. 1848.

Reptiles par - Guichenот. 1850.

Oiseaux par - Loche. 1857.

Mammifères par - LocHe. 1857.

Mission Scientifique en Mexique et dans l'Amérique Centrale, ouvrage publié par ordre de S. M. L'EMPEREUR. 40. Paris, $1870-83$.

Reptiles et Batraciens par A. Duméril et Bocourt. $1870-83$.

Poissons par Léon Vailuant et Bocourt. 1874.

Xiphosaurus et Crustacés par A. Milne-Edwards.

Orthoptères par H. DE SAUSSURE. 1873. 1870 .

Myriapodes par H. DE Saussure et A. HundBert.

1872.

Mollusques par P. Fischer et H. Crosse. 1870-78. Voyage d'Exploration sur le littoral de la France et de l'Italie par M. Coste. $4^{\circ}$. Paris, 1861. 
Germany.

Die Preussische Expedition nach Ost-Asien. Zoologische Abtheilung bearbeitet von E. v. Martens.

2 vols. roy. $8^{\circ}$. Berlin, 1867-76.

Reisen im Archipel der Philippinen von Dr. C. Semper.

$4^{\circ}$. Leipzig \& Wiesbaden, 1868-83.

I. Holothurien von C. Semper.

1868.

II. Malacologische Untersuchungen. Heft. I.XIV. und Suppl. I., II. von R. Bekgh.

$1870-81$.

III. Landmollusken von C. SEMPER. 1870-82.

IV. Sipunculiden I. von E. Selenka. 1883.

[In progress.]

\section{Norifay.}

Norwegian North Atlantic Expedition, 1876-82. (Zoology.)

Fishes. By R. Colietr.

$4^{\circ}$. Christiania.

Gephyrea. By D. C. Danielssen \& J. Koren. 1881.

Holothurioidea. By D. C. Danierssen \& J. KoRen.

Annelida. By G. A. Haxsen.

1882.

Mollusca. By H. Friele.

1882.

1882.

[In progress.]

\section{Rossta.}

Reise in den äussersten Norden und Osten Sibiriens, 1843 und 1844. Von A. T. von Middendorfy. Band II. Zoologie. $\quad 4^{\circ}$. St. Petersburg, 1851-53.

Annulaten von E. Grobe.

Echinodermen von F. Brandt und E. Grube.

Insecten von F. MENÉtrifs.

Hymenopteren und Neuropteren von W.F. Erichson.

Krebse von F. Brandt.

Brachiopoden und Entomostraceen von S. Fisoner.

Mollusken von A. Th. v. Middendorf.

Parasiten von E. GRUBE.

Säugethiere, Vögel und Amphibien von A. Tн. v. Middendorf.

Band IV. i. ii. iii. Uebersicht der Natur Nord und Ost Sibiriens. 1867-75.

United States.

Boston. Reports of the Commissioners on the Zoological Survey of Massachusetts.

Fishes. By D. H. Storer.

Reptiles. By D. H. Storer.

Birds. By W. O. B. Peabody. $\quad 8^{\circ}$. Boston, 1838. 
SURVEYS AND EXPLORATIONS, continued.

New York. The Natural History of Washington Territory and Oregon. By G. SuckreeY and J. G. Cooper.

$4^{\circ}$. New York, 1860.

Philadelphia. United States Exploring Expedition, under the Command of Charles Wilkes, 1838-42.

Mammalogy \& Ornithology, by J. Cیssix. 1858. Herpetology. By C. GiraRd.

1858.

Mollusca. By A. A. Gould.

$1852-56$.

Zoophytes. By J. D. Dana.

$1848-49$.

Crustacea. By J. D. Dana.

$1852-55$.

Text, $4^{\circ}$. Atlas, fol. Philadelphia, 1848-58.

Washington. United States Pacific Railway Route, 47 th and 49th parallels. Explorations and Surveys. Zoological Report.

Insects. By J.'T. Leconte. $4^{\circ}$. Washington, 1860.

Washington. Preliminary Report of the Geological Survey of Montana and portions of adjacent Territories. By F. V. HAYDEN. $8^{\circ}$. Washington, 1872.

Washington. Final Report of the Geological Surrey of Nebraska. By F. V. HAYDEN.

8. Washington, 1872 .

Washington. Report ipon Explorations and Surveys west of the 100th meridian, in charge of Lieut. G. M. Wheeler. Vol. V. Zoology. 4\%. Washington, 1875.

Mammals. By H. C. Yarrow \& E. Coves.

Ornithology. By H. M. Henshaw.

Reptiles and Batrachians. By E. Cooes \& D. C. X ARROW.

Fishes. By E. D. Cope \& H. C. YarRow.

Hymenoptera. By E. T. Cresson.

Formicidæ. By E. Norton.

Lopidoptera (Diurnal), By T. L. Me.sd \& W. H. EDWARDS.

Zygænidæ and Bombycidæ. By R. H. Stretce.

Diptera. By C. Osten-SACKeN.

Coleoptera. By H. UцKe.

Hemiptera. By P. R. UHLER.

Orthoptera. By C. Thomas.

Neuroptera. By H. A. Hagerv.

Mollusca. By T. C. YaRrow.

Freshwater Leeches. By A. E. Verrili.

Washington. 1st and 2nd Annual Reports of the U.S. Entomological Commission for the years 1877-79, relating to the Rocky Mountain Locust. By C. V. Riley, A. S. Packard, jun., \& C. Thomas.

$8^{\circ}$. Washington, 1878-80. 
SURVEYS AND EXPLORATIONS, continued.

Washington. Report of the United States Geological Survey of the Territories : F. V. HAYDEN in charge.

Vol. X. Geometrid Moths. By A. S. Packard. $4^{\circ}$. Washington, 1876.

Vol. XI. North American Rodentia. By E. Codes \& J. A. Allen. $\quad 4^{\circ}$. Washington, 1877. Vol. XII. Freshwater Rhizopods. By J. Leidy.

$4^{\circ}$. Washington, 1879.

Washington. Geological Survey of the Territories: F. V. Haydex in charge. Miscellaneous Publications. $8^{\circ}$. Washington, 1874-80.

No. 3. Birds of the North West. By E. Coues. 1874. Reports on the Zoological Collections of Lieut. W. L. CARPENTER made in Colorado in $1873 . \quad 1875$.

No. 7. Ethnography and Philology of the Hidasta Indians. By W. Matriews. 1877.

No. 8. Fur-bearing Animals. By E. Cours. 1877.

No. 12. North American Pinnipeds. By J. A. AlLex.

1880.

\section{YARKAND.}

Scientific Results of the second Yarkand Mission, based upon the Collections and Notes of the late F. SToLICZKA. $4^{\text {o. }}$ Calcutta, 1878-79.

Geology. By W. T. Buanford.

Mammalia. By W. T. Blanford.

1878.

Chiroptera. By G. E. Dobson.

1879. Reptilia and Amphibia. By W.T. Blanford. 1878. Ichthyology. By F. DAY.

Mollusca. By G. Nevill. 1878.

Hymenoptera. By F. Sигтн.

1878.

Neuroptera. By R. M'Lachlan.

1878.

Lepidoptera. By F. Moore.

1878.

Rhyncota. By W. L. Distant.

Rhizopoda (Syringosphceridce). By P. M. Duncan.

1879 .

YunNan.

Zoological Results of the two Expeditions to Western Yunnan in 1868 and 1875. By JoHN ANderson.

2 vols. $4^{\circ}$. London, 1878.

SVENSK ZOOLOGI ; författad af C. QuenseL, med illuminerade figurer tecknade af J. W. Palnstruch.

Heft. 1-6. 8 . Stockholm, 1806-08. Andra Bandet, utgifven af J. W. Palmstrude, G. J. Billberg, med text författad af O. Swartz.

Heft. 7-12. 8․ Stockholm, 1809-25. 
SWAINSON (W.). Zoological Illustrations.

1st series. 3 vols. $8^{\circ}$. London, 1820-23.

2nd series. 3 vols. $8^{\circ}$. London, 1829-33.

SWAINSON (W.). The Elements of Modern Conchology.

$12^{\circ}$. London, 1835.

SWAINSON (W.). A Treatise on the Geography and Classification of Animals. (Lardner's Cabinet Cyclopædia.)

$12^{\circ}$. London, 1835.

SWAINSON (W.). On the Natural History and Classification of Quadrupeds. (Lardner's Cabinet Cyclopædia.)

12. London, 1835.

SWAINSON (W.). On the Natural History and Classification of Birds.

2 vols. $12^{\circ}$. London, 1836.

SWAINSON (W.). Birds of Western Africa.

12. Edinburgh, 1837.

SWAITSON (W.). Natural History of Fishes, Amphibians and Reptiles. $\quad 2$ vols. $12^{\circ}$. London, 1838-39.

SWAINSON (W.). Two centuries and a quarter of Birds. 12. London, 1838.

SWAINSON (W.). Taxidermy: Bibliography and Biography.

$12^{\circ}$. London, 1840.

SWAINSON (W.). A Treatise on Malacology.

12. London, 1840.

SWAINSON (W.). Exotic Conchology. Second edition, by Sylvands Hanley.

$4^{\circ}$. London, 1841.

SWAINSON (W.). A selection of the Birds of Brazil and Mexico.

8. London, 1841.

SWAINSON (W.) \& SHUCKARD (W. E.). On the History and Natural Arrangement of Insects.

8. London, 1840.

SWALLOW. Observations on the Brumal Retreat of the Swallow. By Philochelidon. $\quad 8^{\circ}$. London, 1808. 
SWAMMERDAM (J.). Historia Insectorum Generalis, ex Belgica Latinam fecit H. C. Henninios.

4\%. Lugduni Batavorum, 1685.

SWAMIERDAM (J.). Historia Insectorum Generalis.

4․ Lugduni Batavorum, 1733.

SWAMMERDAII (J.). Biblia Naturæ; sive Historia Insectorum. 3 vols. fol. Leyda, 1737-38.

SWAMIERDAM (J.). Bibel der Natur, worinnen die Insekten in gewisse Classen vertheilt \&c.

fol. Leipzig, 1752.

SWAMMERDAM (J.). The Book of Nature, or the History of Insects. fol. London, 1758.

SWARTZ (O.). See Svensk Zoologr.

SWEET (R.). The British Warblers, an account of the genus Sylvia. $\quad 8^{\circ}$. London, 1823.

SWINTON (A. H.). Insect Variety : its Propagation and Distribution. $\quad 8^{\circ}$. London, Paris, \& New York, n. d.

SYKES (W. H.). A Catalogue of the Mammalia observed in Dukhun, East Indies. 8.. London, 1831.

SYME (P.). A Treatise on British Song-Birds.

8.. Edinburgh \& London, 1823.

T.

TACZANOWSKI (W.). Oologia Ptakow Polskich. 2 vols. $8^{\circ}$. Warszawa, 1862.

TAit (P. G.). See 'Challenger.'

TAPPARONE CANEFRI (C.). Faune Malacologique de l’île Maurice. 8. Bruxelles, 1881. 
TARDIF (G.). Le Livre de l'art de Faulconnerie et de Chiens de Chasse. 2 vols. in $1.12^{\circ}$. Paris, 1882.

TARENETZKY (A.). Anatomie des Darmkanals.

$4^{\circ}$. St. Petersburg, 1881.

TARNIER (F.). Coléoptères des Iles Açores.

8. Dijon, 1860.

TARNIER (F.). Catalogue des Coquilles terrestres et fluviatiles. 4. Dijon, 1862.

TASCHENBERG (E. L.). Bilder aus dem InsektenLeben. 8. Berlin, 1861.

TASCHENBERG (E. L.). Naturgeschichte der Wirbellosen Thiere.

8. Leipzig, 1865.

TASCHENBERG (E. L.). Die Hymenopteren Deutschlands. 8.. Leipzig, 1866.

TASCHENBERG (E. L.). Entomologie für Gärtner und Gartenfreunde.

8. Leipzig, 1871.

TASCHENBERG (E. L.). Forstwirthschaftliche InsektenKunde. 8. Leipzig, 1874.

TASCHENBERG (E. L.). Praktische Insekten-Kunde oder Naturgeschichte aller derjenigen Insekten, \&c.

$8^{\circ}$. Bremen, 1879-80.

TASCHENBERG (E. O.). Anatomie der Cylicozoa, einer Ordnung der Hydrozoa.

8. Halle, 1877.

TASCHENBERG (O.). Die Flöhe. $\quad 4^{\circ}$. Halle, 1880.

TASCHENBERG (O.). Was sind Holzwürmer und Wie erwehrt man sich ihrer?

8. Halle, 1883.

TAXIDERMY. Taxidermy; or, the Art of Collecting, Preparing, and Mounting Objects of Natural History for the use of Museums and Travellers.

4th edition. $12^{\circ}$. London, 1829. 
TAYlOR (T.). See Mtosedu: College of Surgeons.

TEATINO (P. D. P. Cultrera). Fauna Biblica, orvero Spiegazione degli Animali menzionati nella Sacra Scrittura. 8. Palermo, 1880.

TEGETMEIER (IV. B.). Pigeons. Illustrated by HARRISON WEIR. roy. $8^{\circ}$. London, 1868.

TEGETMEIER (W. B.). Pheasants; their Natural History and Practical Management.

2nd edition. $4^{\circ}$. London, 1881.

TEGETMEIER (W. B.). See BLyth (E.).

TEGETMEIER (W. B.). See Boddaert (P.).

TEGETMEIER (W. B.). See Moore (J.).

TEILMANN (C.). Forfog til en Beskrivelse af Danmarks og Islands Fugle.

$12^{\circ}$. Ribe, 1823.

TEMIMINCK (C. J.). Catalogue systematique du cabinet d'Ornithologie et des Quadrumanes. $8^{\circ}$. Amsterdam, 1807.

TEMMINCK (C. J.). Les Pigeons dessinés par Madame Knip.

fol. Paris, 1811.

TEMIINCK (C. J.). Histoire naturelle des Pigeons et des Gallinacés. 3 vols. $8^{\circ}$. Amsterdam, 1813.

TEMMINCK (C. J.). Manuel d'Ornithologie. 2nd edition. 4 vols. $8^{\circ}$. Paris, 1820 .

TEMMINCK (C. J.). Monographies de Mammologie. 2 vols. $4^{\circ}$. Paris, 1827. Another copy. 2 vols. in 1. $4^{\circ}$. Paris, 1827-41.

TEMMINCK (C. J.). Verhandelingen over de Natuurlijke Geschiedenis der Nederlandsche overzeesche bezittingen. (Zoologie.)

fol. Leiden, 1839-44.

TEMIMINCK (C. J.). Esquisses Zoologiques sur la Côte do Guiné. $1^{\mathrm{e}}$ partie. Les Mammifères. $\quad 8^{\circ}$. Leiden, 1853. 
TEMIMLNCK (C. J.). See Siebold (F. H. von).

TEMIMINCK (C. J.). See Vieillot (L. P.).

TEMIMINCK (C. J.) \& LAUGIER DE CHARTROUSE (Baron II.). Noureau recueil de Planches coloriées d'Oiseanx. 5 vols. $4^{\circ}$. Paris, 1838.

TEMPLE (Capt.). See Fismeries: Loxdon. International Fisheries Exhibition, 1883.

TENNENT (Sir J. E.). Sketches of the Natural History of Ceylon.

$8^{\circ}$. London, 1861.

TERVER (M.). Catalogue des Mollusques du nord do l'Afrique.

8. . Paris, 1839.

TEUTSCHE ORNITHOLOGIE. Herausgegeben ron M. B. Borkhausen, J. W. Lichthajimer, C. W. Berker, Lemicke, und Bekker dem Jüngern. 2 rols. fol. Darmstadt, 1800-5.

THEEL (H.). See 'Challexger.'

THEOBALD (W.). Catalogue of Shells in the Museum of the Asiatic Society of Bengal. $\quad 8^{\circ}$. Calcutta, 1860.

See Musedir (Calcutta).

THEOBALD (W.). Catalogue of the Reptiles of British India. 8. Calcutta, 1876.

THIENEMANN (F. A. L.). Die Fortpflanzung der Vögel Europa's mit Abbildung der Eier. $4^{\circ}$. Leipzig, 1825-38.

THIENEMANN (F. A. L.). Fortpflanzungsgeschichte der gesammten Tögel nach dem gegenwärtigen Standpunkte der Wissenschaft.

$4^{\circ}$. Leipzig, 1845-56.

THIENEMANN (F. A. L.). Rhea.

8. Leipzig, 1846-49.

THOMIS (C.). On the Rocky Mountain Locust. 8. Washington, 1878-80.

See Survers \& Explorations: U. S., Washington.

THOMAS (C.). See Reports : Washington, U.S. 
THOMAS (H. S.). The Rod in India.

8. Mangalore, 1873.

THOMPSON (E. P.). The Note-Book of a Naturalist.

8. London, 1845.

THOMPSON (Sir H.). See FIsHERIES: LoNdon. International Fisheries Exhibition, 1883.

THOMPSON (J. V.). Zoological Researches.

Vol. I. Parts 1-5. $8^{\circ}$. Cork, n. d.

THOMPSON (J. V.). Memoir on Pentacrinus europceus.

4. Cork, 1827.

THOMPSON (R.). Report on Insects destructive to Woods and Forests. $\quad$ 8. Allahabad, 1868.

THOMPSON (W.). The Natural History of Ireland.

4 vols. $8^{\circ}$. London, 1849-56.

THOMSON (C. G.). Skandinaviens Insecter, en Handbok i Entomologi, till elementar-läroverkens tjenst.

$8^{\circ}$. Lund, 1862.

THOMSON (C. G.). Skandinaviens Coleoptera.

8 vols. $8^{\circ}$. Lund, 1859-66.

THOMSON (C. G.). See Periodicals : Lund.

THOMSON (C. G.). Hymenoptera Scandinaviæ.

5 vols. 8 . Lund, 1871-78.

THOMSON (C. G.). See 'EUGENIE.'

THOMSON (C. W.). The Depths of the Sea.

8o London, 1873.

THOMSON (C. W.). The Voyage of the 'Challenger.'

2 vols. $8^{\circ}$. London, 1877.

THOMSON (J.). Archives Entomologiques.

2 vols. $8^{\circ}$. Paris, 1857. 
THOMSON (J.). Monographie des Cicindelides. $4^{\circ}$. Paris, 1857.

THOMSON (J.). Arcana Naturæ. fol. Paris, 1859.

THOMSON (J.). Classification de la famille des Cérambycides. 8. Paris, 1860.

THOMSON (J.). Systema Cerambycidarum.

8. Liége, 1864.

THOMSON (J.). Physis : Recneil d'histoire naturelle. Tom. I. roy. $8^{\circ}$. Paris, 1867. [All published.]

THON (Th.). Entomologisches Archiv.

2 vols. $4^{\circ}$. Jena, 1827-29.

THON (Th.). Neue Schmetterlingsbelustigungen.

8․ Jena \& Leipzig, 1828.

THORELL (T.). Recensio critica Aranearum Suecicarum. $4^{\circ}$. Upsatice, 1856.

THORELL (T.). Bidrag till Kännedomen om Krustaceer. $4^{\circ}$. Stockholm, 1859.

THORELL (T.). On European Spiders.

Part I. 4\%. Upsala, 1869--70.

THORELL (T.). Remarks on Synonyms of European Spiders. roy. 8०. Upsala, 1871-73.

THORELL (T.). Verzeichniss sïdrussischer Spinnen.

80. St. Petersburg, 1875.

THORELL (T.). Descriptions of European and NorthAfrican Spiders.

$4^{\circ}$. Stockholm, 1875.

THORELL (T.). See 'Eugenie.'

THORLEY (J.). An Enquiry into the Nature, Order, and Government of Bees.

2nd edition. $8^{\circ}$. London, 1765. 
THORNER (E.). See Fisherres: Berin. Internationalo Fischerei-Ausstellung, 1880.

THORPE (C.). British Marine Conchology.

12. London, 1844.

THUET (M. J.). Disquisitiones anatomicæ Psittacorum.

$4^{\circ}$. Turici, 1838.

THUNBERG (C. P.). Dissertatio Entomologica, sistens Insecta Svecica. Parts 2, 3, 4, 5, 8, 9.

$4^{\circ}$. Upsal, 1791-95.

THUNBERG (C. P.). Beskrifning på Svenske Djur. Första Classen, om Mammalia eller Däggan de Djuren.

8. Upsala, 1798.

TIBERI (N.). See Benort (L.).

TIEDEMANN (F.). Zoologie zu seinen Vorlesungen entworfen. 3 vols. $8^{\circ}$. Landshut, 1808-14.

TIEDEMANN (F.). Anatomio des Fischherzens.

$4^{\circ}$. Landshut, 1809.

TIEDEMANN (F.). Anatomio und Naturgeschichte des Drachens. $4^{\circ}$. Nürnberg, 1811.

TIEDEMANN (F.). Anatomie der Röhren-Holothurio des Pomeranzfarbigen Seesterns und Stein-Seeigels.

Text and Atlas, fol. Landshut, 1816.

TIEDEMANN (F.). Icones Cerebri Simiarum et quorundam Mammalium rariorum. fol. Heidelbergce, 1821.

TIGNY (F. M. G. T. de). Histoire Naturelle des Insectes. 2nd edition. 10 vols. $16^{\circ}$. Paris, 1813.

TILESIUS (W. G.). Ueber die sogenannten Seemäuse, oder hornartigen Fischeyer, nebst anatomisch-physiologischen Bemerkungen über die Fortpflanzungsweise der Rochen und Hayfische.

$4^{\circ}$. Leipzig, 1802.

TODARO (F.). Contribuzione alla Anatomia e alla Fisiologia de tubi di senso de Plagiostomi. $4^{\circ}$. Messina, 1870. 
TODD (R. B.). The Cyclopædia of Anatomy and Physiology. $\quad 5$ vols. $8^{\circ}$. London, 1835-47. Supplement to same. $\quad 8^{\circ}$. London, 1859.

TOMES (C. S.). A Manual of Dental Anatomy.

8. London, 1882.

TOMLES (R. F.). See BeLl (T.).

TOPSEL (E.). The History of Four-footed Beasts and Serpents. fol. London, 1658.

TORELL (O.). Spitsbergens Molluskfauna.

8. Stockholm, 1859.

TOURNIER (H.). Description des Dascillides du Bassin du Léman.

8. Bale \& Genève, 1868.

TOWNSEND (J. K.). Narrative of a Journey across the Rocky Mountains.

8. Philadelphia, 1839.

TOWNSHEND (F. T.). Ten thousand miles of Travel, Sport, and Adventure.

$8^{\circ}$. London, 1869.

TOWNSON (R.). Observationes physiologicæ de Amphibiis. Pars prima de Respiratione. $4^{\circ}$. Gottingo, 1794.

TOZZETTI (A. T.). Relazioni sulla Pesca.

8. Genova, 1872.

TOZZETTI (A. T.). Notizie e Indicazioni sulla Malattia del Pidocchio della Vite o della Fillossera (Phylloxera vastatrix).

8.. Roma, 1879.

TOZZETTI (A. T.). See 'Magenta。’

TRAILL (T. S.). Description of a Silurus, known in Demarara by the name of Gilbacke, more properly Geelbuik.

8. Eclinburgh, 1826.

TRAIIL (T. S.). See Schlegel (H.).

TRAPANI (G.). Catalogue of the Fish of Malta and Gozo.

$8^{\circ}$. Malta, 1838. 
TREITSCHKE (F.). See Ochsenheimer (F.).

TREMRLEY (A.). Abhandlungen zur Geschichte einer Polypenart des süssen Wassers. Aus dem Französ. übers. von J. A. E. Goeze. 8. Quedlinburg, 1775.

TREUTLER (F. A.). Observationes pathologico-anatomicæ auctarium ad Helminthologiam Humani Corporis continentes. $4^{\circ}$. Lipsiae, 1793.

TRIMEN (R.). Rhopalocera Africæ Australis.

8. Cape Town, 1862-66.

TRISTRAM (H. B.). The Great Sahara.

8. . London, 1860.

TRISTRAM (H. B.). The Natural History of the Bible. 8. London, 1880.

TPITSCHE (H.). See SchrencK (L. v.).

TROIS (E. F.). See Fisheries : BerLin. Internationale Fischerei-Ausstellung, 1880.

TROSCHEL (F. H.). Symbolæ de Holodermate horrido. $4^{\circ}$. Bonno, 1851.

TROSCHEL (F. H.). Das Gebiss der Schnecken.

$4^{\circ}$. Berlin, 1856-63.

TROUESSART (E. L.). Revue Synoptique des Cheiroptères d'Europe. 8. Villenêque, 1879.

TRUQUI (E.). See Periodicals : 'Turin.

TRYON (G. W.). Structural and Systematic Conchology. 2 vols. $8^{\circ}$. Philadelphia, 1882-83.

TRYON (G. W.). Manual of Conchology.

4 vols. and parts. $8^{\circ}$. Philadelphia, 1879-83. [In progress.]

TRYON (G. W.). See Brnney (W. G.). 
TSCHUDI (A.). Die Blasenwürmer.

4․ Freiburg im Breisgau, 1837.

TSCHUDI (F. v.). Das Thierleben der Alpenwẹlt. Naturansichten und Thierzeichnungen aus dem Schweizerischen Gebirge.

8. Leipzig, 1872.

TSCHUDI (J. J.). Monographie der Schweizerischen Echsen. 4. Neuchâtel, 1837.

TSCHUDI (J. J.). Classification der Batrachier.

$4^{\circ}$. Neuchâtel, 1838.

TSCHUDI (J. J. von). Fauna Peruana.

fol. St. Gallen, 1844-46.

TSCHUSI-SCHMIDHOFEN ( $V$. R. von). Der Tannenheher (Nucifraga caryocatactes). $\quad 4^{\circ} . \quad$ Dresden, 1873.

TSCHUSI-SCHMIDHOFEN ( $\nabla . \quad R$. von). Die Vögel Salzburg's. 8. Salzburg, 1877.

TUANO (J. A.). Il Falconiere : coll' Uccellatura di P. A. BARGEO. $4^{\circ}$. Venezia, 1735.

TUCH (J. A.). Descriptio osteologica capitis Myrmecophagce jubatce. $4^{\circ}$. Berolini, 1821.

TUGWELL (G.). A Manual of Sea-Anemones.

8. London, 1856.

TULK (A.) \& HENFREY (A.). Anatomical Manipulation.

12. London, 1844.

TUNSTALL (M.). Ornithologia Britannica.

fol. London, 1771.

TUNSTALL(M.). See Periodicals : London. Willughby Society.

TURNBULL (W. P.). The Birds of East Pennsylvania and New Jersey.

8․ Glasgow, 1869.

TURNER (W.). See 'Challenger.' 
TURTON (W.). British Fauna. $\quad 12^{\circ}$. Swansea, 1807.

TURTON (W.). Conchylia Insularum Britannicarum. $4^{\circ}$ Exeter, 1822.

TURTON (W.). Manual of the Land and Freshwater Shells of the British Islands, revised by J. E. GraY.

$8^{\circ}$. London, 1840.

TURTON (W.). Manual of the Land and Freshwater Shells of the British Islands, with additions by J. E. GRAY. 8. London, 1857.

TWEEDDALE (Arthur, Marquis of). Ornithological Works. Edited by R. G. Wardlaw Ramsay. Biographical sketch by W. H. RusselL.

$4^{\circ}$. London, 1881.

TWEEDDALE (Arthur, Marquis of). See ' Challenger.'

TYLOR (E. B.). Anthropology : an Introduction to the Study of Man and Civilization. 8. London, 1881.

TYSON (E.). The Anatomy of a Pygmie.

$4^{\circ}$. London, 1699.

U.

UDMAN (J.). Noræ Insectorum Species. Editio altera curante G. W. F. PANzer. $4^{\circ}$. Erlange, 1793.

UHLER (P. R.). See Reports : Washington, U.S.

UHLER (P. R.). See Survers \& Explorations : U.S., WASHINGTON.

ULKE (H.). See Reports: Washington, U.S.

ULKE (H.). See Survers \& Explorations : U. S., WASHINGTON.

ULLERICH (A.). Der Japanische Eichenspinner (Bombyx yаma-mayou).

8. Eichstcett, 1870. 
ULMANN (L.). Disquisitiones.

8. Dorpati Livonorum, 1855.

ULRICH (A. L.). Annotationes de Sensu ac Significatione ossium capitis speciatim de capite Testudinis.

$4^{\circ}$. Berolini, 1816.

' URANIE' \& 'PHYSICIENNE.' Voyage autour du Monde, pendant les années 1817, 1818, 1819 et 1820, par L. DE Fretcinet.

Zoologie par MM. Qtor et GaImaRd.

Texte, $4^{\circ}$. Planches, fol. Paris, 1824.

$\nabla$.

VAILIANTE (R.).' See FatNa UNd Flora des Golfes von Neapel.

VAILLANT (F. le). Travels from the Cape of Good Hope into the Interior Parts of Africa.

2 vols. $8^{\circ}$. London, 1791.

Another edition. 3 vols. $8^{\circ}$. London, 1796.

See Levalulant.

VAillant (L.). See Strveys \& Explorations: France.

VALENCIENNES (A.). Ichthyologie des Iles Canaries.

$4^{\circ} . \quad$ Paris, 1838.

VALENCIENNES (A.). See Belanger (C.).

VALENCIENNES (A.). See CUtier (G.).

VALENTINI (M. B.). Amphitheatrum Zootomicum.

fol. Francofurti, 1720.

VALLARDI (F.). See Fauna d' Italia.

VALLISNIERI (A.). Considerazioni, ed Esperienze intorno al creduto Cervello di Bue impietrito, vivente ancor l' animale.

$4^{\circ} . \quad$ Padoa, 1710. 
VALLISNIERI (A.). Opere Diverse.

1. Istoria del Camaleonte Africano, e di varj Animali d' Italia.

2. Lezione Accademica intorno all' Origine delle Fontane.

4. Raccolta di varj Trattati accresciuti con Annotazioni, e con Giunte. $\quad 4^{\circ} . \quad V$ enezia, 1715.

VALLOT (J. N.). Concordance Systématique, servant de table de mattières à l'ourrage de Réaumur, intitulé: Mémoires pour servir à l'Histoire des Insectes.

$4^{\circ}$. Paris, 1802.

VALLOT (J. N.). Ichthyologie Française ou Histoire Naturelle des Poissons d'Eau douce de la France. Arec Supplément. 8. Dijon, 1837.

VANDELLI (D.). Dissertationes Tres.

8. Patavii, 1758.

VAN DER HOEVEN (J.). See Hoeven (J. van der).

VAN DER HOEveN (J.). See Periodicals : The Hague.

VASEY (G.). Delineations of the Ox Tribe.

8. London, 1851.

VEJDVOSKÝ (F.). Beiträge zur vergleichenden Morphologie der Anneliden. I. Enchytraeiden.

$4^{\circ}$. Paris, 1879.

VEJDOVSKÝ (F.). Untersuchungen über die Anatomie, Physiologie und Entwicklung von Sternaspis.

$4^{\circ}$. Wien, 1881.

VEJDOVSKÝ (F.). Thierische Organismen der Brunnenwässer von Prag.

$4^{\circ}$. Prag, 1882.

VEJDOVSKÝ (F.). Revisio Faunæ Bohemicæ. Pars I. Die Süsswasserschwämme Böhmens. $\quad 4^{\circ}$. Prag, 1883.

VENNOR (H. G.). Our Birds of Prey of Canada. $4^{\circ}$. Montreal, 1876. 
'VENUS.' Voyage autour du Monde sur la Fregatte 'Venus,' commandée par A. Du Petit-Thouars.

Zoologie. Atlas, fol. Text, $8^{\circ}$. Paris, 1846-55. Mammifères, par M. J. Geoffroy Saint-Hilaire. Oiseaux, par F. Prevost \& O. Des Murs. Reptiles, par M. Domeric.

VERANY (G. B.). Catalogo degli Animali Invertebrati Marini del Golfo di Genova e Nizza. $\quad 8^{\circ}$. Genova, 1846.

VERANY (J. B.). Mollusques Méditerranéens. Part 1. Cephalopodes. $4^{\circ}$. Gènes, 1851.

VERARDI (M.). Manuel du Destructeur des Animaux nuisibles.

$12^{\circ}$. Paris, 1827.

VERLOREN (M. C.). Commentatio de organis generationis in Molluscis. $\quad 4^{\circ}$. Lugduni Batavorum, 1837.

VERLOREN (II. C.). See Periodicals : The Hague.

VERRILL (A. E.). See Reports: Washington, U.S.

VERRILL (A. E.). See Survers \& Explorattons: U. S., WaSHINGTON.

VICTORIN (J. F.). Zoologiska Anteckningar under en resa i södra delarno af Caplandet, 1853-55.

$4^{\circ}$. Stockholm, 1858.

VIEILLOT (L. P.). Histoire Naturelle des Oiseaux de l'Amérique septentrionale. 2 vols. fol. Paris, 1807.

VIEILLOT (L. P.). Analyse du système général d'Ornithologie.

$8^{\circ}$. Paris, 1816.

VIEILLOT (L. P.). C. J. Temminck, Observations sur la Classification méthodique des Oiseaux.

8.. Amsterdam, 1817.

VIEILLOT (L. P.). See Faune Française.

VIEILLOT (L. P.) \& OUDART (P.). La Galerio des Oiseaux.

2 vols. $4^{\circ}$. Paris, 1825. 
VIEWEG (C. F.). Tabellarisches Verzeichniss der in der Churmark Brandenburg einheimischen Schmetterlinge.

$4^{\circ}$. Berlin, 1789-90.

VIEYRd. See Dictionaries. Portuguese-English \&c.

VIGORS (N. A.). See 'Blossom.'

VILLA (A.). Catalogo dei Molluschi della Lombardia.

8. Milano, 1844.

VILLA (A.). Straordinaria apparizione di Insetti carniFori.

$4^{\circ}$. Milano, 1860.

VILLA (A.). Sull' origine delle Perle.

8. Milano, 1860.

VILLERS (C. J. de). See Linveus (C.).

VINCENT (L.). Descriptio Pipæ, seu Bufonis aquatici Surinamensis .... addita succincta descriptione ranarum. [Description du Pipa.]

$4^{\circ}$. La Haye, 1726.

VINCENT (L.). Catalogus et descriptio Animalium Volatilium, Reptilium, et Aquatilium. [Title also in French.]

$4^{\circ}$. La Haye, 1726.

VINSON (A.). Aranéides des Iles de la Réunion, Maurice et Madagascar. $\quad$ 80. Paris, 1863.

VINSON (A.). Voyage à Madagascar. $\quad 8^{\circ}$. Paris, 1865 .

VINTSCHGAU (M. v.). See Hermand (L.). Handbuch der Physiologie.

VIREY (J. J.). Histoire des Mœurs et de l'Instinct des Animaux.

2 vols. 8o. Paris, 1822.

VOET (J. E.). Catalogus Systematicus Coleopterorum.

$4^{\circ}$. La Haye, 1806.

VOGEL (C. F.). Chronologischer Raupenkalender oder Naturgeschichte aller Europäischen Raupen.

3rd edition. $8^{\circ}$. Berlin, 1845. 
VOGT (C.). Zur Anatomie der Amphibien.

$4^{\circ}$. Bern, 1839.

VOGT (C.). Untersuchungen über die Entwicklungsgeschichte der Geburtshelferkrœte (Alytes obstetricans).

$4^{\circ}$. Solothurn, 1842.

VOGT (C.). Sur les Siphonophores de la Mer de Nice.

$4^{\circ}$. Genève, 1854.

VOGT (C.). Die Künstliche Fischzucht.

8. Leipzig, 1875.

VOGT (C.). Die Herkunft der Eingeweidewürmer des Menschen.

8. . Basel, 1878.

VOIT (C. v.). See Hermann (L.). Handbuch der Physiologie.

VOLBORTH (A.). De Bobus Uro, Arni et Caffro.

$4^{\circ}$. Berolini, 1825.

VOLBORTH (A. von). Ueber die mit glatten Rumpfgliedern versehenen Russischen Trilobiten.

$4^{\circ}$. St. Petersburg, 1863.

VOLBORTH (A. von). Ueber einige neue Ehstländische Illaenen.

$4^{\circ}$. St. Petersburg, 1864.

VOLBORTH (A. von). Ueber Achradocystites und Cystoblastus, zwei neue Crinoideen-Gattungen.

$4^{\circ}$. St. Petersburg, 1870.

VOLKMANN (A. G.). Anatomia animalium.

$4^{\circ}$. Lipsiae, 1831.

VOLLENHOVEN (S. C. S.). See S Svellen vaN VollenHOVEN (S. C.).

VON DER DECKEN (C. C.). Reisen in Ost-Afrika.

6 vols. $8^{\circ}$. Leipzig, 1869.

VOSMAER (A.). Beschryving van Africaansch, Oostindischen, Amerikaanschen \&c. zeldzame gedierten.

$4^{\circ}$. Amsterdam, 1766-87. 
VOSMAER (A.). Regnum Animale. Description d'un Recueil exquis d'Animaux rares. 40. Amsterdam, 1804.

VOSMAER (G. C. J.). See Brorn (H. G.).

VOSIIAER (A.). See RENaRd (L.).

VOYAGES. See 'Alert,' 'Betgle,' 'Belayger,' 'Blossour,' 'Bontre,' 'Bulldog,' 'Challenger,' 'Curaçoa,' 'Corwin,' 'Erebus' atd 'Terror,' 'Evgenie,' ' Fafortte,' ' Herald,' 'Magenta,' 'Notara,' 'Samaraig,' 'Uranie' \& 'PhySICIENNE,' 'VENCs.'

VROLIK (W.). Specimen anatomico-zoologicum de Phocis. 8. Trajecti ad Rhenum, 1822.

VROLIK (W.). Natuur- en ontleedkundige opmerkingen over den Chameleon.

$8^{\circ}$. Amsterdam, 1827.

VROLIK (W.). Recherches d'anatomie comparée sur le Chimpansé.

fol. Amsterdam, 1841.

W.

WACHTL (F. A.). Die Weisstannen-Triebwickler, Tortrix muriana.

$4^{\circ}$. Wien, 1882.

WAFER (L.). See DaMpier (W.).

WAGLER (J.). Systema Avium. 120. Stuttgart, 1827.

WAGLER (J.). Descriptiones et Icones amphibiorum.

fol. Monachi \& Stuttg., 1833.

WAGLER (J.). Monographia Psittacorum.

4. München, 1835.

WAGLER (J.). See Spix (J. B. de).

WAGNER (A.). De Spatulariarum Anatome.

4․ Berolini, 1848. 
WAGNER (J.). De partibus Mammalium os temporum constituentibus. 8. Dorpati Livon., 1858.

WAGNER (J. A.). See SPIx (J. B.).

WAGNER (M.). Reisen in der Regentschaft Algier in den Jahren 1836, 1837 und 1838.

3 vols. Text, $8^{\circ}$. Atlas, $4^{\circ}$. Leipzig, 1841.

WAGNER (R.). Beiträge zur Anatomie der Vögel. 4. München, 1832.

WAGNER (R.). Prodromus Historiæ Generationis Hominis atque Animalium. fol. Lipsia, 1836.

WAGNER (R.). Icones Zootomicæ. Handatlas zur vergleichenden Anatomie. $\quad 4^{\circ}$. Leipzig, 1841.

WAGNER (R.). Ueber den Bau der Pelagia noctiluca und die Organisation der Medusen, zugleich als Prodromus seines zootomischen Handatlasses. fol. Leipzig, 1841.

WAGNER (R.). Lehrbuch der Anatomie der Wirbelthiere. 8.. Leipzig, 1843.

WAHLBERG (J. A.). Insecta Caffraria. See BoHeman (C. H.). 2 vols. $8^{\circ}$. Holmice, 1848.

WAHLSTRÖM (J. E.). Svensk exkursions-Fauna. 1. Ryggradsdjuren. 12. Stockholm, 1867.

WAHNSCHAFFE (M.). Verzeichniss der im Gebiete des Aller-Vereins zwischen Helmstedt und Magdeburg aufgefundenen Käfer.

$8^{\circ}$. Neuhaldensleben, 1883.

WALBAUM (J. J.). See ARtedi (P.).

WALBAUM (J. J.). See KleIN (J. T.). Ichthyologia Enodata.

WALCKENÆR (C. A.). Faune Parisienne (Insectes) ou Histoire Abrégée des Insectes des environs de Paris.

2 vols. $8^{\circ}$. Paris, 1802. 
WALCKENAER (C. A. de). Histoire naturelle des Insectes (Aptères). $\quad 4$ vols. $8^{\circ}$. Puris, 1837-47.

WalckenaER (C. A.). See Fadne Franģatse.

WALECKIEGO (A.). Materyaly do Fauny Ichtyologicznej Polski. 8. Warsaw, 1864.

WALKER (F.). Monographia Chalciditum. 2 vols. $8^{\circ} . \quad$ London, 1839.

WALKER (F.). Insecta Saundersiana. Diptera.

2 vols. $8^{\circ}$. London, 1850.

WALKER (F.). Ins ecta Britannica. Diptera.

3 vols. $8^{\circ}$. London, 1851-56.

WALKER (F.). Catalogue of Heteropterous Hemiptera in the British Museum. Vols. I.-VIII.

$8^{\circ}$. London, 1867-73.

WALKER (F.). Characters of undescribed Lepidoptera Heterocera.

8. Lonclon, 1869.

WALKER (F.). List of Coleoptera collected by J. K. Lord in Egypt and Arabia. 8. London, 1871.

WALKER (F.). A list of Hymenoptera collected by J. K. Lord in Egypt.

8.. London, 1871.

WALKER (F.). Notes on Chalcididce. Parts I. to VII. $8^{\circ}$. London, 1872.

WALKER (F.). Notes on Diptera and lists of Species. 1st series. $8^{\circ}$. London, 1874.

WALKER (F.). Translation of Synoptical arrangements of some European Hymenoptera.

8. . London, 1874.

WALKER (F.). See MUsedm (British).

WALKER (F.). See Sadnders (W. W.).

WALKER (F. A.). L'Orient: or, a Journal of my Tour in the East. $8^{\circ}$. London, 1882. 
WALL (W. S.). On the Skeleton of a new Sperm Whale lately set up in the Australian Museum. $8^{\circ}$. Sydney, 1851.

WALLACE (A. R.). The Malay Archipelago.

2 vols. $8^{\circ}$. London, 1869.

WALLACE (A. R.). The Geographical Distribution of Animals. 2 vols. $8^{\circ}$. London, 1876.

WALLEM (F. M.). See FisherIes: London. International Fisheries Exhibition, 1883.

WALLENGREN (H. D. J.). Skandinaviens Dagfjärilar.

8. Malmö, 1853.

WALLENGREN (H. D. J.). Skandinaviens Heterocer Fjärilar. 3 Häft. $8^{\circ}$. Lund, 1863, 1869, 1871.

WALLENGREN (H. D. J.). See 'Eugenie.'

WALLICH (G. C.). Deep-sea Researches on the Biology of Globigerina.

$8^{\circ}$. London, 1876.

WALLICH (G. C.). See 'BULldoG.'

WALLON (E.). Questions de Pêche et d'Aquiculture fluviales et maritimes. $\quad 8^{\circ}$. Montauban, 1868.

WALPOLE (Spencer). See FisherIes : London.

WALSH (R.F.). See Fisheries : London. International Fisheries Exhibition, 1883.

WALSINGHAM (Thomas De Grey, Lord). Illustrations of typical specimens of Lepidoptera Heterocera in the British Museum. Part IV. North-American Tortricidæ.

See Museum (British).

$4^{\circ}$. London, 1879.

WALSINGHAM (Thomas De Grey, Lord). Pterophoridae of California and Oregon.

8. London, 1880.

WALTL (J.). Reise durch Tyrol. $\quad 8^{\circ} . \quad$ Passau, 1835.

WALTON (Elijah). The Camel : its Anatomy, Proportions, and Paces.

fol. London, 1865. 
WALTON (J.). On the British Curculionidae.

8. London, 1844.

WALTON (J.). See Musedm (Britist).

WARDER (J.). The true Amazons : or, the Monarchy of Bees.

$12^{\circ}$. London, 1749.

WATERHOUSE (C. O.). Aid to the Identification of Insects. Vol. I. and Parts. sm. 40. London, 1880-83. [In progress.]

WATERHOUSE (C. O.). See Musedm (British).

WATERHOUSE (G. R.). Catalogue of the Mammalia in the Museum of the Zoological Society.

2nd edition. $8^{\circ}$. London, 1838.

WATERHOUSE (G. R.). A Natural History of the Mammalia. 2 vols. $8^{\circ}$. London, 1846-48.

WATERHOUSE (G. R.). Catalogue of British Coleoptera. $8^{\circ}$. London, 1858.

WATERHOUSE (G. R.). Pocket Catalogue of British Coleoptera. $12^{\circ}$. London, 1861.

WATERHOUSE (G. R.). See 'Beagle.'

WATERTON (C.). Essays on Natural History. 1st series. 5th edition. $12^{\circ}$. London, 1844. 2nd series. 2nd edition. $12^{\circ}$. London, 1844.

WATSON (F.). The Animal World Displayed.

8.. London, 1754.

WATSON (M.). See 'Challenger.'

WEBB \& BERTHELOT. See Museum (Britisn).

WEBER (E. H.). De Aure et Auditu Hominis et Animalium. $4^{\circ}$. Lipsice, 1820.

WEBER (F.). Nomenclator Entomologicus secundum Entomologiam systematicam ill. Fabricii.

12. Chilonii \& Hamburgi, 1795. 
WEBER (F.). Observationes Entomologicæ.

$12^{\circ}$. Kilice, 1801

WEBER (J. C.). Die Fische Deutschlands -und der Schweiz. $12^{\circ}$. München, 1870.

WEDDERBURN (J. W.). See JoNes (J. M.).

WEIDEMEYER (J. W.). Catalogue of North American Butterflies.

8․ Philadelphia, 1864.

WEILER (J.). Verzeichniss der Schmetterlinge von Innsbruck und dessen Umgebung, mit Berücksichtigung der nordtirolischen Lepidopteren überhaupt.

$8^{\circ}$. Innsbruck, 1877.

WEILER (J.). Die Schmetterlinge des Tauferer Thales. Ein Beitrag zur Lepidopteren-Kunde von Tirol.

$8^{\circ}$. Innsbruck, 1880.

WEINKAUFF (H. C.). Catalog der im Europäischen Faunengebiet lebenden Meeres-Conchylien.

$8^{\circ}$. Kreuznach, 1873.

WEINKAUFF (H. C.). Die Conchylien des Mittelmeeres, ihre geographische und geologische Verbreitung.

2 vols. $8^{\circ}$. Cassel, 1867-68.

WEINLAND (D. F.). An Essay on the Tapeworms of Man. 8.. Cambridge, Mass., 1858.

WEINLAND (D. F.). Führer durch den Zoologischen Garten in Frankfurt am Main. $\quad 12^{\circ}$. Frankfurt, 1865.

Weinland (D. F.). See Periodicals : Frankfurta. M.

WEIR (H.). See Tegetmeier (W. B.).

WEISMANN (A.). Die Entwicklung der Dipteren.

8. Leipzig, 1864.

WEISMANN (A.). Ueber die Berechtigung der Darwin'schen 'Theorie.

8. Leipzig, 1868. 
WEISMANN (A.). Ueber den Einfluss der Isolirung auf die Artbildung. 8. Leipzig, 1872.

WEISMANN (A.). Studien zur Descendenz-Theorie.

Parts I. \& II. $8^{\circ}$. Leipzig, 1875-76.

WEISMANN (A.). Das Thierleben im Bodensee.

$$
\text { 8. Lindau, } 1877 .
$$

WEISMANN (A.). Studies in the Theory of Descent. Translated by R. Meldola. With Prefatory Notice by C. DARIIIN. 2 vols. $8^{\circ}$. London, 1882.

WEISMANN (A.). Die Entstehung der Sexualzellen bei den Hydromedusen. $\quad 4^{\circ}$ Text and Atlas. Jena, 1883.

WEISSE (J. F.). Zur Oologie der Räderthiere.

4\%. St. Petersburg, 1862.

WELLENBERGH (P. H. J.). Observationes anatomicie de Orthagorisco mola. $\quad 4^{\circ}$. Lugduni Batavorum, 1840.

WELLSTED (J. R.). Travels in Arabia.

2 vols. $8^{\circ}$. London, 1838.

WELWITSCH (F.). Voyage dans les royaumes d'Angola et de Benguella. Mollusques terrestres et fluviatiles par Arthor Morelet.

$4^{\circ}$. Paris, 1868.

WENCKER (J.) \& SILBERMANN (G.). Catalogue des Coléoptères de l'Alsace et des Vosges.

Espèces nouvelles par CH. Brisout de Barneville et WENCKER. 80. Strasbourg, 1866.

WERNEBURG (A.). Beiträge zur Schmetterlingskunde. 2 vols. $8^{\circ}$. Erfurt, 1864.

WESSEL (A. W.). Beitrag zur Käferfauna Ostfrieslands. $8^{\circ}$. Aurich, 1877.

WESTERLUND (C. A.). Fauna Molluscorum Sueciæ, Norvegix et Daniæ. 2 vols. $\quad 8^{\circ}$. Stockholm, 1873.

WESTERLUND (C. A.). Fauna Europæa : Molluscorum extramarinorum Prodromus. $\quad 8^{\circ}$. Lunde, 1876-78. 
WESTERLUND (C. A.). Monografi öfver Paläoartiska Regionens Clausiler. $\quad 8^{\circ}$. Lund, 1878.

WeSterdyan (G. F.). See Periodicals: Austerday.

WESTWOOD (J. O.). The Entomologist's Text Book.

$12^{\circ}$. London, 1838.

WESTWOOD (J. O.). Introduction to the Modern Classification of Insects. 2 vols. 8. London, 1839-40.

WESTWOOD (J. O.). Arcana Entomologica.

2 vols. $8^{\circ}$. London, 1845.

WESTWOOD (J. O.). Cabinet of Oriental Entomo$\log y$. $4^{\circ}$. London, 1848.

WESTWOOD (J. O.). The Butterflies of Great Britain, with their Transformations.

8. London, 1855.

WESTWOOD (J. O.). Catalogue of Orthopterous Insects. Part I. Phasmidce. 4\%. London, 1859. See Museum (British).

WESTWOOD (J. O.). Thesaurus Entomologicus Oxoniensis. $4^{\circ}$. Oxford, 1874.

WESTWOOD (J. O.). See Donovan (E.).

WESTWOOD (J. O.). See DrdRY (D.).

WESTWOOD (J. O.). See HuMphreys (H. N.).

WESTWOOD (T.) \& SATCHELL (T.). Bibliotheca Piscatoria. 8. London, 1883.

WETTER (J. J.). Erinacei Europæi anatome. $8^{\circ}$. Gottingce, 1818.

WEYENBERG(H.). Hypostomus plecostomus : Mémoire anatomique. 8. Cordoba \& Leipzig, 1876.

WEYENBERG (H.). Algunos nuevos Pescados del Museo Nacional y alguras noticias ictiológicas.

$4^{\circ}$. Buenos Aires, 1877. 
WHALE. History of the great Whale captured near Havre, and landed at Langston Harbour, 20th November, 1869.

$12^{\circ}$. Portsmouth, 1873 .

WHEELDON (J. P.). See Fisheries : London. International Fisheries Exhibition, 1883.

WHEELER (G. M.). See Reports : Washington, U. S.

WHEELER (G. M.). See Surveys \& Explorations : WASHINGTON, U.S.

WHEELWRIGHT (H. W.). Comparative List of Birds of Scandinavia and Britain. $\quad 4^{\circ}$. Carlstad, 1852-59.

WHEELWRIGHT (H. W.). See Bushman, Ord (pseud.).

WHITE (A.). A Popular History of British Crustacea. sm. $4^{\circ}$. London, 1857.

WHITE (A.). See 'Erebus' \& 'Terror.'

WhiTE (A.). See 'Samarang.'

WHITE (B.). See 'Challenger.'

WHITE (G.). A Naturalist's Calendar, with observations in various branches of Natural History.

8.. London, 1795.

WHITE (G.). The Natural History and Antiquities of Selborne. $4^{\circ}$. London, 1813.

WHITE (G.). The Natural History and Antiquities of Selborne. Edited by J. E. Harting. 8 ${ }^{\circ}$. London, 1876.

WHYMPER (F.). Travels and Adventures in the Territory of Alaska. 2nd edition. $8^{\circ}$. London, 1869.

WIEDEMANN (C. R. W.). Diptera Exotica. 8․ Kilice, 1821.

WiedemanN (C. R. W.). See Periodicals : Berlin. 
WIEDEMANN (C. R. W.). Achias, Dipterorum Genus; illustratum novisque speciëbus anctum.

$8^{\circ}$. Kilice Holsutorum, 1830.

Wiedemann (C.R. W.). See Periodicals : Berlin and KIEL.

WIEDERSHEIM (R.). Dio Anatomio der Gymnophionen. $4^{\circ}$. Jena, 1879 .

WIEDERSHEIM (R.). Lehrbuch der vergleichenden Anatomie der Wirbelthiere. $8^{\circ}$. Jena, 1883.

WIED-NEUWIED (Maximilian, Prinz von). Reise nach Brasilien in den Jahren 1815-17.

2 vols. $4^{\circ}$. Atlas, fol. Frankfurt, 1820-21.

WIED-NEUWIED (Maximilian, Prinz von). Abbildungen zur Naturgeschichte Brasiliens. fol. Weimar, 1822-31.

WIED-NEUWIED (Maximilian, Prinz von). Beiträge zur Naturgeschichte von Brasilien. $8^{\circ}$. Weimar, 1825-33.

WIED-NEUWIED (Maximilian, Prinz von). Brasilien. Nachträge Berichtigungen und Zusätze zu der Beschreibung meinerReise im östlichen Brasilien.

8. Frankfurt a. Main, 1850.

WIED-NEUWIED (Maximilian, Prinz von). Verzeichniss der auf seiner Reise in Nord-America beobachteten Säugethiere.

8. Berlin, 1862.

WIEG】ANN (A. F. A.). Observationes zoologicæ criticse in Aristotelis Historiam Animalium. $\quad 4^{\circ}$. Lipsia, 1826.

WIEGMANN (A. F. A.) \& RUTHE (J. F.). Handbuch der Zoologie.

$8^{\circ}$. Berlin, 1832.

Wieguann (A. F. A.). See Pertodicals : Berlin.

WILCOCKS (J. C.). The Sea-Fisherman, or Fishing Pilotage. $8^{\circ}$. Guernsey, 1865.

WILDE (O.). Die Pflanzen und Raupen Deutschlands.

8. Berlin, 1860-61. 
WILKEN (C.). Käfer-Fauna Hildesheims.

$12^{\circ}$. Hildesheim, 1867.

WILKES (B.). One hundred and twenty copper plates of English Moths and Butterflies. $\quad 4^{\circ}$. London, 1773.

WILKES (C.). See Explorations \& Surteys: U. S., Philadelphia.

WILKINSON (S. J.). The British Tortrices.

8. London, 1859.

WILLEMIOES-SUHM ( $\mathrm{R}$. von). Ueber einige Trematoden und Nemathelminthon.

8. Leipzig, 1870.

WILLEMOES-SUHM (R. von). Challenger-Briefe von R. $\nabla$. Willenoes-SuHm, 1872-1875. 80. Leipzig, 1877.

WILLIAMISON (W. C.). Recent Foraminifera of Great Britain.

See Ray Societr.

fol. London, 1858.

WILLUGHBY(F.). Ichthyographia. $4^{\circ}$. Londini, 1685.

WILLUGHBY (F.). Ornithologiae libri tres in quibus Ares omnes hactenus cognitae \&c. fol. Londini, 1676.

WILLUGHBY (F.). The Ornithology of F. W. By JoнN RAY, F.R.S.

fol. London, 1678.

WILLUGHBY (F.). De Historia Piscium Libri Quatuor. Totum Opus recognovit, Librum etiam primum et secundum integros adjecit $J$. RaIUS.

$4^{\circ}$. Oxonii, 1686.

WIIS (H. B.). Dissertatio medica.

8. Lugduni Batavorum, 1844.

WIISON (A.). American Ornithology.

1st edition. 9 vols. $4^{\circ}$. Philadelphia, 1808-14.

WILSON (A.). The Student's Guide to Zoology.

8․ London, 1874. 
WILSON (A.), BONAPARTE (C. L.), \& JARDINE (W.). American Ornithology.

3 vols. $8^{\circ}$. London \& Edinburgh, 1832.

WILSON (A.) \& BONAPARTE (C. L.). American Ornitholngy; or, The Natural History of the Birds of the United States. [Ed. S. F. BAIRd.]

Text, 3 vols. $8^{\circ}$. Plates, 2 vols. fol. Philadelphia, n. d.

WILSON (J.). Illustrations of Zoology.

fol. Edinburgh \& London, 1831.

WILSON (O. S. \& E). The Larræ of the British Lepidoptera and their Food-Plants.

8. London, 1880.

WINCKELL (G. F. D.). Handbuch für Jäger. Herausgegeben von TscHUd.

2 vols. $8^{\circ}$. Leipzig, 1878.

WIXKER (F. A.). Observationes anatomicae de Tatu novemcincto. $4^{\circ}$. Tubinga, 1826.

WINKLER (T. C.). Des Tortues fossiles. roy. $8^{\circ}$. Harlem, 1869.

WiTTICH (W. v.). See Hermann (L.). Handbuch der Physiologie.

WitTyaCK (L.). See Fisheries: Berlin.

WODARCH (C.). Introduction to the study of Conchology. Edited by J. Mawe. $\quad 8^{\circ}$. London, 1827.

WOLF (J.). Abbildungen und Besschreibungen merkwürdiger naturgeschichtlicher Gegenstände.

2 vols. $4^{\circ}$. Nü̈rnberg, 1816-1822.

TOLF (J.). Zoological Sketches. Edited by P. L. Sclater. fol. London, 1861.

WOLFF (J.). Untersuchungen über die Entwicklung des Knochengewebes.

8. Leipzig, 1875.

WOLFF (J. F.). Abbildungen der Wanzen.

$4^{\circ}$. Erlangen, 1800. 
WOLFF (L.). Dissertatio Anatomica de Organo Vocis Mammalium. $\quad 4^{\circ} . \quad B e r l i n i, 1812$.

WOLLASTON (T. V.). Catalogue of the Madeiran Insects in the British Museum.

$4^{\circ} . M S$.

WOLLASTON (T. V.). Coleoptera Maderensia.

$4^{\circ}$. London, 1854.

WOLLASTON (T. V.). Insecta Maderensia.

$4^{\circ}$. London, 1854.

WOLLASTON (T. V.). On the Variation of Species, with especial reference to the Insecta.

8. London, 1856.

WOLLASTON (T. V.). Coleoptera Atlantidum.

8. London, 1865.

WOLLASTON (T. V.). Coleoptera Hesperidum.

8. . London, 1867.

WOLLASTON (T. V.). Coleoptera Sanctæ-Helenæ.

8. London, 1877.

WOLLASTON (T. V.). Testacea Atlantica.

8. London, 1878.

WOLLaSton (T. V.). See Musedm (British).

WOLLEY (J.). Ootheca Wolleyana. Edited by ALFRed Newton. Part I. Accipitres. roy. $8^{\circ}$. London, 1864.

WOOD (Capt.). See Dampier (W.).

WOOD (C. T.). The Ornithological Guide.

8․ London, 1835.

WOOD (J. G.). My Feathered Friends.

8. London, 1858.

WOOD (J. G.). Homes without Hands.

8. London, 1868 
WOOD (J. G.). The Natural History of Man.

$8^{\circ}$. London, 1868.

WOOD (J. G.). The Illustrated Natural History.

4 vols. $8^{\circ}$. London, n. d.

WOOD (J. G.). Insects at Home. $\quad 8^{\circ} . \quad$ London, 1872.

WOOD (J. G.). Bible Animals. $\quad$ 8. London, 1876.

WOOD (J. G.). Insects Abroad. $\quad$ 8․ London, 1883.

WOOD (N.). The Ornithologist's Text-Book.

8. London, 1836.

WOOD(W.). Zoography; or the Beauties of Nature displayed. 3 vols. $8^{\circ}$. London, 1807.

WOOD (W.). General Conchology. $\quad 8^{\circ}$. London, 1815.

WOOD (W.). Illustrations of the Iinnæan Genera of Insects. 2 vols. in $1.12^{\circ}$. London, 1821.

WOOD (W.). Catalogue of an extensive and valuable Collection of the Best Works on Natural History.

8.. London, 1824.

WOOD (W.). Index Testaceologicus. $8^{\circ}$. London, 1825. Supplement. $\quad 8^{\circ}$. London, 1828.

WOOD (W.). Index Entomologicus. 80. London, 1839.

WOOD (W.) \& HANLEY (S.). Index Testaceologicus.

$8^{\circ}$. London, 1856.

WOODS (W. Fell). Letters on Oyster Fisheries.

8. London, 1877.

WOODWARD (S. P.). A Manual of the Mollusca. post $8^{\circ}$. London, 1851. 3rd edition. post $8^{\circ}$. London, 1875.

WOODWARD (S. P.). Generic Catalogue of Recent and Fossil Shells.

$4^{\circ}$. Weymouth, 1859. 
WOTTON (E.). De differentiis Animalium.

fol. Lutetice Parisiorum, 1552.

WRAXALL (L.). Life in the Sea. $\quad 8^{\circ}$. London, 1860.

WRIGHT (E. P.). Six Months at the Seychelles.

$8^{\circ}$. Privately printed, 1868.

WRIGHT (E. P.). Spicilegia Biologica.

8. Privately printed, 1868-70.

WRIGHT (W.). See Fries (B. F.).

WULFEN (X.). Descriptiones quorundam Capensium

Insectorum.

$4^{\circ}$. Erlangae, 1786.

WULFF (J. C.). Ichthyologia cum Amphibiis regni Borussici. 8. Regiomonti, 1765.

WUNSCHE (O.). See Schlechtendal (D. H. R.).

WYMAN (J.). Anatomy of the nervous system of Rana pipiens. $4^{\circ}$. 1852 .

Y.

YARRELL (W.). On the growth of the Salmon in Fresh Water. obl. fol. London, 1839.

YARRELL (W.). A History of British Fishes. 2nd edition. 2 vols. $8^{\circ}$. London, 1841. 3rd edition by Sir J. RICHARDSON.

2 vols. $8^{\circ}$. London, 1859.

YARRELL (W.). A History of British Birds. 2nd edition.

3 vols. $8^{\circ}$. London, 1845. 4th edition by A. Newton \& H. Saunders.

Vols. I., II., and Parts. $8^{\circ}$. London, 1871-83. [In progress.] 
YARROW (H. C.). See Reports: Washington, U.S.

YARROW (H. C.). See Surters \& Explorations: U.S., WASHINGTON.

YAUVILLE (- d'). Traité de Ténerie.

$4^{\circ}$. Paris, 1788.

YONGE (C. D.). See Dictionaries, English-Greek.

YOUATT (W.). The Dog.

8. London, 1854.

YOUATT (W.). The Horse.

8. London, 1855.

YOUNG (A.). See HoLdsworti (E. W. H.).

Z.

ZADDACH (E. G.). De Apodis Cancriformis Schaeff. anatome et historia evolutionis. $\quad 4^{\circ}$. Bonnce, 1841.

ZADDACH (E. G.). Synopseos Crustaceorum Prussicorum Prodromus. $4^{\circ}$. Regionionti, 1844.

ZADDACH (E. G.). Tntersuchungen über die Entwickelung und den Bau der Gliederthiere. $\quad 4^{\circ}$. Berlin, 1854.

ZADDACH (E. G.). Beschreibung neuer oder wenig bekannter Blattwespen. $\quad 4^{\circ}$. Königsberg, 1859.

ZAERINGER (J. B.). Quædam de Historia Naturali et descriptio Salmonis farionis. 8. Friburgi, 1829.

ZAWADZKI (A.). Fauna der Galizisch-bukowinischen Wirbelthiere. 8․ Stuttgart, 1840. 
ZEDER (J. G. H.). Anleitung zur Naturgeschichte der Eingeweidewürmer. $\quad 8^{\circ}$. Hamburg, 1803.

ZEIS (E.). Beschreibung mehrerer kranker Knochen vorweltlicher Thiere.

8.. Leipzig, 1856.

ZELEBOR (J. von). See 'Novara.'

ZELLER (P. C.). Chilonidarum et Crambidarum Genera et Species. $4^{\circ}$. Berolini, 1863.

ZENKER (J. C.). Naturgeschichte schädlicher Thiere.

Text, 8․ Atlas, $4^{\circ}$. Leipzig, 1836.

ZETTERSTEDT (J. W.). Resa genom Sweriges och Norriges Lappmarker, Förrättad år 1821.

2 vols. $8^{\circ}$. Lund, 1822.

ZETTERSTEDT (J. W.). Fauna Insectorum Lapponica. Part I. $8^{\circ}$. Hammonoe, 1828.

ZETTERSTEDT (J. W.). Orthoptera Sueciæ.

8.. Lundce, 1831.

ZETTERSTEDT (J. W.). Insecta Lapponica descripta.

$4^{\circ}$. Lipsice, 1840.

ZETTERSTEDT (J. W.). Diptera Scandinaviæ deposita et descripta. 14 vols. $8^{\circ}$. Lundae, 1842-60.

ZIMMERMANN (C.). Monographie der Carabiden. 8. Berlin \& Halle, 1831.

ZIMMERMANN (E. A. G.). Specimen zoologiæ geographicæ Quadrupedum domicilia et migrationes sistens.

$4^{\circ}$. Lugduni Batavorum, 1777.

ZIMMERMANN (E. A. W.). Geographische Geschichte des Menschen. 3 vols. $8^{\circ}$. Leipzig, 1778-83.

ZINANNI (G.). See Grnanni (G.). 
Zincken (J. L. T. F.). See Periodrcals : Halle.

ZITTEL (K. A.). Beiträge zur Systematik der fossilen Spongien: $8^{\circ}$. Stuttgart, 1879.

ZOOLOGICAL RECORD (Thej. See Periodicals: London.

ZOOLOGICAL SOCIETY. See Periodicals: London.

ZOOLOGIST (The). See Pertodicals : London.

ZOOLOGISCHE GARTEN (Der). See Periodicals : Frankfort.

ZORGDRAGER (K. G.). Alte und neue Grönländische Fischerei, und Wallfischfang. $\quad 4^{\circ}$. Leipzig, 1723.

ZUNTZ (N.). See Hermany (L.). Handbuch der Physiologie. 


\section{PERIODICALS.}

\section{JOURNALS, TRANSACTIONS, AND OTHER PUBLI- CATIONS OF LEARNED SOCIETIES.}

Note.-The Periodicals are arranged alphabetically under the place of publication.

\section{ALTRINCHAM.}

The Weekly Entomologist, for 1862-63.

2 vols. in $1.8^{\circ}$. Altrincham, 1863.

\section{AMISTERDAM.}

Bijdragen tot de Dierkunde, uitgegeven door het koninklijk Zoologisch Genootschap Natura Artis Magistra.

fol. Amsterdam, 1848-69.

Nederlandsch Tijdschrift voor de Dierkunde. Von P. Bleeker, H. Schlegel en G. F. Westerinan.

Vols. I.-IV. 8․ Amsterdam, 1863-73.

\section{BATAVIA.}

Acta Societatis Scientiarum Indo-Neerlandicæ.

Vols. V., VII., \& VIII. $4^{\circ}$. Batavia, 1858-60.

\section{BERLIN.}

Archiv für Anatomie und Physiologie; herausgegeben von J. MÜLLER, \& 25 vols. $8^{\circ}$. Berlin, 1834-58. Continued at Leipzig, 1859-83. See LeIPZIG.

See also Halle: Archiv für Physiologie.

Archiv fiir Naturgeschichte. Herausgegeben ron A. F. A. Wiegmani. Vols. I.-XLIX. $8^{\circ}$. Berlin, 1835-83. 
Archiv für Zoologie und Anatomie. Herausgegeben von C. R. W. Wiedemans. 4 vols. $8^{\circ}$. Berlin, 1800-4.

Bericht uiber die Wissenschaftlichen Leistungen in der Naturgeschichte der Niederen Thiere. 1880-81.

Vol. I. $8^{\circ}$. Berlin, 1883.

Berliner Entomologische Zeitschrift.

Continued as :

$8^{\circ}$. Berlin, 1857-82.

Deutsche Entomologische Zeitschrift. $\quad 8^{\circ}$. Berlin, 1883.

Entomologische Monatsblätter. Herausgegeben von G. KrAATZ. Parts I.-III. 8º Berlin, 1876-80.

Jahresbericht der Commission zur Wissenschaftlichen Untersuchung der Deutschen Meere in Kiel.

Parts I.-IV. $4^{\circ}$. Berlin, 1873-82.

Linnæa Entomologica. Zeitschrift herausgegeben von dem Entomologischen Vereine in Stettin.

Vols. I.-XVI. 8. Berlin \& Leipzig, 1846-66.

Ornitbologisches Centralblatt. Organ für Wissenschaft und Praxis. Beiblatt zum Journal fïr Ornithologie. Herausgegeben von J. CABANis und A. ReIcHeNow.

6 vols. in $2, \&$ parts. $4^{\circ}$. Berlin, 1876-83.

Sitzungs-Bericht der Gesellschaft Naturforschender Freunde. $8^{\circ}$. Berlin, 1879-80.

Zeitschrift für Ethnologie.

Vols. I.-XV. roy. $8^{\circ}$. Berlin, 1869-83.

\section{BONN.}

Archiv für Mikroskopische Anatomie, herausgegeben von Max Schultze.

Vols. I.-XXII. \& Suppl. 8. Bonn, 1865-83.

Archiv für die gesammte Physiologie des Menschen und der Thiere. Herausgegeben von E. F. W. Pflüger.

Vols. I.-XXXI. 8․ Bonn, 1868-83. 


\section{BOSTON, U.S.}

Boston Society of Natural History.

Proceedings (incomplete). $8^{\circ}$. Boston, 1866-80. Memoirs.

$4^{\circ}$. Boston, 1871-76.

Boston Society of Natural History. Occasional Papers. Entomological Correspondence of T. W. Harris. Edited by S. H. Scudder.

8. Boston, 1869.

BRESLAU.

Beiträge zur Entomologie.

8. Brestau, 1829.

Entomologische Miscellen. Herausgegeben von dem Verein für Schlesische Insektenkunde. $\quad 8^{\circ}$. Breslau, 1874.

Zeitschrift für Entomologie, herausgegeben ron dem Verein für Schlesische Insektenkunde zui Breslau.

8. Brestau, 1847-61.

\section{BRIEG.}

Bericht des Schlesischen Tausch-Vereins für Schmetterlinge. Von Reinhold Döring.

Vols. I.-VII. $4^{\circ}$. Brieg, 1840-46.

\section{BROOKLYN, U.S.}

Bulletin of the Brooklyn Entomological Society.

Vols. I.-V. $8^{\circ}$. Brooklyn, 1878-84.

\section{BRUNSWICK.}

Archiv für Anthropologie.

Vols. I.-XVIII. $4^{\circ}$. Braunschweig, 1866-84.

\section{BRUSSELS.}

Annales de la Société Entomologique Belge.

Vols. I.-XXVI. $8^{\circ}$. Bruxelles, 1857-82.

Annales de la Société Malacologique de Belgique.

Vols. I.-XVI. $\quad 8^{\circ}$. Bruxelles, 1863, 1878, 1879-82. 
Bulletin de l'Académie Royale des Sciences et Belleslettres de Bruxelles. 8. Bruxelles, 1842.

Bulletin du Musée Royal d'Histoire Naturelle de Belgique. Vols. I. \& II. $8^{\circ}$. Bruxelles, 1882-83.

Revue Coléoptérologique publiée par C. van den BRANden. Première Année. roy. $8^{\circ}$. Bruxelles, 1882.

\section{BUENOS AYRES.}

Anales del Museo Público de Buenos-Aires, por G. Burmeister. $\quad$ Parts I.-VI. $4^{\circ}$. Buenos-Aires, 1866-74.

Periódico Zoológico. Órgano de la Sociedad Zoológica Argentina.

Vol. I. $8^{\circ}$. Buenos Aires, 1875. Vols. II.-VI. $\quad 8^{\circ}$. Córloba, 1876-80.

\section{BUFFALO.}

Bulletin of the Buffalo Society of Natural Sciences.

Vol. I. No. 1. $8^{\circ}$. Buffalo, 1873.

Vol. II. Nos. $2 \& 4$. $1874-75$.

Vol. III. Nos. 1 \& $5 . \quad 1875-77$.

Vol. IV. Nos. 1 \& $2 . \quad$ 1881-82.

The North-American Entomologist. Edited by A. R. Grote. $\quad 8^{\circ}$. Buffalo, 1879-80.

\section{CALCUTTA.}

Stray Feathers, a Journal of Ornithology for India and its Dependencies. Edited by A. Hume.

Vols. I.-XI. $\quad 8^{\circ}$. Calcutta, 1873-83.

\section{CAMBRIDGE, U.S.}

Bulletin of the Nuttall Ornithological Club.

Vols. I.-VIII. $\quad 8^{\circ}$. Cambridge, 1876-83.

\section{CANADA.}

The Canadian Entomologist.

Vols. I.-XV. 8. London, Canada, 1869-83. 


\section{CASSEL.}

Journal für Ornithologie; herausgegeben von J. CABANIs. Vols. I.-XVII. $8^{\circ}$. Cassel, 1853-69. See LeIPZIG.

Vols. XVIII.-XXXI. 80. Leipzig, 1870-83.

Malakozoologische Blätter; herausgegeben von Menke \& Pfetffer (L.). $\quad$ Vols. I.-VII. 8 ${ }^{\circ}$. Cassel, 1851-82. Vol. VIII. (Neue Folge). $\quad$ 8 . Cassel \& Berlin, 1883.

Zeitschrift für Malakozoologie; herausgegeben von Menke \& Pfatffer (L.). $\quad 8^{\circ}$. Hannover \& Cassel, 18+8-53.

\section{COLONBO.}

Journal of the Ceylon Branch of the Royal Asiatic Society. $8^{\circ}$. Colombo, 1850-58.

\section{COPENHAGEN.}

Naturhistorisk Tidsskrift stiftet af H. KRUYER, udgivet af J. C. Sсніӧрте. Vols. I.-VI. 8. Kjöbenhavn, 1861-70.

Nordisk Tidsskrift for Fiskeri.

Vols. I.-VII. $8^{\circ}$. Copenhagen, 1874-82.

Videnskabelige Meddelelser fra den naturhistoriske Forening i Kjöbenharn.

$8^{\circ}$. Copenhagen, 1854.

\section{CORDOVA.}

Periódico Zoológicu. See Buenos Arres.

\section{DRESDEN.}

Mittheilungen aus dem königl. Zoologischen Museum zu Dresden herausgegeben von A. B. MEYer.

Parts I.-III. $4^{\circ}$. Dresden, 1877-8.

\section{DUBLIN.}

Reports of the Royal Zoological Society of Ireland.

8. Dublin, $1862-76$. 


\section{EDINBURGH.}

Annals of Anatomy and Physiology, conducted by J. Goopsir. Parts I.-III. (all published). $8^{\circ}$. Edinburgh \& London, $1850-53$.

The Edinburgh Journal of Natural History and of the Physical Sciences, conducted by W. MacGinimray.

2 vols. in 1. fol. Edinburgh, 1835-40.

\section{FLORENCE.}

Bollettino della Società Entomologica Italiana.

Vols. I. XV. $8^{\circ}$. Firenze, 1869-83.

\section{FRANKFORT.}

Abhandlungen der Senckenbergischen naturforschenden Gesellschaft in Frankfurt a. Main. Vol. XII. Nos. 2, 3, 6, 8, 9, 10, 11. Vol. XIII. Nos. 1, 2.

4. Frankfurt am Main, 1880-83.

Jahrbücher der Deutschen Malako-zoologischen Gesellschaft, redigirt von Dr. W. KoвeLt.

8. Frankfurt am Main, 1880-84.

Der Zoologische Garten. Organ der Zoologischen Gesellschaft in Frankfurt a. Main; herausgegeben von W. F. Weinland, C. Brtch, Dr. Stiebex und F. C. Noll.

8. Frankfurt am Main, 186t-83.

\section{GENEVA.}

Bulletin de la Société Ornithologique Suisse.

Vols. I. \& II. 8. G'enève \& Bate, 1865-70.

\section{GENOA.}

Archivio per la Zoologia, l' Anatomia e la Fisiologia.

See Modena.

Vols. I. \& II. $8^{\circ}$. Genova, 1862-63. 


\section{GHENT.}

Archives de Biologie publiées par E. van Beneden et C. van BAMBEKE.

Vols. I.-III., vol. IV. part 1. $8^{\circ}$. Gand \& Leipzig, 1883.

\section{GÖTEBORG.}

Göteborgs och Bohus häns Hushållnings-sällskaps Quartalsskrift. Bihang. Nya Handlingar rörande Sillfisket i Bohus-länska Skärgården.

8․ Göteborg, 1874.

\section{THE HAGUE.}

Tijdschrift voor Entomologie, onder redactie T. vAN DER Hoeven, \&c.

Vols. I.-XXVI. 8'. 'S Gravenhage, 1858-83.

HALLE.

Archiv für Physiologie. Herausgegeben von J. C. REIL.

Continued as:

8. Halle, 1796-1815.

Deutsches Archiv für Physiologie. Von J. F. МескеL.

$8^{\circ}$. Halle \& Berlin, 1815-19. Halle, 1820-23. Continued as :

Archiv für Anatomie und Physiologie. Von J. F. MEcket.

8. Leipzig, 1826-30.

Continued by MüLler, 1834 \&c. See BerLIN.

Magazin der Entomologie, herausgegeben von E. F. GERMaR und J. L. T. F. Zincken, genannt SoMner.

4 vols. $8^{\circ} .1813-21$.

HAMBURG.

Journal des Museum Godeffroy.

Hefte I.-XIV. $4^{\circ} . \quad$ Hamburg, 1873-79.

See Museum (Godefrroy).

HANOVER.

Zeitschrift für Malakozoologie. See Cassed. 


\section{KIEL.}

Zoologisches Magazin. Ton C. R. W. Wiedemary.

Parts I. \& II. $8^{\circ}$. Kiel, 1817-23.

\section{LEIDEN.}

Abhandlungen aus dem Gebiete der Zoologie und vergleichenden Anatomie. Ton H. Schleger.

Parts I. \& II. $4^{\circ}$. Leiden, 1841-43.

Niederländisches Archir für Zoologie, herausgegeben von E. Selenka \& C. K. Hoffuary. ' $8^{\circ}$. Leiden, 1871-81.

\section{LEIPZIG.}

Archir für Anatomie, Physiologie und wissenschaftliche Medicin, herausgegeben von J. MüLLER, \&c.

Seo Berlin.

Tols. I.-XXXII. So. Leipzig, 1859-83.

Archir für Anatomie und Physiologie.

See HaLIF.

Archir für Anatomie und Physiologie, herausgegeben von W. His, W. Braune \& E. Du Bois-Reymond.

Fols. I.-X. $8^{\circ}$. Leipzig, 1877-84.

Journal für Ornithologie, herausgegeben von J. CABAxIs.

See Casser.

Linnea Entomologica. See BerLiv.

Morphologisches Jahrbuch, herausgegeben von C. GEGENBACR. Vols. I.-IX. 80. Leipzig, 1875-83.

Untersucliungen ans dem Institute für Physiologie und Histologie in Graz, herausgegeben ron A. RoLLETt.

Parts I.-II. $8^{\circ}$. Leipzig, 1870-71.

Zeitschrift für Entomologie, herausgegeben von E. F. Germar. Vols. I.-V. 8*. Leipzig, 1839-44. 
Zeitschrift für wissenschaftliche Zoologie, herausgegeben von C. T. จ. Stebold \& A. Kör.tiker.

\section{Vols. I.-XXXVI. 8. Leizpig, 1849-83.}

Zoologischer Anzeiger herausgegeben von J. V. CARUs.

Vols. I.-VI. $8^{\circ}$. Leipzig, 1878-83.

\section{LONDON.}

Annals and Magazine of Natural History.

1st series. Vols. I.-XX.

$8^{\circ}$. London, 1838-83.

2nd series. Vols. I.-XX.

$1838-47$.

3rd series. Vols. I. $-\mathrm{XX}$.

$1848-57$.

4th series. Vols. I.-XX.

$1858-67$.

5th series. Vols. I.-VI.

$1868-77$.

1878-83.

Anthropologia.

Part I.-V. and Supplement. 1 vol. $8^{\circ} . \quad 1873-76$.

Anthropological Review.

Vols. I.-VIII. 8. London, 1863-70.

Catalogue of Scientific Papers compiled and published by the Royal Society of London. 8 vols. $4^{\circ}$. London, 1867-79.

Catalogue of the Library of the Royal Geographical Society.

$8^{\circ}$. London, 1865.

Supplement. $8^{\circ}$. London, 1871. Second Supplement. $8^{\circ}$. London, 1880.

Catalogue of the Map Room of the Royal Geographical Society, 1881.

$8^{\circ}$. London, 1882.

Catalogue of the Natural History Library of the Linnean Society of London. Parts 1-3. $\quad 8^{\circ}$. London, 1866-77.

Catalogue of the Library of the Zoological Society of London.

8. London, 1880.

Supplement. $\quad 8^{\circ}$. London, 1883.

Cistula Entomologica, sire Insectorum novorum diagnoses. Vols. I.-III. 80. London, 1869-83. 
Entomological Magazine.

Vols. I.-V. $8^{\circ}$. London, 1832-37.

Entomologist's Monthly Magazine.

Vols. I.-XX. $8^{\circ}$. London, 1864-83.

The Entomologist. Edited by E. NEwMan and J. T. Carrington. $\quad$ Vols. I.-XVI. $8^{\circ}$. London, 1840-83.

The Entomologist's Annual. Edited by H. T. Stainton.

8. London, 1855-74.

The Entomologist's Weekly Intelligencer.

10 vols. in 5. $8^{\circ}$. London, 1856-61.

The Field : or Country Gentleman's Newspaper.

Vols. I.-LX. fol. London, 1853-82.

The Ibis : a Magazine of General Ornithology.

1st series, edited by P. L. Sclater.

8. London, 1859-83.

Vols: I.-VI. 1859-64.

2nd series, edited by A. Newton.

Vols. VII.-XII. 1865-70.

3rd series, edited by P. L. Sclater \& O. Salvin.

Vols. XIII.-XVIII. 1871-76.

4th series, edited by P. L. Sclater \& O. Salvin.

Vols. XIX.-XXIV. 1877-82.

5th series, edited by P. L. Sclater \& H. Saunders.

Vols. XXV. to date. 1883.

The Journal of Anatomy and Physiology.

Vols. I.-XVII. and parts. $8^{\circ}$. London, 1867-83.

Journal of Anthropology.

Continued as:

Vol. I., nos. 1-3. $8^{\circ}$. 1870-71.

The Journal of the Anthropological Institute.

Vols. II.-XII. $8^{\circ}$. London, 1873-83.

The Journal of the Anthropological Institute of Great Britain and Ireland.

Vol. I.-XII. $8^{\circ}$. London, 1872-83. 
Journal of the Anthropological Society.

Vols. II.-VIII. $8^{\circ}$. London, 1864-83.

Journal of Entomology, descriptive and geographical.

8. London, 1862-66.

Journal of the Proceedings of the Entomological Society.

8. London, 1841.

Journal of the Proceedings of the Linnean Society. Zoology. Vols. I.-XVI. 8 . London, 1857-83.

Magazine of Natural History, and Journal of Zoology, Botany, \&c. By J. C. Lovdon. 8 vols. 8․ 1829-35. New Series by E. Charlesworth. 80. London, 1837-40.

Magazine of Zoology and Botany. Conducted by Sir W. JaRdine, Bart., P. J. Selby, \& G. Johnston.

2 vols. $8^{\circ}$. London, 1837-38.

Nature : a weekly Illustrated Journal of Science.

$4^{\circ}$. London, 1870-83.

Popular Magazine of Anthropology.

Vol. I. $8^{\circ}$. London, 1866.

Quarterly Journal of Conchology.

Continued as :

Journal of Conchology. Vols. I.-IV. $8^{\circ}$. 1874-83.

The Substitute, or Entomological Exchange Facilitator.

Vol. I. (all published). $8^{\circ}$. London, 1856-57.

Transactions of the Entomological Society.

$8^{\circ}$. London, 1807.

Continuation. $8^{\circ}$. London, 1834-83.

Transactions of the Geological Society of London.

$4^{\circ}$. London, 1832-45. 
Willughby Society. Reprints :

ACDouIn (J. V.). Explication sommaire des planches d'Oiseaux de l'Égypte et de la Syrie, publiées par J.-C. Savigny [1828]. $\quad 8^{\circ}$. London, 1883.

Bartox (B. S.). Fragments of the Natural History of Pennsylvania [1799]. $\quad 4^{\circ}$. London, 1883.

Desfoxtaines (R. L.). Mémoire sur quelques nouvelles espèces d'Oiseaux des Côtes de Barbario [1789]. 4. London, 1880.

Forster (J. R.). Animals of Hudson's Bay [1772].

$8^{\circ}$. London, 1882.

Forster (J. R.). Catalogue of the Animals of North America, or Faunula Americana [1771].

8. London, 1882.

Leach (W. E.). Systematic Catalogue of the specimens of the indigenous Mammalia and Birds that are preserred in the British Museum: with their localities and authorities [1816].

8. London, 1882.

Lichtenstein (A. A. H.). Catalogus Rerum naturalium rarissimarum [1793]. $\quad 8^{\circ}$. London, 1882.

Scopoli (G. A.). Ornithological Papers from his Deliciae Florae et Faunae Insubricae [1786-88].

4. London, 1882.

Sмiтн (Sir A.). Miscellaneous Ornithological Papers [1830-33]. $\quad 8^{\circ}$. London, 1880.

Toxstall (M.). Ornithologia Britannica [1771].

8. London, 1880.

The Zoological Record. Edited by A. Günther, A. Newtox, \& E. C. Rire. $\quad 8^{\circ}$. London, 1865-80.

1st series, edited by A. Günther. Tols. I.-VI. $1865-70$.

2nd series, edited by A. Newtox. Tuls. VII.-XII. $1871-76$.

3rd series, edited by E. C. Rre. Tols. XIII.-XVIII. $1877-82$.

4th series, edited by E. C. RYE. Vols. XIX. to date. 1883. 
Zoological Society of London.

Proceedings of the Committee.

$8^{\circ}$. London, 1830-32.

Proceedings of the Zoological Society.

$8^{\circ}$. London, 1833-82.

Transactions of the Zoological Society.

Vols. I.-X. $4^{\circ}$. London, 1835-83.

Zoological Journal. Vols. I.-V. $8^{\circ}$. London, 1825-35.

The Zoologist : a Monthly Magazine of Natural History.

1st series, edited by E. Newrax. Vols. I.-XXIII.

1843-65.

2nd series, edited by E. Newran. Vols. XXIV.XXXIV.

1866-76.

3rd series, edited by J. E. Hartive. Vols. XXXV.XII.

$1877-83$.

LONDON, ONTARIO.

The Canadian Entomologist.

Vols. I.-XV. $\quad 8^{\circ}$. London, 1869-83.

\section{LUND.}

Lunds Universitets Års-skrift.

Vol. IX. $4^{\circ}$. Lund, 1872.

Opuscula Entomologica. Ed. C. G. Thomson.

Fasc. I.-VIII. $8^{\circ}$. Lund, 1869-77.

\section{MILAN.}

Bollettino Scientifico, redatto dai G. AchiLLe, M. LEopordo e Z. Giovanus.

Anno I.-V. $8^{\circ}$. Milano \& Pavia, 1872-83.

\section{MODENA.}

Archivio per la Zoologia, l' Anatomia e la Fisiologia.

Per Canestrini, G. Doria, P. M. Ferrari, e M. Lessona.

Vols. I. \& II., in 1 vol. $8^{\circ}$. Genova, 1862-63.

Vols. III. \& IV., in 1 vol. $8^{\circ}$. Moclena, 186t-66. 
MONTROSE.

Reports of the Directors of the Natural-History Snciety of Montrose.

$8^{\circ}$. Montrose, 1860-73.

\section{MOSCOW.}

Bulletin de la Société Impériale des Naturalistes de Moscou. 8. Moscou, 1829-84.

\section{MUNICH.}

Mittheilungen des Münchener Entomologischen Vereins. Redac. V. HARoLD.

Vols. I.-IV. (8 pts.) $8^{\circ}$. München, 1877-80. [All published.]

\section{NAPLES.}

Annuario del Mruseo Zoologico della R. Università di Napoli. Anno I. \& IV. 8'. Napoti, 1862, 1867.

\section{NEIV YORK.}

Annals of the Lyceum of Natural History of New York.

$$
8 \text { rols. } 8^{\circ} \text {. New York, 1824-67. }
$$

Papilio: the organ of the New York Entomological Club. Edited by H. Enwards. Tols. I.-III. $8^{\circ}$. 1881-83.

Transactions of the Linnean Society of New York.

$$
\text { Vol. I. } 8^{\circ} .1882 .
$$

\section{PARIS.}

Annales de l'Institut Agronomique. $\quad 4^{\circ}$. Paris, 1852.

Annales de la Société Entomologique de France.

1st series. Vols. I.-XI.

$8^{\circ}$. Puris, $1832-83$.

2nd series. Vols. I. $-X$.

3rd series. Vols. I.-VIII.

4th series. Tols. I.-X. \& Suppl.

5th series. Tols. I.-X. \& Suppl.

$1832-42$.

$1843-52$.

1853-60.

6th series. Vols. I. \& II.

1861-70.

Tables Générales. 
Annales de la Société Malacologique de Belgique.

Tomes 1-13. $8^{\circ}$. Paris, 1863-78.

Annales des Sciences naturelles. Rédigées par J. V. Audouin et H. Mrlne-Edwards. Zoologie.

2nd series. Vols. I.-XX.

$8^{\circ}$. Paris, $1834-83$.

3rd series. Vols. I. $-\mathrm{XX}$.

1834-43.

4th series. Vols. I. $-\mathrm{XX}$.

$1844-53$.

5th series. Vols. I.-XX.

1854-63.

6th series. Vols. I. $-\mathrm{XV}$.

1864-73.

1874-83.

Bulletin de la Société d'Anthropologie de Paris.

1st series. Vols. I.-VI. and General Index. 1860-65.

2nd series. Vols. I.-XII.

3rd series. Vols. I.-VI.

$1878-83$.

Bulletin de la Société Zoologique de France.

Vols. I.-VIII. $8^{\circ}$. Paris, 1876-83.

Bulletin Mensuel de la Société d'Acclimatation.

1st series. Vols. I. -X.

8. Paris, $1855-83$.

2nd series, Vols. I. $-\mathrm{X}$.

$1855-63$.

3rd series. Vols. I.-X.

$1864-73$.

$1874-83$.

Journal de l'Anatomie et de la Physiologie. Publié par C. Robin.

8. Paris, 1864-77.

Journal de Conchyliologie, comprenant l'Étude des Animaux, des Coquilles vivantes et des Coquilles fossiles.

Vols. I.-XXXIV. $8^{\circ}$. Paris, 1850-83.

Journal de Zoologie, comprenant les différentes branches de cette science, par P. Gervais. $\quad 8^{\circ}$. Paris, 1872-77.

L'Abeille, Mémoires d'Entomologie.

Continued as :

L'Abeille, Journal d'Entomologie.

16 vols. $12^{\circ}$. Paris, $1864-7 \overline{8}$.

Magasin de Zoologie.

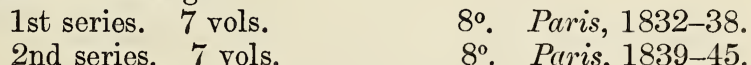


Mémoires de la Société d'Anthropologie de Paris.

1st series. Vols. I.-III.

2nd series. Vols. I. \& II.

Revue de Sériculture Comparée.

Vols. I.-IV. $8^{\circ}$. Paris, 1863-66.

Revue et Magasin de Zoologie.

Vols. I.-XXX. 80. Paris, 1849-78.

Revue Zoologique. Vols. I.-X. 8o. Paris, 1838-48.

PHILADELPHIA.

American Journal of Conchology.

Vols. I.-VII. $8^{\circ}, \quad 1865-72$.

Journal of the Academy of Natural Sciences of Philadelphia. Vols. I.-XYIII. 8. Philadelphia, 1817-34.

Proceedings of the Academy of Natural Sciences of Philadelphia. Vols. I.-XLI. 80. Phitadelphia, 1843-83.

Proceedings of the Entomological Society of Philadelphia.

Vols. I.-VII. 8. Philadelphia, 1863-69.

Transactions of the American Entomological Society.

Vols. I.-X. $8^{\circ}$. Philadelphia, 1867-83.

PISA.

Bollettino Malacologico Italiano.

Vols. I.-IV. $8^{\circ}$. 1868-71. Vols. V.-VIJ. (one part each). 1872-74.

\section{RATISBON.}

Correspondenzblatt für Sammler von Insecten, insbesondere von Schmetterlingen. Vol. I. 8 . Regenshurg, 1860. 


\section{RIPON.}

The Naturalist Trout-Fisher.

No. 1, July 21st, 1882. $4^{\circ}$. 1882.

\section{$\mathrm{S}^{\mathrm{T}}$ PETERSBURG.}

Horæ Societatis Entomologicæ Rossicæ.

Vols. I. XV. $4^{\circ}$. St. Petersburg, 1861-83.

Mélanges Biologiques.

Tomes I.-XI. $\quad 8^{\circ}$. St. Petersburg, 1849-82.

Trudui Russkago Entomologicheskago Obshchestva.

(Transactions of the Russian Entomological Society.)

Vols. I.-XII. $8^{\circ}$. St. Petersburg, 1861-81.

Vol. XIII. 1882.

\section{SALZBURG.}

Jahres-Bericht des vaterländischen Mruseums CarolinoAugusteum des Landes-Hauptstadt Salzburg. $\quad 8^{\circ} .1859$.

\section{SAN FRANCISCO.}

Proceedings of the California Academy of Natural Sciences. Vol. III. parts 1 \& 2. 8 ${ }^{\circ}$. San Francisco, 1863-64.

\section{SCHAFFHAUSEN.}

Nittheilungen der Schweizerischen Entomologischen Gesellschaft. Vols. I.-VI. $8^{\circ}$. Schafthausen, 1862-82.

\section{STETTIN.}

Entomologische Zeitung. 8.. Stettin, 1840-82.

Zeitschrift des Verbandes der Ornithologischen Vereine Pommerns und Mecklenburgs.

Nos. 1-6. $8^{\circ}$. Stettin, 188\%.

Zeitschrift für Ornithologie.

2 Jahr. No. 8. August 1883. 


\section{STOCKHOLM.}

Biologische Untersuchungen herausgegeben von G. RETzIUS.

Vols. I. \& II. Stockholm, 1881-82.

Entomologisk Tidskrift.

Vols. I.-IV. $\quad 8^{\circ}$. Stockholm, 1880-83.

\section{SYDNEY.}

Transactions of the Entomological Society of New South Wales. $\quad$ Vol. I. parts 2 \& 5. 80. Sydney, 1864-66. Vol. II. part 1. $\quad 8^{\circ}$. Sydney, 1869.

TASMANIA.

Papers and Proceedings of the Royal Socicty of Van Diemen's Land. Vol. I. part 3. $\quad 8^{\circ}$. Tasmania, 1851.

\section{TROMSÖ.}

Tromsö Museums Aarshefter.

Parts I. \& II. 8. Tromsö, 1878-79.

\section{TRURO.}

Reports of the Royal Institution of Cornwall.

$$
8^{\circ} \text {. Truro, 1839-72. }
$$

\section{TURIN.}

Studi Entomologici pubblicati per cura di T. BAUDI e di E. Truqui.

8. Torino, 1848.

\section{VIENNA.}

Arbeiten aus dem Zoologischen Institute der Universität Wien und der Zoologischen Station in Triest. Herausgegeben von C. Clats. I.-IV. $8^{\circ}$. Wien, 1878-82.

Festschrift der Zoologisch-botanischen Gesellschaft. $4^{\circ}$. Wien, 1876.

Nittheilungen der Anthropologischen Gesellschaft in Wien.

Vols. I.-X. $\quad 8^{\circ}$. Wien, 1871-81.

Vols. XI.-XIII. $4^{\circ}$. Wien, 1882-83. 
Mittheilungen des Ornithologischen Vereines in Wien.

Vols. I.-VI. $4^{\circ}$. Wien, 1877-82.

Wiener Entomologische Monatschrift. Verantwortliche Redacture J. Lederer und L. MiLLer.

Vols. I.-VIII. $8^{\circ}$. Wien, 1857-64.

Wiener Entomologische Zeitung.

Vols. I. \& II. $8^{\circ}$. Wien, 1882-83.

\section{WASHINGTON, U.S.}

Bulletin of the United States National Museum.

No. 1. Check-list of North American Batrachia and Reptilia, by E. D. Cope. $\quad 8^{\circ}$. Washington, 1875.

No. 7. Insects injurious to Forest and Shade Trees. By A. S. Packard. 8. Washington, 1881.

No. 16. Synopsis of the Fishes of North America. By D. S. Jorda and C. H. Gilbert. $\quad 8^{\circ} .1883$.

WELLINGTON, N.Z.

Transactions and Proceedings of the New-Zealand Institute. Vols. I.-V. $8^{\circ}$. Wellington, 1869-73.

\section{WÜRZBURG.}

Arbeiten aus dem Zoologisch-Zootomischen Institute. Herausgegeben von C. Semper.

Vols. I.-X. $8^{\circ}$. Würzburg, 1872-81. 






